Florida International University FIU Digital Commons

$11-9-2011$

\title{
Theoretical Study of Chloroperoxidase Catalyzed Chlorination of beta-Cyclopentanedione and Role of Water in the Chlorination Mechanism
}

Cassian D'Cunha

Florida International University, cassian.dcunha@gmail.com

DOI: $10.25148 /$ etd.FI11120708

Follow this and additional works at: https://digitalcommons.fiu.edu/etd

\section{Recommended Citation}

D'Cunha, Cassian, "Theoretical Study of Chloroperoxidase Catalyzed Chlorination of beta-Cyclopentanedione and Role of Water in the Chlorination Mechanism" (2011). FIU Electronic Theses and Dissertations. 515.

https://digitalcommons.fiu.edu/etd/515 


\section{FLORIDA INTERNATIONAL UNIVERSITY}

Miami, Florida

THEORETICAL STUDY OF CHLOROPEROXIDASE CATALYZED

CHLORINATION OF BETA-CYCLOPENTANEDIONE AND ROLE OF WATER IN

THE CHLORINATION MECHANISM

A dissertation submitted in partial fulfillment of the

requirements for the degree of

DOCTOR OF PHILOSOPHY

in

CHEMISTRY

by

Cassian D’Cunha 
To: Dean Kenneth Furton

College of Arts and Sciences

This dissertation, written by Cassian D'Cunha, and entitled Theoretical Study of Chloroperoxidase Catalyzed Chlorination of beta-Cyclopentanedione and Role of Water in the Chlorination Mechanism, having been approved in respect to style and intellectual content, is referred to you for judgment.

We have read this dissertation and recommend that it be approved.

$\begin{array}{r}\hline \text { Alexander Mebel } \\ \hline \text { Xiaotang Wang } \\ \hline \text { Bernard Gerstman } \\ \hline \text { Kenneth Furton } \\ \hline \text { David Chatfield, Major Professor }\end{array}$

Date of Defense: November 9, 2011

The dissertation of Cassian D'Cunha is approved.

Dean Kenneth Furton

College of Arts and Sciences

Dean Lakshmi N. Reddi

University Graduate School

Florida International University, 2011 
(C) Copyright 2011 by Cassian D'Cunha

All rights reserved. 


\section{DEDICATION}

I dedicate this dissertation to my parents. To my mother, my role model, for her constant love and support, and in loving memory of my father. 


\section{ACKNOWLEDGMENTS}

I would first like to thank my committee members. Their help, encouragement and patience during the various stages of my dissertation have been most appreciated. I would like to thank Dr. Xiaotang Wang for being particularly helpful during the design phase of my dissertation. I would like to thank Dean Kenneth Furton for taking time from his extremely busy schedule to serve on my committee. I would like to thank Dr. Bernard Gerstman for taking an interest in my research and inviting me to present my research to his research group on several occasions. I would like to thank Dr. Alexander Mebel for being supportive and patient, and for answering the many questions I had regarding quantum mechanics approaches. I would especially like to thank Dr. David Chatfield, my major professor. He has been very supportive, encouraging and patient, and I sincerely appreciate the time I spent under his guidance. I cannot thank him enough for the time he took to discuss research, guide me through my dissertation and make resources available for my research. His mentorship was invaluable and much appreciated.

My experience in the graduate program in Department of Chemistry \& Biochemistry at Florida International University has been very rewarding. The professors in the department have been very approachable, accommodating and always willing to help. I would also like to thank my peers in Dr. Mebel's and Dr. Chatfield's research groups. The invaluable discussions during our group meetings and the discussions in the research laboratory have been invaluable and enriched my learning experience.

I would like to thank my friends and roommates over the years. They have been my family away from home while I was pursuing my degree. I would also like to thank the Martinez family that has all but adopted me and made me feel welcome in their home. 


\begin{abstract}
OF THE DISSERTATION
THEORETICAL STUDY OF CHLOROPEROXIDASE CATALYZED

CHLORINATION OF BETA-CYCLOPENTANEDIONE AND ROLE OF WATER IN

THE CHLORINATION MECHANISM
\end{abstract}

by

\title{
Cassian D'Cunha
}

Florida International University, 2011

Miami, Florida

Professor David Chatfield, Major Professor

Chloroperoxidase (CPO) is a potential biocatalyst for use in asymmetric synthesis. The mechanisms of CPO catalysis are therefore of interest. The halogenation reaction, one of several chemical reactions that $\mathrm{CPO}$ catalyzes, is not fully understood and is the subject of this dissertation.

The mechanism by which CPO catalyzes halogenation is disputed. It has been postulated that halogenation of substrates occurs at the active site. Alternatively, it has been proposed that hypochlorous acid, produced at the active site via oxidation of chloride, is released prior to reaction, so that halogenation occurs in solution. The freesolution mechanism is supported by the observation that halogenation of most substrates often occurs non-stereospecifically. On the other hand, the enzyme-bound mechanism is supported by the observation that some large substrates undergo halogenation stereospecifically.

The major purpose of this research is to compare chlorination of the substrate $\beta$ cyclopentanedione in the two environments. One study was of the reaction with limited 
hydration because such a level of hydration is typical of the active site. For this work, a purely quantum mechanical approach was used. To model the aqueous environment, the limited hydration environment approach is not appropriate. Instead, reaction precursor conformations were obtained from a solvated molecular dynamics simulation, and reaction of potentially reactive molecular encounters was modeled with a hybrid quantum mechanical/molecular mechanical approach. Extensive work developing parameters for small molecules was pre-requisite for the molecular dynamics simulation.

It is observed that a limited and optimized (active-site-like) hydration environment leads to a lower energetic barrier than the fully solvated model representative of the aqueous environment at room temperature, suggesting that the stable water network near the active site is likely to facilitate the chlorination mechanism.

The influence of the solvent environment on the reaction barrier is critical. It is observed that stabilization of the catalytic water by other solvent molecules lowers the barrier for keto-enol tautomerization. Placement of water molecules is more important than the number of water molecules in such studies. The fully-solvated model demonstrates that reaction proceeds when the instantaneous dynamical water environment is close to optimal for stabilizing the transition state. 


\section{TABLE OF CONTENTS}

CHAPTER

PAGE

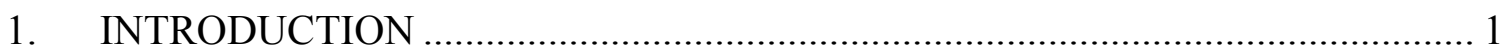

1.1. Chloroperoxidase ………………………….................................... 1

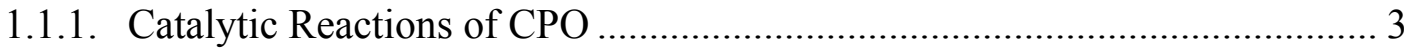

1.1.2. Previous Work on Mechanism of CPO-catalyzed Halogenation Reaction ... 6

1.1.3. Substrate Used to Study Reaction.............................................................. 8

1.2. Chlorination Mechanism of CPO.............................................................. 9

1.3. Previous theoretical studies of keto-enol tautomerization .............................. 13

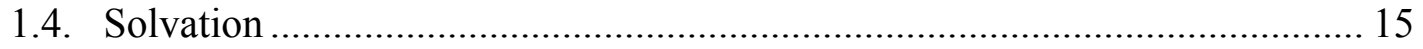

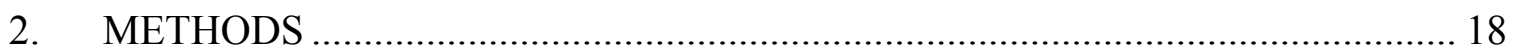

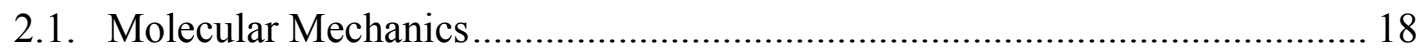

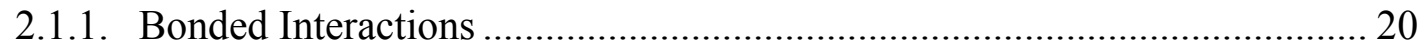

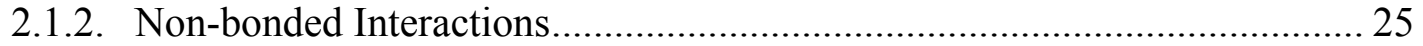

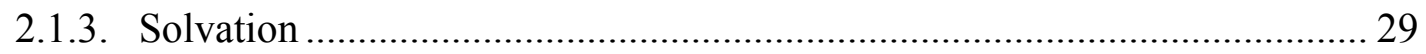

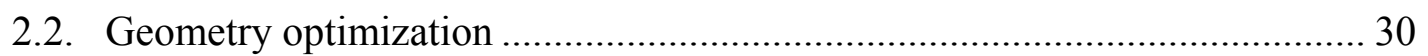

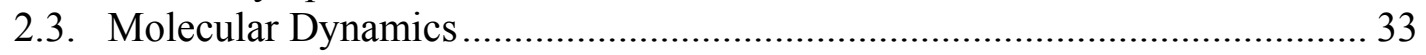

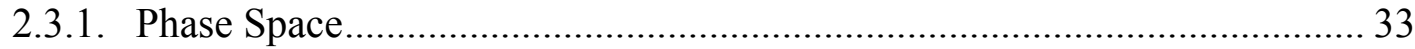

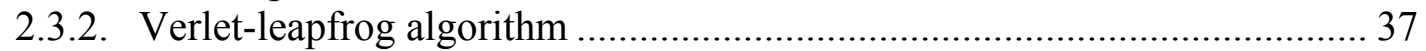

2.3.3. Periodic Boundary Conditions .................................................................. 41

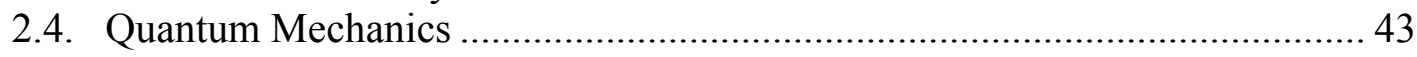

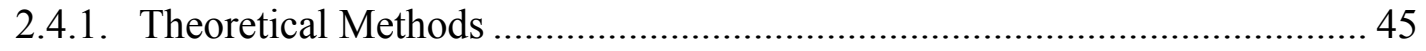

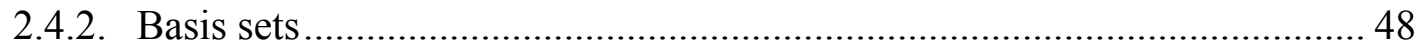

2.5. Quantum Mechanics / Molecular Mechanics …………................................... 51

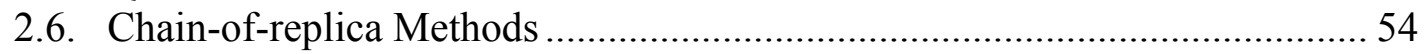

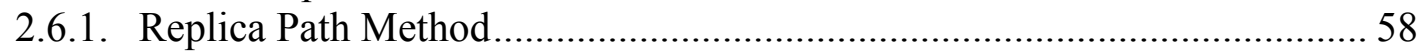

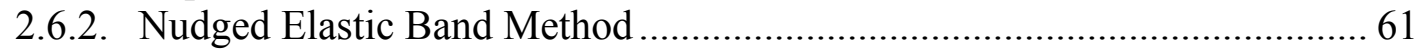

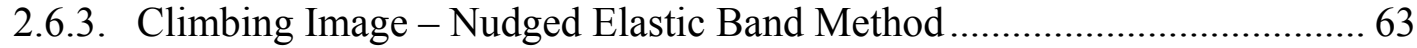

2.6.4. Adaptive Nudged Elastic Band Method ....................................................... 64

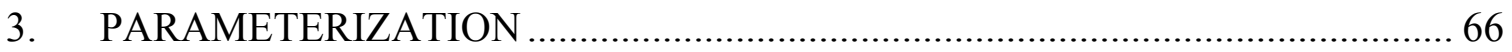

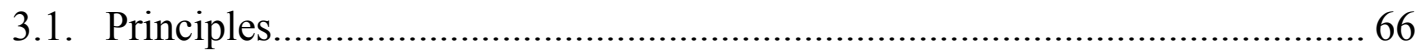

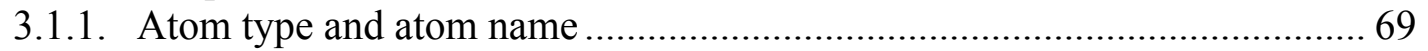

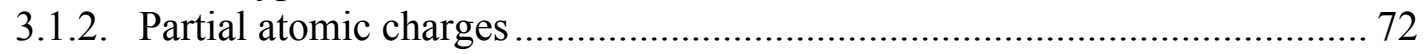

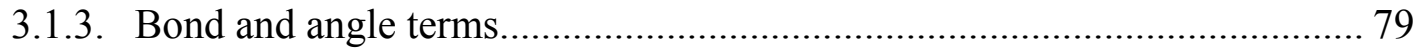

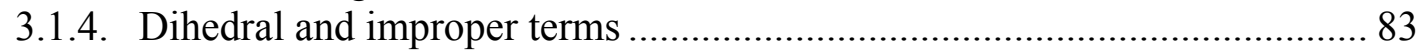

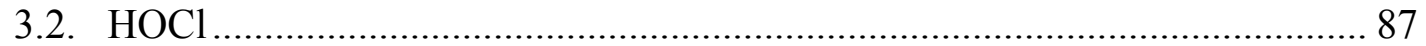

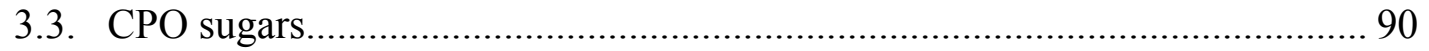

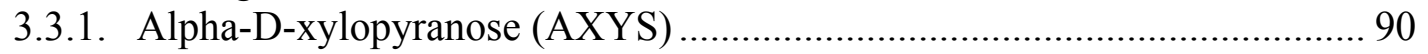

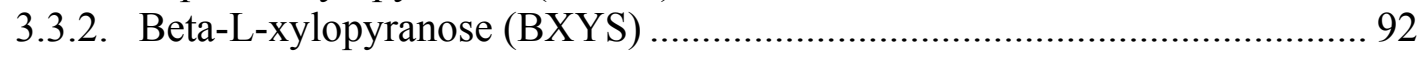

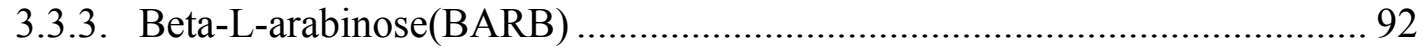

3.3.4. N-Acetyl-Glucosamine (BNAG) ……………........................................ 93 


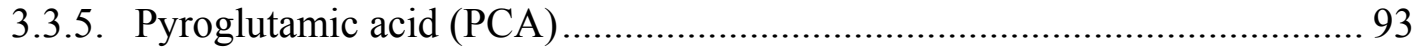

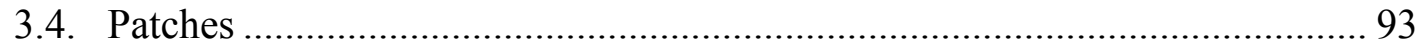

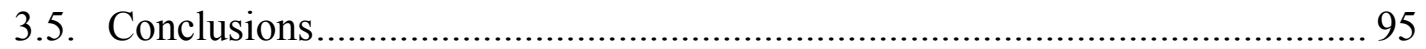

4. STUDY OF CPD DIKETO $\rightarrow$ KETO-ENOL TAUTOMERIZATION WITH

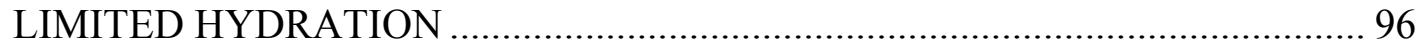

4.1. Gas phase / Intramolecular proton transfer ..................................................... 96

4.2. Intermolecular proton transfer with one catalytic water molecule ................. 96

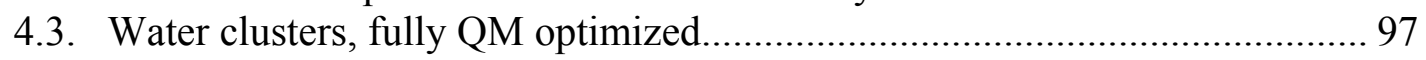

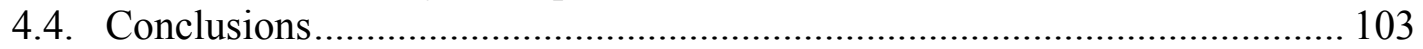

5. STUDY OF CPD DIKETO $\rightarrow$ KETO-ENOL TAUTOMERIZATION UNDER FULL SOLVATION CONDITIONS ......................................................... 104

5.1. MD simulation and selection of frames ................................................... 104

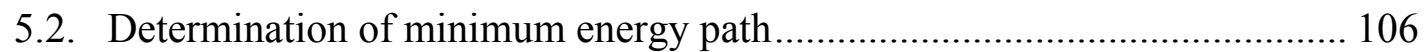

5.2.1. Determining MEP using Replica Path Method........................................ 107

5.2.2. Locating transition state using Climbing Image Nudged Elastic Band .... 112

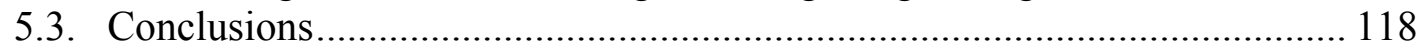

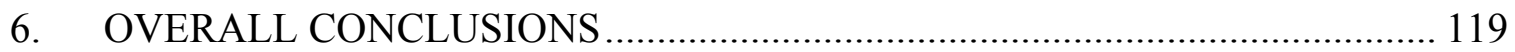

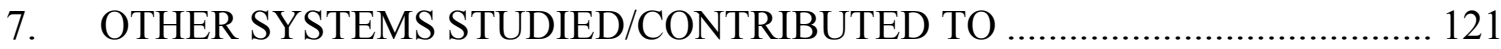

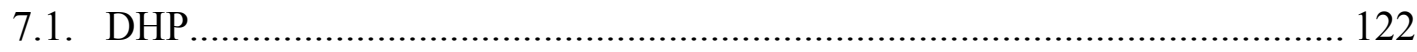

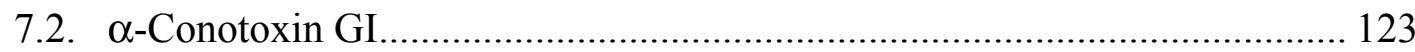

7.3. Chloroperoxidase epoxidation ................................................................ 133

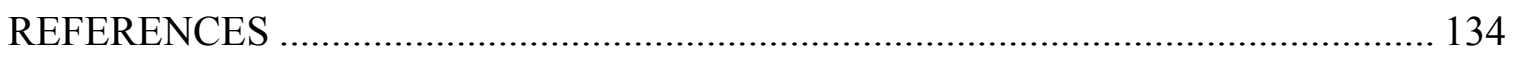

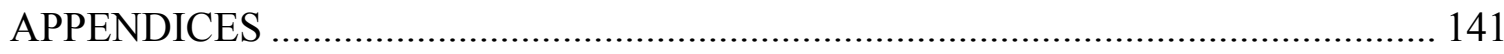

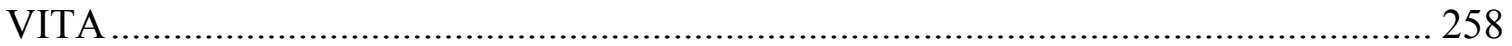




\section{LIST OF TABLES}

TABLE

PAGE

Table 1: CPD Atom Names and Atom Types............................................................... 72

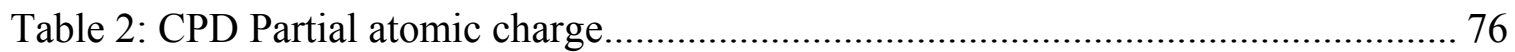

Table 3: $\mathrm{CPD}-\mathrm{H}_{2} \mathrm{O}$ Interaction Energies .................................................................... 77

Table 4: $\mathrm{CPD}-\mathrm{H}_{2} \mathrm{O}$ Interaction Distances................................................................. 78

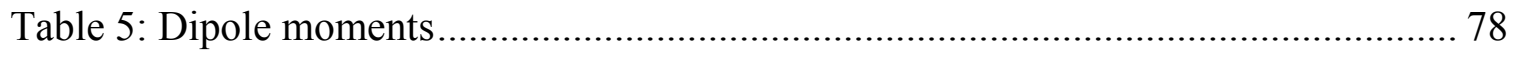

Table 6: QM calculated Frequencies $\left(\mathrm{cm}^{-1}\right)$ and $\%$ contribution...................................... 81

Table 7: MM calculated Frequencies $\left(\mathrm{cm}^{-1}\right)$ and $\%$ contribution .................................... 82

Table 8: CHARMM Topology File for CPD ................................................................ 85

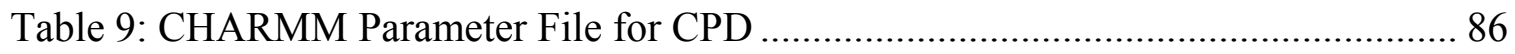

Table 10: HOCl Atom Names and Atom Types ........................................................ 88

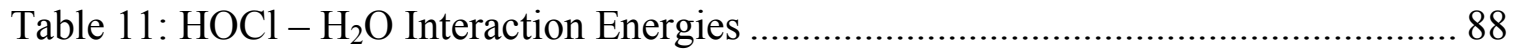

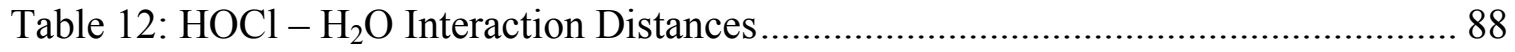

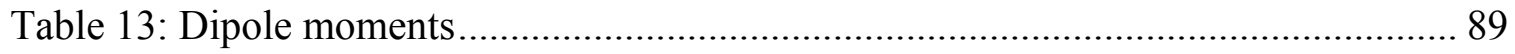

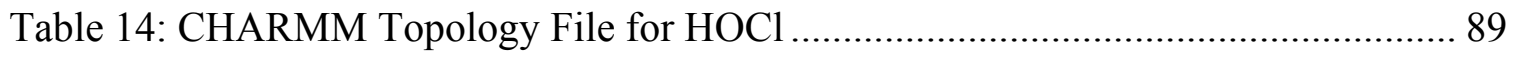

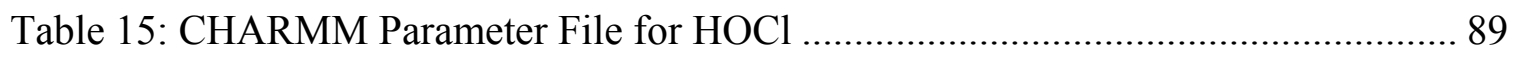

Table 16: Patch to connect two mannose sugar molecules............................................... 94

Table 17: Reaction energies and barrier heights $(\mathrm{kcal} / \mathrm{mol})$ for reaction in water clusters

Table 18: Frame 1: Normalized energy level for each replica for every 100 ABNR optimization cycles. The maximum energy for each optimization cycle is highlighted

Table 19: Frame 1: Average RMS Gradient for every 100 ABNR optimization steps .. 110

Table 20: Frame 1: RMSD and \% RMSD deviation from neighboring replicas. 111 
Table 21: Barrier heights $(\mathrm{kcal} / \mathrm{mol})$ for reaction for selected frames from solvated MD simulation.....

Table 22: Reactant Distances for all the frames ......................................................... 114

Table 23: Transition state Distances for all the frames.................................................. 115

Table 24: Product Distances for all the frames .......................................................... 116

Table 25: Important hydrogen bonding interactions for reactants, transition states and products calculated for selected frames....................................................... 116

Table 26: Optimization script for reactant (or product) structure of $\mathrm{CPD}+\mathrm{HOCl}+$ explicit water molecule ........................................................................... 142

Table 27: Script for scan of potential energy surface to locate structure close to the transition state.

Table 28: Transition state optimization script

Table 29: Single point energy calculation script using implicit solvent environment (PCM), of gas-phase optimized structure of reactant (or product) of CPD + $\mathrm{HOCl}+$ explicit water molecule

Table 30: Parameterization script (QM): Initial partial atomic guess and target dipole calculation

Table 31: Parameterization script (QM): Target water-CPD (H12) interaction energy and distance calculation 150

Table 32: Parameterization script (MM): Water - CPD dipole and optimal interaction energies and distances

Table 33: Frequency calculation script for force constants to get target normal mode frequency and percent contribution

Table 34: Parameterization script (QM): Bond, angle and torsion based on QM calculated force constants for CPD 180

Table 35: Parameterization script (MM): Bond, angle and torsion parameter optimization for CPD.

Table 36: Parameterization script (MM): Potential energy scans of dihedrals and impropers for $\mathrm{CPD}$ 188

Table 37: Initial minimization script for CPD and $\mathrm{HOCl}$ 190 
Table 38: Script for solvating $\mathrm{CPD}$ and $\mathrm{HOCl}$ in a truncated octahedron box..... 192

Table 39: Initial minimization script for solvated CPD and $\mathrm{HOCl}$ 193

Table 40: Initial NVE dynamic simulation script (slowly heating the system to $298 \mathrm{~K}$ ) 195

Table 41: Initial NPT dynamic simulation script 198

Table 42: Script for NPT dynamics simulation used for analysis 200

Table 43: Shell script to run various molecular dynamics scripts via queue on computer cluster and appropriately archive the output files 201

Table 44: Script for reading trajectory and saving coordinates of reactant precursors based on pre-determined criteria 205

Table 45: Script for mapping out minimum energy path using Replica Path method with all MM waters fixed 209

Table 46: Script for locating transition state of system using CI-NEB with all MM waters fixed 212

Table 47: Script for locating transition state using CI-NEB for system with flexible hydration shell

Table 48: QM script read by CHARMM to create actual script to be run by QChem. Used in Replica Path method and CI-NEB method 217

Table 49: Script generated by CHARMM to be run by QChem for each replica in Replica Path method and CI-NEB method 244

Table 50: Topology file for sugars, for n-acytyl-glucosamine and for pyroglutamic acid, which are found in $\mathrm{CPO}$, and for which parameters were created by analogy

Table 51: Patches used to attach sugar-sugar molecules, sugar-amino acids and hemecystine.

Table 52: Lines appended by Cassian to CHARMM parameter file in order to include parameters developed for entries in topology file in Appendix B1 257 


\section{LIST OF FIGURES}

FIGURE

PAGE

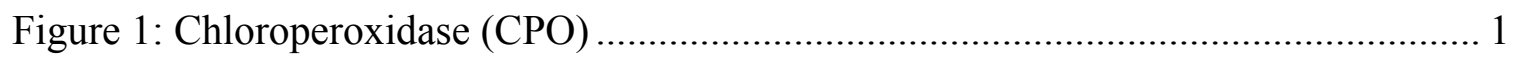

Figure 2: Reaction pathways for different reactions CPO catalyzes ............................... 2

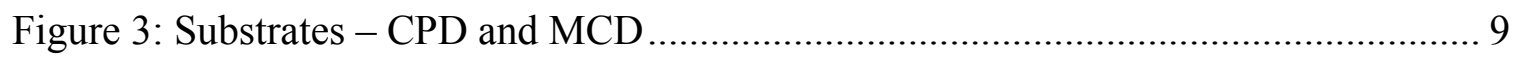

Figure 4: CPO active-site showing CPD and water molecules................................. 9

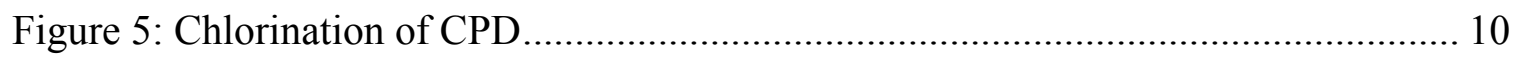

Figure 6: Mechanism of keto-enol tautomerization: (a) acid catalyzed, (b) base catalyzed

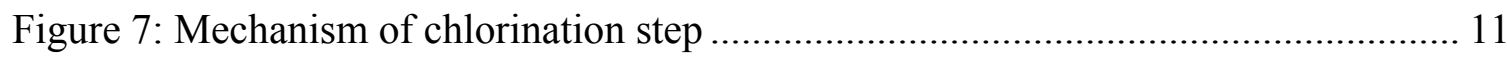

Figure 8: Second step of the chlorination mechanism in a limited hydrated environment. 1

Figure 9: Second step of the chlorination mechanism depicting barrierless reaction......... 1

Figure 10: Bonded and non-bonded interactions in MM.......................................... 19

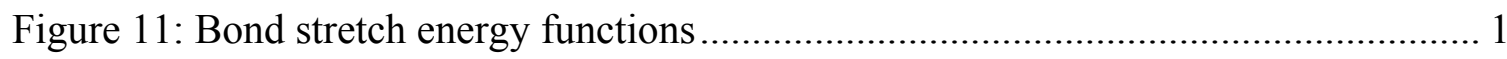

Figure 12: Angle bending energy function ........................................................ 1

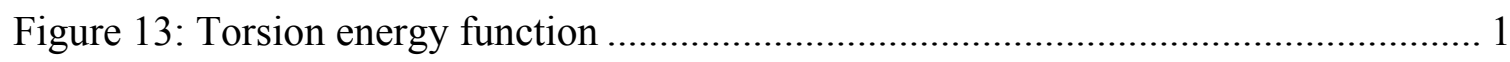

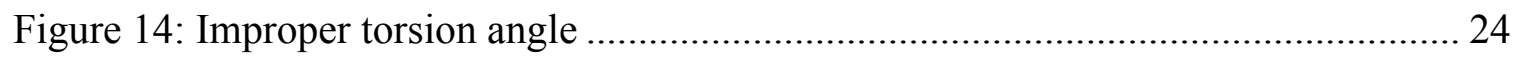

Figure 15: Lennard-Jones potential for van der Waals interaction ................................. 1

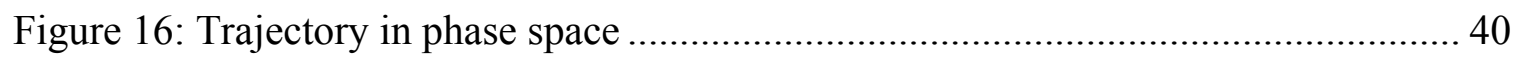

Figure 17: Schematic representation of periodic boundary condition .............................. 42

Figure 18: Solvated $\mathrm{CPD}$ and $\mathrm{HOCl}$ in a truncated octahedron box of water ................. 43

Figure 19: Schematic of QM and MM regions ................................................... 1

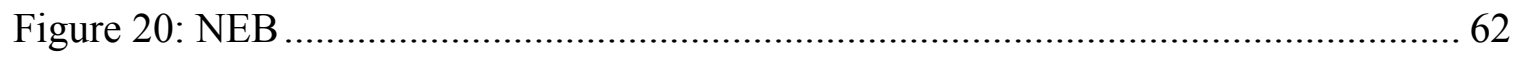

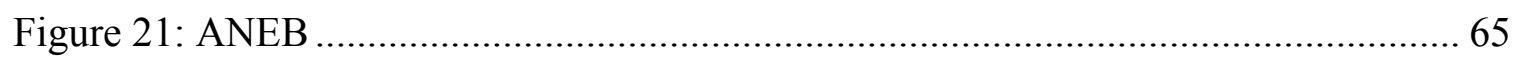

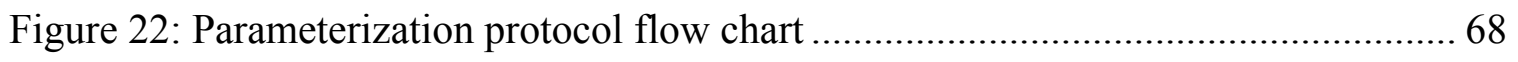




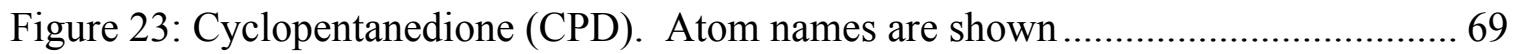

Figure 24: Two example representations of water interacting with atoms in CPD ......... 73

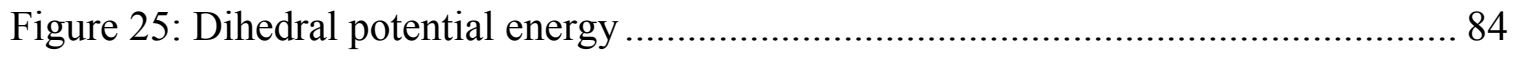

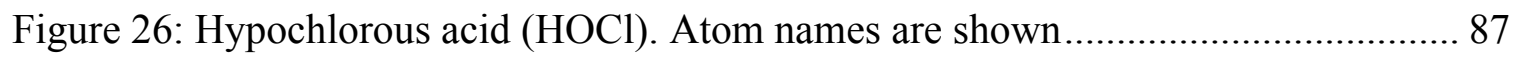

Figure 27: Sugar: (a) alpha-D-glucose (b) alpha-D-xylopyranose .............................. 91

Figure 28: Intramolecular gas phase reaction. Stationary points. $\mathrm{HOCl}$ present to play stabilizing role representative of acidic conditions ..................................... 96

Figure 29: Intermolecular gas phase reaction. Stationary points. $\mathrm{HOCl}$ present to play stabilizing role representative of acidic conditions 97

Figure 30: Stationary points of system with 3 explicit water molecules ........................ 1

Figure 31: Stationary points of system with 5 explicit water molecules ......................... 1

Figure 32: Stationary points of system with 6 explicit water molecules ........................ 1

Figure 33: Stationary points of system with 11 explicit water molecules ....................... 1

Figure 34: Reaction energies and barrier heights for reaction in water clusters................ 1

Figure 35: Representative rare event frame favorable for reaction, chosen from the MD simulation according to the selection criteria. Atoms treated at the QM level in subsequent QM/MM calculations are represented with thick sticks, and MM atoms are represented with thin lines ...................................................... 105

Figure 36: Trajectory frames satisfying the selection criteria. Each vertical group of data points represents one encounter, i.e., all frames lie within $10 \mathrm{ps}$ of the best frame selected. Frames were saved every 20 steps (every $0.02 \mathrm{ps).} 617$ frames satisfied the selection criteria out of a total of 350,000 saved trajectory frames $(0.18 \%)$ 106

Figure 37: Frame 1: Distance change showing the hydrogen abstraction and enolization from reactant to product as the reaction proceeds in the vicinity of the transition state. 108

Figure 38: Optimization of Replica Path Method (SD+ABNR) ................................ 111

Figure 39: Optimization of CI-NEB to locate Transition state (SD+ABNR) ................ 112

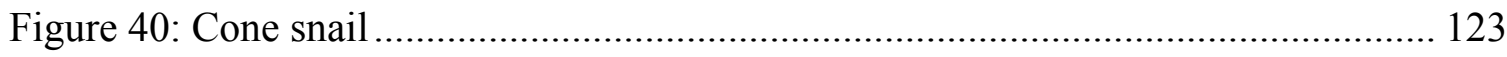




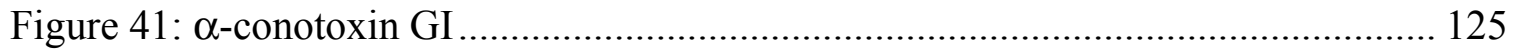

Figure 42: NMR structure showing stable backbone .................................................. 128

Figure 43: distinct structures in solution - Major and Minor ........................................ 128

Figure 44: Simulation of major and minor conformation for 10x10 ns simulation demonstrating that the two structures are stable and distinct

Figure 45: N-H correlation functions. Trajectory frames were reoriented for Major conformation but not for Minor conformation ( $\mathrm{S}^{2}$ values shown elsewhere are based on reoriented data.)....................................................................... 130

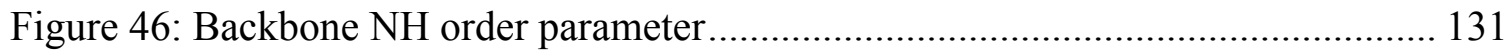

Figure 47: NH bond vector orientation and sampling ................................................ 131

Figure 48: Dihedral $(\Psi)$ of residue 10 and 12 of the major conformation ..................... 132 


\section{LIST OF ACRONYMS}

ACRONYM

ABNR

ACM

AGLC

ANEB

AXYS

BXYS

BARB

BNAG

CC

CI-NEB

$\mathrm{CPO}$

CPD

DFT

DHP

ESP

GGA

GTO

GRMS

HF

IC

IRC
FULL NAME

Adopted Basis Newton Raphson

Adiabatic Connection Method

Alpha-D-Glucose

Adaptive Nudged Elastic Band

Alpha-D-Xylopyranos

Beta-L-Xylopyranos

Beta-L-Arabinose

N-Acetyl-Glucosamine

Coupled Cluster

Climbing Image-Nudged Elastic Band

Chloroperoxidase

1,3 Cyclopentanedione

Density Functional Theory

Dehaloperoxidase

Electrostatic Potential

Generalized Gradient Approximation

Gaussian Type Orbitals

Root Mean Square Gradient

Hartree-Fock

Internal Coordinate

Intrinsic Reaction Coordinate 


\begin{tabular}{|c|c|}
\hline LCAO & Linear Combination of Atomic Orbitals \\
\hline LDA & Local Density Approximation \\
\hline MCD & Monochlorodimedone \\
\hline MD & Molecular Dynamics \\
\hline MEP & Minimum Energy Path \\
\hline MK & Merz-Kollman \\
\hline MM & Molecular Mechanics \\
\hline MO & Molecular Orbitals \\
\hline MP & Moller-Plesset \\
\hline NEB & Nudged Elastic Band \\
\hline NMR & Nuclear Magnetic Resonance \\
\hline PCA & Pyroglutamic Acid \\
\hline PCM & Polarizable Continuum Model \\
\hline PES & Potential Energy Surface \\
\hline QM & Quantum Mechanics \\
\hline $\mathrm{QM} / \mathrm{MM}$ & Quantum Mechanics/Molecular Mechanics \\
\hline RMSD & Root Mean Square Deviation \\
\hline RPATH & Replica Path \\
\hline SD & Steepest Descent \\
\hline STO & Slater Type Orbitals \\
\hline TS & Transition State \\
\hline VDW & van der Waals \\
\hline
\end{tabular}




\section{INTRODUCTION}

\subsection{Chloroperoxidase}

Shaw and Hager [1] reported chlorination activity when extracts of a marine fungus, Caldariomyces fumago, were used to react with $\beta$-ketoadipic acid to give $\delta$ chlorolevulinic acid. Another observation made was that the main metabolite of this organism was a chlorinated species, 2,2-dichloro-1,3-cyclopentanediol, clearly indicating that there was an enzyme or group of enzymes in the organism that facilitated chlorination. It was later discovered by the same group that there were at least four enzymes in the mold extract. The four enzymes identified were an amyloltic enzyme, a catalase, glucose oxidase and another enzyme that they termed Chloroperoxidase (CPO) to denote the activity of the enzyme [2].

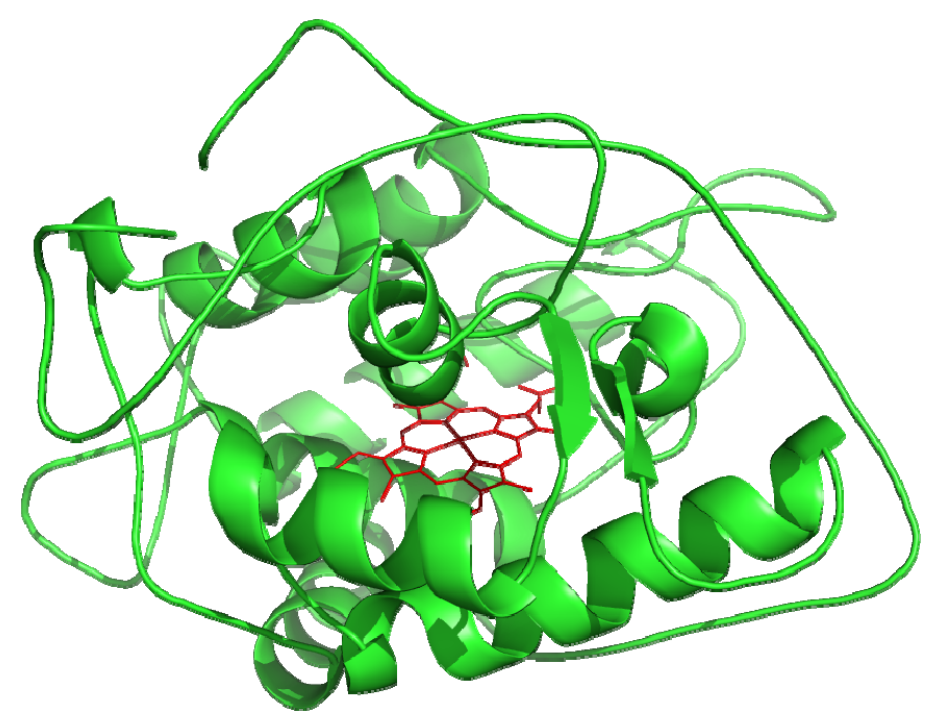

Figure 1: Chloroperoxidase (CPO) 
Chloroperoxidase is a heme containing metalloprotein enzyme. It shares this feature with several other globular enzymes like myoglobin, haemoglobin, cytochrome P450 and horseradish peroxidase. One of the main characteristics distinguishing CPO from most heme enzymes is that the proximal ligand bound to the heme is a cysteine residue [3]. It shares this feature with cytochrome P-450, but a main distinguishing feature between the two is that the distal site of the porphyrin group is charged or polar in CPO and hydrophobic in cytochrome P-450.

Since being discovered more than 50 years ago, CPO has been studied extensively because of its versatility, ecological significance and potential applicability in the pharmaceutical industry [4].

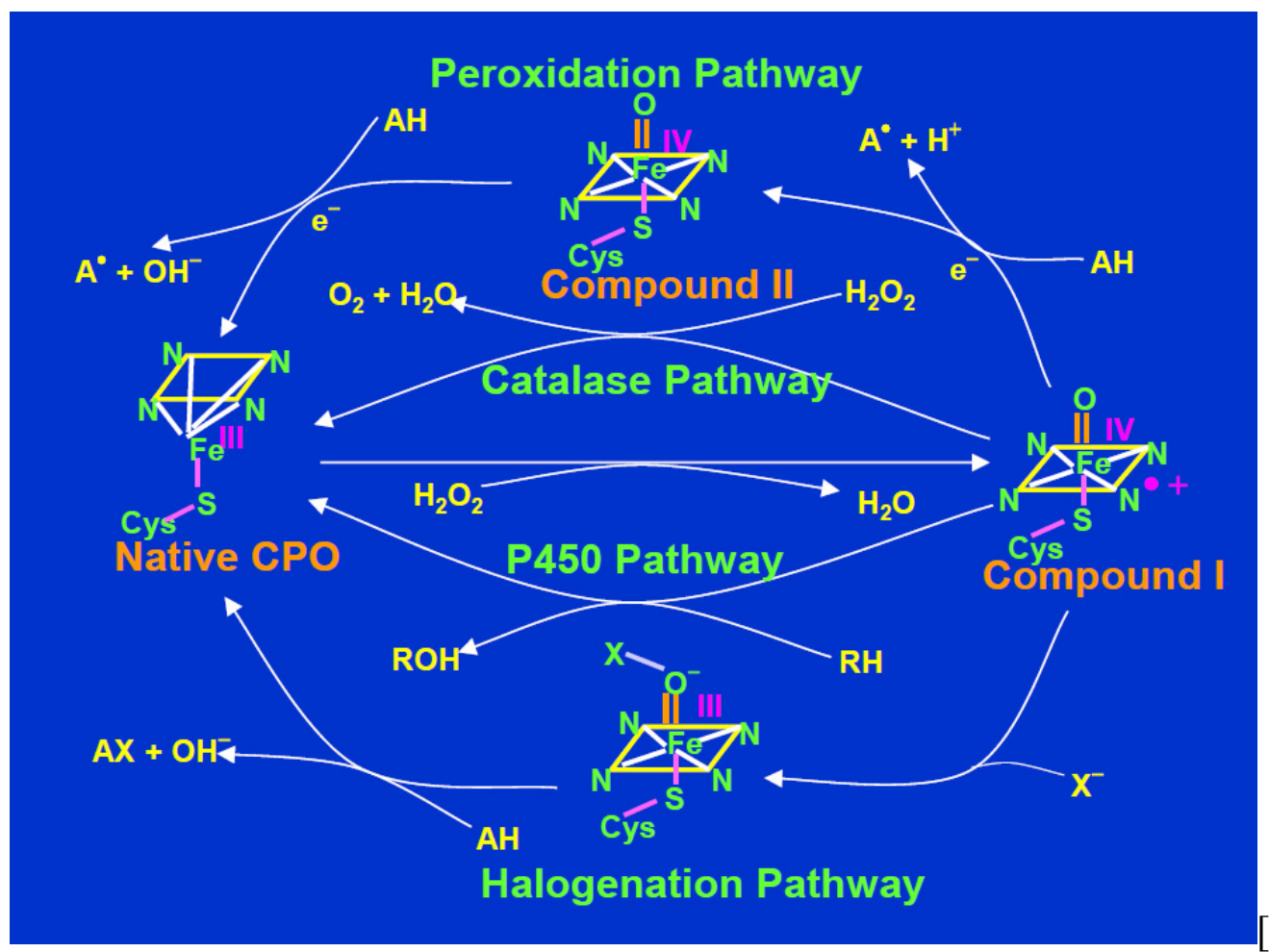

Figure 2: Reaction pathways for different reactions CPO catalyzes 
Chloroperoxidase is known to catalyze several different types of oxidation reactions, summarized in Figure 2. All of these reactions begin with activation of CPO by an oxygen donor. The most commonly used reagent for activating the enzyme is hydrogen peroxide. The unreactive form of the enzyme, called the resting state, has the heme iron in the +3 oxidation state (ferric). Peroxide activates the resting state to a highly reactive state with the iron formally in the +5 oxidation state (perferryl). The active form of the enzyme is called compound I. In fact, it has been shown that a better description of compound I has the iron in the +4 oxidation state (ferryl) and a delocalized radical cation on the porphyrin ring.

\subsubsection{Catalytic Reactions of CPO}

Perhaps the most important of CPO's catalytic reactions is the oxygen insertion reaction, one form of which is epoxidation. Chloroperoxidase has been shown to catalyze the epoxidation of a number of organic substrates. These epoxidation reactions are highly enantiospecific, which makes CPO a pharmaceutically significant enzyme with substantial biotechnological potential. It is this potential combined with the ease of manufacture and availability of CPO that has driven the study of this reaction over the past several years. Much has been learned about the reaction mechanism of this reaction. Modification of the histidine residue at the distal site inhibits the epoxidation reaction, suggesting that the reaction occurs near the distal site of the heme. Other catalytic reactions compete with the epoxidation reaction. The most important competitor is the 
catalase reaction, which involves the dismutation of hydrogen peroxide to give water and an oxygen atom. The catalase reaction is much faster than the epoxidation reaction and depletes the hydrogen peroxide. The active site near the heme is only accessible via two narrow channels. One possible reason that the catalase reaction can out-compete the epoxidation reaction is that the substrates for the epoxidation reaction have to travel through the narrow channel to reach the active site. One line of research is aimed at controlling or inhibiting the catalase activity. It was discovered that there are different optimal $\mathrm{pH}$ ranges for different substrates and that $\mathrm{pH} 5.5-6.0$ diminishes the catalase activity while not affecting the epoxidation reaction greatly. There is, however, a risk of deactivating $\mathrm{CPO}$ at the high end of this $\mathrm{pH}$ range. Using an alkyl peroxidase instead of hydrogen peroxidase as the oxygen donor slows down the catalatic activity, but the alkyl peroxide tends to lower the stability of Chloroperoxidase. Other mutation studies are being performed to discover motifs that would reduce catalase activity while maintaining or improving the epoxidation turnover rates.

Chloroperoxidase also catalyzes the dehydrogenation of substrates that is characteristic of heme peroxidase enzymes $[6,7]$. The mechanism for this reaction is not completely understood. It is thought that for most, though not necessarily all, substrates the peroxidase reaction occurs at the surface of the enzyme and not near the heme active site. Like the epoxidation reaction, the peroxidase reaction also competes with the catalase reaction.

Another reason CPO is extensively studied is that it shares structural and functional features with several important enzymes, most importantly cytochrome P-450 and the thyroid enzyme involved in the iodonization process. Chloroperoxidase is more 
easily produced and activated than these enzymes. Structure and reactivity can be studied using $\mathrm{CPO}$, and the findings can be extrapolated to these other enzymes to better understand how they function.

The most prominent of CPO's catalytic reactions is the chlorination reaction, which has ecological implications [8-14]. In addition to the chlorination reaction, CPO also catalyzes other halogenation reactions including those of iodine and bromine, but not fluorine. The enzymatic chlorination reaction occurs at low $\mathrm{pH}$. There was early concern [9] that the global prevalence of organohalogens was mainly due to anthropogenic contributions. However, several experiments have been able to show that there is significant contribution from biological activity to the global organohalogen burden. Given that CPO is easily accessible and found in nature, it was the enzyme of choice used by many research groups to demonstrate the possibility of enzymatic catalysis of chlorination of organic compounds found in plant litter, fluvic acid, lignin structures and humic substances found in soil samples. There are also examples of plant matter still attached to trees that contains organohalogens originating from degradation, presumably from microbial or fungal attacks [13]. The source of the halogen and oxidizer could be the plant itself, which tends to produce hydrogen peroxidase in cell walls [13]. There are several organisms like fungi, algae and bacteria that produce similar enzymes that would explain the presence of organohalogens in soils, surface water and volatile halogenated anisoles found on surface of oceans [14]. 


\subsubsection{Previous Work on Mechanism of CPO-catalyzed Halogenation Reaction}

Given the versatility and potential economic significance of CPO, there is little wonder that it has been studied extensively over the years. However, its most prominent reaction, chlorination, is still not completely understood. One postulated mechanism involves catalytic formation of $\mathrm{HOCl}$ at the active site of $\mathrm{CPO}$ followed by release into solution, where it halogenates substrates $[4,15-21]$. The other postulated mechanism involves the chlorination of substrates at the heme active site [22-30]. Studies have been performed to support either of these two postulates, but it has been difficult for a consensus to be reached.

The non-active site hypothesis is based on the observation that most of the chlorinated substrate products show no enantiomeric excesses; that some substrates are too large to access the active site; and that $\mathrm{HOCl}$ and $\mathrm{Cl}_{2}$, which can chlorinate any of the substrates, are observed in solution in chlorination reactions involving CPO.

The active site hypothesis is based on the following experiments. (1) There is a preference for certain substrates over others when the reaction is carried out using a mixture of substrates. For example, the preference for thiourea and methionine over monochlorodimedone (MCD) is 50:1 and 30:1, respectively, for the enzymatic reaction. By comparison, the preference for thiourea over $\mathrm{MCD}$ is $2: 1$ when $\mathrm{HOCl}$ is directly added to the reaction mixture in the absence of $\mathrm{CPO}$, and $3: 1$ when $\mathrm{Cl}_{2}$ is directly added. There is no preference for methionine over $\mathrm{MCD}$ using either $\mathrm{HOCl}$ or $\mathrm{Cl}_{2}$ directly [24]. (2) The rates of chlorination of some substrates are higher for the reactions in the presence of CPO than for reactions involving chemical controls [23]. (3) Woggon et al. 
developed synthetic active-site analogs of $\mathrm{CPO}$ for which the formation of stable intermediates with $\mathrm{OCl}^{-}$and $\mathrm{HOCl}$ bound to the heme was demonstrated. These were presumed to be the intermediates through which the enzyme chlorinates the substrates [27-31]. (4) Some steady state kinetic studies suggest that chlorination via the free $\mathrm{HOCl}$ mechanism is unlikely. The studies show that the production of $\mathrm{HOCl}$ and $\mathrm{Cl}_{2}$ at the enzyme active site and the subsequent release into solution is a slow process that may be considered as competing with the chlorinating reaction $[22,23]$. (5) Some substrates give different product distributions in the enzyme catalyzed reaction and the chemical control reaction. For example, while no appreciable stereoselectivity is observed for most chlorinated substrates, for 2methyl-4-propyl-cyclopentane-1,3-dione it was reported that the enzyme catalyzed reaction results in a product, 2-chloro-2-methyl-4-propylcyclopentane-1,3-dione, with a 40:60 ratio of the racemic diastereomers, while the chemical control gives a 55:45 ratio of the racemic diastereomers [26]. (6) There is also a reported case of stereoselective halohydration of glycals and steroids using CPO [32-35].

Thus the experimental evidence regarding the mechanism of enzymatic halogenation by $\mathrm{CPO}$ is inconclusive. Some consider the general lack of enantiospecificity to be the most persuasive evidence and conclude that halogenation occurs outside the active site. However, the many observations favoring active-site halogenation leave the case far from clear. The bulk of the work presented in this dissertation is aimed at contributing to the resolution of this dispute. 


\subsubsection{Substrate Used to Study Reaction}

In the research presented here, I employ a variety of theoretical approaches to study the chlorination mechanism of $\mathrm{CPO}$, and I choose a particular model substrate, 1,3 cyclopentanedione (CPD), for consideration. This substrate was chosen for several reasons. First, $\mathrm{CPD}$ is a cyclic $\beta$-diketone, and it is known that cyclic $\beta$-diketones, $\beta$-keto acids, and substituted phenols make good halogen acceptors. Second, 2,2-dichloro-1,3cyclopentanediol was found to be the main metabolite of CPO [1]. CPD has been shown to be catalyzed to 2,2-dichloro-1,3-cyclopentanedione [36]. CPD Third, CPO and another cyclic $\beta$-diketone, monochlorodimedone (MCD), are the compounds most widely used for monitoring the halogenation activity of CPO spectroscopically. The intermediate species in the reaction of CPD to the dichloro metabolite is a monochloro species, 2chloro-1,3-cyclopentanedione [36]. It is easy to observe the conversion of CPD to the metabolite because CPD and the intermediate have absorption maxima at 242 and 248 $\mathrm{nm}$, respectively, with a high extinction coefficient while the metabolite has an absorption maximum at $277 \mathrm{~nm}$ with a very low extinction coefficient [37]. Monochlorodimedone is more commonly used because it is readily available, and it is easy to observe the conversion from MCD to dichlorodimedone [37]. For computational studies, though CPD is preferable to MCD because it contains fewer heavy atoms and is hence less demanding of computational resources. Finally, a crystal structure of CPO with CPD at its active site has been reported [38]. This structure will be useful for future studies of halogenation at the active site. 

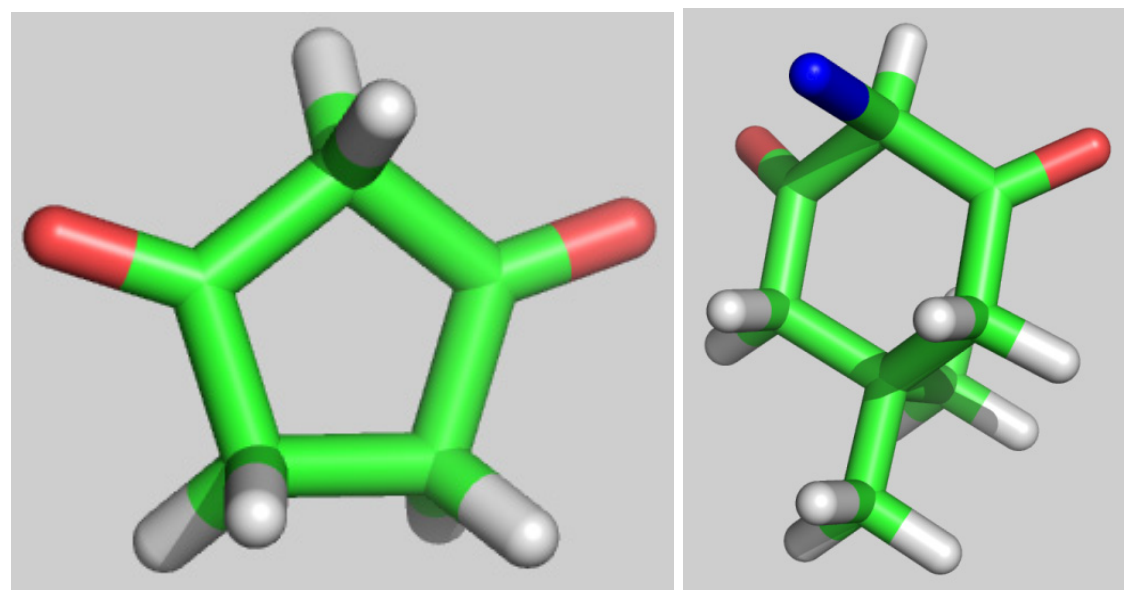

Figure 3: Substrates - CPD and MCD
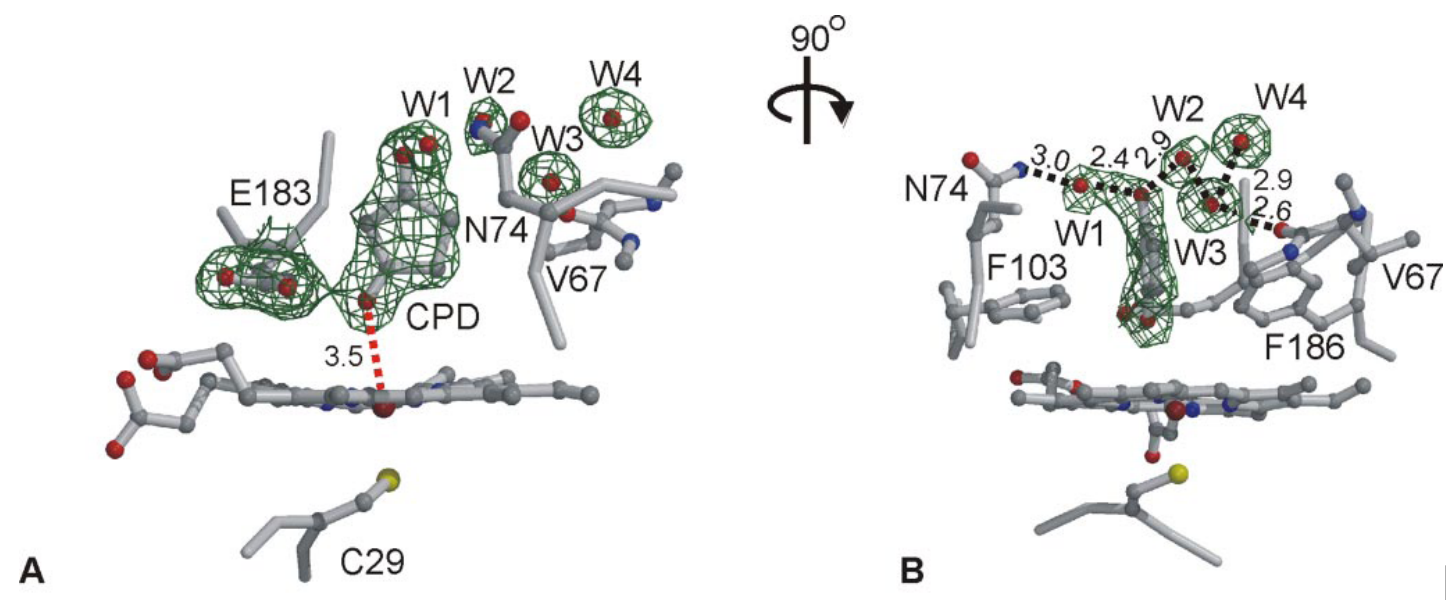

Figure 4: CPO active-site showing CPD and water molecules

\subsection{Chlorination Mechanism of CPO}

It is well known that ketones in aqueous solution can undergo an internal rearrangement in which a hydrogen is transferred from the $\alpha$-carbon to the keto oxygen and the double bond shifts from $\mathrm{C}=\mathrm{O}$ to $\mathrm{C}=\mathrm{C}$. The resulting species is called an enol because it contains both -ene (carbon-carbon double bond) and alcohol (OH) groups. 
The two forms are called tautomers, and the process of interconversion is tautomerization. In aqueous solution, both tautomers are present in equilibrium. An alternative nomenclature for the tautomers of $\beta$-diketones is diketo and keto-enol.

The overall process of the chlorination reaction, with keto-enol tautomerization as the first step, is depicted in Figure 5. The mechanisms of the individual steps are shown in Figure 6 and Figure 7. The chlorination (or more generally, the halogenation) of ketones begins with tautomerization to the enol form. Electrophilic attack of a chloronium $\left(\mathrm{Cl}^{+}\right)$on the carbon-carbon double bond of the enol form, followed by transfer of the hydroxy proton to a solvent water, yields the chlorinated product. The tautomerization step can be either acid- or base-catalyzed, as shown in Figure 6 . I consider acid catalysis because CPO-catalyzed chlorination is carried out at low $\mathrm{pH}(\mathrm{pH}$ $3-4)$.

keto-enol tautomerization

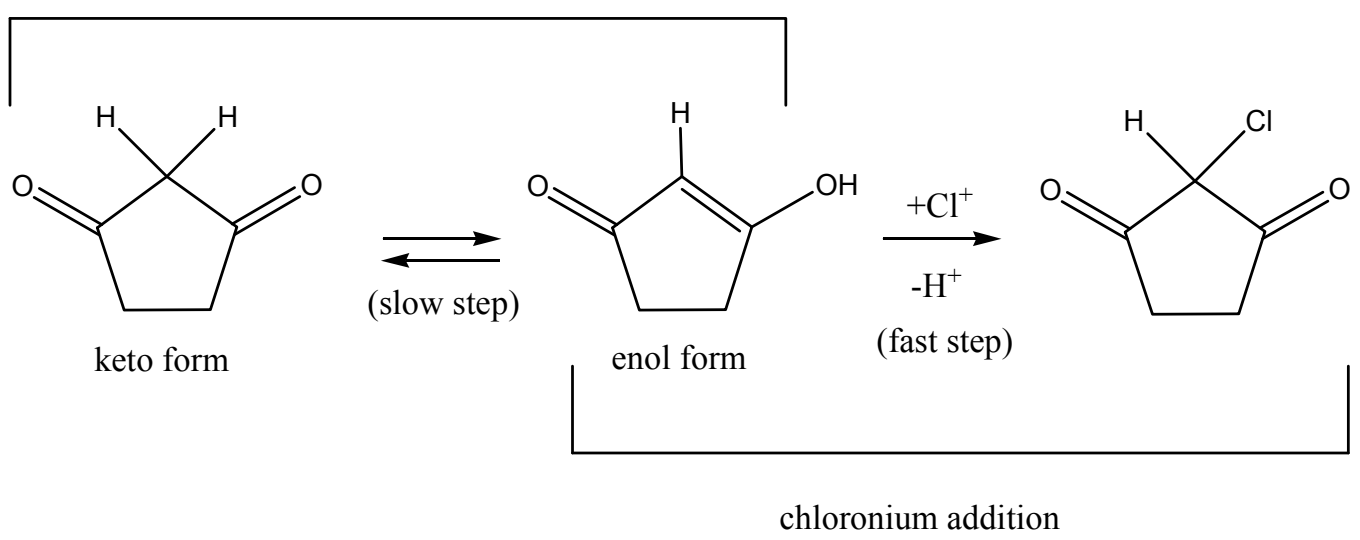

Figure 5: Chlorination of CPD 

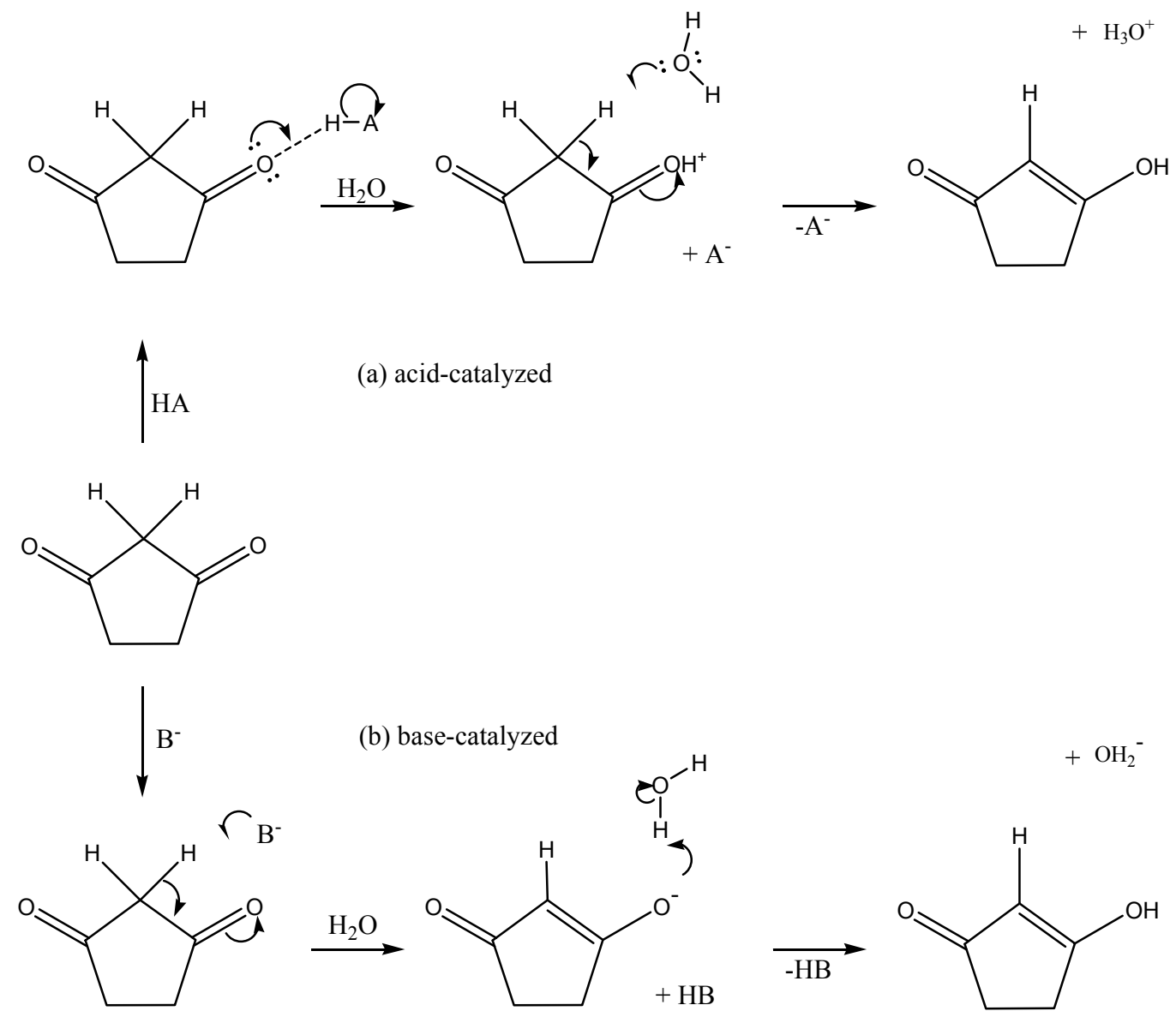

Figure 6: Mechanism of keto-enol tautomerization: (a) acid catalyzed, (b) base catalyzed

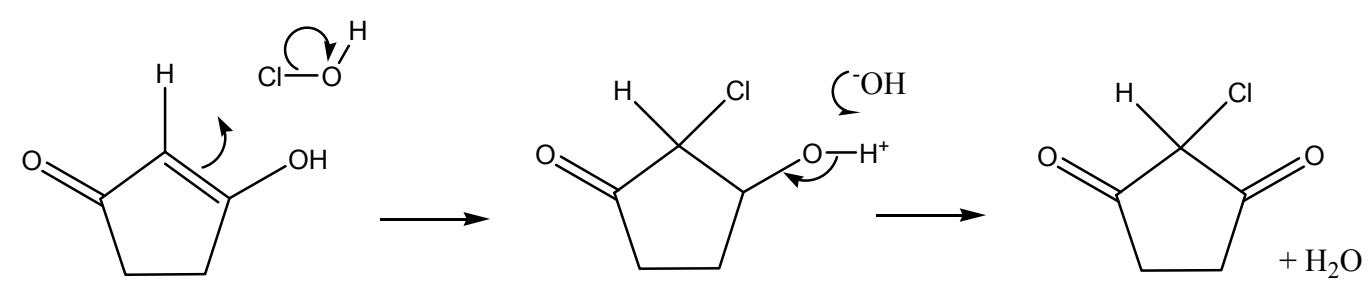

Figure 7: Mechanism of chlorination step

For the overall process of the chlorination reaction shown in Figure 5, the second step is expected to be fast and barrierless. The mechanism of this step is depicted in Figure 7. I modeled the chlorination step in a limited hydration environment, with 14 
water molecules, using the quantum mechanics approach explained in section 2.4. The data in Figure 9 verifies that the reaction shown in Figure 8 is nearly barrierless and hence a kinetically fast reaction. Therefore, I focus the study on the rate-limiting step which is the acid catalyzed keto-enol tautomerization.

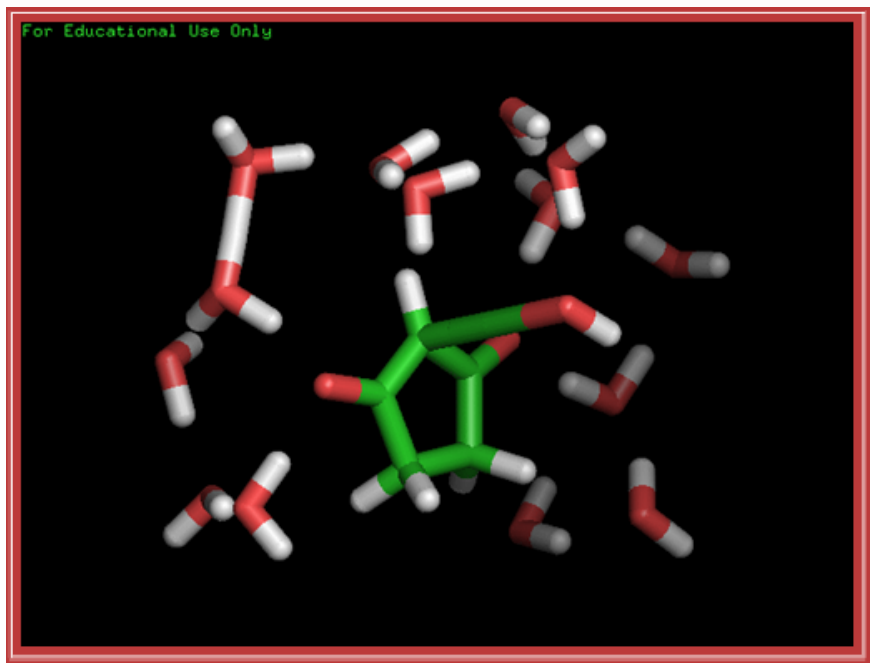

Figure 8: Second step of the chlorination mechanism in a limited hydrated environment

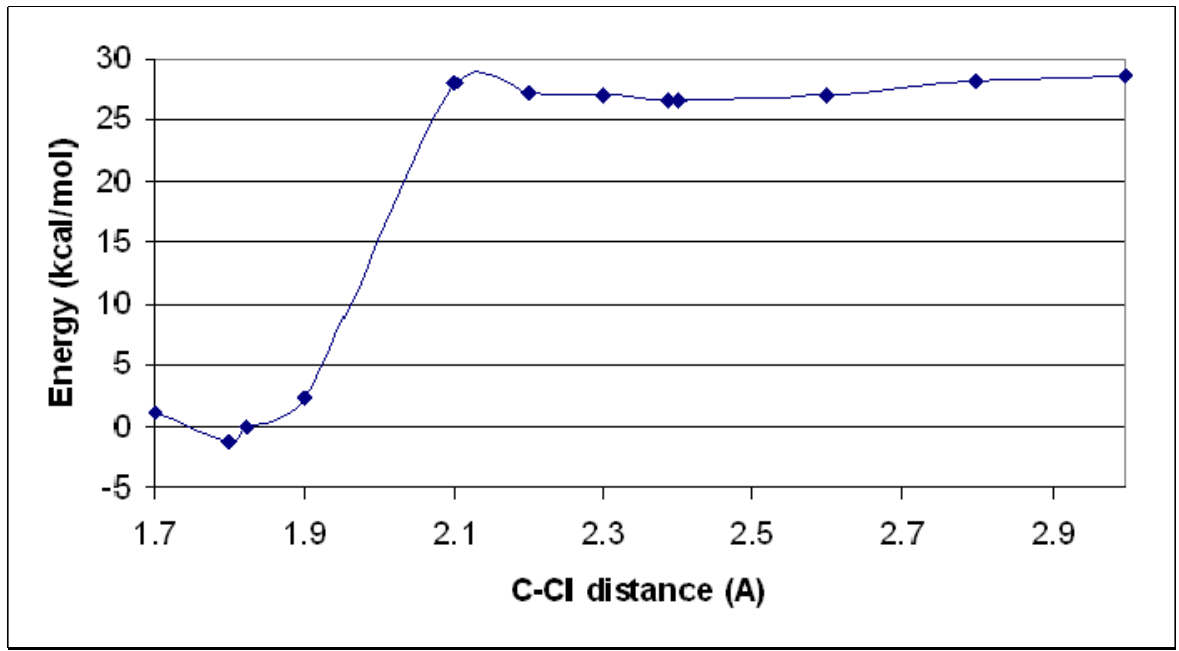

Figure 9: Second step of the chlorination mechanism depicting barrierless reaction 


\subsection{Previous theoretical studies of keto-enol tautomerization}

Solvation plays a critical role in many reactions including keto-enol tautomerization and will be the focus of my studies on the chlorination mechanism of CPD. Considerable prior theoretical work has been done on the role of water in keto-enol tautomerization. Here this prior work is summarized.

The equilibrium between the keto and enol tautomers in solution has been studied with implicit solvent methods, with reasonable success, reproducing $\log \mathrm{K}$, where $\mathrm{K}$ is the equilibrium constant, to within $\sim \pm 0.2$ in many cases [39-41]. However, such methods are not sufficient for modeling the kinetics of tautomerization in aqueous solution because the mechanism explicitly involves water molecules.

Calculations determine the barrier for keto-enol tautomerization via intramolecular proton transfer to be generally in the range $60-85 \mathrm{kcal} / \mathrm{mol}$, depending on the compound. This is much larger than the experimental reaction barriers [42-44]. Inclusion of a single explicit catalytic water lowers the barrier by $30-40 \mathrm{kcal} / \mathrm{mol}$ for typical molecules. The mechanism is a two-step process with the water first abstracting a proton from the alpha carbon, and subsequently donating a proton to the keto oxygen. Ma et al. determined that a second catalytic water lowers the tautomerization barrier for 3-methyl-5-hydroxyisoxazole a further $8 \mathrm{kcal} / \mathrm{mol}$ [43]. Freitag et al. showed that for malonaldehyde, a third explicit water with a solvating but not catalytic role can lower the barrier another $7 \mathrm{kcal} / \mathrm{mol}$ [44]. Further solvating waters had decreasing incremental effects. These studies demonstrate that inclusion of explicit water molecules is crucial 
for modeling the kinetics of keto-enol tautomerization, but the degree of explicit solvation required for an accurate model is not known.

Cucinotta et al. applied an ab initio metadynamics treatment to keto-enol tautomerization of acetone in aqueous solution [45]. They found that a single water lowers the free energy barrier from 57.7 to $46.1 \mathrm{kcal} / \mathrm{mol}$, and a solution environment of 28 waters lowers the barrier further to $38.5 \mathrm{kcal} / \mathrm{mol}$. In solution, a Grotthuss mechanism involving four water molecules in a proton transfer chain was observed.

With the exception of the metadynamics work, all the studies focused on optimized structures with up to two catalytic waters, and in one case with up to three further waters that were solvating but not catalytic. It is unknown whether this degree of hydration is sufficient. More fundamentally, the assumption is made that barriers for fully optimized structures involving multiple waters can accurately predict experimental activation energies. However, such structures might represent reaction events so extremely rare that they do not predict the observed kinetics well. I address these issues with two studies on CPD. In the first study, I systematically increase the number of explicit waters in fully optimized structures and determine the barrier and the reaction energy as functions of the number of waters. I find that the barrier to reaction continues to decrease as the number of waters increases beyond five. In the second study, I use classical molecular dynamics to generate solution environments representative of $300 \mathrm{~K}$. I then determine reaction barriers from snapshot structures using a hybrid QM/MM method, allowing one or two water molecules to be explicitly active. I find that the instantaneous environment has a significant effect on the barriers. This result, ultimately, 
is consistent with Cucinotta's metadynamics work [45] but provides complementary detail on the nature of the influence of water on this keto-enol tautomeric reaction.

The energies of the stationary points (reactant, product and transition state) were calculated in different environments as described above. I start with the simplest system consisting of only the $\mathrm{CPD}$ and $\mathrm{HOCl}$ molecules, with or without a single catalytic water, in the gas phase. I then focus most of the study on systems consisting of CPD, $\mathrm{HOCl}$ and one reactive/catalytic water molecule in different environments.

\subsection{Solvation}

Based on previous theoretical work on keto-enol tautomerization it is clear that solvation effects are quite substantial and critical, as they influence the reaction barrier strongly. Both at the active site and in aqueous solution, water molecules are present and will interact with the substrate during reaction. Water itself, in fact, plays a catalytic role in the reaction. Furthermore, water molecules not directly involved in the breaking and forming of chemical bonds may also influence the reaction strongly through hydrogen bonding, in addition to less directional dielectric effects that water exerts. Thus we will find that the way in which water is incorporated into the reaction model is critical. The most basic way to represent solvent in theoretical calculations, as a dielectric continuum, turns out to be entirely inadequate in this case.

In reaction at the active site, a limited number of waters are available to interact with the substrate. This is apparent from the crystal structure shown in Figure 4, which 
identifies four waters quite near a CPD molecule bound at the active site. These four waters are resolved in the X-ray structures because they have strong stabilizing interactions with neighboring moieties. In reality, there may be other waters in addition to these four, but the total number is limited by the size and shape of the active site pocket. Thus in the context of the active site, it is misleading to speak of solvation because the number of waters is limited. In this work, I will refer to such a water environment as "limited hydration." In contrast I will refer to the water environment of CPD dissolved directly in aqueous solution as "full solvation."

The central work in this dissertation reports on two investigations, one of the chlorination reactions in "limited hydration" conditions and the other chlorination reaction in "full solvation" conditions. Quite different approaches are used for the two investigations. The limited hydration work connects with previous theoretical work reported on keto-enol tautomerization, which is a critical step in the reaction (see below). Keto-enol tautomerization is a fundamental concept in organic chemistry, but the influence of the water environment on the reaction is still not fully understood. My work on limited hydration suggests that previous work on keto-enol tautomerization needs to be understood in a more restricted way than that work made clear. The energetics worked out in the previous work is representative of the particular limited hydration structures used, but not representative of limited hydration in general and certainly not representative of full solvation.

My work on the reaction in "full solvation" at near-physiological temperature leads to a paradoxical observation. The dominant reaction events have energetic barriers very similar to those encountered under extremely limited hydration conditions, namely a 
single water that assumes a catalytic role. This similarity in barriers turns out to be a consequence of equivalent degrees of reactant- and transition-state stabilization by other waters in the full-solvation calculations. The full-solvation barriers are quite different from the barriers for limited hydration with several waters present.

The work on the chlorination reaction presented here is restricted to the various water environments just described. This turned out to be rich and difficult undertaking in itself. Reaction of CPD situated within the enzyme $\mathrm{CPO}$ at the active site is a subject for future work. The work presented here demonstrates that inclusion of hydrating waters will be critical for modeling the reaction at the active site. Implications of this work extend beyond $\mathrm{CPO}$, as many chemical reactions are likely to be sensitive to the nature of the limited-hydration environment found at an active site.

The rest of this dissertation is organized as follows. Chapter 2 contains an outline of theoretical methods used (content on basic quantum mechanics and molecular mechanics is from standard computational chemistry texts $[46,47])$. Part of the work presented is based on molecular mechanics models. This necessitated the development of such models for CPD and other molecules used. This is an involved process of parameterization, and it is detailed in Chapter 3. Chapter 4 presents the research on the chlorination reaction in limited hydration conditions representative of the active site environment. Chapter 5 presents the research on the chlorination reaction in fully solvated conditions representative of aqueous solution. Chapter 6 presents the major conclusions regarding the halogenation mechanism. Chapter 7 briefly describes contributions to three other research projects. The appendix contains the most important scripts used for running the calculations. 


\section{METHODS}

\subsection{Molecular Mechanics}

The term Molecular Mechanics (MM), which was first introduced in 1970, describes the application of classical mechanics in the determination of molecular structures at equilibrium. In the simplest MM treatment of molecules, the atoms are treated as balls and the bonds as springs connecting them. These springs follow Hooke's law, which is a representation of a harmonic oscillator. The application of classical mechanics to the motion of these balls (or atoms) bound by springs (or bonds) is the basis of molecular mechanics. Thinking of molecules as being made up of balls and springs dates back to as early as 1930 and was first suggested by Andrews [48, 49].

It might seem as if the laws of quantum mechanics are ignored with this treatment of molecules. In reality, though, the force fields are based on experimental and quantum mechanical calculations, so quantum mechanics is incorporated in the force field. The MM treatment of molecules works quite well as long as the structures do not deviate too much from their respective equilibrium structures. The harmonic oscillator approximation of the bonds often provides sufficiently accurate results. However, bond breaking or forming cannot be modeled using molecular mechanics.

Quantum Mechanics (QM) cannot be used to model large molecules such as proteins, which number in the thousands of atoms due to computational costs. The MM models use a simplistic representation of atoms in comparison to quantum mechanics to allow one to 
study systems with large numbers of atoms with reasonable accuracy. Molecular mechanics is used extensively in the study of biomolecular systems like docking for drug design, protein folding, binding constants and reaction precursors.

One of the main objectives of a good force field is transferability from molecule to molecule. For example, we would like to be able to apply the force field derived from one molecule to another similar molecule to correctly predict the minimum energy structure for the new molecule. This key development in MM, where it was possible to transfer force constants between alkanes was first made by Snyder and Schachtschneider [50].

The functional forms used for developing force fields are described below. The various types of interactions in a macromolecule can be classified as bonded interactions and non-bonded interactions. The potential energy contributions from the various interactions are combined to give the total potential energy of the macromolecule. Each contribution is described separately below.

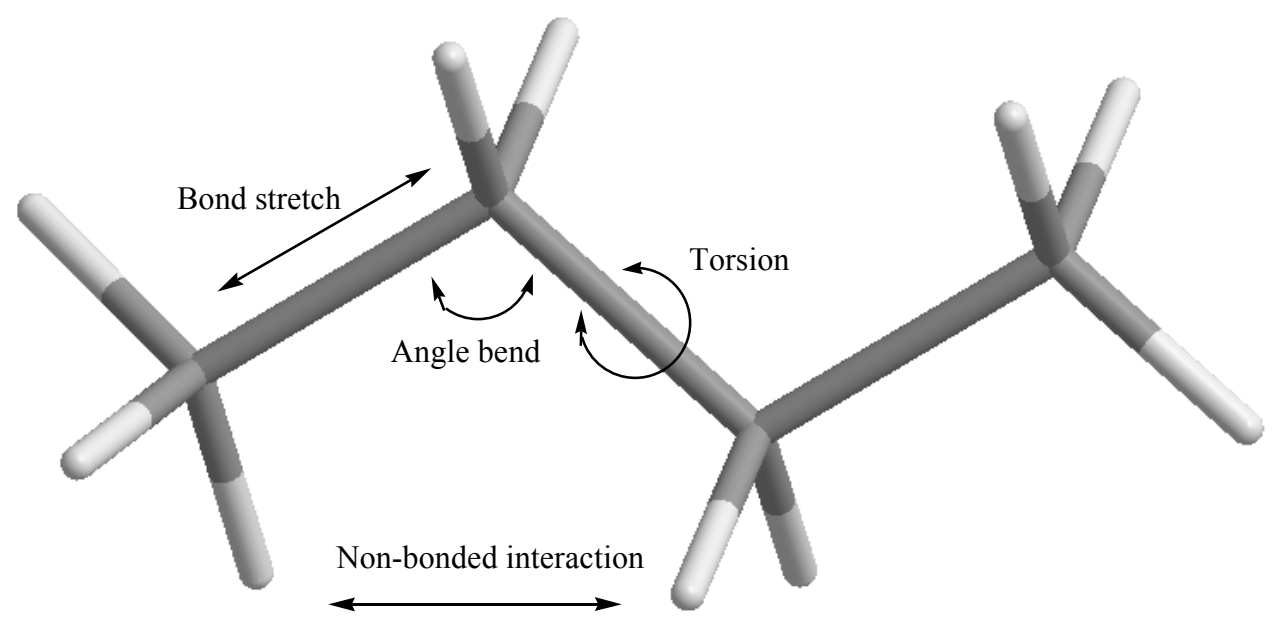

Figure 10: Bonded and non-bonded interactions in MM 
The program I use for the MM model is CHARMM [51]. CHARMM is a molecular simulation program developed to study large, many-atom structures usually encountered in biological systems, for example proteins, peptides, nucleic acids, carbohydrates and organic drug-like molecules or ligands in solution, crystal or membrane environments. CHARMM provides computational tools to perform molecular energy minimization, molecular dynamics, analysis and path sampling. It can be interfaced with several QM software packages to study systems that require the hybrid QM/MM method explained in section 2.5 .

\subsubsection{Bonded Interactions}

\section{Bond Stretching}

To determine the potential energy contribution from bond stretching we consider the ball and spring model. The force due to the spring is given by Hooke's Law

$$
F_{s}=-k_{s}\left(R-R_{e}\right)
$$

The potential energy is given by

$$
U\left(r_{A B}\right)=-\int F\left(r_{A B}\right) d r
$$


Applying a Taylor expansion we obtain

$$
U(r)=U\left(r_{e q}\right)+\left.\frac{d U}{d r}\right|_{r=r_{e q}}\left(r-r_{e q}\right)+\left.\frac{1}{2 !} \frac{d^{2} U}{d r^{2}}\right|_{r=r_{e q}}\left(r-r_{e q}\right)^{2}+\left.\frac{1}{3 !} \frac{d^{3} U}{d r^{3}}\right|_{r=r_{e q}}\left(r-r_{e q}\right)^{3}+\ldots .
$$

We label the equilibrium bond distance $r_{\mathrm{eq}}$. We can, for convenience, consider the minimum of the functional form to be zero. Therefore, the first and second terms in the Taylor's expansion are zero. The simplest contribution then is from the first non-zero term which is the quadratic term. This approximation may be sufficient for small stretching, but as the bond stretches far from the equilibrium values the potential energy contribution becomes infinitely positive, which is unrealistic, and the harmonic approximation fails. This failure is inherent in truncating the Taylor's expansion, so the logical thing would be to include more terms. When the next cubic term is included, it causes an unintended divergence to negative infinity as the bond is stretched further. Therefore, the quartic term also has to be included.

Alternately, the functional form of the Morse potential can be used which describes the bond stretch rather accurately.

$$
U\left(r_{A B}\right)=D_{A B}\left[1-e^{-\alpha_{A B}\left(r_{A B}-r_{A B, e q}\right)}\right]^{2}
$$

However, the exponential term in the Morse potential is computationally less efficient for calculation than the truncated polynomial terms from the harmonic oscillator. In most force fields some variation of the following bond stretching term is used 


$$
U\left(r_{A B}\right)=\frac{1}{2}\left[k_{A B}+k_{A B}^{(3)}\left(r_{A B}-r_{A B, e q}\right)+k_{A B}^{(4)}\left(r_{A B}-r_{A B, e q}\right)^{2}+\ldots \ldots\right]\left(r_{A B}-r_{A B, e q}\right)^{2}
$$

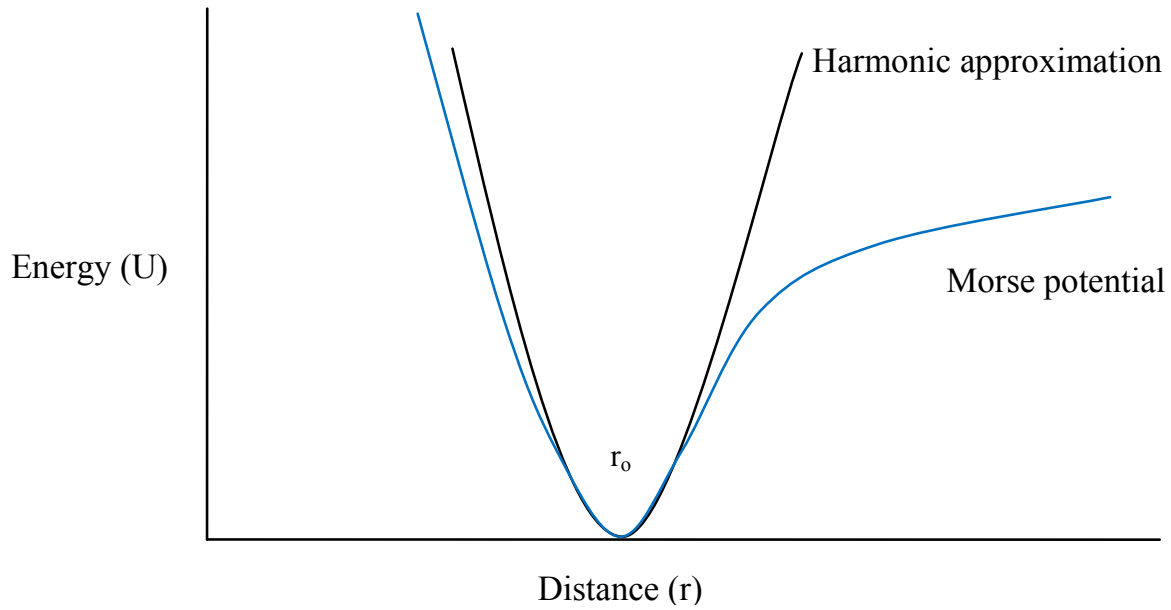

Figure 11: Bond stretch energy functions

\section{Angle Bending}

As with bond stretching, angle bending or bond angle deformation can be modeled using the harmonic approximation about the equilibrium bond angle. It has an important contribution to the total potential energy as evidenced by vibrational spectroscopy data that show energy variation attributed to bond bending. The same considerations as for bond stretching apply to the functional form, so a generally useful function form is

$$
U\left(\theta_{A B C}\right)=\frac{1}{2}\left[k_{A B C}+k_{A B C}^{(3)}\left(\theta_{A B C}-\theta_{A B C, e q}\right)+k_{A B C}^{(4)}\left(\theta_{A B C}-\theta_{A B C, e q}\right)^{2}+\ldots \ldots . .\left(\theta_{A B C}-\theta_{A B C, e q}\right)^{2}\right.
$$




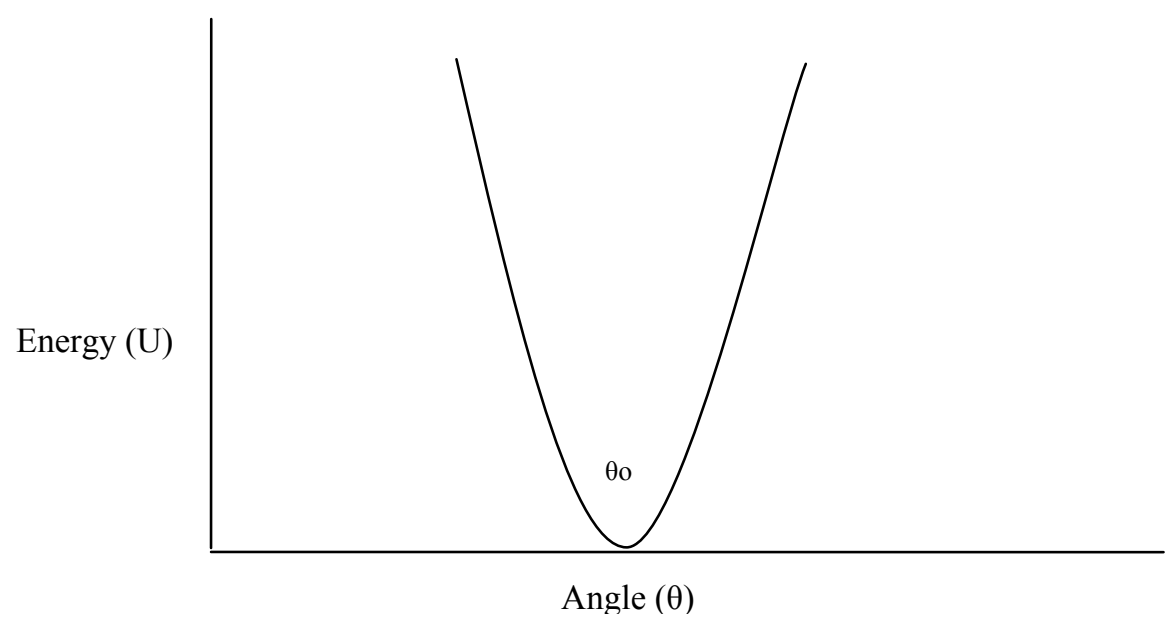

Figure 12: Angle bending energy function

\section{Torsions}

Torsional motion is periodic, so its contribution to the potential energy is also periodic. It is conveniently modeled using a cosine function, or more generally a sum of cosines to allow for irregularity in the periodic function. Energy contribution due to dihedral (torsional) motion involving four atoms is given by the following expression.

$$
U\left(\omega_{A B C D}\right)=\frac{1}{2} \sum_{\{j\}_{A B C D}} V_{j, A B C D}\left[+(-1)^{j+1} \cos \left(j \omega_{A B C D}+\psi_{j, A B C D}\right)\right]
$$




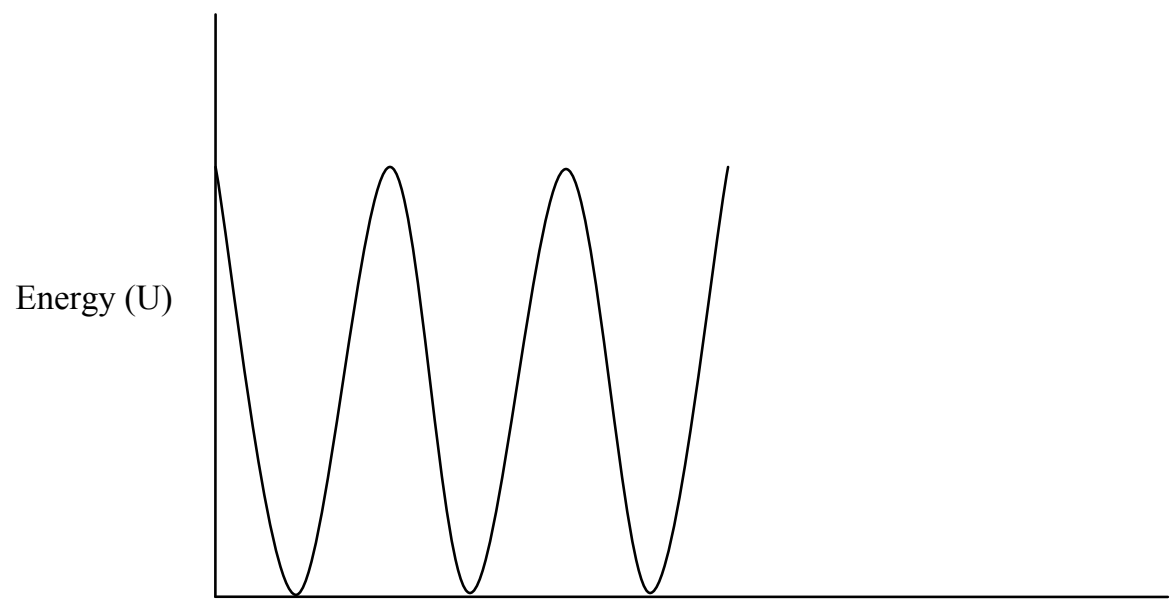

Torsion angle $(\omega)$

Figure 13: Torsion energy function

\section{Improper torsions}

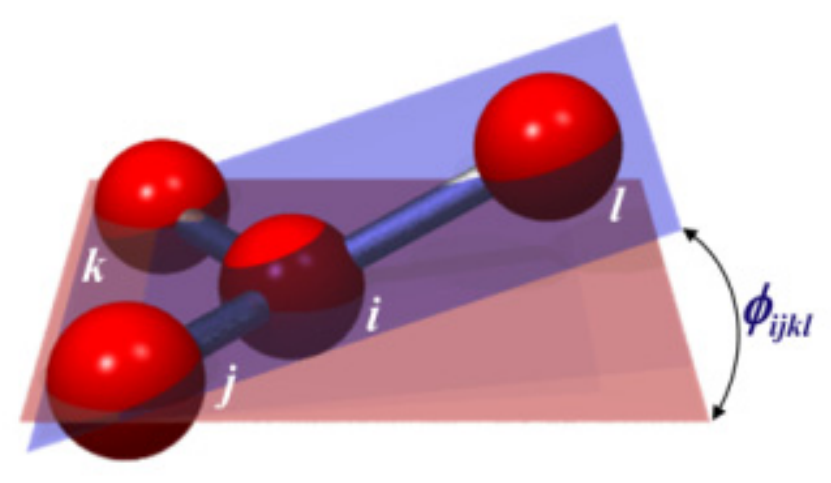

Figure 14: Improper torsion angle

Improper torsions are a special situation that involves four atoms, but unlike the case with regular torsions, for improper torsions there is a central atom bonded to the three other atoms. If we define a plane by the central atom and two other atoms that are bonded to it, then the angle the fourth atom creates to that plane is the improper torsion angle. Improper torsion terms are included only when the other energy terms are 
insufficient to maintain correct geometries. Specifically, improper torsion terms are used to maintain puckering in structures. Improper torsional energy contributions are only accounted for by CHARMM if there are explicit parameters for them for a specific molecule. Improper torsion terms are not included in the energy by default like the other bonded interaction terms. The CHARMM topology file must specify that a particular improper torsion term be included.

\subsubsection{Non-bonded Interactions}

As stated earlier, one of the main objectives in developing a force field is the transferability of the force field to similar groups of molecules. During the initial development of force fields it was observed that 'transferability' was attainable by including non-bonded interactions, at least of the nearest neighbors. This is why nonbonded interaction contributions are critical and have to be included. The following two types of non-bonded interactions are included in most force fields, while some force fields include additional interactions.

\section{Van der Waals Interactions}

Van der Waals force is the attractive forces experienced by non-bonded neutral atoms. As neutral atoms gets in close vicinity to each other they experience attractive forces due to the induced dipoles. These short distance intermolecular forces are weaker 
than a chemical bond, but are important when atoms get close enough like in liquids and solids and are responsible for properties like surface tension and capillary action.

In the simplest case we have two atoms, represented as hard spheres, each with a characteristic radius. We also assume they have no charge. At infinite separation, there is no interaction and hence the contribution to the potential energy is zero. As they approach each other the two spheres eventually touch. At this point they cannot approach each other and the energy contribution tends to infinity. Realistically, we know from QM that this is a simplistic case and in fact as the atoms approach each other there is an attractive force due to the electronic correlation, also called dispersion. As they come closer, at some very short distance, the energy contribution tends to rise exponentially to infinity. The attractive energy is inversely proportional to $r^{6}$. This dispersion interaction between atoms is usually modeled by the Lennard-Jones potential and is given below.

$$
U\left(r_{A B}\right)=4 \varepsilon_{A B}\left[\left(\frac{\sigma_{A B}}{r_{A B}}\right)^{12}-\left(\frac{\sigma_{A B}}{r_{A B}}\right)^{6}\right]
$$

It would be more accurate to represent the repulsion with an exponential term instead of the inverse $\mathrm{r}^{12}$ term. But, exponential calculations are more difficult on the computer in comparison to a polynomial term, which is why the inverse $\mathrm{r}^{12}$ term is used instead. This is an empirical result and used for computational convenience. 


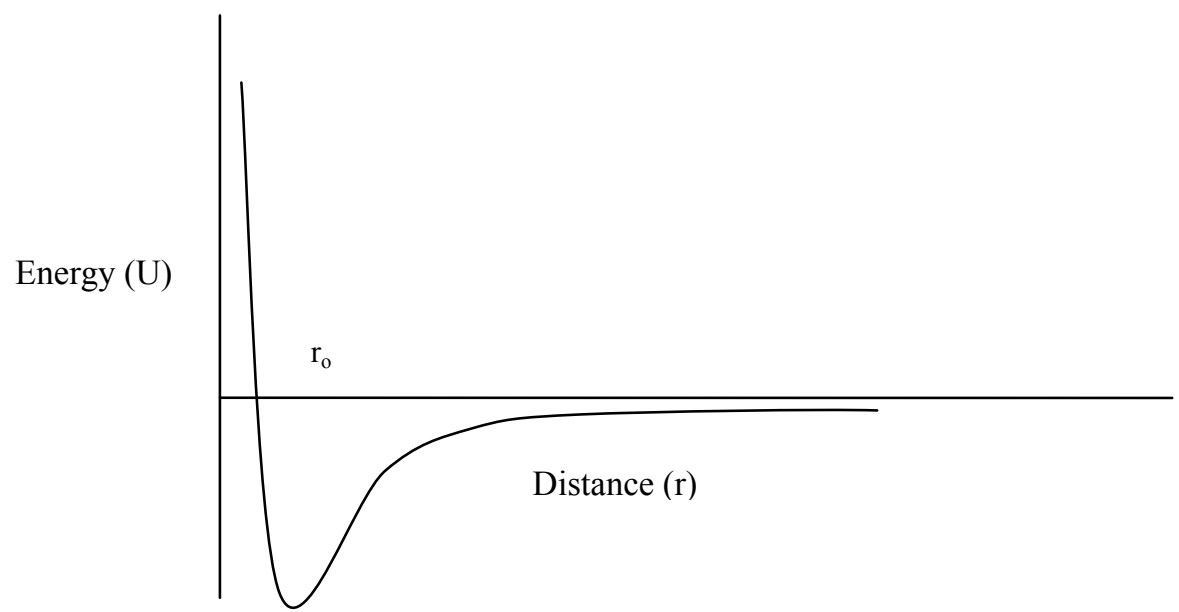

Figure 15: Lennard-Jones potential for van der Waals interaction

\section{Electrostatic Interactions}

Electrostatic interactions are the energy contribution that arises from non-bonded interactions between atoms that have a charge associated with them. Again, if we consider the simplest case, we assume a fixed point charge associated with an atom. We then apply Coulomb's Law to determine the energy contribution which is given by the following equation.

$$
U_{A B}=\frac{q_{A} q_{B}}{\varepsilon_{A B} r_{A B}}
$$

We know that as atoms move the effective atomic charges will respond to the environment, that is to say, atomic systems are polarizable. When permanent charges are assigned, the polarizability is neglected. However, the fixed permanent charges used are generally capable of reproducing several experimental observable or QM computed 
properties quite accurately. Development of polarizable force fields is an active topic of research and there are several groups working on developing polarizable force fields. However, the community has not yet embraced any particular polarizable force field.

The calculations of non-bonded interactions are the most time consuming part of the energy evaluations since it would mean calculation for every pair of atoms in the system $\left(\mathrm{N}^{2} / 2\right.$ interactions, where $\mathrm{N}$ is the number of atoms). These interactions are distant dependent and two atoms that are quite far from each other may have minimal contribution to the total energy. An implementation consideration then is how far we want to calculate non-bonded interactions. In interest of saving computation time a truncation scheme is used. A cutoff distance is set so that non-bonded interactions are only calculated if an atom is within a certain distance from another atom. However, this introduces a discontinuity in the energy evaluations. Consider for example, two atoms that are exactly at the cutoff distance when the energy contribution is calculated. At the next step, they move just beyond the cutoff distance from each other, thus the new energy contribution calculated is precisely zero. The small step would cause a drastic difference in the energy evaluation. To avoid this abrupt discontinuity, a switching function is used to gradually exclude the non-bonded interactions instead of abruptly. For example, if the cutoff was set at $12 \AA$, one can use a switching function to gradually exclude the nonbonded interactions from $12 \AA$ to $14 \AA$ instead of abruptly setting it to zero at $12 \AA$. The preferred method of treating electrostatic interactions is the Particle Mesh Ewald summation method. It is a method to calculate the electrostatic interactions in an infinite lattice or under periodic boundary conditions (explained in section 2.3.3). 


\subsubsection{Solvation}

Modeling gas phase reactions are relatively simple. However, all biochemical processes occur in the condensed phase and the chemical reaction we study occurs in solution. Therefore, it is critical to model a solvation environment around the molecule of interest. A solvation environment model can be broadly classified as an implicit model or explicit model.

In an implicit solvent model, also called the continuum solvation model, the environment is like a continuous medium with a dielectric constant representing the solvent and properties consistent with those of the solvent. To represent water as the extended solvent environment we use the dielectric constant of water $(\varepsilon=78.4)$. This kind of representation of the solvent has the advantage that the computation overhead is low and used with QM model. I used the Polarizable Continuum Model (PCM) method as implemented in Gaussian98 [53]. The PCM model is a widely used implicit solvent model that represents the environment of the system by an dielectric continuum having the dielectric constant of the solvent. However, an implicit model is only used when the condensed phase does not play a critical role in the reaction environment.

An explicit solvent model includes explicit solvent molecules. Adding explicit water molecules can get prohibitively expensive in the quantum mechanics model. At best it is feasible to include a single solvation shell. However including explicit water molecules is quite feasible in the molecular mechanics model which is routinely done while studying large biomolecular systems. I use both types of explicit solvated environments. 


\subsection{Geometry optimization}

One of the key motivations of $\mathrm{MM}$ is to find minimum energy structures or equilibrium structures that are representative of realistic molecules. Locating minimum energy structures constitutes a mathematical problem and the general field of study is called optimization theory. One of the priorities is to facilitate geometry optimization by simplifying the functional forms without compromising accuracy. One such example is using polynomial terms instead of exponential terms to facilitate computation. There are several algorithms that have been developed that are used in achieving this goal. Obviously, some are more efficient that others and in most cases it is best to use a combination of different algorithms.

Consider a molecule with $\mathrm{N}$ atoms. This molecule has $3 \mathrm{~N}$ degrees of freedom, of which six are translational and rotational (or five if it is a linear molecule). The remaining $3 \mathrm{~N}-6$ (or $3 \mathrm{~N}-5$ ) degrees of freedom are vibrational degrees of freedom and therefore, there are $3 \mathrm{~N}-6$ independent variables that describe them which are also called internal coordinates.

All these variables can be written as a matrix

$$
q=\left(\begin{array}{l}
q_{1} \\
q_{1} \\
q_{3} \\
\cdots \\
q_{p}
\end{array}\right)
$$


The simplest way to optimize would be to optimize the energy for one variable at a time. This is called a line search, but when we have several variables and if they are correlated this approach becomes inefficient.

Another approach would be to proceed in a direction that minimizes the total energy with respect to all the variables at the same time. The first derivative matrix, also called the gradient and shown below, is needed. The optimization process proceeds by altering the coordinates so as to modify the structure in the negative direction of $\mathbf{g}(\mathbf{q})$ until a local minimum is reached. This is a first order approach.

$$
g(q)=\left(\begin{array}{l}
\frac{\partial U}{\partial q_{1}} \\
\frac{\partial U}{\partial q_{2}} \\
\cdots \\
\frac{\partial U}{\partial q_{p}}
\end{array}\right)
$$

Steepest Decent methods, which was introduced by Wiberg in 1965 [54], and Conjugate Gradient methods, which were introduced by Fletcher and Reeves [55], are examples of first derivative methods. Such methods get us close to the minimum quickly, but an inherent drawback of such an approach is the convergence. It can sometimes be hard for such algorithms to converge to the minimum. One usually starts an optimization process with such an algorithm to get close to the minimum. Then one uses a more sophisticated method, usually a second order method, to finally converge to the minimum. 
Second order methods make use of the second derivative of the energy function, also called the Hessian.

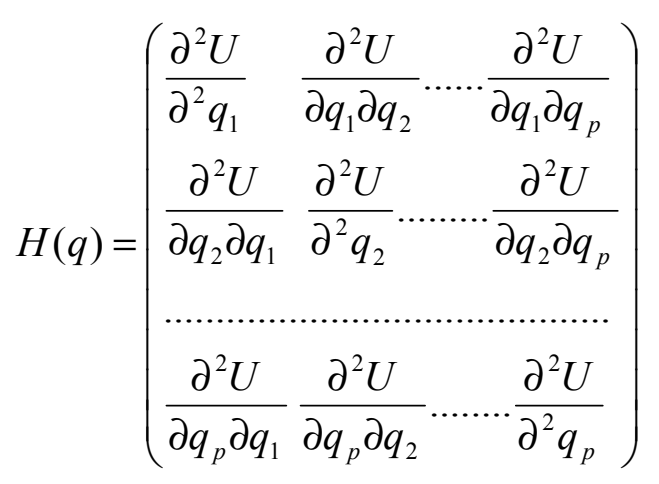

If we substitute in the Taylor's expansion series of energy we obtain

$$
\begin{gathered}
U(q)-U\left(q_{e q}\right)=\sum_{i=1}^{p} \xi_{i}\left(\frac{\partial U}{\partial q_{i}}\right)_{\xi_{i}=0}+\frac{1}{2} \sum_{i=1}^{p} \sum_{j=1}^{p} \xi_{i} \xi_{j}\left(\frac{\partial^{2} U}{\partial q_{i} \partial q_{j}}\right)_{\xi_{i}=0, \xi_{j}=0}+\ldots \ldots . \\
U(q)-U\left(q_{e q}\right)=\xi^{T} g+\frac{1}{2} \xi^{T} H \xi+\ldots \ldots
\end{gathered}
$$

Second order methods tend to converge to the minimum quickly if they are close to the minimum to begin with. However, they are computationally more expensive because of the necessity of calculating the Hessian, especially if needed at every step. In practice, it is necessary to calculate the Hessian for the first step when using a second order method, but some algorithms use an approximate Hessian for subsequent steps to make the 
calculation computational efficient. However, in some sensitive cases it is essential to calculate the Hessian for every step, which is the basis for the Newton-Raphson method.

The minimization algorithms used in this project were a combination of first order and second order methods. Minimization was initiated using Steepest Descent followed by the Adopted Basis Newton Raphson (ABNR) method. ABNR is a quasi-NewtonRaphson method.

\subsection{Molecular Dynamics}

The most popular application of Molecular Mechanics is optimization of a structure to find its minimum energy or an equilibrium structure using the laws of classical mechanics. While this is quite useful, it is even more interesting to predict molecular properties computationally that may or may not be feasible to measure experimentally. One technique that is used to do this computationally is called Molecular Dynamics (MD).

\subsubsection{Phase Space}

In order to describe MD, I will first discuss the concept of phase, and then show that $\mathrm{MD}$ is a way to sample phase space in order to compute average properties. The state of a macromolecule can be completely described using classical laws by specifying the position and momenta of all the particles that make up the macromolecule. A system 
made up of $\mathrm{N}$ particles would need $6 \mathrm{~N}$ coordinates to describe the macromolecule, $3 \mathrm{~N}$ coordinate to describe position along the $\mathrm{x}, \mathrm{y}$ and $\mathrm{z}$ axes and $3 \mathrm{~N}$ coordinates to describe their corresponding momenta along the respective axes. The resulting $6 \mathrm{~N}$ dimensional space is called the 'phase space' of the macromolecule. At any given instant in time, the macromolecule occupies one point in phase space. Over time, a system maps out a trajectory in phase space. Molecular dynamics is a technique used to construct the phasespace trajectory of a macromolecule, given a set of initial conditions.

Phase space point

$$
\begin{gathered}
X=(q, p) \\
q=\left(x_{1}, y_{1}, z_{1}, x_{2}, y_{2}, z_{2}, \ldots \ldots . .\right) \\
p=\left(p_{x, 1}, p_{y, 1}, p_{z, 1}, p_{x, 2}, p_{y, 2}, p_{z, 2}, \ldots \ldots . .\right)
\end{gathered}
$$

Properties measured are averages over the states of the system. Phase-space is extremely large and encompasses every possible state of the system, even those that the system is unlikely to sample, like unrealistic high energy states. In other words, phase space includes states that have a very low probability of being visited in reality. Thus phase-space is essentially a large wasteland that mostly contains states that are unlikely to contribute much to the expectation or average value of a particular property. Hence, it 
makes sense to use available computational resources efficiently by only mapping out trajectories that concentrate on regions of phase space the system would be most likely to sample. These regions would likely be near local minima or equilibrium structures. For this reason, most MD simulations are preceded by short energy minimization simulations. (Another reason MD simulations are preceded by a short energy minimization is that most structure coordinates come from x-ray crystallography. The crystal structures usually contain bad contacts between atoms with artificially high forces. If we start the MD simulation with these high forces, the trajectory may be pushed away from the local minima into high-energy, improbable regions of phase space.)

Experimental measurements of properties are averages over states of a system, as mentioned above. Often the system of interest is microscopic or submicroscopic, yet the experimental conditions include a macroscopic sample containing many "copies" of the system. For example in a solution containing a mole of enzyme molecules in which case Avogadro's number of enzyme molecules is present, each capable of sampling a different state of the enzyme. In this case, an experimental measurement of some property of the enzyme will represent an ensemble average over many copies. (Experimental measurements will also be time averaged if the measurement takes longer than the characteristic time associated with the property in question, but the important point here is the ensemble-average nature of most experiments). An MD trajectory provides a sampling over time of a single copy (or small number of copies) of the system. The connection between simulation and experiment rests on the assumption that the timeaverage over the trajectory of a single copy of the system will give the same results as the ensemble average over many copies of the . This assumption is the ergodic theorem. It 
allows us to make a connection between simulation and experiment, provided the simulation is long enough for sufficient sampling of phase space.

An interesting aspect of a phase-space point is that the location of the next point can easily be determined from the current position and momentum. The relationship between two positions and the momentum over some time interval is given by

$$
q\left(t_{2}\right)=q\left(t_{1}\right)+\int_{t_{1}}^{t_{2}} \frac{p(t)}{m} d t
$$

The relationship between momenta and acceleration is given by

$$
p\left(t_{2}\right)=p\left(t_{1}\right)+m \int_{t_{1}}^{t_{2}} a(t) d t
$$

The acceleration $a(t)$ is given by

$$
a=\frac{F}{m}
$$

The acceleration can be calculated from the forces acting on each particle via the force field. Hence, we can map out the trajectory in phase-space in the forward and reverse directions once we have a single phase-space point. 
For non-analytical systems the above expression can be re-written using Euler's approximation in the limit $\Delta \mathrm{t} \rightarrow 0$. We get the same result if we use the Taylor's expansion truncated at second order.

$$
\begin{gathered}
q(t+\Delta t)=q(t)+\frac{p(t)}{m} \Delta t \\
p(t+\Delta t)=p(t)+m a(t) \Delta t
\end{gathered}
$$

\subsubsection{Verlet-leapfrog algorithm}

On the basis of the above general discussion of phase space we now consider the algorithm used to map the trajectory of the system through phase space. One way to map out the trajectory is by using the Verlet algorithm. Consider time steps of $\Delta t$ in the forward and reverse direction.

The expression for position in the forward direction

$$
q(t+\Delta t)=q(t)+\left(\frac{d q}{d t}\right)_{t} \Delta t+\frac{1}{2 !}\left(\frac{d^{2} q}{d t^{2}}\right)_{t}(\Delta t)^{2}+\ldots \ldots
$$

The expression for position in the reverse direction 


$$
q(t-\Delta t)=q(t)-\left(\frac{d q}{d t}\right)_{t} \Delta t+\frac{1}{2 !}\left(\frac{d^{2} q}{d t^{2}}\right)_{t}(\Delta t)^{2}+\ldots
$$

If we add the two expressions we get

$$
\begin{gathered}
q(t+\Delta t)=2 q(t)-q(t-\Delta t)+\frac{1}{2} a_{t}(\Delta t)^{2} \\
a_{t}=\left(\frac{d^{2} q}{d t^{2}}\right)_{t}
\end{gathered}
$$

We can see that to get the next position, after a time interval of $\Delta \mathrm{t}$, all we need are the current and previous position and the acceleration, which we can get from the force field.

$$
\begin{gathered}
a=\frac{F}{m} \\
F=-\frac{d V}{d q}
\end{gathered}
$$

The Verlet algorithm allows us to propagate through phase-space with no explicit reference to velocity or momentum. However, in most cases it is useful to run simulations at a particular temperature or gradually to be able to change temperature. Temperature is directly related to random velocity, so one needs to be able to manipulate velocity directly in order to control the temperature of the simulation. The leapfrog algorithm, a 
modification to the Verlet algorithm, was developed to couple the propagation of position and velocity directly.

$$
\begin{aligned}
& q\left(t+\frac{\Delta t}{2}+\frac{\Delta t}{2}\right)=q\left(t+\frac{\Delta t}{2}\right)+\frac{d q\left(t+\frac{\Delta t}{2}\right)}{d t} \frac{\Delta t}{2}+\frac{1}{2 !} \frac{d^{2} q\left(t+\frac{\Delta t}{2}\right)}{d t^{2}}\left(\frac{\Delta t}{2}\right)^{2}+\ldots . \\
& q\left(t+\frac{\Delta t}{2}-\frac{\Delta t}{2}\right)=q\left(t+\frac{\Delta t}{2}\right)-\frac{d q\left(t+\frac{\Delta t}{2}\right)}{d t} \frac{\Delta t}{2}+\frac{1}{2 !} \frac{d^{2} q\left(t+\frac{\Delta t}{2}\right)}{d t^{2}}\left(\frac{\Delta t}{2}\right)^{2}+\ldots .
\end{aligned}
$$

Subtracting we get

$$
\begin{gathered}
q(t+\Delta t)=q(t)+v\left(t+\frac{\Delta t}{2}\right) \Delta t \\
v\left(t+\frac{\Delta t}{2}\right)=\frac{d q\left(t+\frac{\Delta t}{2}\right)}{d t}
\end{gathered}
$$

For $v(t)$ we obtain

$$
v\left(t+\frac{\Delta t}{2}\right)=v\left(t-\frac{\Delta t}{2}\right)+a(t) \Delta t
$$

From the above two expressions we see that for half a time step $(\Delta t / 2)$ we evaluate velocity and then for the second half $(\Delta t)$ we evaluate the new position. The new 
position is calculated based on the current position at time $t$ and velocity at time step $\Delta t / 2$. The velocity at time step $\Delta \mathrm{t} / 2$ in turn is based on the previous velocity at time step $-\Delta \mathrm{t} / 2$ of the particle and the current acceleration of the particle at time t.

An important consideration that has to be made is the time interval $\Delta \mathrm{t}$ used for computation. If the time step is too large we will deviate from the true phase-space trajectory, since we take a step in the direction tangent to the true trajectory i.e. constant acceleration. However, if we take time steps that are too small, we will end up with very short trajectories through time that will sample too small a region of phase-space to be useful. Practically, a time step one or two orders of magnitude smaller than the highest frequency motion generally yields a sufficiently accurate trajectory. The highest frequency motion in atomic systems is the vibrational motion of bonds between a heavy atom and a hydrogen atom, which has a period of about $10^{-14} \mathrm{~s}$. In practice, time steps of 0.5-1.0 fs are recommended for most purposes.

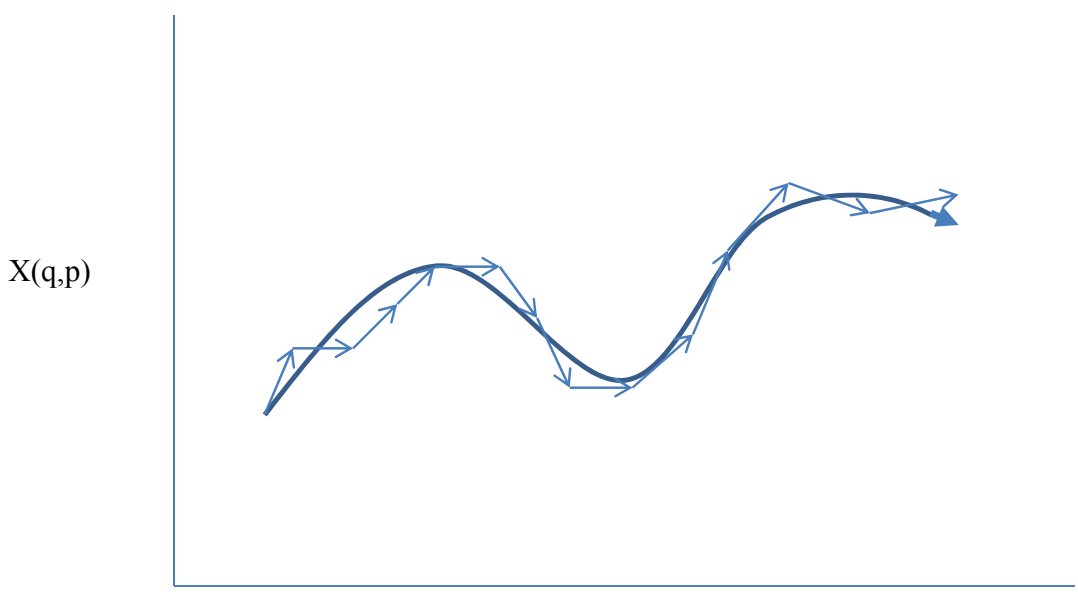

Time

Figure 16: Trajectory in phase space 
Another option to allow the use of larger time steps is to freeze the highest frequency motion. This can be done by constraining the heavy atom - hydrogen bond length. The keyword in CHARMM to constrain the heavy atom - hydrogen atom bond is called SHAKE. The next highest frequency motion would then be a heavy atom - heavy atom vibration with a frequency typically a factor of $2-5$ smaller in magnitude. This approach can only be used if the property being studied is independent of those degrees of freedom which are removed. In addition to increasing the time step by a factor of two 2 (time step of $2 \mathrm{fs}$ ), reduction in the number of degree of freedom provides some saving in time and integration stability.

\subsubsection{Periodic Boundary Conditions}

A molecule being modeled is placed in a box of finite size. This model presents a technical difficulty. The size of the box cannot be very large because of the computational cost of including a large number of atoms is great, especially if the box is filled with water. If the box is too small then the surface effects of the box may affect the chemistry of the system. Beyond the edge of the box is essentially vacuum. Both these effects are unrealistic.

One way to handle this situation is to use Periodic Boundary Conditions (PBC). The system is placed in a unit cell, usually a cubic or truncated octahedral unit cell. The main unit cell is then surrounded by identical images. The long-distance non-bonded interaction energies are calculated between the unit cell and the images that are within the 
cutoff distance. Each atom in the main unit cell has identical images surrounding the unit cell that follows the trajectory of the main unit cell particle. During the course of the MD simulation, if for example, a water molecule from the main unit cell moves out of the unit cell an identical image water molecule enters the main unit cell. This image water molecule then becomes part of the main unit cell when the atom list is updated. Therefore, $\mathrm{PBC}$ conserves mass, the number of particles and total energy. An important consideration is the size of the main unit cell. Ideally, the size of the main unit cell should be the total length of the molecule being modeled plus two times the largest cutoff distance used for non-bonded interactions. When the size of the unit cell is defined in this way, no two solute atoms will interact with each other directly and no solvent molecule will see two copies of a given solute atom.

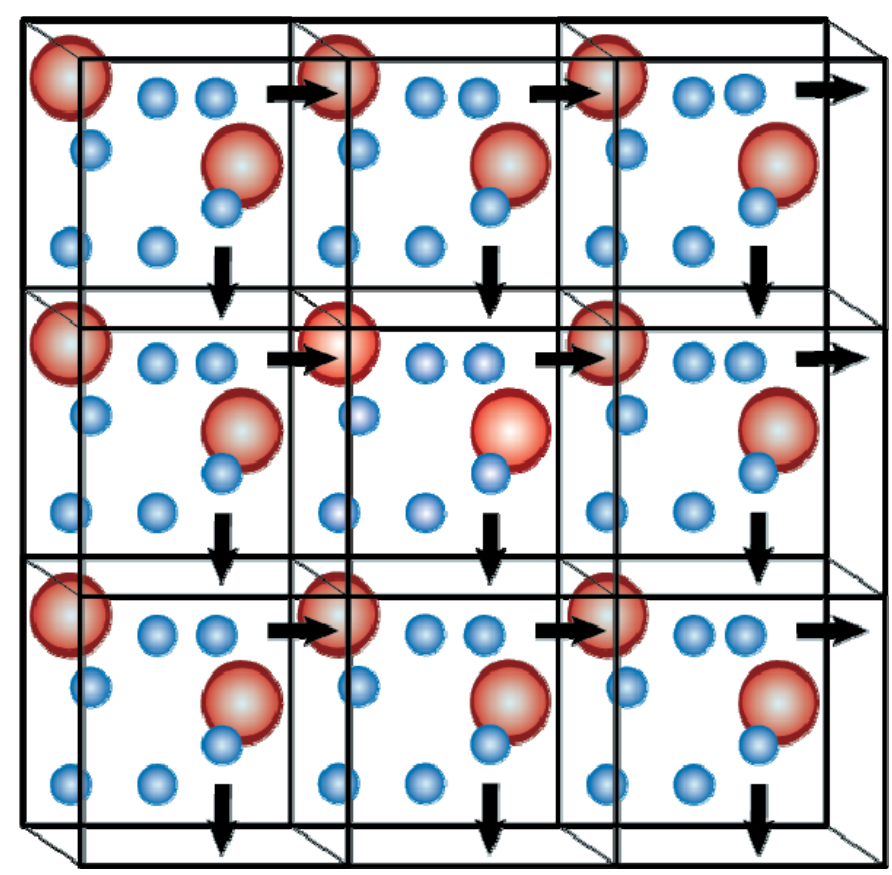

Figure 17: Schematic representation of periodic boundary condition 


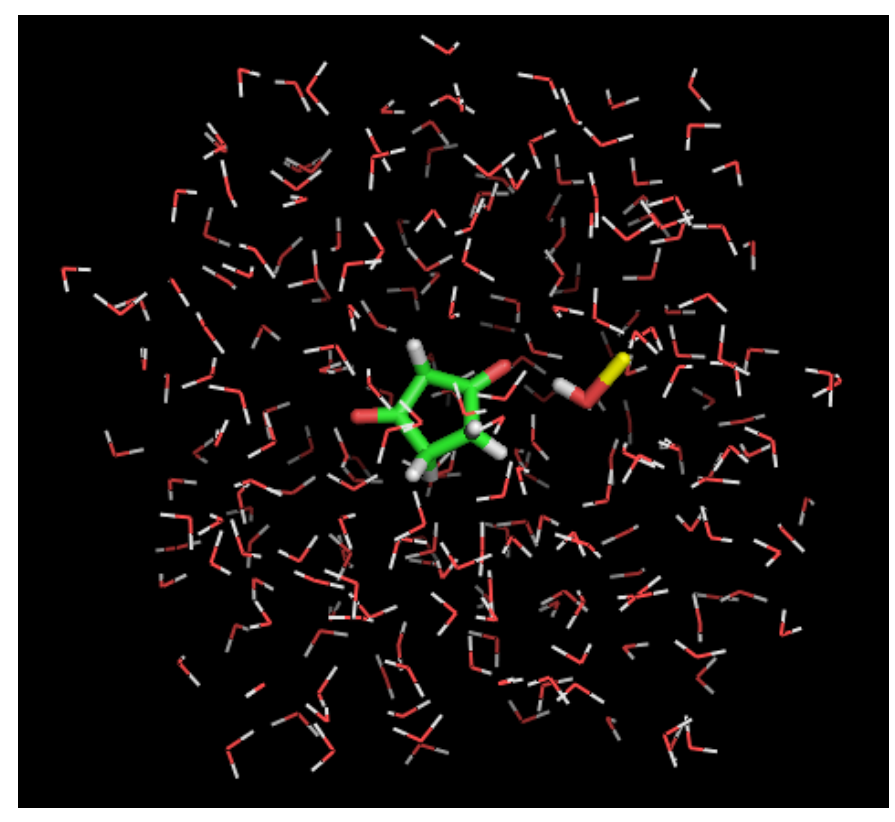

Figure 18: Solvated CPD and HOCl in a truncated octahedron box of water

\subsection{Quantum Mechanics}

To study reactions that involve the formation of new bonds or breaking of bonds, one needs to solve the equations of Quantum Mechanics (QM) applied to the electronic structure of molecules. The QM model is described by the time-independent Schrödinger equation:

$$
\hat{H} \psi=E \psi
$$

where $\psi$ is the wavefunction describing the system, $\hat{H}$ is the Hamiltonian operator and $E$ is the total energy of the system. The Hamiltonian operator for the time-independent Schrödinger equation is given by the following: 


$$
\hat{H}=-\sum_{i} \frac{\hbar^{2}}{2 m_{e}} \nabla_{i}^{2}-\sum_{k} \frac{\hbar^{2}}{2 m_{k}} \nabla_{k}^{2}-\sum_{i} \sum_{k} \frac{e^{2} Z_{k}}{r_{i k}} \nabla_{i}^{2}+\sum_{i<j} \frac{e^{e}}{r_{i j}}+\sum_{k<l} \frac{e^{e} Z_{k} Z_{l}}{r_{i l}}
$$

where $i$ and $j$ label electrons, and $k$ and $l$ label nuclei. The first two terms describe the kinetic energy of electrons and nuclei, respectively, and the last three terms describe the potential energy of interaction electrons with nuclei, of electrons with each other, and of nuclei with each other, respectively. To simplify the above Hamiltonian the BornOppenheimer approximation is applied, which decouples the correlated motion of electrons with nuclei. The nuclei are assumed fixed in space while the energy of the electrons surrounding the nuclei is calculated. The above Hamiltonian is then simplified to the following (nuclear-nuclear repulsion energy can be added back in after the electronic energy is calculated):

$$
\hat{H}=-\sum_{i} \frac{\hbar^{2}}{2 m_{e}} \nabla_{i}^{2}-\sum_{i} \sum_{k} \frac{e^{2} Z_{k}}{r_{i k}} \nabla_{i}^{2}+\sum_{i<j} \frac{e^{e}}{r_{i j}}
$$

Most approaches to solving the Schrödinger equation begin by expressing the wavefunction as a sum over basis functions:

$$
\psi=\sum_{i=1}^{N} a_{i} \phi_{i}
$$


where $\phi_{i}$ is a basis function and $a_{i}$ is a coefficient. When the basis functions are appropriately chosen and spin is correctly accounted for, the correct solution for $\psi$ can in principle be determined with an appropriate methodology.

\subsubsection{Theoretical Methods}

It is not practical to solve the Schrödinger equation exactly for any but the simplest of systems which include only one electron, for example a hydrogen molecule ion, because of the electron-electron repulsion term. Hence, there are several approximations made to solve systems of interest. First, the basis set expansion above must be truncated at a finite number of orbitals. In most approaches, the basis functions in the expansion above are themselves expanded further:

$$
\phi_{i}=\sum_{j=1}^{M} a_{j} \varphi_{j}
$$

The functions $\varphi_{j}$ are termed atomic orbitals, and the functions $\phi_{i}$ are termed molecular orbitals. When either expansion is finite (which must be the case for practical applications), the solution will in principle be approximate. A molecular orbital constructed as a linear combination of atomic orbitals is called a linear combination of atomic orbitals (LCAO).

One of the most basic methods for solving the Schrödinger equation, the Hartree-Fock (HF) method, makes the approximation that each electron is influenced by an average 
static potential of the remaining electrons in the system and an iterative 'self-consistent field' (SCF) method is used to determine the wavefunction. The HF molecular orbitals consist of Slater Determinant wavefunctions that include the exchange effects of the electrons. However, the HF method neglects most aspects of electron correlation, and this approximation may yield large errors in properties calculated from the wavefunctions. More sophisticated and robust methods need to be used, but HF often provides a practical stepping stone to a more exact solution.

There are a large number of methods for including more aspects of electron correlation than HF theory does. Some of the most widely used methods are MollerPlesset (MP $n$ ) perturbation theory and Coupled Cluster (CC) theory. The MP $n$ method depends on the perturbation order $(n)$ correction used. However, these methods do not scale too well with the number of basis set and are computationally very expensive. For example, the CCSD (CC with single and double excitations) scales at the order of $\mathrm{N}^{6}$ and MP2 (with second-order perturbation correction) scales at the order of $\mathrm{N}^{5}$, where $\mathrm{N}$ is the number of basis sets. All of the methods described thus far are called ab initio methods because they do not make reference to any empirical parameters, and thus construct a solution from first principles.

Another alternative method is Density Functional Theory (DFT), which is different from the wavefunction theory discussed so far. The DFT approach accounts for electron correlation and offers equal or comparable accuracy to MP2 methods with much better computational efficiency. Scaling of DFT is of the order $\mathrm{N}^{3}$, but for practical purposes (discussed later in this section) it scales to the order of $\mathrm{N}^{4}$. Density Functional 
Theory scales substantially better than the other methods., For these reasons, I use DFT for my calculations.

Density functional theory was first introduced in 1927 by Thomas and Fermi, but the approximations made were inaccurate. The theory was later legitimatized by the proof of two theorems, the Hohenberg-Kohn existence theorem and the Hohenberg-Kohn variational theorem in 1964. The most difficult part of DFT is not having an analytical form for the exchange correlation energy, and for this reason some approximations are made. One is to use parameters that are empirically optimized. Hence, DFT is sometimes referred to as a semi-empirical method, but in fact the functionals used are not parameterized to the extent that semi-empirical molecular orbital theory is. Density functional theory makes an approximation in order to calculate the energy density, a component of the exchange correlation energy. One form of the approximation, called local density approximation (LDA), treats the electron density as a uniform electron gas and is single valued at every position. Because of this approximation we can expect limitations in accuracy. One way to improve the exchange correlation functional is to allow it to depend not only on the local electron density but also on the extent on the gradient of the local density. This approximation is called the generalized gradient approximation (GGA).

The most popular GGA exchange functional is due to Becke (B); it incorporates a single empirical parameter. The most popular GGA correlation functional is LYP. The functional I use, B3LYP, is a hybrid functional that uses B3 with a three parameter scheme for the exchange functional and an adiabatic connection method (ACM) and LYP 
for the correlation functional. ACM methods are called hybrid methods because they incorporate HF and DFT exchange terms.

I chose the B3LYP/6-31+G** level of theory and basis set because it has been shown to be sufficiently accurate for cases like my system, and it is sufficiently fast. The software packages I used for the QM calculations were Gaussian98 and Gaussian09 [56].

\subsubsection{Basis sets}

Choosing an appropriate basis set is very important for achieving an accurate solution to the electronic structure problem. In practice, the atomic orbitals in the second expansion above are themselves further expanded in terms of other functions. I will briefly discuss these. It is computationally efficient to represent atomic orbitals in terms of Gaussian type orbitals (GTOs) [46]. While GTOs are computationally convenient, they have issues with representing the radial portion of the orbital. The best way to deal with this drawback of GTOs is to form linear combination of GTOs. Basis sets are made up of linear combinations of primitive GTOs called contracted orbitals. Using primitive and contracted orbitals gives more flexibility in manipulating the coefficients so as to have a proper radial representation of the orbital. It is found that the most efficient (accuracy vs. computational efficiency) way to create a minimally realistic representation of an atomic orbital is in terms of three contracted orbitals. The nomenclature used to describe this kind of representation is "STO-3G basis set." 
For the accuracy that I need, larger basis sets than the STO-3G are needed. The most important considerations for increasing the basis set size are described next. It is known that the valence orbitals play a much more major role in reactions than the core orbitals. Hence, there is more to be gained by having flexibility in the valence basis functions than in the core basis functions. This led to the concept of a 'split-valence' basis set, in which the core orbitals are represented by a single contracted basis function and the valence orbitals are represented by several contracted basis functions. I use the 631G basis set in my QM calculations. The ' 6 ' in the basis set representation indicates that the core orbitals are represented by one contracted orbital consisting of six primitive orbitals. The other two numbers, ' 31 ', indicate that the valence orbitals are represented by two contracted basis functions each. The first contracted basis function includes three primitive functions and the second contracted function includes one normalized primitive function. In addition polarization and diffuse functions can be augmented to the basis set, which are described below.

\section{Polarization functions}

Several molecular properties and especially geometries are sensitive to polarization effects. Atomic orbitals can be accurately represented with $\mathrm{s}$, $\mathrm{p}$ functions, etc. This is because atomic orbitals, like these functions, are centered about the atom nucleus. Molecular orbitals, on the other hand, are often extended in space and are expanded in terms of LCAOs with several atom nuclei. For this reason it is necessary for the molecular orbital to have more flexibility than atomic orbitals. 
One way to provide this flexibility is to include an additional quantum number of higher angular momentum than the valence orbitals in the form of a GTO basis function. This means that elements in row two like carbon and oxygen. have polarization functions that include d-type GTOs; for hydrogen atoms the polarization functions includes p-type GTOs. The nomenclature used in this case is either a '*' or, equivalently, (d) at the end of the basis set specification for row two heavy atoms; and '**' or, equivalently, $(\mathrm{d}, \mathrm{p})$ when polarization functions are to be used for both second row atoms and hydrogen atoms. In my calculations I use the following basis set specification and polarization functions: 6$31 \mathrm{G}^{* *}$.

\section{Diffuse functions}

Electrons in some molecular complexes tend to be more spatially diffuse than in atomic orbitals. To provide the flexibility needed to represent this spatial diffuseness, the basis function is augmented with diffuse functions when needed. The nomenclature used to indicate the addition of diffuse functions is a '+' added to the basis set specification for additional heavy atom $\mathrm{s}$ and $\mathrm{p}$ functions and a ' ++ ' to indicate diffusion functions of $\mathrm{s}$ type for hydrogen atoms in addition to heavy atom diffuse functions. In my calculations I use diffuse function for only heavy atoms; $6-31+\mathrm{G}^{* *}$. 


\subsection{Quantum Mechanics / Molecular Mechanics}

As described in previous chapters MM is useful for modeling large systems. However, it cannot be used to model reactions that involve bond-making or bondforming. Instead we use a QM model to model chemical reactions. The disadvantage to the QM model is that it does not scale well as the number of heavy atoms increases and therefore the limiting factor is the size of the system. We are restricted to using the QM model for only small systems, or for reactions of large systems, one has to ignore atoms not near the reaction site. In many cases, though, these atoms may significantly influence the reaction even though their role may not be as important as the ones near the reaction site. These atoms may influence the reaction via long range non-bonded interactions (electrostatic or van der Waals) with the reactive atoms or, in the case of macromolecules, by influencing the conformation of the system as a whole.

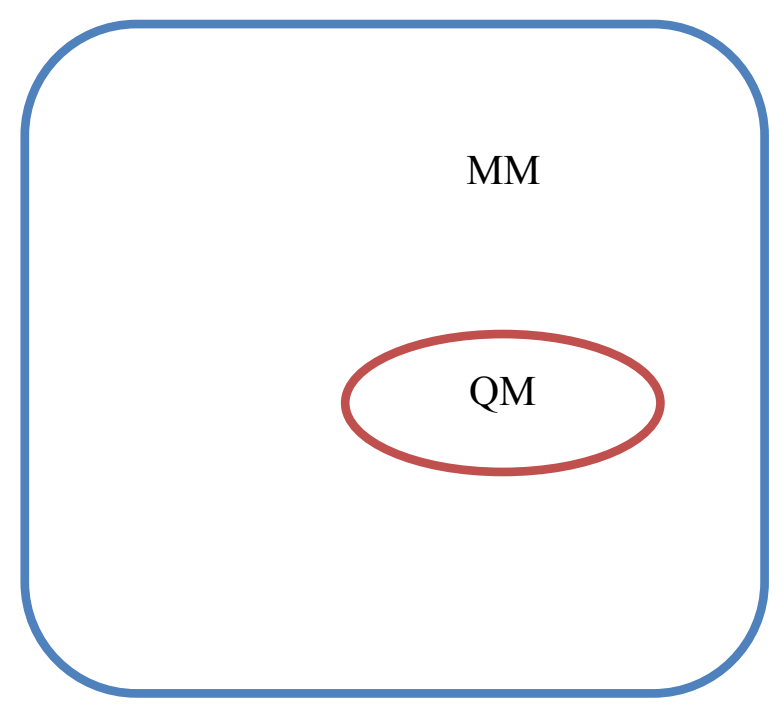

Figure 19: Schematic of $Q M$ and $M M$ regions 
Fortunately, larger systems can often be treated by using a combination of the MM and QM approaches. This kind of a hybrid method is denoted QM/MM. One can model reactive site atoms that are deemed crucial to the reaction using QM. The other atoms of the large system can then be modeled using MM instead of ignoring them. The development and application of QM/MM methods have grown rapidly over the past few years.

For a $\mathrm{QM} / \mathrm{MM}$ model, the total Hamiltonian is written as

$$
H_{\text {Total }}=H_{Q M}+H_{M M}+H_{Q M / M M}
$$

This equation has a deceptively simple appearance. The details of $\mathrm{H}_{\mathrm{QM} / \mathrm{MM}}$ can become quite complicated, depending on the system. The range of factors that can arise is described below, but as in earlier chapters emphasis is given to factors arising in the research reported here.

The first thing that comes to mind when we consider QM/MM systems is the boundary between the QM section and MM section and whether the boundary intersects a bond or not. It is a little simpler if the boundary does not intersect a bond. If it does, there are a few ways it is handled. One way is to use a link atom, usually hydrogen, to cap off the dangling bond in the QM section. Another way is to use a frozen orbital. Sometimes a different methodology, ONIOMM, is used. In my system, the boundary does not intersect any bond, so I did not have to deal with any of these issues.

Even though my system does not have to deal with the issue of the QM/MM boundary severing bonds, there are still other matters that need to be considered and 
choices to be made. One is the coupling or polarization between the two regions. There are three possible interactions. Both regions can be unpolarized, only the QM region is polarized and the third option is having both regions polarized.

My model, incorporates polarization of the QM region by the MM region, but not polarization of the MM region. The MM atoms are represented as fixed point charges. The Hamiltonian for such a system is

$$
H_{Q M / M M}=\sum_{i}^{\text {solute electrons }} \sum_{m}^{M M \text { atoms }} \frac{q_{m}}{r_{i m}}+\sum_{k}^{\text {solute nuclei }} \sum_{m}^{M M \text { atoms }}\left[\frac{Z_{k} q_{m}}{r_{k m}}+4 \varepsilon_{k m}\left(\frac{\sigma_{k m}^{12}}{r_{k m}^{12}}-\frac{\sigma_{k m}^{6}}{r_{k m}^{6}}\right)\right]
$$

Another issue that needs to be considered is the interaction of atoms in both regions that are very close to the boundary. MM model represents atoms as point charges, while the QM atoms have electronic and nuclear representation of atoms. The MM point charges near the boundary can have large unrealistic influences on the electronic representation of the QM atoms near the boundary. They can distort the QM electron density and create forces acting on QM nuclei. One way to deal with this situation is to blur the point charges in the MM region that are close to the boundary by Gaussian functions so that the charges are spread or 'blurred' and do not have such drastic, unphysical effects on QM atoms close to the boundary. The CHARMM program includes such an option, and I used it in the work reported here.

The QM software I use to interface with CHARMM to implement the hybrid QM/MM model is Q-Chem 3.2 [57]. 


\subsection{Chain-of-replica Methods}

A reaction mechanism is better understood by studying its reaction path. A reaction path is the relative motion taken by atoms during a chemical reaction. We usually think of a reaction path as beginning with reactants, proceeding through a highenergy structure called a transition state, and terminating in products. In complicated cases, there may also be significant intervening structures called intermediates. For any chemical reaction, there are many possible reaction paths. Particular significance is given to that reaction path that passes through the lowest-energy transition state and for which the path from the transition state toward either reactants or products follows the direction of steepest descent; this is called the Minimum Energy Path (MEP) or Steepest Descent (SD) path. The significance of the MEP is that in a macroscopic system of many reacting molecules, the vast majority of reactive events follow a trajectory close to the MEP, and consequently the energetics of the MEP dominates the kinetics. Thus the MEP is often predictive of rate constants and reaction mechanisms, and so most analyses are based on the MEP. In mathematical terms, reactants, products and transition states (TS), and also intermediates if they exist, are stationary points because for them the gradient of the potential energy surface is zero. Clearly, the development of methods to identify MEPs, and especially the stationary points, is very important in understanding reaction mechanisms.

There are broadly three classifications of methods used to identify the reaction path. The first are eigenvector following methods, of which the most popular method is the Intrinsic Reaction Coordinate (IRC) method [58, 59]. This method relies on first 
identifying the transition state, for which by definition the Hessian possesses exactly one negative eigenvalue, and then following the eigenvector corresponding to the negative eigenvalue in the forward and reverse directions. This process eventually leads to the reactant and product structures. While it is robust, there are several drawbacks to this method. It is sometimes very difficult to find the TS structure if the potential energy surface is complex or the saddle point is quite flat. Locating the TS is also a very expensive method in terms of scaling. Memory scales as $\mathrm{N}^{2}$ and time scales as $\mathrm{N}^{3}$, where $\mathrm{N}$ is the number of degrees of freedom. The size of the system restricts the use of this method. It is not practical to use in large systems such as the one studied here.

Another method which is quite intuitive is the reaction coordinate following method. If we know the reaction coordinate that drives a particular reaction, we just follow it and it should connect the three stationary points. The reaction coordinate following method, however, is impractical for all but the simplest of systems, since it is very difficult to know the reaction coordinate in advance, especially for reaction mechanisms that involve large numbers of atoms. Another issue with this method is that one needs to check the hysteresis to make certain that the same path is mapped out in the forward and reverse directions.

The method I use is from a group of methods called chain-of-replica methods [6071] that are fairly new compared to the other methods. Its development is quite significant because it allows one to study reaction mechanisms of large systems, and in most cases only first derivatives need to be calculated. In some cases the results can be refined using a second derivative method for geometry optimization, but even then, chain-of-replica methods are more feasible for large systems than are other methods. 
Another significant advantage of chain-of-replica methods is that one only needs the reactant and product structures as input, whereas the IRC method also requires a fully optimized transition state. In fact, chain-of-replica methods are efficient for locating transition states in high-dimensional systems where the usual transition-state geometry optimization methods may be impractical. There is also no need to know the reaction coordinate in advance which can be a very difficult task with a system having many degrees of freedom. A further benefit of this method is that it can take advantage of today's parallel computers. Since individual points on the path are only weakly coupled, they can be refined on different processors. The other methods are sequential and cannot make use of parallel computers as efficiently.

The chain-of-replica method was originally proposed by Elber and Karplus [67]. It was based on work by Empedocles who in 1969 had proposed using a line integral for searching a reaction coordinate.

$$
S=\frac{1}{L} \int_{R_{A}}^{R_{B}} V(R) d l(R)
$$

where $S$ is the function to be optimized, $R_{A}$ and $R_{B}$ are position vectors representing the coordinates of reactant and product structures, $\mathrm{V}(\mathrm{R})$ is the potential energy and $\mathrm{dl}(\mathrm{R})$ is the line element along the length of the path, $L$, between $R_{A}$ and $R_{B} . V(R)$ and $d l(R)$ are functions of the $\mathrm{N}$-dimensional position vector representing coordinates, where $\mathrm{N}$ is the number of degrees of freedom.

Elber and Karplus approximated the above line integral by a discretized version. 


$$
S=\frac{1}{L} \sum_{j=0}^{M} V\left(R_{j}\right) \Delta l_{j}+\text { constraints }
$$

where $\mathrm{M}$ is the number of intermediate discretized grid points and $\Delta l_{j}$ is the interval between each point. Constraints were included to keep the individual points equidistant from each other. This is because, during the process of minimization, if the $\Delta l_{j}$ were widely different the equation above would no longer be a good approximation of the line integral. Later additional repulsion constraints were added so that the points did not all pool into a local minimum. In the methods I used that are described below, the distance $\Delta \mathrm{l}$ between coordinate sets representing points on the path is defined as the root mean square difference (RMSD) between the atomic coordinates of the two sets. The definition of $\Delta \mathrm{l}$ includes overall translation and rotation of the coordinate sets to achieve a best fit in a least squares sense. The total length of the path, $L$, is the sum of the distances $\Delta 1$ between points on the path so defined. Thus, L is a function defined in RMSD space, $\mathrm{L}\left(\mathrm{r}_{1}, \mathrm{r}_{2}, \ldots \ldots, \mathrm{r}_{\mathrm{M}-1}\right)$, where $\mathrm{r}_{\mathrm{i}}$ is an RMSD of coordinates just defined..

The formalism described above forms the basis for all chain-of-replica methods. Individual methods differ mainly in the way the points are generated, the minimization protocol used, and most importantly the kind of constraints imposed to control the optimization.

The methods I used for include the Replica Path method and the Nudged Elastic Band (NEB) method with the Climbing Image algorithm to obtain the TS structure (CINEB). I also used the Adaptive Nudged Elastic Band (ANEB) method for a single test 
case. All these methods used are implemented in CHARMM, the software I used for my calculations.

\subsubsection{Replica Path Method}

The easiest and most common way to create initial replicas or images is by linear interpolation of the reactant and product structures. The replica path method, like most modern chain-of replica methods, only replicates certain atoms that are deemed important in the reaction being studied instead of the entire system. The un-replicated part of the system is still present and identical across all replicas. The un-replicated part can and will affect the replicas and respond, if needed, but it will do so the same way for all replicas along the MEP. This, quite conveniently, sets up for use with the QM/MM model. It allows the focus of the study and resources on the active site of the molecule. In the geometry optimization calculation of the replicas the un-replicated atoms were kept fixed.

The main aspect of replica path that sets it apart from the other chain-of-replica methods is that the method uses a harmonic penalty function to constrain/restrain the points on the reaction path. The penalty function applied is directly proportional to the root mean square deviation (RMSD) in the atomic coordinates of two neighboring replicas or images. During the evaluation of the RMSD the deviation because of rotation and translation are avoided. An advantage of using this RMSD space to define the restraints is that there is no need to deal with effects of rigid body rotation and translation which would otherwise affect the minimization process. 
Below are the penalty functions and other keywords used with replica path as implemented in CHARMM.

$$
E_{r m s}=\frac{1}{2} \sum_{i=1}^{N} K_{r m s}\left(r_{i}-\bar{r}\right)^{2}
$$

where $E_{r m s}$ is the penalty function used to force the replicas or images to be approximately equidistant from each other. $\mathrm{N}$ is the number of replicas or images along the pathway. The optimal value of the force constant $K_{r m s}$ should result in a path step variation of no greater than $15-20 \%$. The distance between path points $i$ and $i+1$ in RMSD-space is denoted $r_{i}$, and the average over all pairs of consecutive path points is $\bar{r}:$

$$
\begin{gathered}
r_{i}=R M S D_{\text {best fit }}(i, i+1) \\
\bar{r}=\sum_{i=1}^{N} \frac{r_{i}}{N}
\end{gathered}
$$

One artifactual difficulty sometimes encountered is a tendency for the reaction path to become highly curved so that points fall into each other, resulting in small values of the constraint energy but giving unrealistic paths. To avoid this problem, an additional restraint, $E_{\text {angle }}$, is used to keep the reaction path realistically linear: 


$$
\begin{array}{ll}
E_{\text {angle }}=\frac{1}{2} \sum_{i=1}^{N} K_{\text {angle }}\left[\operatorname{COSMAX}-\cos (\Theta)_{i}\right]^{2} & \text { if } \operatorname{COSMAX}>\cos (\Theta)_{i} \\
E_{\text {angle }}=0 & \text { if } \operatorname{COSMAX} \leq \cos (\Theta)_{i}
\end{array}
$$

where $\Theta$ is the $(i-1)-i-(i+1)$ angle in RMSD-space. COSMAX is a value between 0.9 and 0.98 , with the value 0.98 making the pathway more linear and rigid. I use the value 0.9 in my calculations. $K_{\text {angle }}$ is the force constant used for the restraint. The recommended range is $100-500 \mathrm{kcal} / \mathrm{mol}$. I used the value $100 \mathrm{kcal} / \mathrm{mol}$ in the calculations reported here. Below are the definitions of the most important keywords used in the CHARMM's replica path facility.

RMAX: Maximum distance the replicas are allowed to be separated before switching on the force constant KMAX instead of $K_{r m s}$ in the penalty function $E_{r m s}$.

KMAX: The force constant to be used when the distance between replicas exceeds RMAX and is usually greater than or equal to $K_{r m s}$.

WEIGHT: This keyword causes the values in the main weighting array are to be used. This allows one to place more emphasis on atoms that play a major role in the reaction, so that when the RMSD is calculated to constraint the replicas more emphasis is placed on those atoms. 
ROTATION and TRANSLATION: Keywords that turn on the RMSD best-fit procedure between neighboring replicas, thus preventing the rigid-body rotations and translations from effecting the minimization. Without the RMSD best-fit procedure the atoms would adjust unnecessarily by rotating and/or translating in order to meet the constraint requirement.

BLOCK: The utility in CHARMM that calculates the total potential energy at each point along the minimum energy path. The total potential energy is the sum of the interaction energy between atoms in the un-replicated region plus the interaction energy between atoms in an individual replica plus the interaction energy between atoms in a single replica and the atoms in the un-replicated region. The BLOCK utility has to be appropriately setup so that the inter-replica interactions are ignored and the interaction matrix is appropriately scaled. The scaling factor for the interaction energy between atoms in the un-replicated region is set at 1.0. The scaling factor for the interaction energy between atoms in an individual replica is the inverse of the number of replicas created. The scaling factor for the interaction energy between atoms in a single replica and the atoms in the un-replicated region is the inverse of the number of replicas created.

\subsubsection{Nudged Elastic Band Method}

The NEB method is similar to the replica path method and uses the same keywords in CHARMM. The implementation, however, is different. The NEB method 
was originally proposed to address a concern about replica path. In replica path, the physical interatomic interactions and the extra spring restraints can interact, potentially causing deviations from the true MEP. Such deviations are especially likely to occur in steep regions of the reaction path, such as are often encountered near a transition state. While replica path method implements the penalty function based on the RMSD as described above, NEB uses a force projection scheme in which the physical force due to interatomic interactions acts only perpendicular to the path, and spring-constraint forces act only along the path. The spring forces keep the replicas separated from each other and the potential forces acting perpendicular to the path 'nudge' the pathway closer to the MEP. This is accomplished with the following set of equations.

$$
F_{i}^{N E B}=F_{i}^{\perp}+F_{i}^{S \|}
$$

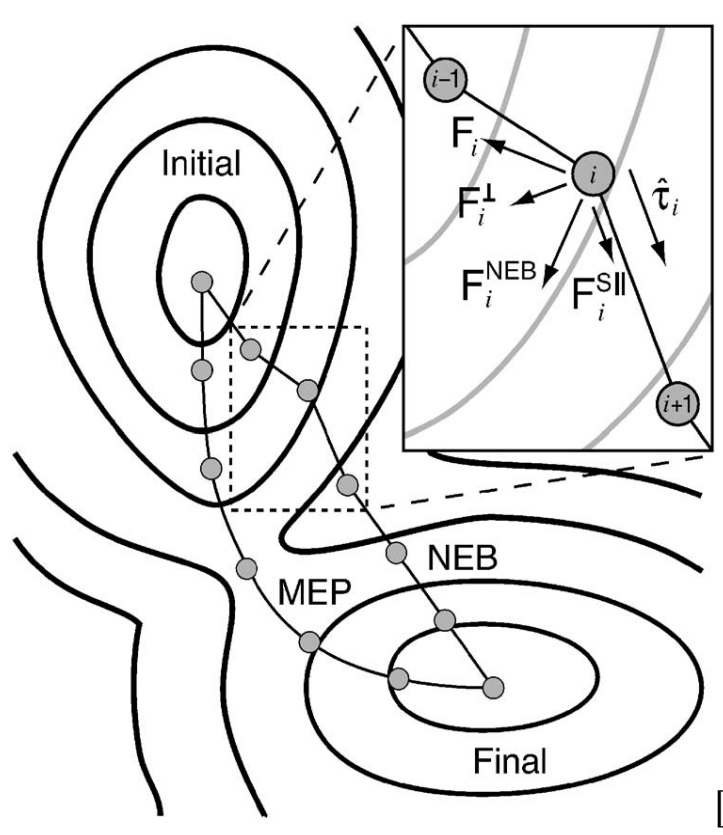

Figure 20: NEB 
where $F_{i}^{\perp}$ is the component of the force due to the potential perpendicular to the band given by

$$
F_{i}^{\perp}=-\nabla\left(R_{i}\right)+\nabla\left(R_{i}\right) \bullet \hat{\tau}_{i} \hat{\tau}_{i}
$$

where $\hat{\tau}_{i}$ is the unit vector in the tangential direction of the higher energy neighboring replica, and $F_{i}^{S \|}$ is the spring force parallel to the band and is given by

$$
F_{i}^{S \|}=k\left(\left|R_{i+1}-R_{i}\right|-\left|R_{i}-R_{i-1}\right|\right) \hat{\tau}_{i}
$$

\subsubsection{Climbing Image - Nudged Elastic Band Method}

A problem often encountered in the methods described above is that the actual transition state may be difficult to reach, and the resolution of replicas near the transitions state may be poor. Poor resolution is particularly problematical if the energy barrier is narrow in comparison to the length of the minimum energy path. What is usually noticed in such situations is that the replicas tend to slide away from the transition state and cause 'corner-cutting' around the transition state.

The climbing image algorithm (CI-NEB) is a modification of the NEB method. The main difference is the treatment of the replica that has the highest energy in the pathway after a few minimization steps. That point is not subjected to the spring forces 
and is driven towards the transition state instead of being restricted by the spring forces. The total force acting on that particular point is given by

$$
\begin{gathered}
F_{i_{\max }}=-\nabla E\left(R_{i_{\max }}\right)+\left.2 \nabla E\left(R_{i_{\text {max }}}\right)\right|_{\|} \\
F_{i_{\max }}=-\nabla E\left(R_{i_{\max }}\right)+2 \nabla E\left(R_{i_{\max }}\right) \bullet \hat{\tau}_{i_{\max }} \hat{i}_{i_{\max }}
\end{gathered}
$$

This modification to the NEB does not add any additional computational time. The replica moves along the elastic band up the potential energy surface to the transition state.

\subsubsection{Adaptive Nudged Elastic Band Method}

The ANEB method [61] is a variation of the NEB method. The traditional NEB method can sometimes have difficulty approaching the transition state structure due to poor convergence on difficult and flat potential energy surfaces. The focus of the ANEB method is to increase the resolution of points or replicas near the transition state and to acquire a good estimate of the reaction barrier. Just like the regular NEB method the initial setup requires two local minima representing the reactant and product structures. The initial replicas or images may be created by linear interpolation as in the NEB method and minimized until a reasonable convergence criterion is met. At this point ANEB deviates from the regular NEB method. New fixed points are selected instead of the reactant and product structures in the previous step. The new fixed points selected are 
the ones on either side of the point or replica with the maximum energy. Presumably, these points encompass the actual transition state. New images are then created between these points as in regular NEB and minimized. This procedure is continued until the transition state is found. Hence, the resolution of points or replicas is adaptively increased near the transition state. Another benefit of the ANEB method is that fewer replicas are needed at each iteration.

(a)
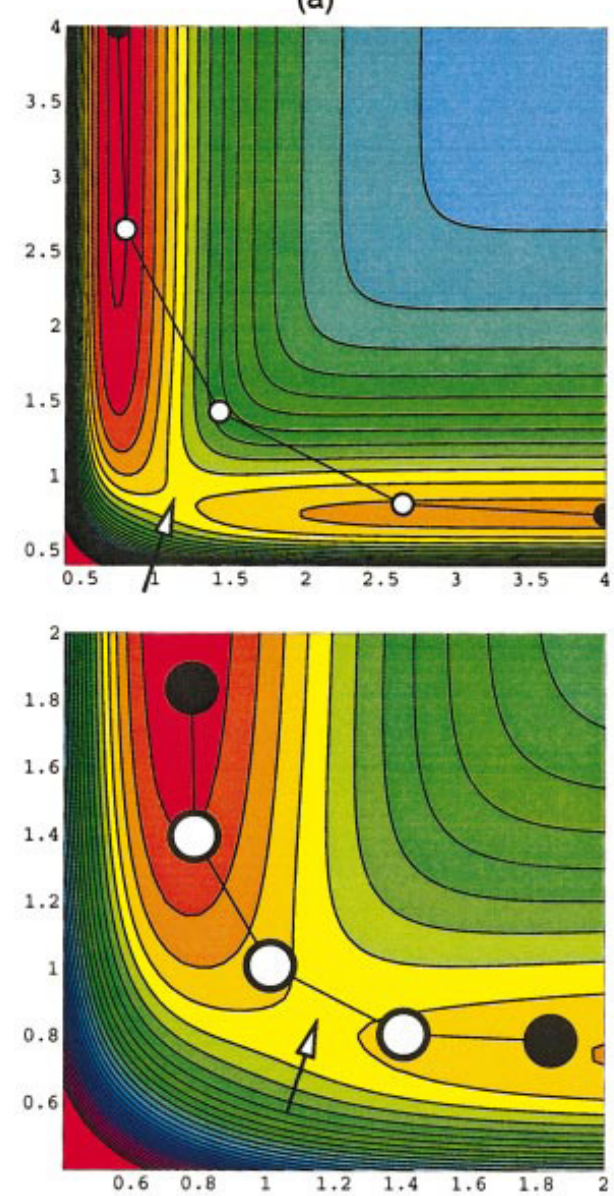

(c)
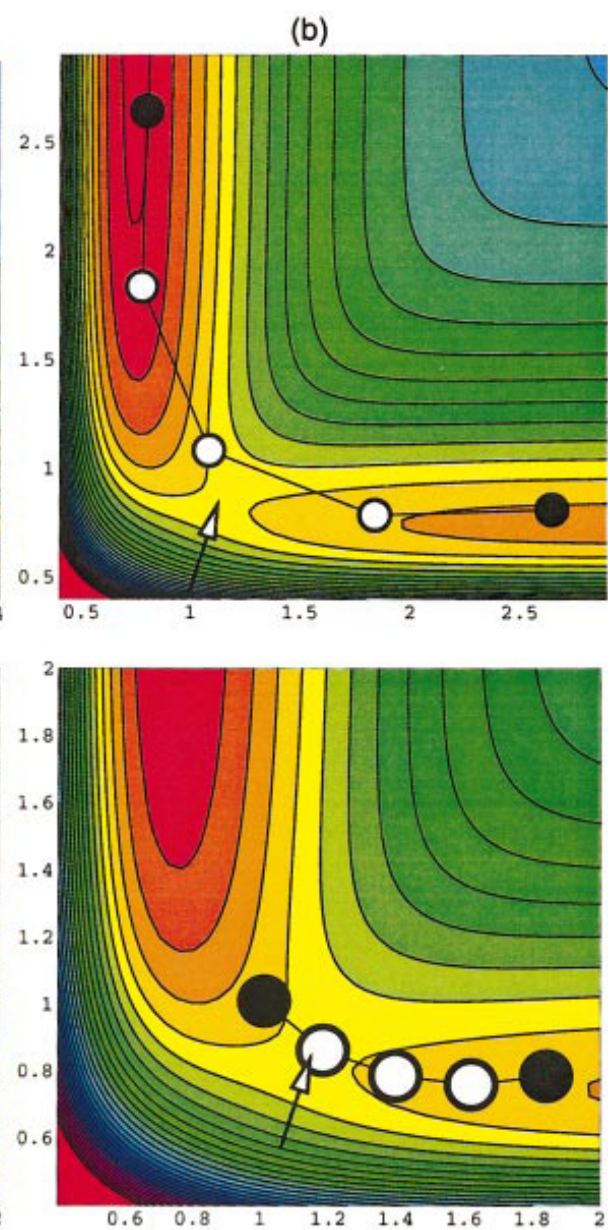

(d)

Figure 21: ANEB 


\section{PARAMETERIZATION}

\subsection{Principles}

As discussed earlier in the Molecular Dynamics and QM/MM sections, programs running MD calculations require parameters that need to be read at the beginning of the script. The default parameters provided by the software are adequate for most systems or molecules. However, in some rare cases one has to develop one's own set of parameters if the software does not provide parameters for the molecule being studied.

Parameters are provided in two files called the topology file and the parameter file. The topology file contains information such as the names of molecules, atom names and atom types (defined below) partial charges on individual atoms, atom connectivity (bonds), and if present, improper dihedral angles, acceptor or donor atoms, internal coordinates and patch information (primarily need for protein molecules). The molecule name is used by the script to build or make reference to the molecule and hence has to be unique with a maximum of four characters. The connectivity information lets the software know which atoms are connected and if they share a single, double or triple bond. Acceptor or donor atom information is used for hydrogen bond analysis. A data structure for internal coordinates is set up to reserve memory in case internal coordinate manipulations are needed or in order to build Cartesian coordinates from internal coordinates. Patch information is used to connect fragments or segments and in some cases it tells the program how to handle a residue at the ends of a segment. 
The parameter file contains information such as the atom type, Van der Waals constants, force constants for bonds, angles, dihedrals and impropers and their respective equilibrium values. This information is used in energy calculations as described earlier. In cases where there are no parameters available for the system or molecule(s) being studied one has to develop one's own parameters. Depending on the software being used there are different protocols. One cannot mix and match parameters from different types of software. For example, one cannot use some parameters from Amber and some from CHARMM. All the parameters have to be from either Amber or all from CHARMM for internal consistency. Hence, it is very important to be consistent in using the correct protocol. Alexander MacKerell has become the primary developer of parameters for the CHARMM suite of force fields. I use the CHARMM parameter set and therefore follow the protocol outlined by MacKerell et al. to develop the parameters for my system [72].

There are two ways to go about developing parameters for atoms or molecules for use in CHARMM. The first and simplest way is called "by analogy." One identifies fragments of the molecule being parameterized that are similar to fragments of molecules already present in the default CHARMM parameter files. If it is possible to identify a complete set of fragments that when combined yield the complete molecule, only a few parameters need to be developed. These are for connecting the individual fragments. The analogy method cannot be used if the default parameter set lacks the needed fragments. In that case, one must develop all parameters for the entire molecule from the ground up. If the parameter set contains only a few of the needed fragments, it is generally observed that developing all parameters from the ground up yields the best parameterization. 
Below is a flowchart showing the process by which parameters are developed.

Following it, the procedures used to develop parameters for the systems of interest to us are described in detail.

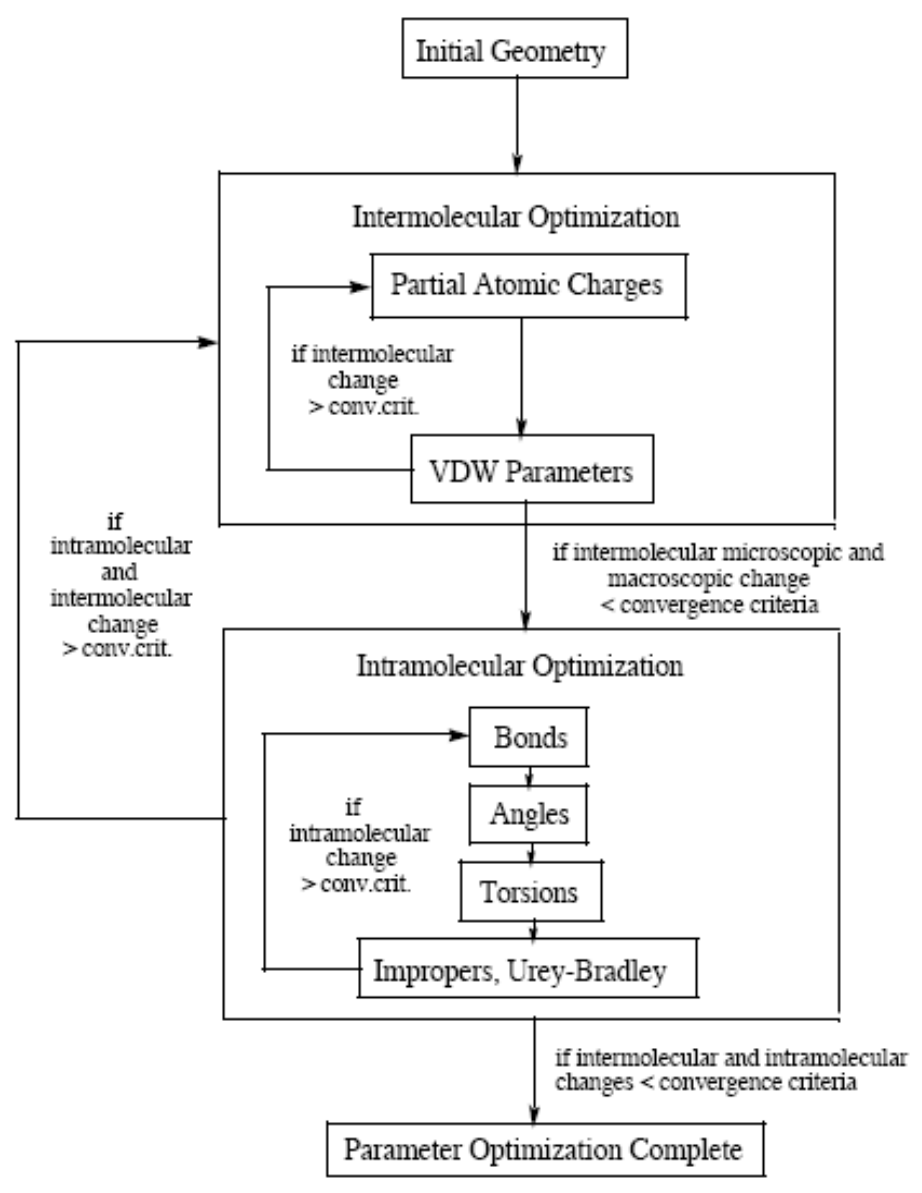

Figure 22: Parameterization protocol flow chart 


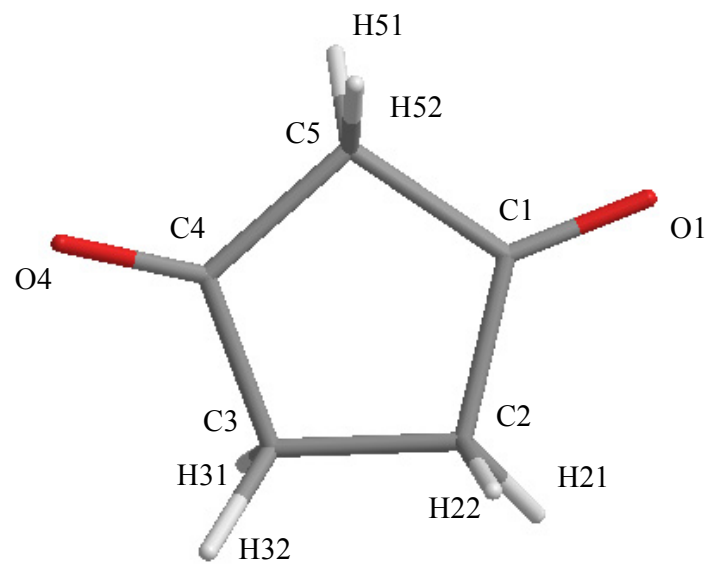

Figure 23: Cyclopentanedione (CPD). Atom names are shown

The molecule CPD is directly involved in the chlorination reaction being studied. Therefore highly accurate parameters are needed and I choose to develop all of them from the ground up. I only need to develop parameters for the diketo form of CPD and not the ketoenol form, since I only need the parameters to perform MD simulations involving the diketo form to identify "unique encounter reaction precursors," as explained in chapter 5. I will actually begin with a set of parameters determined by analogy, but I subsequently refine them. First, though, I must define a few terms.

\subsubsection{Atom type and atom name}

In Figure 23, CPD is shown with a unique "atom name" for each atom. In force fields, in addition to a unique "atom name," each atom is also assigned an "atom type." "Atom type" is a technical term that groups atoms according to properties. Parameters are defined in terms of atom types, not in terms of elements or individual atoms (i.e. 
"atom names"). Thus the first step in parameter development is to decide which set of atoms will be assigned to the same atom type. A given element may, in different contexts, have quite different properties. Therefore, for example, an oxygen in a keto group is assigned to one atom type, while an oxygen in a hydroxyl group is assigned to another. In principle, almost every environment is unique, and so one could define a very large number of atom types. This would make force fields extraordinarily complicated and costly. In practice, a middle ground is sought in which atoms in quite different environments are assigned different atom types, while those in similar environments are assigned the same.

In the case of $\mathrm{CPD}$, it is reasonable to identify two carbon atom types, one oxygen atom type and one hydrogen atom type. I look for similar atom types in the CHARMM general force field parameter file. The carbon, oxygen and hydrogen atom types are similar to their respective atoms type in 2-pyrrolidinone - C1 and C4 of type CG2R53; C2, C3 and C5 of type CG3C52; O1 and O4 of type OG2D1; H21, H22, H31, H32, H51, H52 of type HGA2. The VDW parameters are not expected to change significantly and so I use the same values without refinement, as recommended by Mackerel.[72] The reason I look for similar atom types already present in the default parameter files is to avoid creating new atom types in the original topology and parameter files if possible. I could just as easily create completely new atom types since I modify all the parameters (except the VDW parameters since it is not expected it to change significantly) for CPD. It is worth noting that some thought must be given while deciding whether it is best to create new atom types or using existing atom types. If one uses existing atom types, like I did, then the new parameters will overwrite old parameters, if they already exist, in the 
parameter file. So, care must be taken that one does not intend using the old parameters for other molecules. In this case, some parameters are in fact overwritten, but in the context of my work I do not require the old parameters so it is safe for us to use the old atom types.

\begin{tabular}{|c|c|l|}
\hline Atom Name & Atom Type & \multicolumn{1}{c|}{ Description } \\
\hline C1 & CG2R53 & $\begin{array}{l}\text { 5-membered ring carbon bound to a keto } \\
\text { oxygen, e.g. in 2-pyrrolidinone }\end{array}$ \\
\hline O1 & OG2D1 & carbonyl oxygen; e.g. carboxylic acids \\
\hline C2 & CG3C52 & $\begin{array}{l}\text { 5-membered ring carbon bound to } \\
\text { aliphatic hydrogen e.g. in proline }\end{array}$ \\
\hline H21 & HGA2 & aliphatic hydrogen \\
\hline H22 & HGA2 & aliphatic hydrogen \\
\hline C3 & CG3C52 & $\begin{array}{l}\text { 5-membered ring carbon bound to } \\
\text { aliphatic hydrogen e.g. in proline }\end{array}$ \\
\hline H31 & HGA2 & aliphatic hydrogen \\
\hline H32 & HGA2 & aliphatic hydrogen \\
\hline C4 & CG2R53 & $\begin{array}{l}\text { 5-membered ring carbon bound to a keto } \\
\text { oxygen, e.g. in 2-pyrrolidinone }\end{array}$ \\
\hline OG2D1 & carbonyl oxygen; e.g. in carboxylic acids \\
\hline
\end{tabular}




\begin{tabular}{|c|l|l|}
\hline C5 & CG3C52 & $\begin{array}{l}\text { 5-membered ring carbon bound to } \\
\text { aliphatic hydrogen e.g. in proline }\end{array}$ \\
\hline H51 & HGA2 & aliphatic hydrogen \\
\hline H52 & HGA2 & aliphatic hydrogen \\
\hline
\end{tabular}

Table 1: CPD Atom Names and Atom Types

\subsubsection{Partial atomic charges}

The next step in the protocol is to assign initial guess partial charges to the different atoms. Each atom may in principle be assigned a different partial charge (i.e. the atom type does not determine the partial charge), but in general atoms in similar environments are assigned the same charge. Following the protocol of MacKerell et al. I start by assigning a +0.09 charge to all hydrogen atoms. The next step is to assign charges to the other atoms so that the total charge is zero, since the system is neutral. I determine an initial guess by calculating the charges using a quantum mechanical method. MacKerell et al. recommend using the HF/6-31G* level of theory to optimize the geometries and electronic wave functions of all molecules. This provides internal consistency for CHARMM parameter development. They recommend using the MerzKollman method [73]for determining the partial atomic charges. The Merz-Kollman method derives atomic charges from a least-squares fit to the electrostatic potential (ESP) calculated at a large number of points around the molecule of interest. The Merz-Kollman method differs from similar methods in the choice of points where the ESP is calculated. 
The MK scheme uses grid points on several layers around the molecule with different scaling factors for each layer. Keeping in mind the symmetry of the molecule and the fact that aliphatic hydrogen atoms need to have a +0.09 charge, I assign initial guess charges for all the atoms.

The next step is calculating target data for use in optimizing the partial atomic charges. These are obtained from quantum mechanical calculations. Two types of target data are calculated. One target datum is the dipole moment. The others pertain to the interaction between water molecules and CPD. In particular, the interaction energy and the optimal interaction distance are evaluated. An evaluation is made for each of the solvent-exposed atoms of CPD. This is accomplished by placing a single water molecule in close proximity to the atom under consideration, and performing the calculations described below. The partial atomic charges are then adjusted iteratively until the MM calculations well reproduce the dipole moment and water interactions obtained from QM calculations.
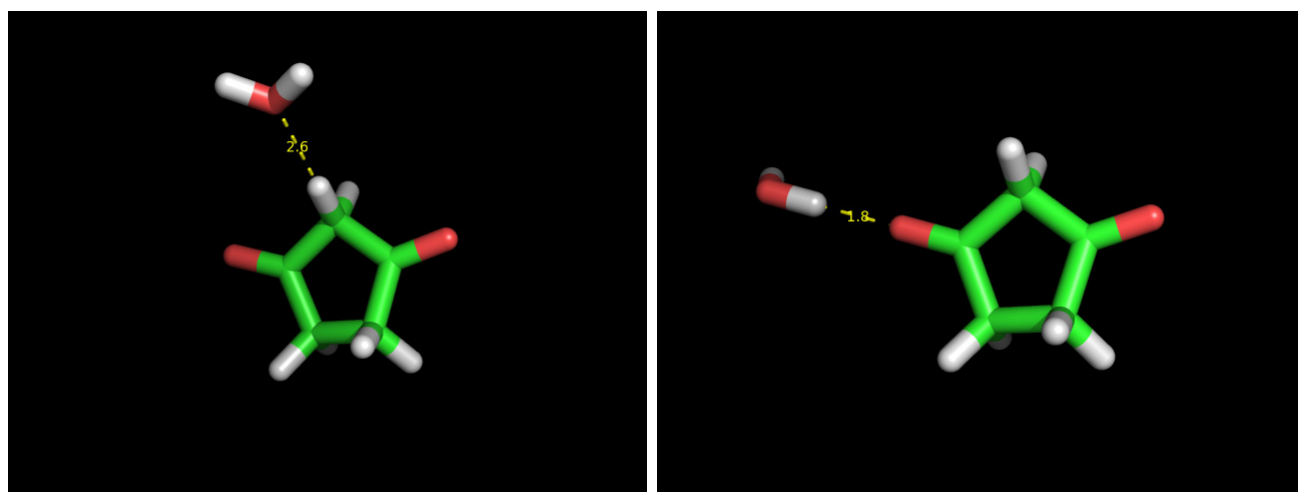

Figure 24: Two example representations of water interacting with atoms in CPD I begin with two structures; one optimized using QM and the other optimized using the CHARMM program for 200 minimization steps using the Conjugant Gradient 
algorithm and another 50 minimization steps using the Newton-Raphson algorithm. The QM optimized structure is copied in the comparison coordinate set and then the CHARMM optimized structure is aligned or oriented so that the dipole moments can be calculated and compared.

To get the other target data for water interaction energies and optimal hydrogen bond distances I first read in the QM optimized structure and build a TIP3P water molecule $1.6 \AA$ from one of the atoms. The TIP3P water molecule is a classical water model that I use in the solvation study. The energy is set at an arbitrary maximum value of $1000 \mathrm{kcal} / \mathrm{mol}$. The distance between the water molecule and the atom is gradually decreased by $0.01 \AA$ and the corresponding energy calculated. This is done until the minimum interaction energy is located. I follow the procedure for every periphery atom, with a single water molecule, one at a time. This data is then compared to the target data which I obtain from the QM calculation.

As mentioned above, the CHARMM General Force Field is an additive force field. One of the inherent disadvantages to an additive force field is the way electrostatic interactions are calculated. Coulomb's law is used to calculate the electrostatic interactions and uses fixed point charges on atoms to do so. Using fixed point charges is a major simplification. It is well known that the electron density or distribution around an atom and in this case atomic charges, changes as a function of the electrostatic field around the molecule. So, as the atoms move during simulation, their atomic charge should change or respond to its environment. This is ignored in an additive force field. To compensate for the lack of polarizability the atomic charges are 'tweaked' or 'enhanced', especially for molecules that are polar and in condensed phase. CPD is a polar molecule; 
hence, the target data acquired from QM calculations are 'enhanced'. One of the major developers of the CHARMM force fields, MacKerell et al., has outlined the modifications that need to be made to the target data. I follow these recommendations and make the following changes. The target interaction energies for the QM optimized structure are scaled by a factor of 1.16. The target optimal hydrogen bond distances are increased by about $0.2 \AA$. The target dipole moment is scaled by $0.8-0.9$.

The only parameters I can change at this step are the partial atomic charges on CPD. CPD is a symmetric molecule so we expect atoms like $\mathrm{C} 1$ and $\mathrm{C} 4$ to have the same charge. Likewise, $\mathrm{C} 2$ and $\mathrm{C} 3$ would have the same charge and also O1 and O4. The aliphatic hydrogen atoms need to have an atomic charge of +0.9 , as described earlier. Therefore, there are only four atomic charges I am able to manipulate, including $\mathrm{C} 1$ (or C4), $\mathrm{C} 2$ (or C3), $\mathrm{O} 1$ (or O4) and C5. Minor changes were made to these parameters and then the empirical data was compared to the target data. Several such iterations were done to approach the target data. The approach used to achieve this goal was to have the least possible deviation from the different types of target data like the dipole moment, water interaction energies and their respective minimum energy distance for all the atoms in CPD.

Ideally, the recommended level of agreement, for water interaction energies should be within $0.2 \mathrm{kcal} / \mathrm{mol}$ of the target values. The dipole moment should be overestimated by 20 to $50 \%$ in comparison to the QM calculated dipole moment. The best partial charges I was able to obtain are listed in Table 2 . 


\begin{tabular}{|c|c|c|c|}
\hline Atom Name & MK & Initial CHARMM & Final CHARMM \\
\hline C1 & 0.56 & 0.55 & 0.48 \\
\hline C2 & -0.18 & -0.18 & -0.19 \\
\hline C3 & -0.16 & -0.18 & -0.19 \\
\hline C4 & 0.55 & 0.55 & 0.48 \\
\hline C5 & -0.45 & -0.36 & -0.2 \\
\hline O1 & -0.46 & -0.46 & -0.46 \\
\hline O4 & -0.46 & -0.46 & -0.46 \\
\hline H21 & 0.07 & 0.09 & 0.09 \\
\hline H22 & 0.08 & 0.09 & 0.09 \\
\hline H31 & 0.07 & 0.09 & 0.09 \\
\hline H52 & 0.07 & 0.09 & 0.09 \\
\hline & 0.15 & & \\
\hline & 0.15 & & 0.09 \\
\hline
\end{tabular}

Table 2: CPD Partial atomic charge 
The interaction energies with water molecules, the respective hydrogen bond distances with the water molecule and the dipole moments are listed in Table 3. Note: the QM energies are scaled by 1.16 .

\begin{tabular}{|c|c|c|c|}
\hline & $\begin{array}{c}\text { QM } \\
\text { (kcal/mol) }\end{array}$ & $\begin{array}{l}\text { CHARMM } \\
\text { (kcal/mol) }\end{array}$ & $\underset{(\mathrm{kcal} / \mathrm{mol})}{\Delta(\text { CHARMM }- \text { QM })}$ \\
\hline $\mathrm{O} 1-\mathrm{HOH}$ & -4.1479 & -4.62955 & -0.48165 \\
\hline $\mathrm{O} 4-\mathrm{HOH}$ & -4.1479 & -4.6454 & -0.4975 \\
\hline $\mathrm{H} 21-\mathrm{OHH}$ & -3.0084 & -2.15067 & 0.85773 \\
\hline $\mathrm{H} 22-\mathrm{OHH}$ & -2.5196 & -1.68288 & 0.83672 \\
\hline $\mathrm{H} 31-\mathrm{OHH}$ & -2.5196 & -1.68288 & 0.83672 \\
\hline $\mathrm{H} 32-\mathrm{OHH}$ & -3.0084 & -2.15067 & 0.85773 \\
\hline $\mathrm{H} 51-\mathrm{OHH}$ & -3.29 & -1.84931 & 1.44069 \\
\hline \multirow[t]{3}{*}{$\mathrm{H} 52-\mathrm{OHH}$} & \multirow[t]{3}{*}{-3.29} & -1.84931 & 1.44069 \\
\hline & & AVG Difference & 0.661391 \\
\hline & & RMS Difference & 0.707352 \\
\hline
\end{tabular}

Table 3: $\mathrm{CPD}-\mathrm{H}_{2} \mathrm{O}$ Interaction Energies 


\begin{tabular}{|c|c|c|c|}
\hline & QM (A) & CHARMM (A) & $\begin{array}{c}\Delta \text { (CHARMM - QM) } \\
(\mathbf{A})\end{array}$ \\
\hline $\mathrm{O} 1-\mathrm{HOH}$ & 2.11 & 1.83 & -0.28 \\
\hline $\mathrm{O} 4-\mathrm{HOH}$ & 2.11 & 1.83 & -0.28 \\
\hline $\mathrm{H} 21-\mathrm{OHH}$ & 2.47 & 2.64 & 0.17 \\
\hline $\mathrm{H} 22-\mathrm{OHH}$ & 2.47 & 2.66 & 0.19 \\
\hline $\mathrm{H} 31-\mathrm{OHH}$ & 2.47 & 2.66 & 0.19 \\
\hline $\mathrm{H} 32-\mathrm{OHH}$ & 2.47 & 2.64 & 0.17 \\
\hline $\mathrm{H} 51-\mathrm{OHH}$ & 2.34 & 2.63 & 0.29 \\
\hline $\mathrm{H} 52-\mathrm{OHH}$ & 2.34 & 2.63 & 0.29 \\
\hline
\end{tabular}

Table 4: $\mathrm{CPD}-\mathrm{H}_{2} \mathrm{O}$ Interaction Distances

\begin{tabular}{|c|c|}
\hline QM (D) & CHARMM (D) \\
\hline 1.8625 & 1.8152 \\
\hline
\end{tabular}

Table 5: Dipole moments 


\subsubsection{Bond and angle terms}

The next step is the optimization of intra-molecular bond and angle parameters. The functional forms for these terms are shown below.

$$
\sum_{\text {bonds }} K_{b}\left(b-b_{0}\right)^{2}+\sum_{\text {angles }} K_{\theta}\left(\theta-\theta_{0}\right)^{2}
$$

Bond and angle parameters are optimized at the same time. There are two values needed for bond and angle parameters, the equilibrium values and the force constants. The equilibrium values can easily be obtained from a corresponding QM optimization of the molecule. The MP2/6-31G* level of theory is recommended for this purpose. Ideally, deviations of up to $0.03 \AA$ for equilibrium bond distances and up to $3^{\circ}$ for equilibrium angles in comparison to QM calculations is acceptable. The optimization of the force constants, however, is quite involved and was possibly the longest and most tedious part of the optimization process for CPD. CHARMM uses a module called MOLVIB, which is a general-purpose vibrational analysis program used for small molecules that contain fewer than 50 atoms.

The MOLVIB module is very structured and the syntax has to be closely followed. The MOLVIB module is also used to get the target data, but the target force matrix for the vibrational analysis is obtained from a QM calculation of a frequency calculation. The force matrix from the QM (Gaussian98) output is then copied into the MOLVIB with the target frequencies scaled by a factor of 0.89 . 
The force constants are adjusted so that they best match the target data. The goal is to reproduce the frequencies and the contributions of the internal degrees of freedom. Lower frequencies represent motions involving heavy atoms and, therefore, motion that deviates the most from its optimal geometry. Hence, it is a priority to reproduce the low frequencies more accurately. The high frequency motion that represent stretching motion involving hydrogen atom are trivial and in most cases not important. It is preferable to produce vibrational frequencies that are slightly lower than the target values. The reasoning is that it is better to have a more flexible molecule instead of a more rigid molecule.

As a rough guideline the empirical frequencies calculated should be, on average, within 5\% of the corresponding QM calculated frequencies. "Apart from these basic guidelines, it is difficult to objectively quantify the quality of the parameterization." "It is often difficult to unambiguously correlate the $\mathrm{QM}$ and $\mathrm{MM}$ frequencies due to multiple local internal valence coordinate contributions to each frequency. These contributions will often differ between the QM and MM spectra." [72]. 


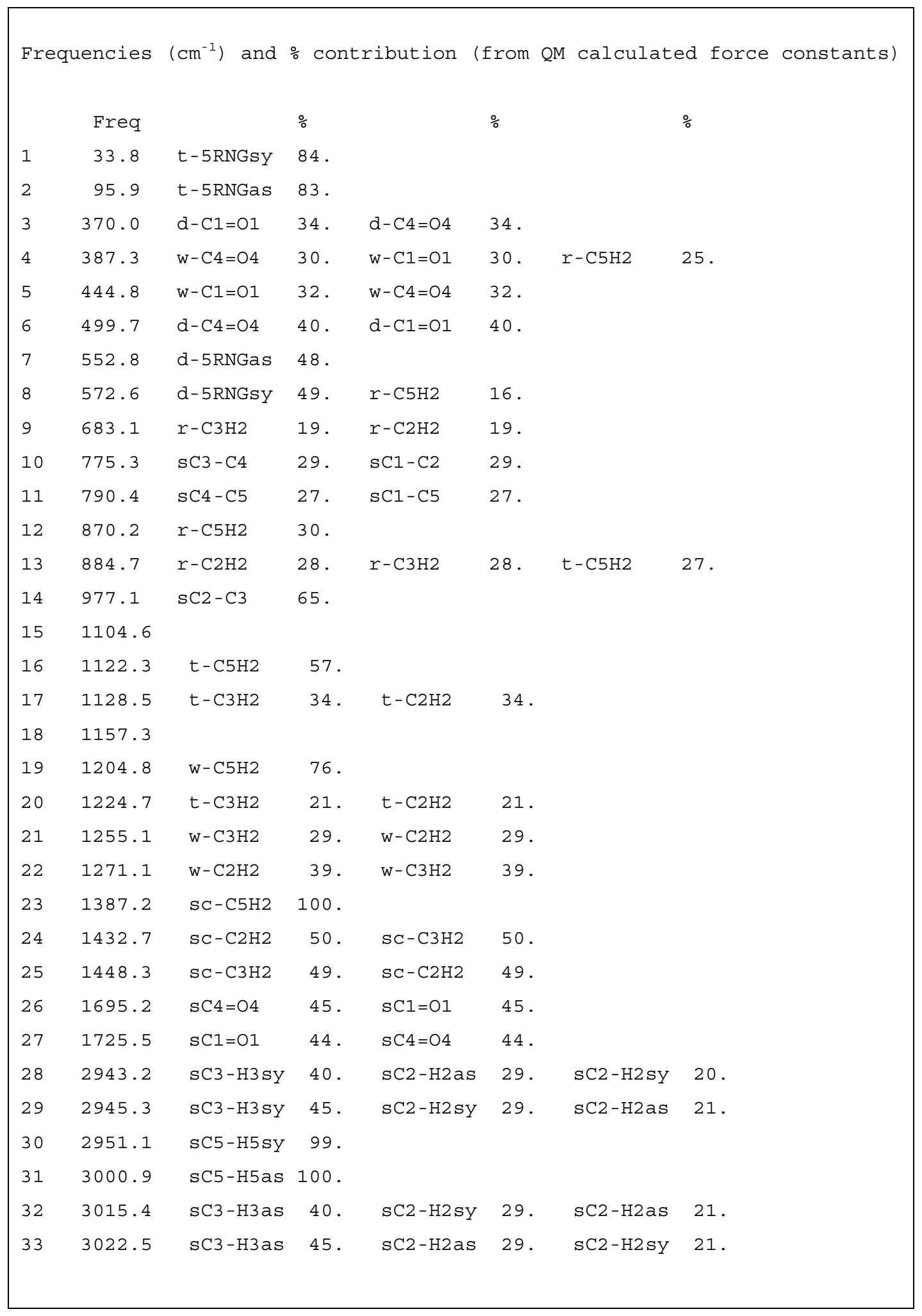

Table 6: QM calculated Frequencies $\left(\mathrm{cm}^{-1}\right)$ and $\%$ contribution 


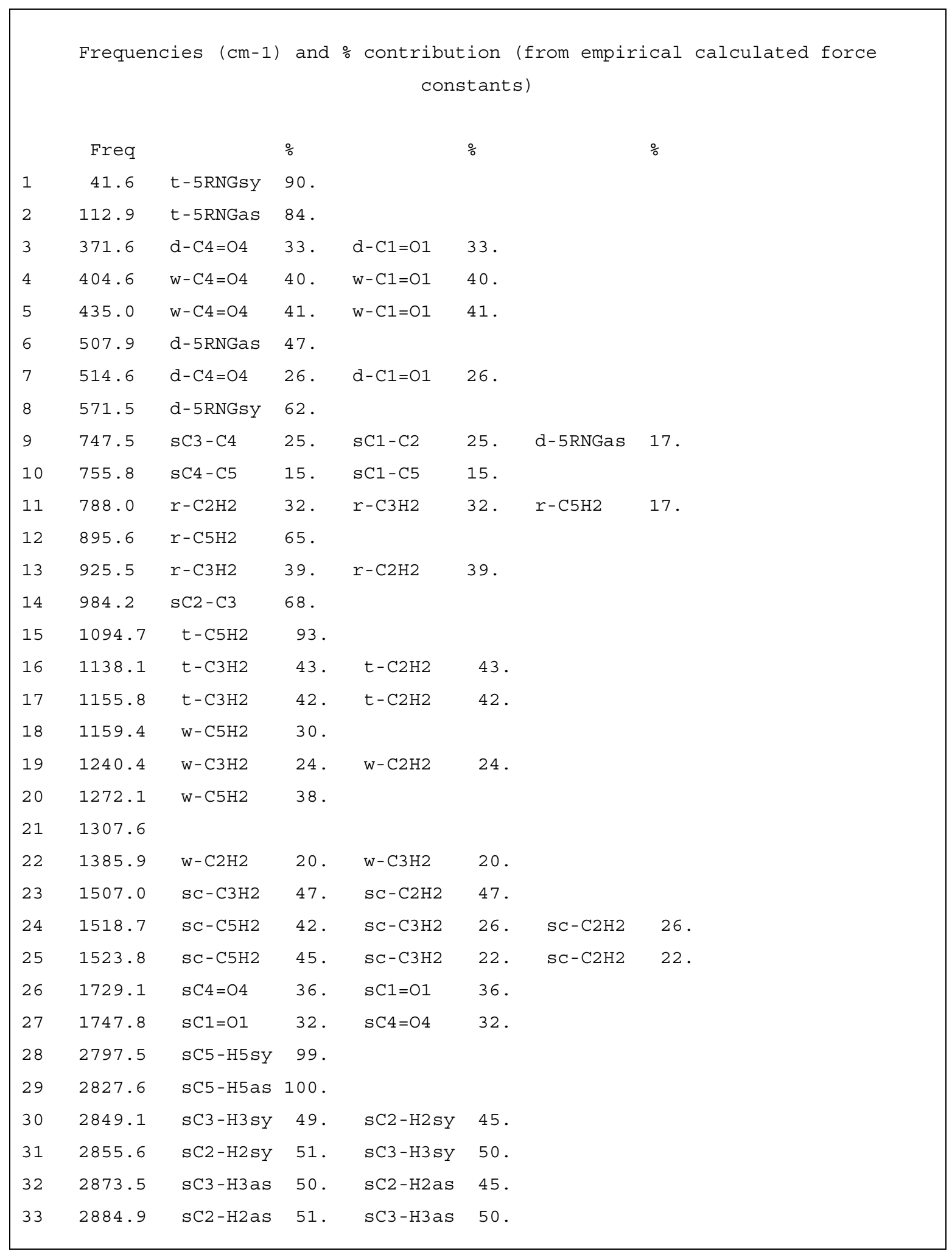

Table 7: MM calculated Frequencies $\left(\mathrm{cm}^{-1}\right)$ and \% contribution 


\subsubsection{Dihedral and improper terms}

The last two sections of the parameter optimization are the dihedral and improper angles and force constants. The torsions are one of the degrees of freedom that are optimized while optimizing the bond and angle force constants. The dihedrals that have no hydrogen are fine-tuned next. The optimization of dihedrals is designed to best reproduce the QM potential energy surface (PES). The QM calculations use the MP2 level of theory and the $6-31 \mathrm{G}^{*}$ basis set for the target data.

All the dihedrals are held fixed, while the one under consideration is restrained to a certain value and the energy calculated after a short minimization. The dihedral under consideration is then restrained to a new value, while still keeping the other dihedrals fixed, and the energy is again calculated after a short minimization. This is done for a set of several restrained values for the dihedral under consideration till a PES is mapped out. The short minimization carried out consists of 50 conjugate gradient algorithm steps and 100 Newton-Raphson algorithm steps. For example, the improper angle is first restrained to -45.0 degrees, the system minimized and energy calculated. The improper is angle is then restrained to -44.0 degrees, system minimized and energy calculated. This is done until a PES is obtained for the improper angle 'scan' in 1.0 degree increments from -45.0 degrees to +45.0 degrees. Similarly, I get the PES for all the dihedrals I intend to optimize, one at a time. The regular dihedral angles were restrained from -90.0 degrees to +90.0 degrees in 3.0 degree increments. Emphasis is placed on reproducing low energy regions and low barriers, since high barriers will not be crossed during typical room temperature MD simulations. I get a similar PES from QM calculations for the target 
data. Just like the other parameter optimization sections I try to reproduce the target data by adjusting the dihedral parameters.

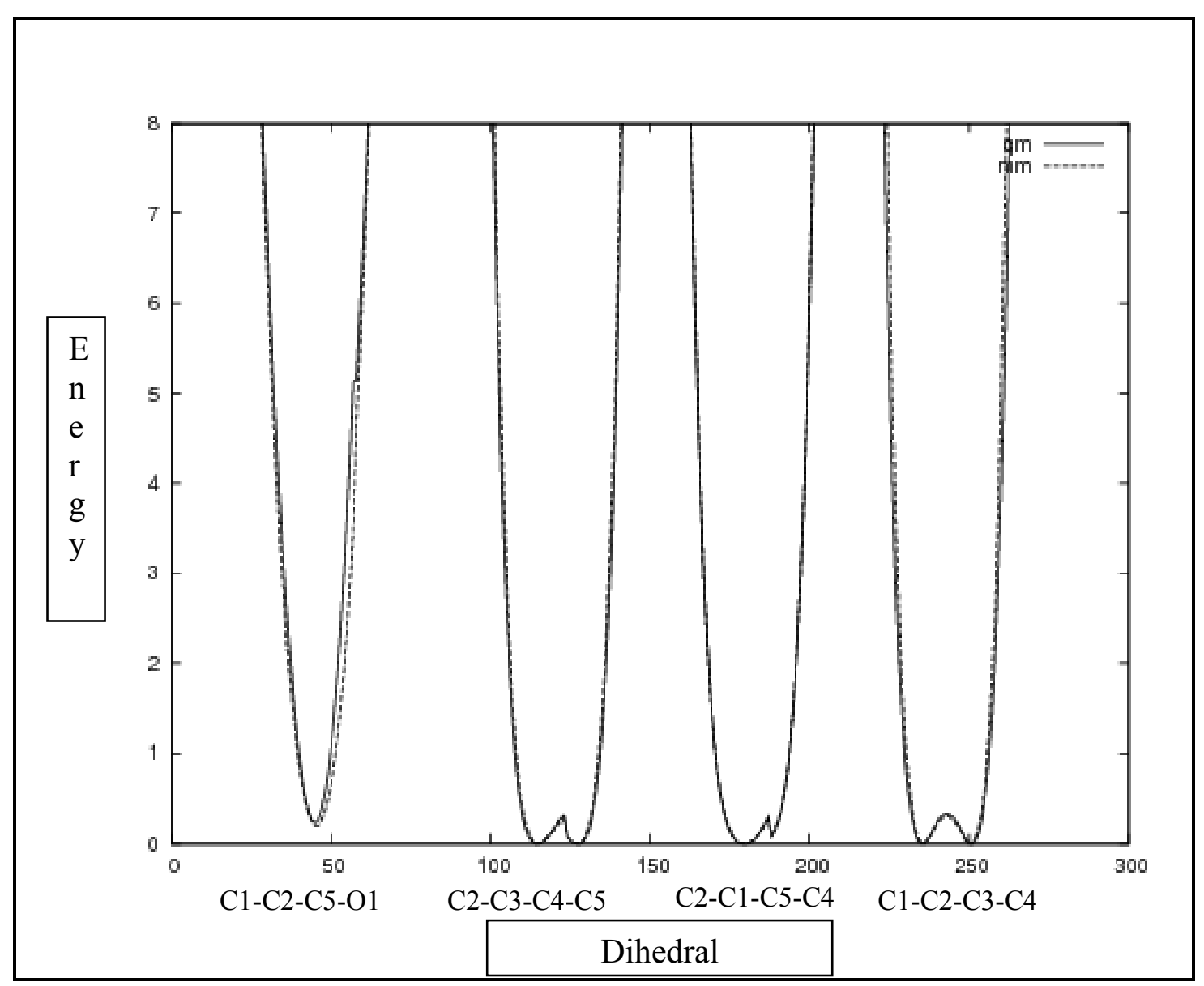

Figure 25: Dihedral potential energy 


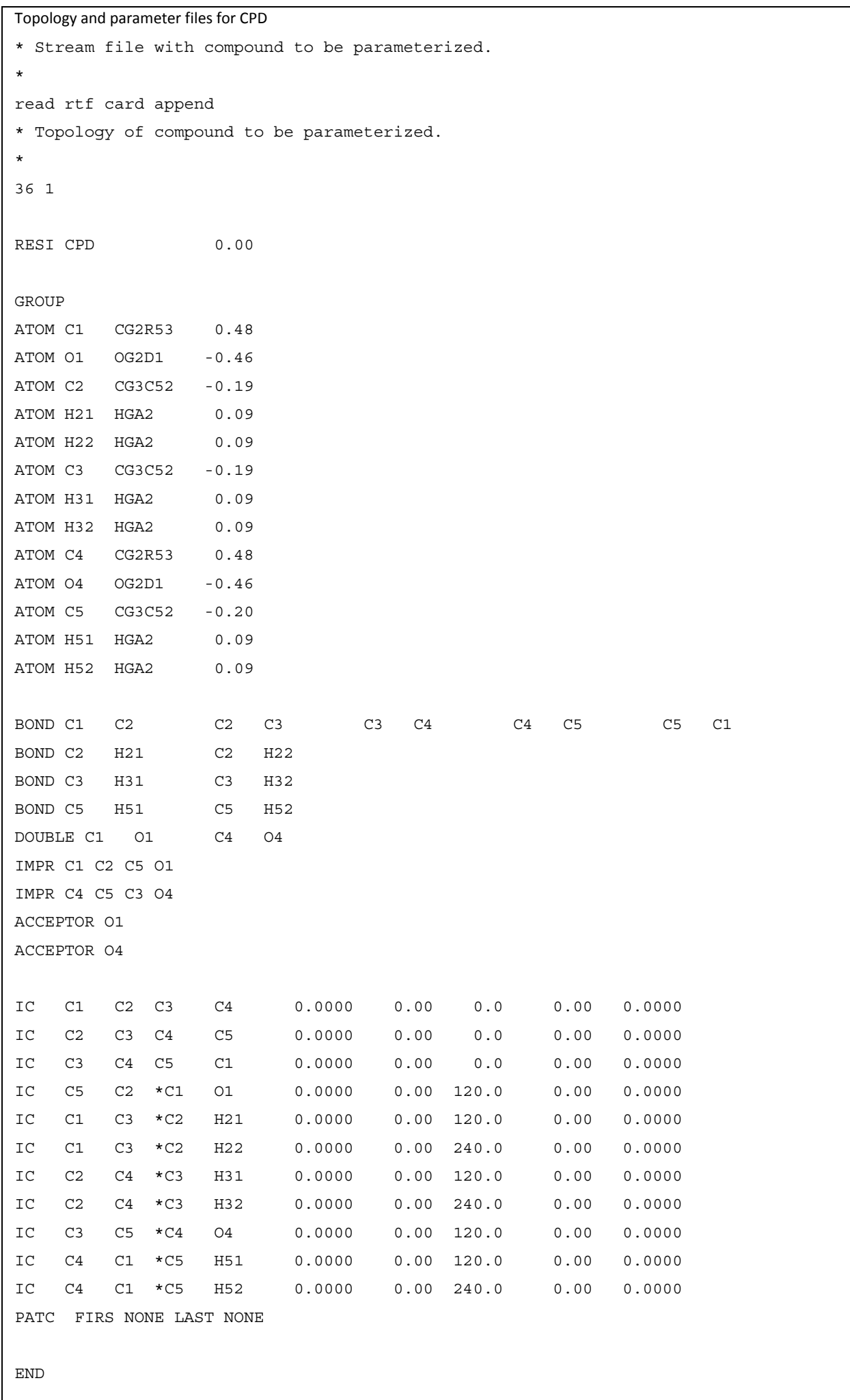

Table 8: CHARMM Topology File for CPD 


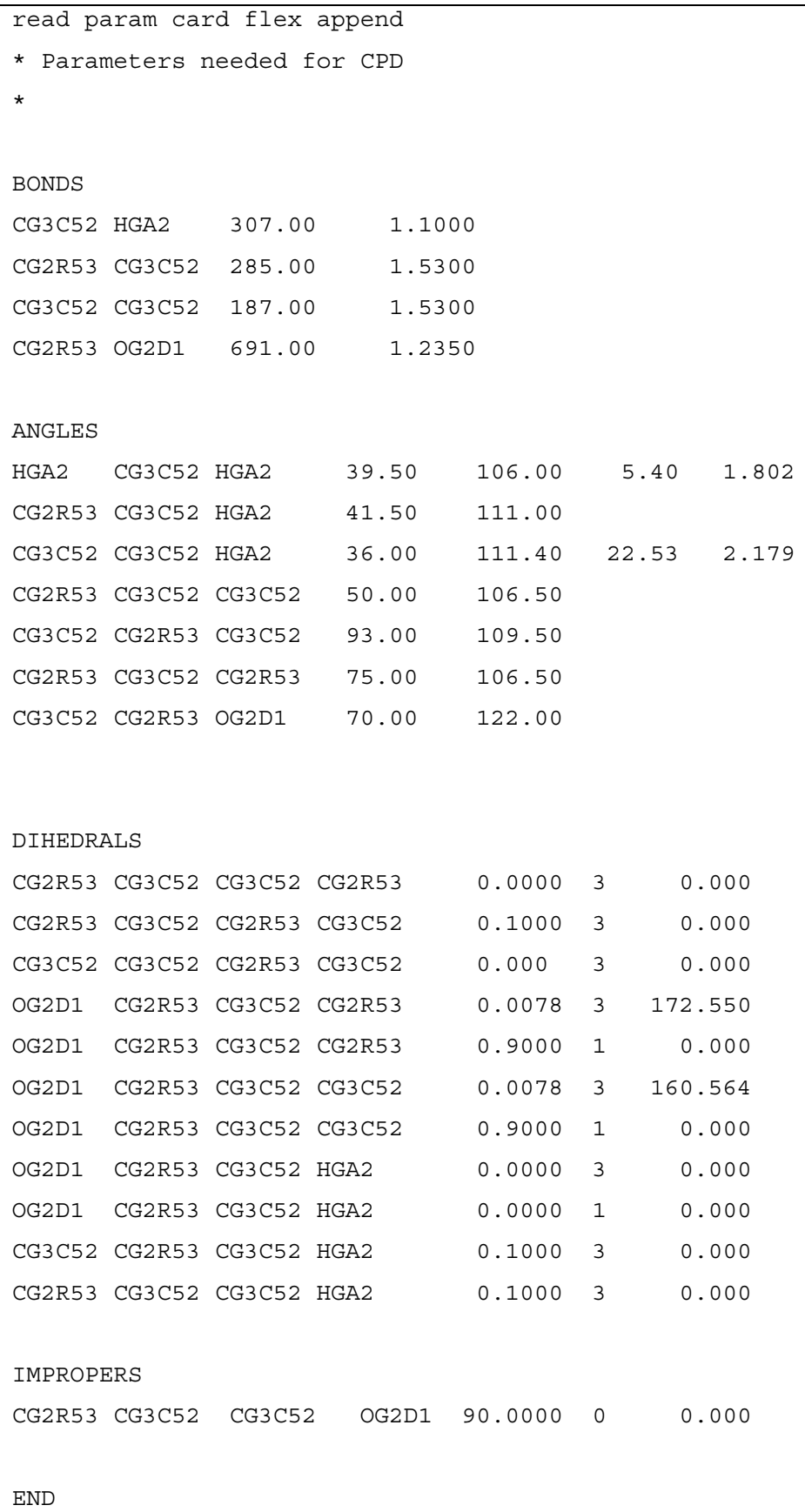

Table 9: CHARMM Parameter File for CPD 


\section{2. $\mathrm{HOCl}$}

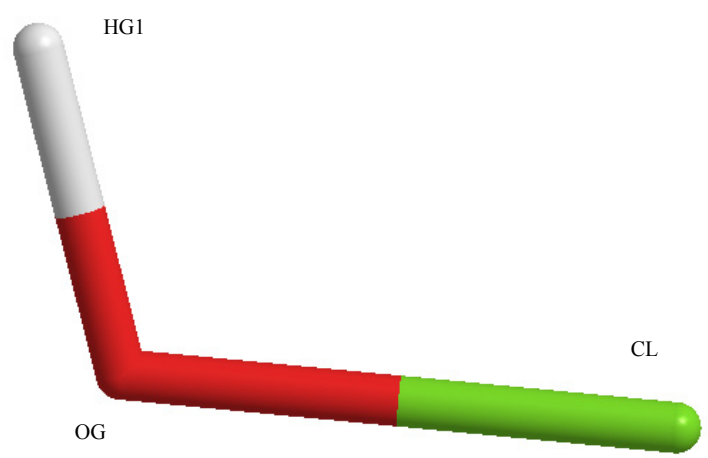

Figure 26: Hypochlorous acid (HOCl). Atom names are shown

Parameters for $\mathrm{HOCl}$ were also developed from the ground up, using the procedure described in detail for $\mathrm{CPD}$. Developing parameters for $\mathrm{HOCl}$ was simpler than for $\mathrm{CPD}$ since $\mathrm{HOCl}$ has only three atoms and three atom types. Obviously, no dihedral optimization is needed and only two bond parameters and one angle parameter in addition to the partial atomic charges are needed. The van der Waals parameters are taken directly from similar atom types in the existing parameter file and not modified, as for CPD and as recommended. The finalized parameters are given in Appendix. Table 11 and Table 12 below compares QM and MM observables.

\begin{tabular}{|c|c|c|}
\hline Atom Name & Atom Type & \multicolumn{1}{|c|}{ Description } \\
\hline $\mathrm{CL}$ & CLGA1 & $\begin{array}{l}\text { Chlorine e.g. Mono and dichloro } \\
\text { ethane }\end{array}$ \\
\hline
\end{tabular}




\begin{tabular}{|c|c|l|}
\hline OG & OG311 & hydroxyl oxygen \\
\hline HG1 & HGP1 & polar hydrogen \\
\hline
\end{tabular}

Table 10: HOCl Atom Names and Atom Types

\begin{tabular}{|c|c|c|c|}
\hline & $\begin{array}{c}\mathbf{Q M} \\
\mathbf{( k c a l / m o l})\end{array}$ & $\begin{array}{c}\text { CHARMM } \\
\text { (kcal/mol) }\end{array}$ & $\begin{array}{c}\Delta \text { (CHARMM - QM) } \\
\text { (kcal/mol) }\end{array}$ \\
\hline $\mathrm{H}-\mathrm{OHH}$ & -9.6396 & -6.61081 & 3.02879 \\
\hline $\mathrm{O}-\mathrm{HOH}$ & -2.76436 & -3.6361 & -0.87174 \\
\hline \multirow{2}{*}{$\mathrm{Cl}-\mathrm{OHH}$} & -2.6294 & -0.588162 & 2.041238 \\
\hline & & AVG Difference & 1.39943 \\
\cline { 3 - 5 } & & RMS Difference & 1.65579 \\
\cline { 3 - 4 } & & &
\end{tabular}

Table 11: $\mathrm{HOCl}-\mathrm{H}_{2} \mathrm{O}$ Interaction Energies

\begin{tabular}{|c|c|c|c|}
\hline & $\begin{array}{c}\text { QM } \\
\text { (kcal/mol) }\end{array}$ & $\begin{array}{c}\text { CHARMM } \\
\text { (kcal/mol) }\end{array}$ & $\begin{array}{c}\Delta \text { (CHARMM - QM) } \\
\text { (kcal/mol) }\end{array}$ \\
\hline $\mathrm{H}-\mathrm{OHH}$ & 1.86 & 1.86 & 0 \\
\hline $\mathrm{O}-\mathrm{HOH}$ & 1.97 & 1.97 & 0 \\
\hline $\mathrm{Cl}-\mathrm{OHH}$ & 2.92 & 3.53 & 0.61 \\
\hline
\end{tabular}

Table 12: $\mathrm{HOCl}-\mathrm{H}_{2} \mathrm{O}$ Interaction Distances 


\begin{tabular}{|c|c|}
\hline QM (D) & CHARMM (D) \\
\hline 1.8253 & 1.96711 \\
\hline
\end{tabular}

Table 13: Dipole moments

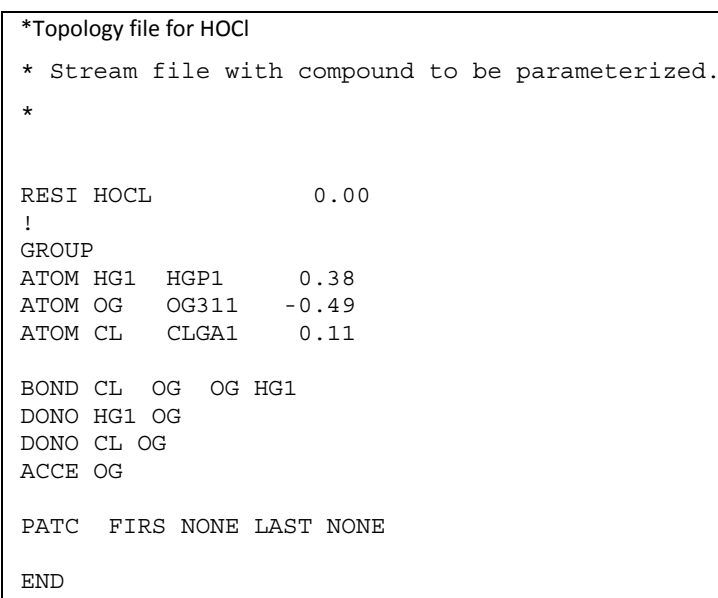

Table 14: CHARMM Topology File for HOCI

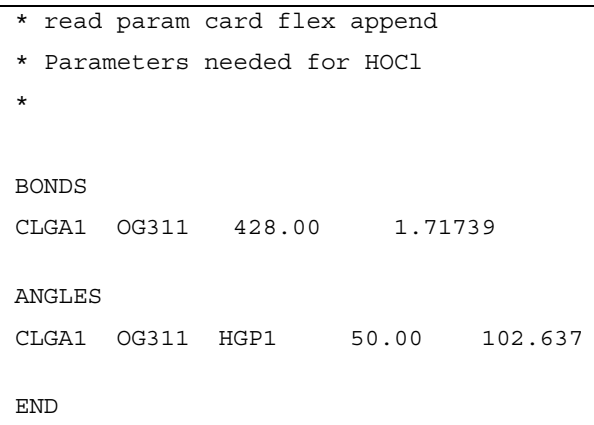

Table 15: CHARMM Parameter File for HOCI 


\subsection{CPO sugars}

Sugar parameters were developed using the analogy method, for two reasons. First, the CHARMM force field contains parameters for some related sugars, and these parameters are expected to be largely transferable to the other sugars present in Chloroperoxidase. Second, the sugars themselves are located at the periphery of CPO, far from the active site where reaction takes place. Thus calculations of the reaction barrier for epoxidation at the active site or chlorination, if it occurs at the active site, are not expected to be very sensitive to the sugar parameters, and approximate parameters would be sufficient under such circumstances.

Application of the analogy method involves minor modification of atoms or atom types that would not significantly affect the energetic. This requires some chemical intuition. The process by which sugar parameters for addition to the default CHARMM topology file is described below.

\subsubsection{Alpha-D-xylopyranose (AXYS)}

Alpha-D-xylopyranose is similar to alpha-D-glucose (AGLC), which is already present in the CHARMM sugar topology file. All that is required to construct AXYS is to remove a small group of atoms from AGLC and replace them with hydrogen atoms. There are a few important details, so I describe the procedure carefully below.

I make a new entry for AXYS in the topology file. The AGLC section in the topology file is copied for AXYS, keeping only the atoms that are common to both molecules. Then the two extra hydrogen atoms are added to complete the AXYS 
molecule. The following atoms from AGLC are deleted: C6, H61, H62, O6, HO6. The atoms H51 and H52 are added. Partial atomic charges must be adjusted slightly so that the individual charges remain consistent and the overall charge is zero. In the process of adjusting charges, the aliphatic hydrogen atoms are given charges of +0.09 as recommended by MacKerell. Carbon atom C5 has its charge adjusted to +0.250 to accommodate overall charge neutrality.

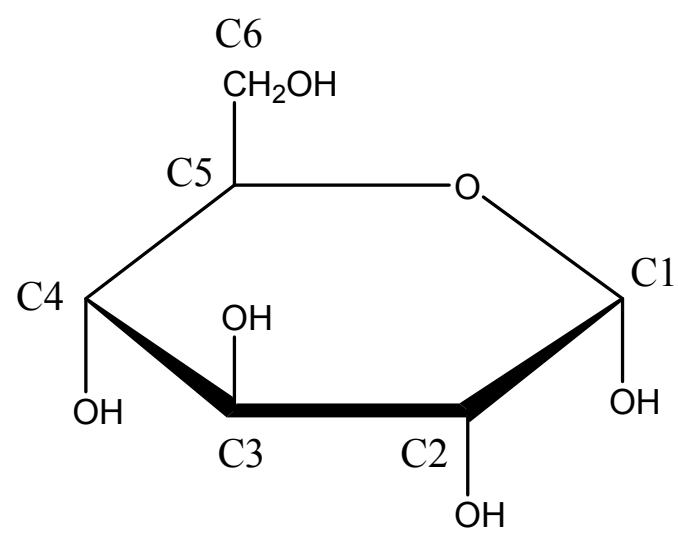

(a)

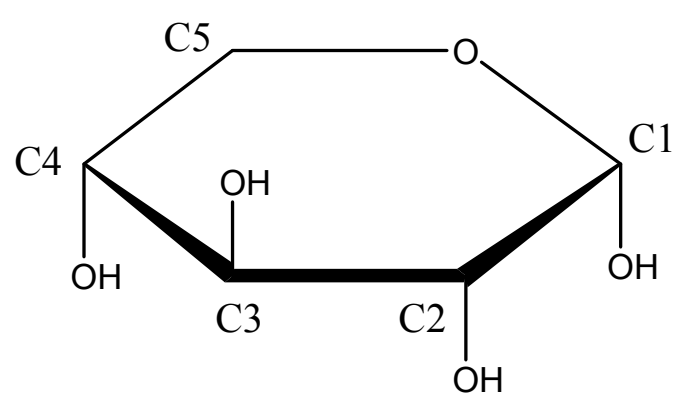

(b)

Figure 27: Sugar: (a) alpha-D-glucose (b) alpha-D-xylopyranose

Adjustments are made in the 'BOND' section of the topology file to provide information about which atoms are bonded. The donor ('DONO'), acceptor ('ACCE') and internal coordinate (IC) table sections are also modified. This is not absolutely necessary, though, because the information they provide can be included in a CHARMM input script if required. 


\subsubsection{Beta-L-xylopyranose (BXYS)}

The molecule BXYS and AXYS are similar. Therefore all parameters for BXYS are the same as for AXYS; only the coordinates or stereochemistry are different. An $\alpha$ or $\beta$ sugar are defined by the stereochemistry of the first carbon (C1) with respect to the last

chiral carbon atom (C4). If they have the opposite stereochemistry the sugar is define as $\alpha$ and if they both have the same stereochemistry the sugar is defined as $\beta$.

We do not need to consider the orientation or the cartesian coordinates in the topology file (they are gotten from the Protein Data Bank (PDB) or a coordinate (CRD) file). Consequently, all that is needed is to create an entry for BXYS and copy all the parameters directly from AXYS.

\subsubsection{Beta-L-arabinose(BARB)}

Like BXYS, BARB is similar to AXYS. Essentially, only the coordinates differ between BARB and AXYS; the parameters are again identical. A new entry BARB is created with the AXYS parameters copied in. 


\subsubsection{N-Acetyl-Glucosamine (BNAG)}

Like AXYS, BNAG is similar to a sugar already in the parameter set, alpha-Dglucose (AGLC). Thus parameterization of BNAG requires a small modification of AGLC parameters. However, in this case the hydroxyl group attached to $\mathrm{C} 2$ is replaced by $-\mathrm{NHCOCH} 3$ group. The new group is split in to three sub-groups $\mathrm{NH}, \mathrm{CO}$ and $\mathrm{CH} 3$, each with a net partial charge of zero.

\subsubsection{Pyroglutamic acid (PCA)}

Pyroglutamic acid is an uncommon amino acid and is a terminal amino acid in CPO. It is similar to a more common amino acid, glutamic acid. The terminal hydroxyl is missing in PCA and the delta-carbon in glutamic acid is connected to the backbone nitrogen to form a ring structure in PCA.

\subsection{Patches}

Patches are tools used in CHARMM when individual residues or molecules are required to be connected to others. These connections can be made explicitly in the CHARMM input script, but if this needs to be done regularly and often, it is worth creating a patch to do so. 
A patch usually contains two residues that are to be connected. Often some atoms need to be deleted; bond, angle and perhaps dihedral terms need to be added; and in some cases partial charges of the atoms need to be adjusted to maintain the overall charge deletion of atoms.

In the macromolecule $\mathrm{CPO}$ there are several sugar molecules that are connected to each other and also to the enzyme. To accomplish this in the model, several patches were created in the topology file. They are to be invoked in the input script when the entire enzyme is built. We also need a patch to connect the heme to the proximal ligand cysteine (CYS). An example of one patch is given below, and the others are given in the Appendix.

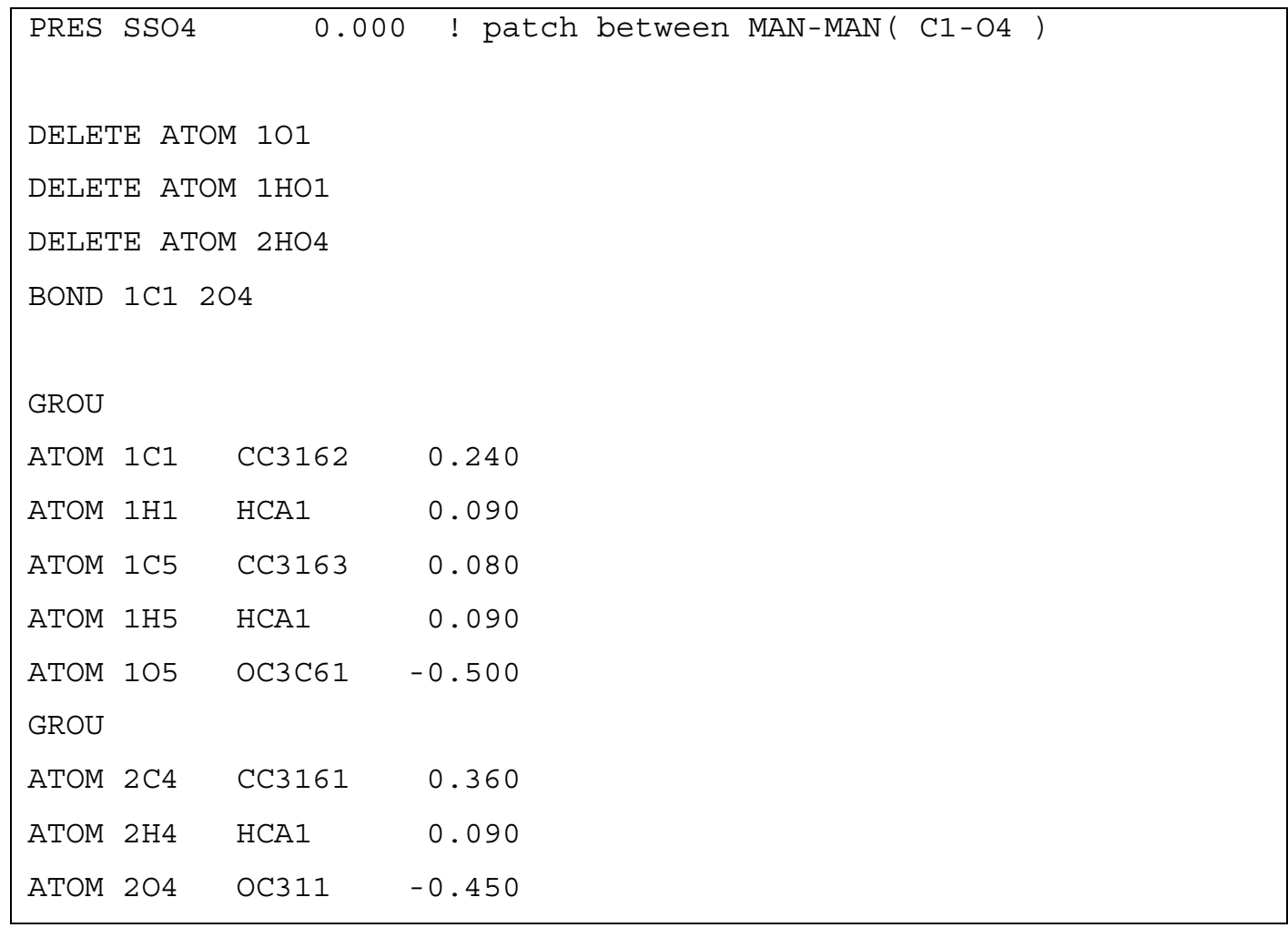

Table 16: Patch to connect two mannose sugar molecules 


\subsection{Conclusions}

In summary, I developed a set of molecular mechanics parameters for two molecules used in the study of the CPO halogenation mechanism and a set of sugars used in the study of the CPO epoxidation mechanism. My modeling work uses the CHARMM

parameter set and therefore, to be consistent, I followed the protocol outlined by MacKerell et al. to develop parameters. I developed parameters for CPD and $\mathrm{HOCl}$ from the ground up because precise parameters were needed. The parameters developed were tested and met the requirements outlined in the protocol by MacKerell et al [72].

I used the less time-consuming analogy method to develop parameters for sugars. The sugar structures are very similar to other sugars already in the default CHARMM parameter set, and these sugars are not close to the enzyme active site and do not require a high level of accuracy. For these reasons I expect the analogy method to be satisfactory in this case. Patches were developed to create heme-cysteine, sugar-sugar and sugaramino acid covalent bonds. 


\section{STUDY OF CPD DIKETO $\rightarrow$ KETO-ENOL TAUTOMERIZATION WITH LIMITED HYDRATION}

\subsection{Gas phase / Intramolecular proton transfer}

I begin for reference with the intra-molecular hydrogen transfer process, in which a hydrogen is transferred from the alpha-carbon to one of the keto oxygens of CPD. As expected the adiabatic reaction barrier is quite high, $63.9 \mathrm{kcal} / \mathrm{mol}$. Reactant, transition state and product structures are shown in Figure 28. Single-point calculations using the PCM method to model solvation implicitly do not alter the barrier heights significantly. The PCM barrier is $64.6 \mathrm{kcal} / \mathrm{mol}$.

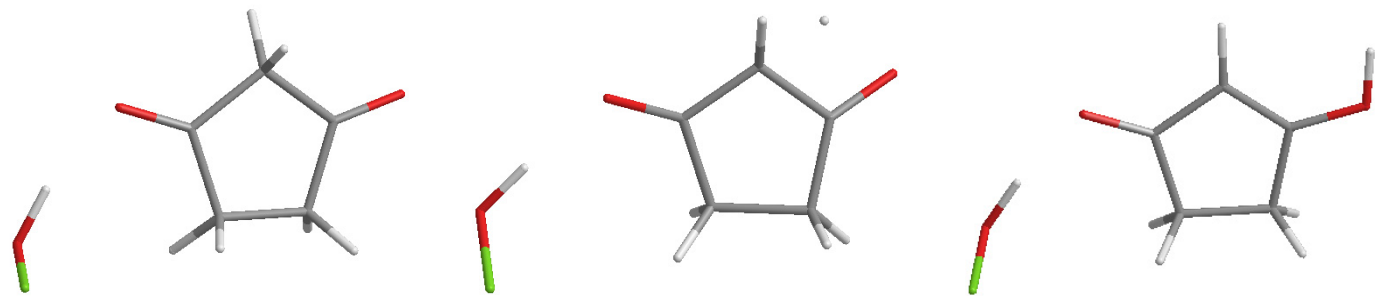

Figure 28: Intramolecular gas phase reaction. Stationary points. HOCI present to play stabilizing role representative of acidic conditions

\subsection{Intermolecular proton transfer with one catalytic water molecule}

When one catalytic water molecule is included, the reaction barrier decreases to $27.7 \mathrm{kcal} / \mathrm{mol}$. Reactant, transition state and product structures are shown in Figure 29. 
The water-catalyzed mechanism is a two-step process, with nearly complete abstraction of the alpha hydrogen by the water preceding transfer of a hydrogen from the water to the keto oxygen. As with intra-molecular hydrogen transfer, the implicit solvation model PCM method single-point calculation did not alter the barrier height significantly. The PCM barrier for the intermolecular hydrogen transfer is $26.8 \mathrm{kcal} / \mathrm{mol}$.

The ketoenol product is slightly more stable that the diketo reactant in both the uncatalyzed and the single-water-catalyzed reactions (Table 17); PCM-modeled solvation increases the degree of product stabilization.
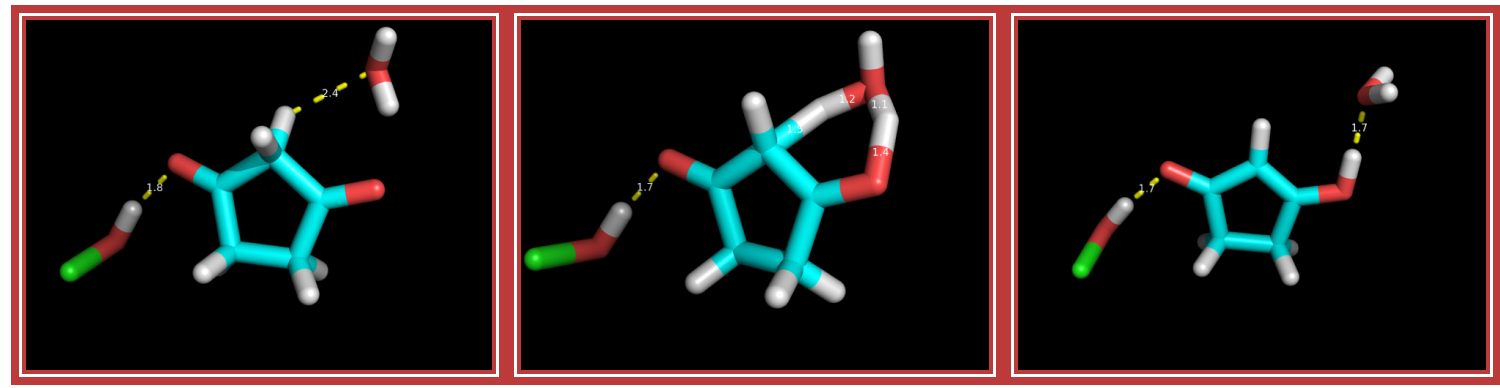

Figure 29: Intermolecular gas phase reaction. Stationary points. HOCl present to play stabilizing role representative of acidic conditions

\subsection{Water clusters, fully QM optimized}

Previous work on related systems has shown that additional explicit waters can lower the reaction barrier significantly [43-45]. Therefore I systematically evaluated the effect of additional hydration on the reaction barrier. To do so, I constructed clusters of water molecules surrounding the $\mathrm{CPD} / \mathrm{HOCl}$ system and determined the adiabatic reaction barriers, treating the entire system at the QM level. Systems with multiple 
waters possess a large number of degrees of freedom and great flexibility. Therefore the potential energy surface (PES) has a high dimensionality and many local minima. Meaningful, interpretable results require careful placement of waters. My approach was to begin with the stationary points of the single-water system and add water molecules incrementally. The water molecules were placed in highly favorable positions for forming hydrogen bonds. For reactants, the waters were added incrementally until the hydrogen bonding positions around the keto oxygens, the $\mathrm{HOCl}$ and the catalytic water were saturated, that is, until these hydrophilic atoms had one full solvation shell of explicit waters. I attempted to maintain similar degrees of hydration of these three moieties as I increased the level of hydration. To increment the degree of hydration, one or two new waters were added to a previously minimized reactant structure, and this structure was fully minimized. The same procedure was applied to the corresponding product and transition state structures. With this procedure, trends in barrier heights and reaction energies exhibited a systematic relation to level of hydration. When incremental waters were not carefully placed, however, trends were not systematic and were difficult to interpret (data not shown).

The results are shown in Table 17 and Figure 34. The overall reaction energy, $\Delta \mathrm{E}$, decreases from $-3.1 \mathrm{kcal} / \mathrm{mol}$ for a single catalytic water to $-17.2 \mathrm{kcal} / \mathrm{mol}$ for eight waters. Eight explicit waters are sufficient to model the overall reaction energetics, as $\Delta \mathrm{E}$ is nearly unchanged beyond eight waters. The reaction barrier exhibits a stronger dependence on level of hydration, and in a way that is critically dependent on the placement of incremental waters. The barrier height is 27.7 and $27.6 \mathrm{kcal} / \mathrm{mol}$ with one or three explicit waters, respectively. Waters two and three were placed so as to hydrate 
the $\mathrm{HOCl}$, having little effect on the reaction barrier. However, the barrier decreases rapidly as more explicit waters are added. Waters four and five were placed so as to hydrate the catalytic water, and thus had a substantial effect on the barrier. For systems with more than five water molecules, I was unable to fully optimize the transition states. This difficulty probably stems from the large number of highly flexible degrees of freedom and consequently quite flat PES in the vicinity of stationary points.
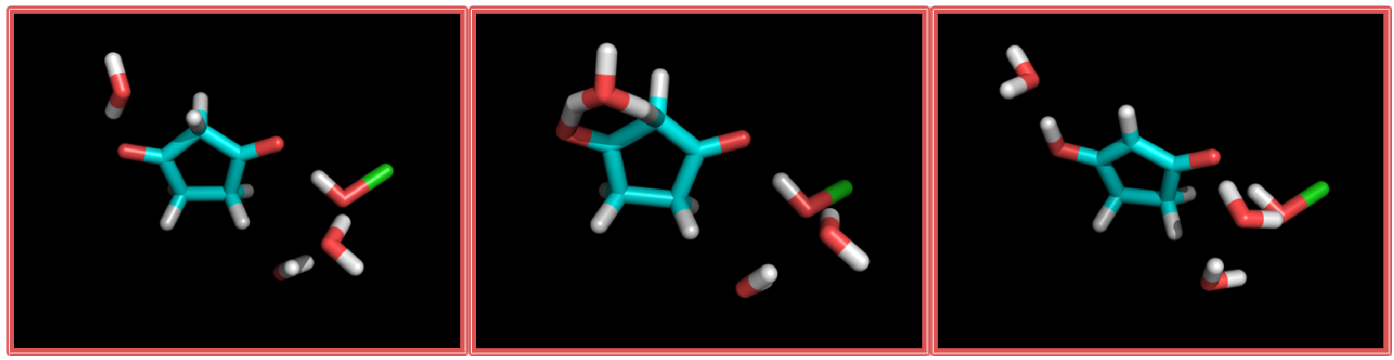

Figure 30: Stationary points of system with 3 explicit water molecules
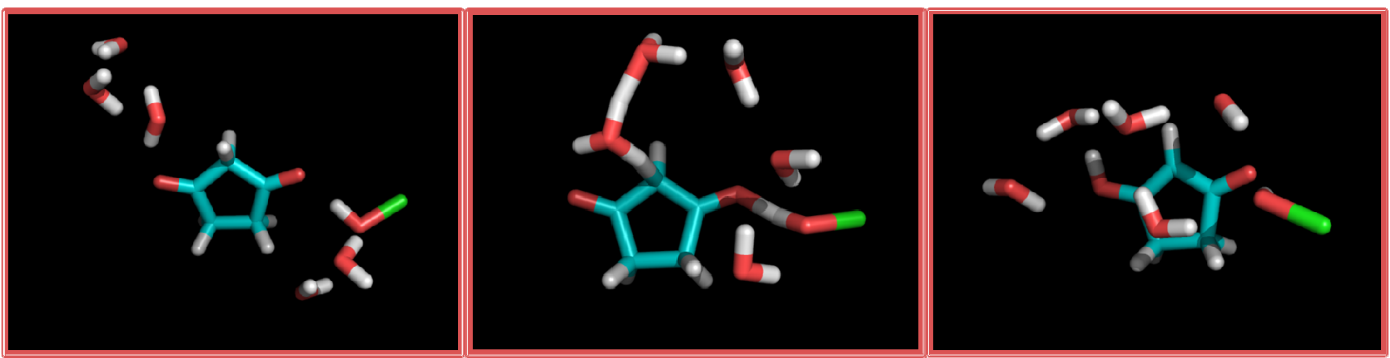

Figure 31: Stationary points of system with 5 explicit water molecules
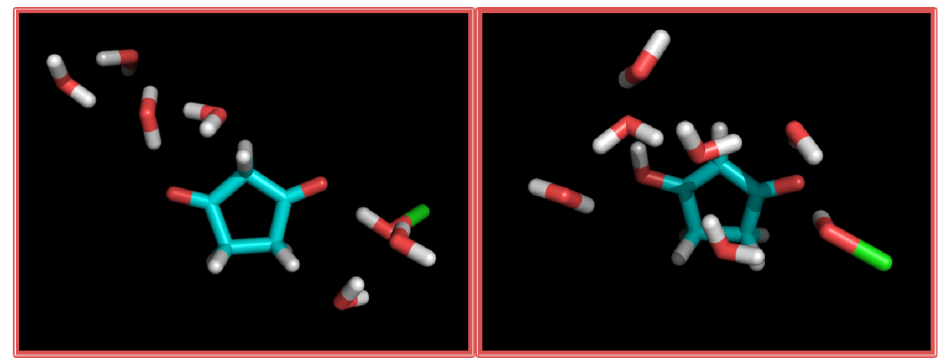

Figure 32: Stationary points of system with 6 explicit water molecules 


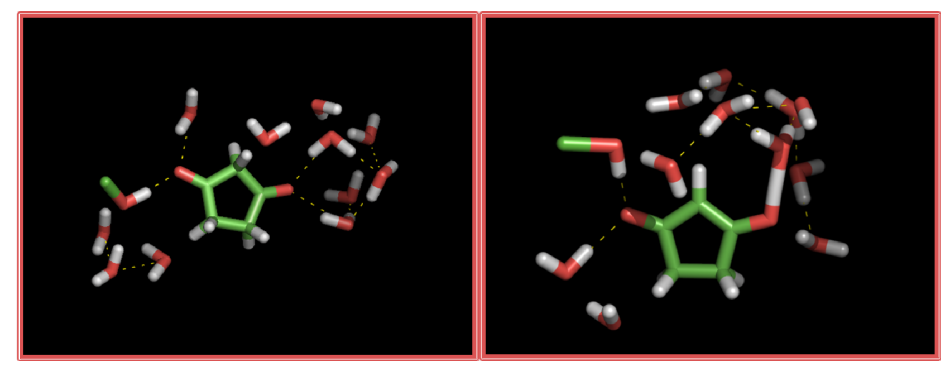

Figure 33: Stationary points of system with 11 explicit water molecules

For each stationary point at each hydration level, I also performed single-point energy calculations using the PCM method. The reaction energies changed by up to 8.3 $\mathrm{kcal} / \mathrm{mol}$. However, the barrier heights changed by no more than $1.6 \mathrm{kcal} / \mathrm{mol}$. This finding is not consistent with the results described above, which demonstrate that adding explicit waters can significantly alter the barrier heights, at least for waters added to the first hydration shell of a hydrophilic moiety such as a keto oxygen. I conclude that an implicit water environment is not capable of accurately assessing the energetics of this reaction, in which specific directional solvent-solute interactions, and also solventsolvent interactions in the vicinity of the solute, are important. It represents the barrier for fully optimized structures.

Incremental explicit water molecules placed in the manner described significantly stabilize all structures (reactants, transition states and products) with respect to infinitely separated species. Stabilization is generally greatest for the transition state, with the result that the reaction barrier decreases with increasing level of hydration. With five explicit water molecules, the forward reaction (diketone to ketoenol) is significantly lower. To make a physical connection, such structures might be thought of fully optimized and stable conformation that one might expect to see in a protective channel in an enzyme such as the major channel of CPO. This stable network of water molecules is present in 
the crystal structure reported by Kuehnel et. al. [38]. While this method does allow us to model a highly stable environment like the enzyme major channel of $\mathrm{CPO}$, it is not representative of kinetics in solution at $300 \mathrm{~K}$.

\begin{tabular}{|c|c|c|c|c|}
\hline & \multicolumn{2}{|c|}{ in vacuo } & \multicolumn{2}{c|}{ PCM $(\varepsilon=78.4)$} \\
\hline$\# \mathrm{H}_{2} \mathrm{O}^{\prime} \mathrm{s}$ & $\begin{array}{c}\Delta \mathrm{E} \\
(\mathrm{kcal} / \mathrm{mol})\end{array}$ & $\begin{array}{c}\Delta \mathrm{E}^{\mathrm{TS}, \mathrm{f}} \\
(\mathrm{kcal} / \mathrm{mol})\end{array}$ & $\begin{array}{c}\Delta \mathrm{E} \\
(\mathrm{kcal} / \mathrm{mol})\end{array}$ & $\begin{array}{c}\Delta \mathrm{E}^{\mathrm{TS}, \mathrm{f}} \\
(\mathrm{kcal} / \mathrm{mol})\end{array}$ \\
\hline 0 & -1.6 & 63.9 & -2 & 64.6 \\
\hline 1 & -3.1 & 27.7 & -6.3 & 26.8 \\
\hline 3 & -5.1 & 27.6 & -5.6 & \\
\hline 5 & -12.7 & $15.5(22)$ & -4.4 & 17.1 \\
\hline 6 & -11.6 & & & \\
\hline 8 & -17.2 & & -11.8 & \\
\hline 10 & -16.7 & & & \\
\hline 11 & -17.0 & & & \\
\hline
\end{tabular}

Table 17: Reaction energies and barrier heights ( $\mathrm{kcal} / \mathrm{mol})$ for reaction in water clusters 


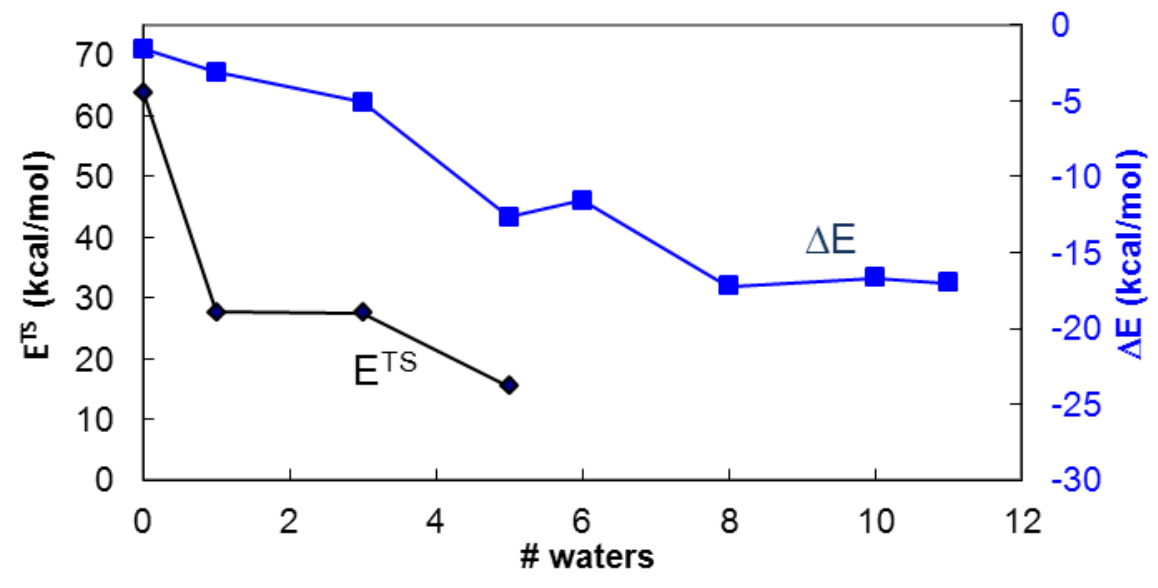

Figure 34: Reaction energies and barrier heights for reaction in water clusters

It is interesting to note that the water molecules significantly affect the barrier height only if they participate directly in a proton transfer, acting as a catalyst or if they help stabilize the catalytic water. For example, in the cluster with three waters only one water molecule is involved in the proton transfer and acts as a catalyst. The other two waters are only involved in hydration. The reaction barrier, $27.6 \mathrm{kcal} / \mathrm{mol}$, is almost the same as for the single-water example $(27.7 \mathrm{kcal} / \mathrm{mol})$. When two more water molecules were added in close vicinity to the catalytic water the barrier height dropped significantly. The reaction barrier dropped from $27.7 \mathrm{kcal} / \mathrm{mol}$ to $15.5 \mathrm{kcal} / \mathrm{mol}$.

I also used the IRC method $[58,59]$ to obtain the respective reactant and product structures from the transition states. The same reactant and product structures were obtained as before, except for the system with five water molecules. The IRC barrier is given in parentheses in Table 17 for the system with five water molecules. 


\subsection{Conclusions}

The calculations demonstrate that the barrier height is quite sensitive to the particular pattern of an elaborate hydrogen bonded structure. Thus for reaction at the active site of CPO, it is likely that the placement of water molecules, and possibly also charged side chains, is critical for achieving the lowest barrier to reaction. The barrier height is observed to be most sensitive to the placement of water molecules near the first catalytic water and less sensitive to waters located elsewhere. No proton transfer is observed from $\mathrm{HOCl}$ to $\mathrm{CPD}$ as might have been expected to initiate or accompany abstraction of the alpha hydrogen as $\mathrm{H}^{+}$.

The energies of reaction leveled off as the number of waters increased beyond five, but the barrier heights did not. Thus previously reported theoretical calculations on keto-enol tautomerization, which involved 1-4 waters, are unlikely to have achieved reaction barriers representative of solution conditions, which was in most cases the stated goal. More waters, and probably a different approach altogether, are needed to realistically model the barriers in full solvation conditions.

The location of transition states with many water molecules present is very difficult due to the high dimensional, flat potential energy surface. Therefore extending this approach to larger clusters was not feasible. It is emphasized that these structures are what one might expect to see in a protective channel in an enzyme such as the major channel of CPO and unlikely to be representative of liquid water at room temperature. In order to determine the barrier height representative of full solvation conditions, I employed a different approach, which is described in the next chapter. 


\section{STUDY OF CPD DIKETO $\rightarrow$ KETO-ENOL TAUTOMERIZATION UNDER FULL SOLVATION CONDITIONS}

To represent the solvated environment with explicit solvent molecules, I used Molecular Dynamics (MD) run at $300 \mathrm{~K}$ to obtain snapshots of reaction precursor structures. A QM/MM approach was then used to obtain an adiabatic reaction path and barrier height for each precursor structure. The replica path and Climbing Image Nudged Elastic Band (NEB) methods were used to determine the reaction paths.

\subsection{MD simulation and selection of frames}

For a reaction to occur in solution, the reactive molecules need to be favorably positioned and oriented with respect to each other. Such positioning, sometimes called a 'rare event' occurrence, is not likely to occur frequently on the nanosecond timescale. I therefore chose frames from the ten ns MD simulation that represent such 'rare event' occurrences. The MD provides the randomization of the water molecules present in a solvated environment.

When the $\mathrm{HOCl}$ and $\mathrm{CPD}$ approach each other in solution, they remain close for a duration on the order of a few ps, trapped by a solvent cage. I selected only one frame for each unique encounter, choosing frames at least ten ps apart. Ten ps represents the residence time for a hydrogen bond. Favorable conformations were selected such that the $\mathrm{HOCl}$ was near enough to $\mathrm{CPD}$ to form a hydrogen bond with one of the keto oxygens, 
and a water molecule was positioned ready to accept a proton from the alpha carbon of CPD and to donate a proton to the other keto oxygen. The selection process thus used the following criteria: The distance between one of the keto oxygens of CPD and the hydrogen of $\mathrm{HOCl}$ is less than or equal to $2.4 \AA$ (1). There exists a water molecule whose oxygen is within $2.4 \AA$ of the alpha carbon of CPD (2) and one of whose hydrogens is within $3.2 \AA$ of the keto oxygen not used in the first criterion (3). Statistics regarding the trajectory frames satisfying these criteria are shown in Figure 36. A representative selected structure is shown in Figure 35. From each unique encounter, the best frame was selected for further consideration. The best frame was taken as the one with the smallest value for criterion (1); in the case of a tie, the smallest value for criterion (2) was determining; and if a tie still remained, the smallest value for criterion (3) was decisive.

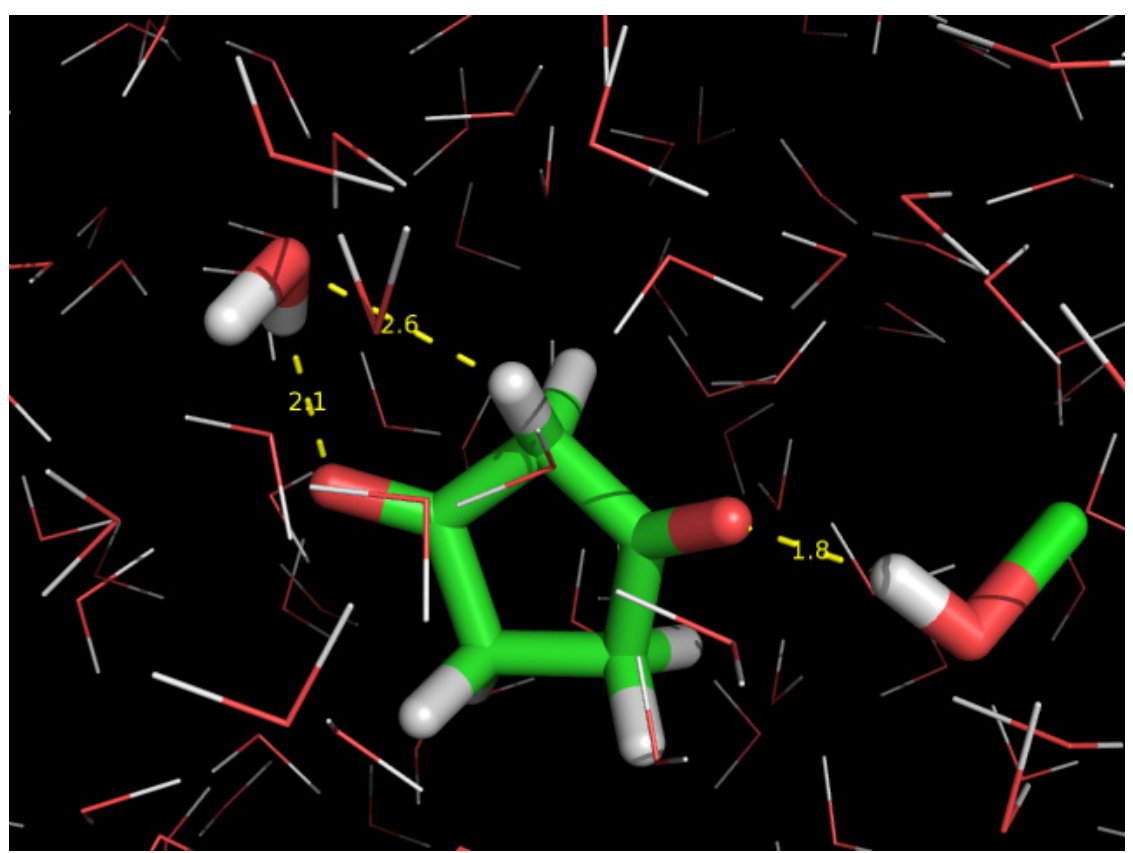

Figure 35: Representative rare event frame favorable for reaction, chosen from the MD simulation according to the selection criteria. Atoms treated at the QM level in subsequent QM/MM calculations are represented with thick sticks, and MM atoms are represented with thin lines 


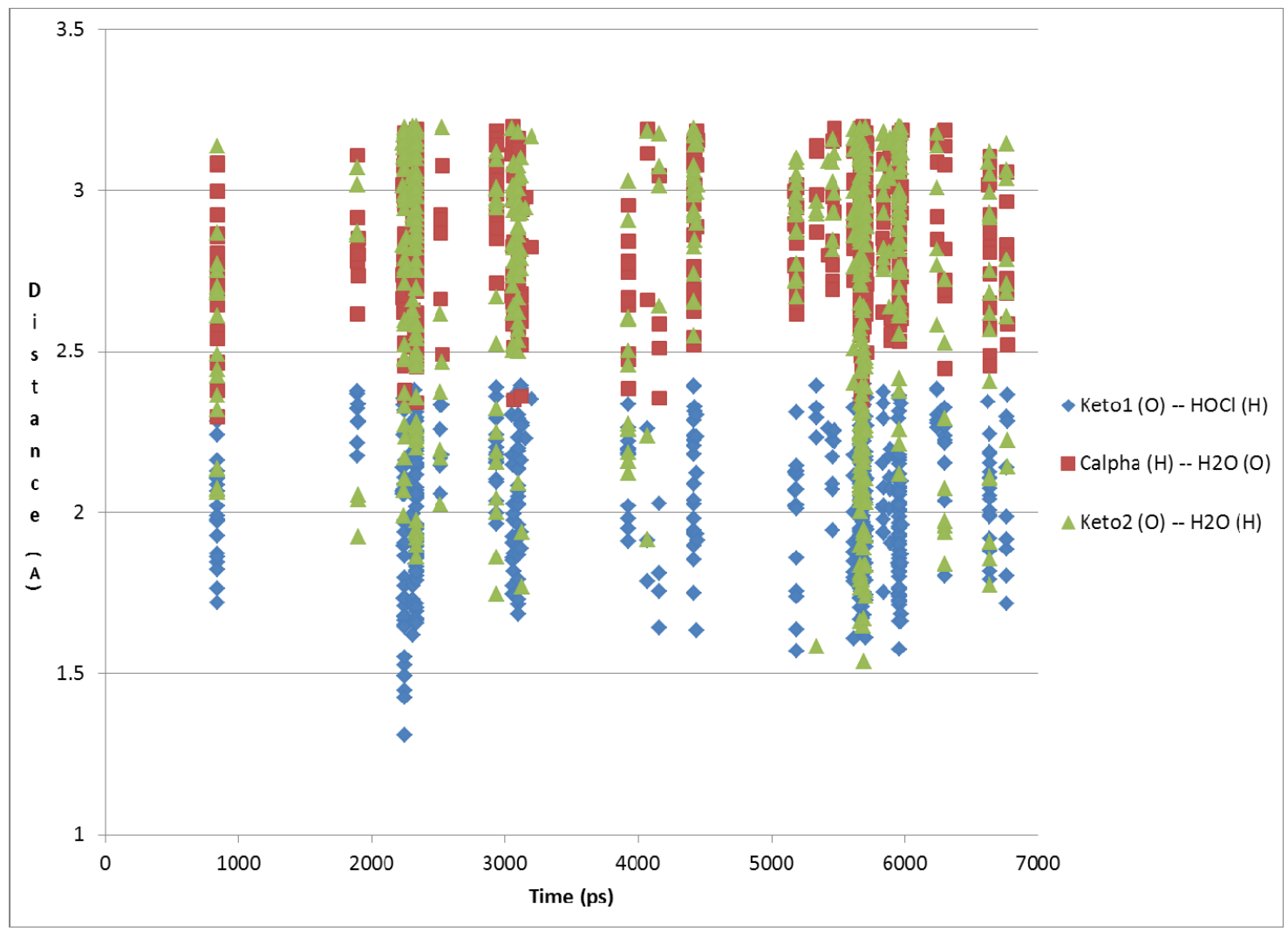

Figure 36: Trajectory frames satisfying the selection criteria. Each vertical group of data points represents one encounter, i.e., all frames lie within 10 ps of the best frame selected. Frames were saved every 20 steps (every $0.02 \mathrm{ps}$ ). 617 frames satisfied the selection criteria out of a total of 350,000 saved trajectory frames $(0.18 \%)$

\subsection{Determination of minimum energy path}

I determined the reaction barriers for the selected frames using a QM/MM model.

I treated the $\mathrm{CPD}, \mathrm{HOCl}$ and a single water molecule, which participates in the reaction catalytically, at the QM level and the rest of the water molecules at the MM level. All the MM waters were kept fixed while the $\mathrm{QM}$ atoms were flexible. Thus the barriers calculated are adiabatic with respect to the flexible degrees of freedom, in a fixed solvent environment representative of a liquid solution at $300 \mathrm{~K}$. Physically, this represents a 
reaction that takes place much faster than solvent response. This is clearly not what happens in reality but provides a well-defined limiting behavior for analysis. Each frame represents a different solvent environment consistent with a temperature of $300 \mathrm{~K}$. Boltzmann weighted averaging of barriers across a sufficient number of frames can provide an effective activation barrier for the limiting physical process considered. The MEP was determined with chain-of-replicas methods as described earlier.

\subsubsection{Determining MEP using Replica Path Method}

I used the Replica Path Method as the method of choice to map out the minimum energy path. In this method, a chain of conformations, or replicas, representing stages of reaction between reactants and products is created. The distance between pairs of conformations is defined using an RMSD metric. Simultaneous optimization of all replicas with a constraint to maintain spacing between the replicas yields an MEP. Optimization with the RPATh facility in CHARMM required 500 steps to achieve the convergence criterion. The convergence criterion was an average root mean square gradient (GRMS) value of less than $0.06 \mathrm{kcal} / \mathrm{mol} / \AA$ between successive 100 -cycle iterations. A typical optimization is shown in Figure 38. For frame 1 Table 18 shows the energy level convergences of the replicas for every 100 optimization cycle, Table 19 shows the gradient convergence criteria for every 100 optimization cycle and Table 20 shows that the RMSD deviations are within limit (i.e. less than 20\% RMSD deviation from neighboring replicas or images) to validate the parameters used in RPATH. For the 
optimizations, the end points were fixed. Consequently the minima, which correspond to reactants and products, occur at intermediate replicas. The reaction path obtained with RPATh results in approximately equal spacing between replicas on the PES. Thus the transition state is not located with precision. To obtain an accurate transition state, I refined the reaction path using the nudged elastic band method with the climbing image option (CI-NEB).

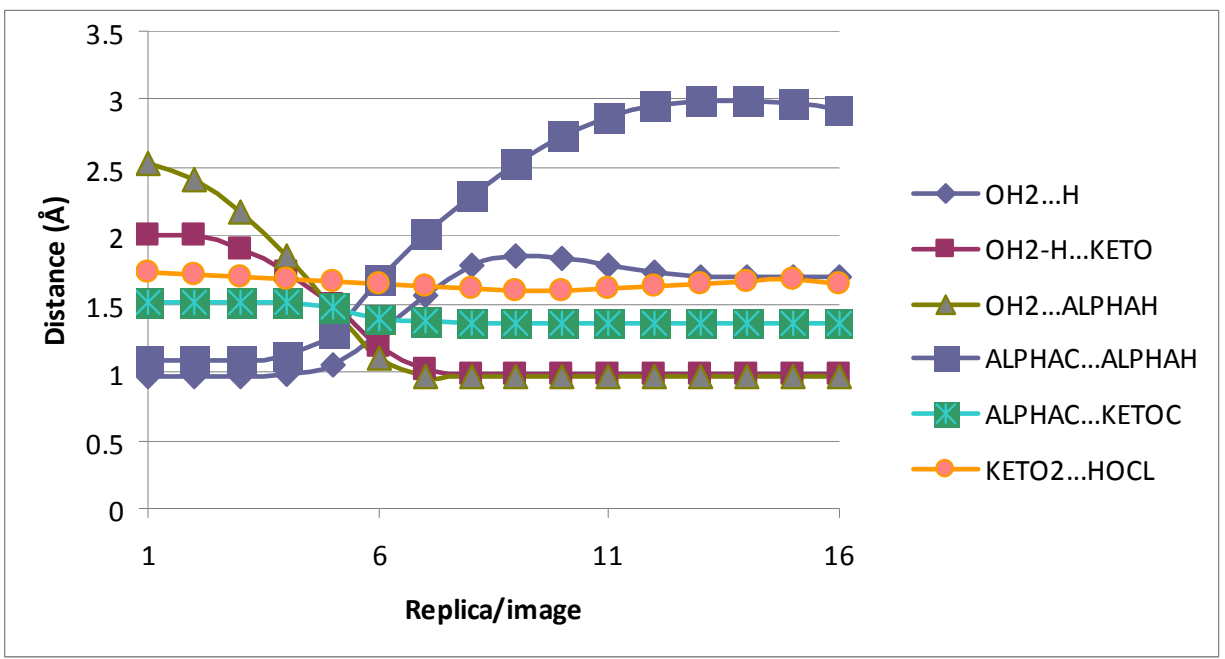

Figure 37: Frame 1: Distance change showing the hydrogen abstraction and enolization from reactant to product as the reaction proceeds in the vicinity of the transition state

\begin{tabular}{|c|c|c|c|c|c|}
\hline & \multicolumn{5}{|c|}{ Optimization steps (SD + ABNR) } \\
\hline Replicas/Images & $50+100$ & $50+200$ & $50+300$ & $50+400$ & $50+500$ \\
\hline 1 & 21 & 21 & 21 & 21 & 21 \\
\hline 2 & 2 & 2 & 2 & 1 & 1 \\
\hline
\end{tabular}




\begin{tabular}{|c|c|c|c|c|c|}
\hline 3 & 3 & 2 & 2 & 2 & 2 \\
\hline 4 & 4 & 4 & 6 & 9 & 7 \\
\hline 5 & 9 & 10 & 19 & 28 & 23 \\
\hline 6 & 20 & 26 & 30 & 18 & 22 \\
\hline 7 & 35 & 27 & 12 & 5 & 6 \\
\hline 8 & 21 & 12 & 5 & 2 & 2 \\
\hline 9 & 11 & 6 & 2 & 1 & 1 \\
\hline 10 & 7 & 4 & 2 & 1 & 1 \\
\hline 11 & 5 & 3 & 1 & 1 & 1 \\
\hline 12 & 3 & 3 & 1 & 1 & 1 \\
\hline 13 & 2 & 2 & 1 & 1 & 1 \\
\hline 14 & 1 & 1 & 0 & 0 & 0 \\
\hline 15 & 0 & 0 & 0 & 0 & 0 \\
\hline 16 & 15 & 15 & 15 & 15 & 15 \\
\hline
\end{tabular}

Table 18: Frame 1: Normalized energy level for each replica for every 100 ABNR optimization cycles. The maximum energy for each optimization cycle is highlighted 


\begin{tabular}{|c|c|}
\hline $\begin{array}{c}\text { Optimization Steps } \\
\text { (SD + ABNR) }\end{array}$ & $\begin{array}{c}\text { Avg. RMS } \\
\text { Gradient }\end{array}$ \\
\hline $50+100$ & 2.094 \\
\hline $50+200$ & 0.388 \\
\hline $50+300$ & 0.165 \\
\hline $50+400$ & 0.121 \\
\hline $50+500$ & 0.065 \\
\hline
\end{tabular}

Table 19: Frame 1: Average RMS Gradient for every 100 ABNR optimization steps

\begin{tabular}{|c|c|c|}
\hline Replicas/Images & RMSD & \%RMSD deviation \\
\hline 1 & 0.123 & $2.70 \%$ \\
\hline 2 & 0.122 & $3.70 \%$ \\
\hline 3 & 0.124 & $1.80 \%$ \\
\hline 4 & 0.13 & $3.00 \%$ \\
\hline 5 & 0.147 & $16.60 \%$ \\
\hline 6 & 0.13 & $3.30 \%$ \\
\hline
\end{tabular}




\begin{tabular}{|c|c|c|}
\hline 8 & 0.125 & $1.00 \%$ \\
\hline 9 & 0.125 & $1.00 \%$ \\
\hline 10 & 0.125 & $0.80 \%$ \\
\hline 11 & 0.124 & $1.50 \%$ \\
\hline 12 & 0.123 & $2.40 \%$ \\
\hline 13 & 0.123 & $2.30 \%$ \\
\hline 14 & 0.123 & $2.80 \%$ \\
\hline 15 & 0.123 & $2.80 \%$ \\
\hline
\end{tabular}

Table 20: Frame 1: RMSD and \% RMSD deviation from neighboring replicas

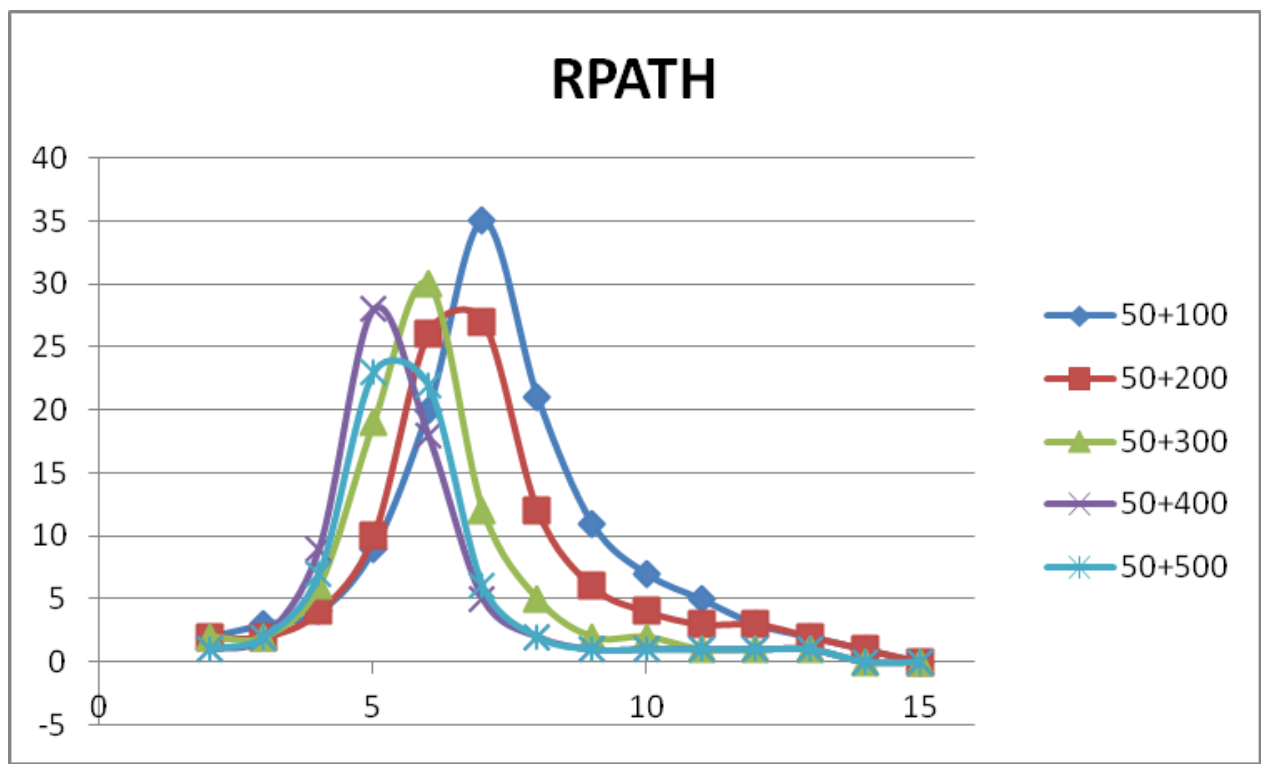

Figure 38: Optimization of Replica Path Method (SD+ABNR) 


\subsubsection{Locating transition state using Climbing Image Nudged Elastic Band}

I use CI-NEB to locate the transition state once I have the MEP mapped out by the Replica Path Method. This option causes a path point near the transition state to climb in energy toward the transition state, while all other path points are energy minimized in directions orthogonal to the MEP. A typical optimization is shown in Figure 39. Further details are given in the supplemental information.

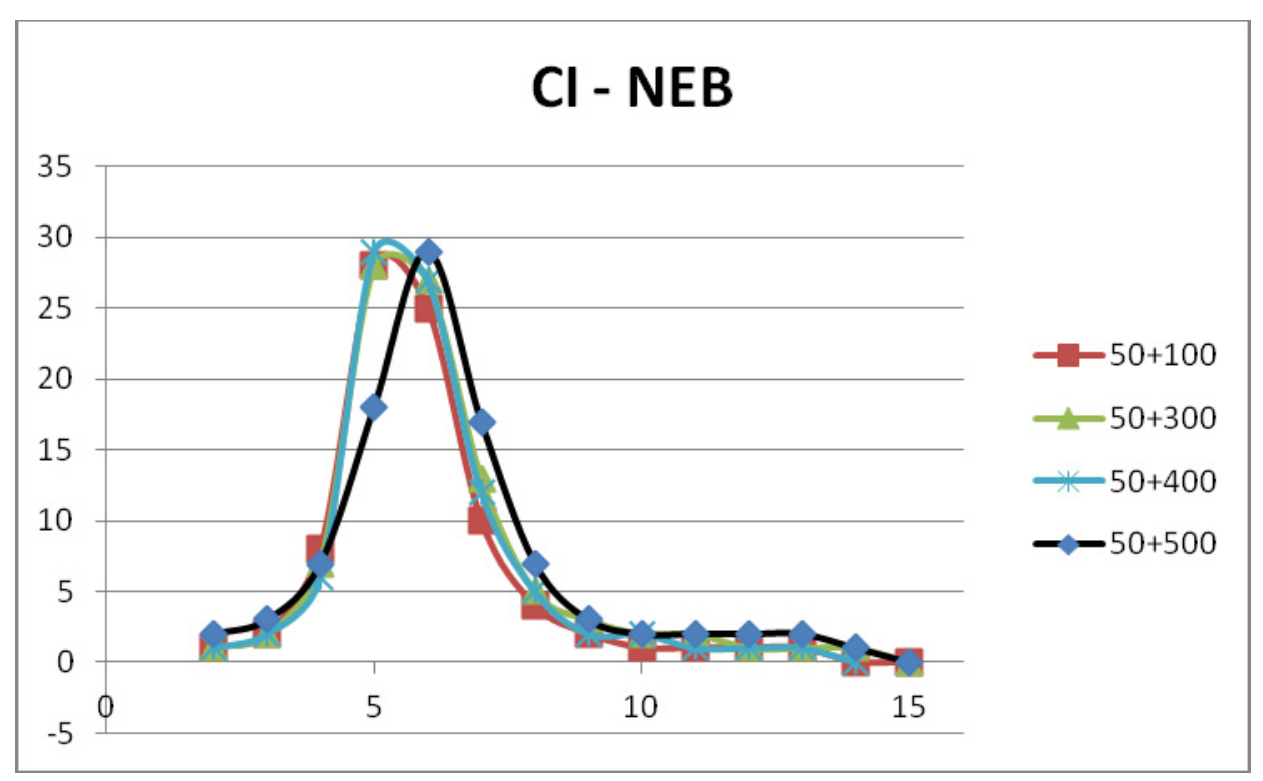

Figure 39: Optimization of CI-NEB to locate Transition state (SD+ABNR)

Table 21 gives the barrier height for each of the selected frames. There is a substantial variability in barrier height of $25-44 \mathrm{kcal} / \mathrm{mol}$. Applying Boltzmann weighting to the barrier heights for the ten frames in Table 21, I obtain an effective activation energy $\left(E_{a}\right)$ of $25 \mathrm{kcal} / \mathrm{mol}$. Some important distances are tabulated in $\mathrm{x}, \mathrm{y}$, and table for reactant transition state and product respectively. To investigate the cause of the variation 
in barrier heights, I visually analyzed each frame, focusing on hydrogen-bonding interactions likely to be important for reactants and transition states. These are summarized in Table 25.

\begin{tabular}{|c|c|}
\hline Frame & $\Delta \mathrm{E}$ \\
\hline 1 & 27 \\
\hline 2 & 30 \\
\hline 3 & 36 \\
\hline 4 & 41 \\
\hline 5 & 44 \\
\hline 6 & 42 \\
\hline 7 & 35 \\
\hline 8 & 38 \\
\hline 10 & 25 \\
\hline
\end{tabular}

Table 21: Barrier heights (kcal/mol) for reaction for selected frames from solvated MD simulation 


\begin{tabular}{|c|c|c|c|c|c|c|}
\hline Frame & $\begin{array}{c}\text { OH2 } \\
\ldots\end{array}$ & $\begin{array}{c}\text { OH2-H } \\
\ldots\end{array}$ & $\begin{array}{c}\text { OH2 } \\
\ldots\end{array}$ & $\begin{array}{c}\text { ALPHAC } \\
\ldots\end{array}$ & $\begin{array}{c}\text { ALPHAC } \\
\ldots\end{array}$ & $\begin{array}{c}\text { KETO2 } \\
\ldots\end{array}$ \\
\hline 1 & 0.972 & 1.995 & 2.379 & 1.093 & 1.512 & 1.721 \\
\hline 2 & 0.968 & 2.823 & 3.201 & 1.100 & 1.522 & 1.792 \\
\hline 3 & 0.974 & 2.107 & 2.490 & 1.099 & 1.521 & 1.675 \\
\hline 4 & 0.974 & 2.104 & 2.675 & 1.095 & 1.522 & 1.942 \\
\hline 5 & 0.979 & 2.385 & 3.165 & 1.098 & 1.501 & 2.321 \\
\hline 6 & 0.972 & 2.651 & 2.915 & 1.096 & 1.523 & 1.786 \\
\hline 7 & 0.973 & 2.201 & 2.517 & 1.092 & 1.511 & 2.024 \\
\hline 8 & 0.97 & 3.42 & 2.22 & 1.10 & 1.52 & 1.72 \\
\hline 10 & 0.972 & 1.796 & 2.247 & 1.096 & 1.526 & 1.714 \\
\hline
\end{tabular}

Table 22: Reactant Distances for all the frames

\begin{tabular}{|c|c|c|c|c|c|c|}
\hline Frame & $\begin{array}{c}\text { OH2 } \\
\ldots\end{array}$ & $\begin{array}{c}\text { OH2-H } \\
\ldots\end{array}$ & $\begin{array}{c}\text { OH2 } \\
\ldots \\
\text { KETO }\end{array}$ & $\begin{array}{c}\text { ALPHAC } \\
\ldots\end{array}$ & $\begin{array}{c}\text { ALPHAC } \\
\ldots\end{array}$ & $\begin{array}{c}\text { KETO2 } \\
\ldots \\
\text { AOCL }\end{array}$ \\
\hline 1 & 1.11 & 1.38 & 1.22 & 1.47 & 1.44 & 1.64 \\
\hline 2 & 1.13 & 1.39 & 1.28 & 1.53 & 1.44 & 1.64 \\
\hline
\end{tabular}




\begin{tabular}{|c|c|c|c|c|c|c|}
\hline 3 & 1.14 & 1.34 & 1.23 & 1.49 & 1.44 & 1.71 \\
\hline 4 & 1.24 & 1.23 & 1.27 & 1.47 & 1.44 & 1.95 \\
\hline 5 & 1.15 & 1.41 & 1.26 & 1.66 & 1.41 & 3.01 \\
\hline 6 & 1.2 & 1.26 & 1.28 & 1.47 & 1.44 & 1.65 \\
\hline 7 & 1.18 & 1.3 & 1.22 & 1.47 & 1.43 & 1.92 \\
\hline 8 & 1.11 & 1.42 & 1.23 & 1.54 & 1.44 & 1.73 \\
\hline 9 & 1.02 & 1.67 & 1.19 & 1.52 & 1.44 & 1.77 \\
\hline 10 & 1.16 & 1.33 & 1.27 & 1.5 & 1.45 & 1.66 \\
\hline
\end{tabular}

Table 23: Transition state Distances for all the frames

\begin{tabular}{|c|c|c|c|c|c|c|}
\hline Frame & $\begin{array}{c}\mathbf{O H} 2 \\
\ldots \\
\mathbf{H}\end{array}$ & $\begin{array}{c}\mathbf{O H} 2-\mathbf{H} \\
\ldots \\
\text { KETO }\end{array}$ & $\begin{array}{c}\mathbf{O H} 2 \\
\ldots \\
\text { ALPHAH }\end{array}$ & $\begin{array}{c}\text { ALPHAC } \\
\ldots \\
\text { ALPHAH }\end{array}$ & $\begin{array}{c}\text { ALPHAC } \\
\ldots \\
\text { KETOC }\end{array}$ & $\begin{array}{c}\text { KETO2 } \\
\ldots \\
\mathbf{H O C L}\end{array}$ \\
\hline 1 & 1.71 & 0.99 & 0.97 & 2.93 & 1.37 & 1.66 \\
\hline 2 & 1.69 & 0.99 & 0.96 & 2.48 & 1.37 & 1.61 \\
\hline 3 & 2.00 & 0.98 & 0.97 & 3.07 & 1.36 & 1.66 \\
\hline 5 & 3.18 & 0.97 & 0.97 & 3.29 & 1.35 & 1.82 \\
\hline 5 & 2.26 & 0.98 & 0.97 & 3.16 & 1.35 & 2.66 \\
\hline
\end{tabular}




\begin{tabular}{|c|c|c|c|c|c|c|}
\hline 6 & 1.94 & 0.98 & 0.97 & 3.07 & 1.36 & 1.63 \\
\hline 7 & 3.48 & 0.98 & 0.97 & 2.51 & 1.36 & 1.87 \\
\hline 8 & 4.04 & 0.97 & 0.97 & 3.00 & 1.36 & 1.68 \\
\hline 9 & 1.74 & 0.99 & 0.97 & 2.76 & 1.36 & 1.77 \\
\hline 10 & 1.95 & 0.98 & 0.97 & 4.62 & 1.37 & 1.59 \\
\hline
\end{tabular}

Table 24: Product Distances for all the frames

\begin{tabular}{|c|c|c|c|c|c|c|}
\hline & \multicolumn{2}{|c|}{ Frame 1 } & \multicolumn{2}{c|}{ Frame 9 } & \multicolumn{2}{c|}{ Frame6 } \\
\cline { 2 - 7 } & Reactant & TS & Reactant & TS & Reactant & TS \\
\hline HBond & 1 & 1 & 0 & 0 & 1 & 2 \\
\hline RMSD & 0.07 & 0.21 & 0.38 & 0.30 & 0.29 & 0.52 \\
\hline
\end{tabular}

Table 25: Important hydrogen bonding interactions for reactants, transition states and products calculated for selected frames

To gain further insight into the cause of the variability in barrier heights, I recalculated the MEP, allowing nearby waters to respond to the progress of reaction. The MM water molecules within $2.6 \AA$ of the $\mathrm{QM}$ atoms were made flexible, with the remaining MM waters still fixed. Distinguishing the treatment of water molecules in this way is easily achieved in the replica path implementation in CHARMM, as it allows for 
the replicated system to be larger than the QM system. When this test was performed on one of the frames with a high barrier, frame 6 , the barrier height decreased by $7 \mathrm{kcal} / \mathrm{mol}$. The flexible MM water molecules underwent significant motion to stabilize the transition state structure, but little motion in the reactant structure, which was already solvent stabilized. When the test was performed on low-barrier structures, frames 1 and 9 , there was little change in the barrier height.

The hydrogen bond analysis parameters had a cutoff distance of $4.0 \AA$ and an angle of 150.0 degrees between acceptor and donor atoms. Only hydrogen bonds between the QM solvation shell and the $\mathrm{CPD}, \mathrm{HOCl}$ and the catalytic water were analyzed.

The RMSD was calculated for the flexible solvation shell with respect to the fixed shell in the reactant and transition state structures for frames 1,9 and 6.

Returning to the original reaction barrier calculations without the additional solvent flexibility, I can now trace the variability in barrier heights to the degree to which surrounding solvent waters stabilize the reactants. In no cases were transition states stabilized, but in some reactants were. In reality, it is unlikely that relatively massive solvent oxygens can move on the time scale of the proton transfer processes. Thus solvent stabilization of transition states is unlikely to occur, and the original set of barrier height calculations is more representative of the actual physical process than the calculations in which surrounding waters are allowed to move during the proton transfer processes. 


\subsection{Conclusions}

I find that the solvating water environment creates a wide range of barriers for potentially reactive events. In my sampling of ten frames, these range from 25 to 44 $\mathrm{kcal} / \mathrm{mol}$. In view of Boltzmann weighting, the lowest of these are dominant and the effective barrier is $26 \mathrm{kcal} / \mathrm{mol}$, which compares with the barrier of $28 \mathrm{kcal} / \mathrm{mol}$ for gasphase reaction with a single catalytic water. Thus, surprisingly, the barrier in a fully solvated system and the barrier with only a single water present are nearly the same. Close analysis reveals that this is not because other waters do not play a significant role in reaction. Rather it is because of a balancing effect. In low-barrier reaction events, which are mainly responsible for the observed rate constant, surrounding waters stabilize the transition state and the reactants to nearly the same extent. This is not true of most potentially reactive encounters; more often, surrounding waters stabilize reactants to a greater extent. Assuming that proton transfer occurs faster than water molecules can reorient, those same solvating waters are usually ill-positioned to stabilize the transition state and thus have a net effect of retarding rather than promoting reaction. In a small percentage of cases, though, the surrounding waters are positioned so as to lower the energies of both reactants and the transition state. Thus I find that transition state stabilization by solvent water is critical for reaction in solution. Transition state stabilization is also thought to be an important mode via which enzymes catalyze reactions. Thus it appears that enzymes and aqueous solution may have similar ways of catalyzing keto-enol tautomerization. 


\section{OVERALL CONCLUSIONS}

The goal of this work was to elucidate the chlorination mechanism of chloroperoxidase using CPD as a model substrate, and to shed light on which of two possible reaction pathways is more likely: reaction with free $\mathrm{HOCl}$ in aqueous solution or reaction at the enzyme active site. I show that the rate limiting step for the chlorination of CPD is the diketo-ketoenol tautomerization step and that the solvent environment plays a crucial role in the reaction. I model the hydration environments of the two possible pathways and calculate the reaction barriers for both. I observe that a limited and optimized (active-site-like) hydration environment leads to a lower energetic barrier than the fully solvated model representative of the aqueous environment at room temperature. This result indicates that the stable water network near the active site and the access channel is likely to facilitate the chlorination mechanism. Confirmation of this predication could be obtained from calculations of the reaction barrier with the enzyme active site explicitly modeled, including active site residues and hydrating waters, using a QM/MM approach. This, however, is beyond the scope of the current project.

I determined the nature of the influence of the solvent environment on the reaction barrier. I observe that stabilization of the catalytic water by other solvent molecules is critical for keto-enol tautomerization. When studying a limited-hydration environment, placement of water molecules is more important than the number of water molecules. Careful placement of hydrating water molecules saves a significant amount of computational time in such studies. Previous theoretical limited-hydration studies have been accepted as a tractable method for determining effective reaction barriers in 
solution. My work demonstrates that the limited-hydration approach is inadequate for determining effective reaction barriers for keto-enol tautomerization. In particular, I demonstrate that previous theoretical studies of keto-enol tautomerization in a limitedhydration environment, which used five or fewer waters, are not representative of a solution environment. My modeling of the reaction in a fully-solvating environment demonstrates that reaction proceeds when the instantaneous dynamical water environment is close to optimal for stabilizing the transition state. 


\section{OTHER SYSTEMS STUDIED/CONTRIBUTED TO}

This chapter describes my contributions to three other projects carried out in the Chatfield research group. Section 7.1 describes work on dehaloperoxidase, an enzyme bearing similarity to chloroperoxidase, the subject of my main project. Section 7.2 contains the preliminary results of an MD study of the solution dynamics of a small, highly cross-linked peptide, $\alpha$-conotoxin GI. Section 7.3 contains work done on the CPOcatalyzed epoxidation reaction. My major contribution to the studies described in sections 7.1 and 7.3 was the development of parameters for molecular mechanics modeling, including MD simulation. This work was a natural application of the expertise I gained in parameter development working on parameters for molecules involved in the CPOcatalyzed halogenation reaction, as explained in chapter 3. My contribution to the project described in section 7.1 also involved carrying out MD simulations using techniques and scripts I developed for my main project. The entire project described in section 7.2 was carried out primarily by me. This work in section 7.2 was a preliminary project carried out before beginning the CPO-catalyzed halogenation work that is focus of this dissertation. In carrying out the work described in section 7.2, I became familiar with molecular modeling techniques that I was able to apply to the other system studied. My contribution to each project is detailed in the subsequent sections. 


\section{1. $D H P$}

I made contributions to an MD study of the enzyme dehaloperoxidase (DHP), which is found in the marine worm Amphitrite ornata. The enzyme has a globin fold and can bind dioxygen as can most globins, but its primary function is to catalyze the dehalogenation of p-halophenols and related compounds. Thus DHP can function either as a dehalogenase or as a globin. The mechanism via which the enzyme switches between the two modalities is of interest. An active-site residue, His55, which can adopt multiple conformations, has been thought to play a major role in the switching between modalities. Molecular dynamics simulations of wildtype DHP and two mutants, H55V, H55R, with and without a p-iodophenol ligand present, were performed.

My contribution to this project was development of parameters for the piodophenol ligand, much of the simulation setup, and the running of several of the native and mutated enzyme simulations. Project design and analysis of the results were carried out by other members of the research group. A very brief summary of the simulation method and analysis to date is described in the next paragraph.

A 10-ns simulation was performed and analyzed for each system. Hydrogen bonds between the ligand, Tyr38 and His55 were found to highly stabilize the ligand at the active site. The ligand was found to block access to the heme iron, for most of the 10ns simulation. When His55 was mutated to Val or Arg, the p-iodophenol ligand was no longer maintained in a position where it blocks access to the heme iron. The simulations also provided insight into the external binding site of the substrate. 


\section{2. $\alpha$-Conotoxin $G I$}

I began work on this project at the outset of my Ph.D. The project was framed as a joint MD/NMR study of motional motifs, with an ultimate goal of contributing to the development of MD-based methods for interpreting the results of dynamical NMR. For practical reasons relating to difficulties in obtaining the labeled peptide for the NMR portion of the study, the project was not completed.

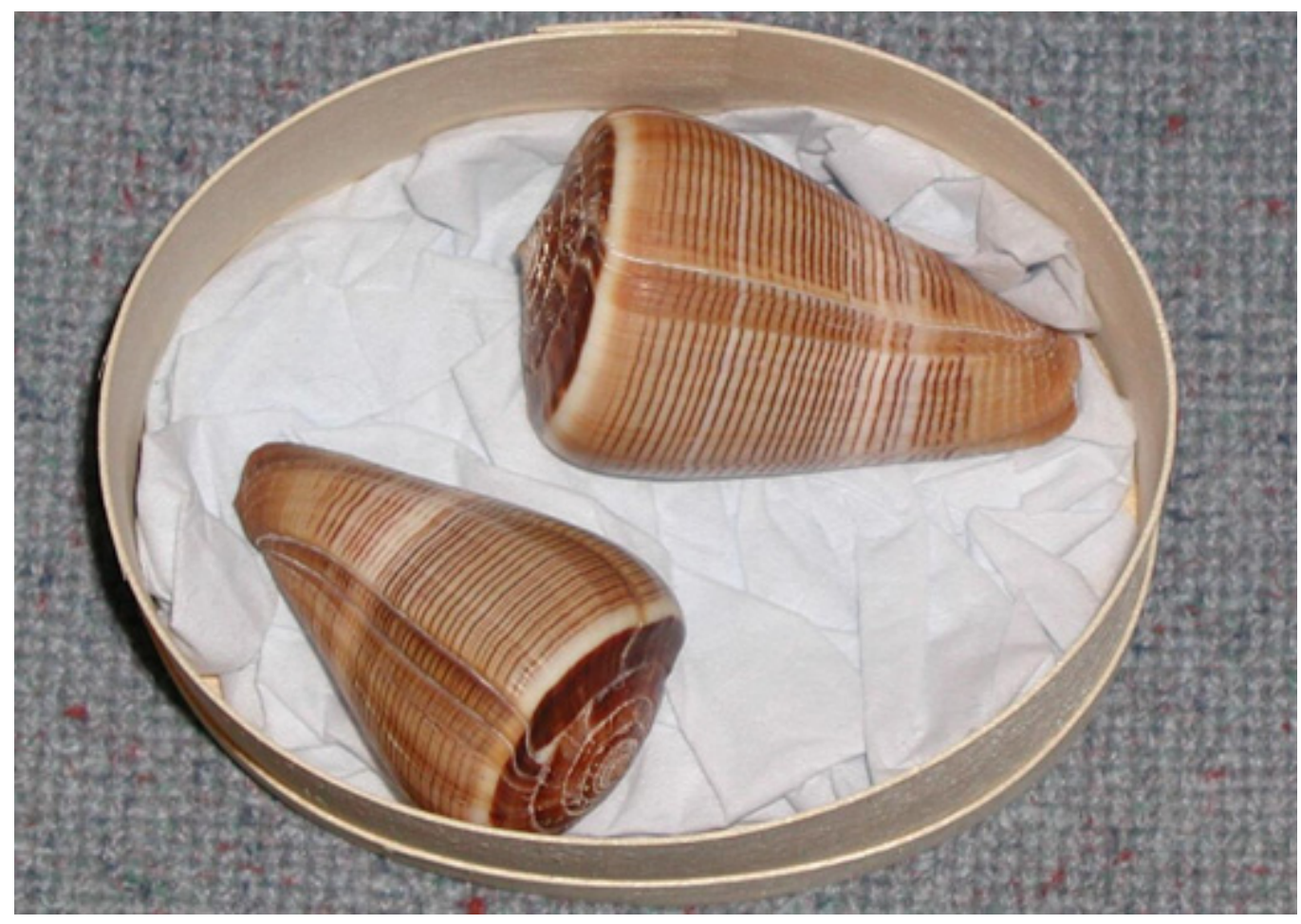

Figure 40: Cone snail

The peptide $\alpha$-conotoxin GI belongs to a family of small multiply cross-linked peptide toxins produced by marine mollusks known as cone snails. The slow-moving snails use the toxins to hunt their prey, which are usually fish. Its venom needs to act fast 
in order to bring down its prey given the snails' limited mobility in comparison to its prey, which is one of the reasons its venom has evolved to be highly potent. Depending on the species, the conotoxins range in length from 13-30 residues, making them among the smallest of peptide toxins (snake venom toxins, by comparison, tend to be in the 70residue range). Conotoxins act on the nervous system, serving to paralyze the snails' prey. For example, the $\alpha$-conotoxins bind to the nicotinic acetylcholine receptor (nAChR), to Ca-gated voltage channels.

Conotoxins are notable for their specificity of binding. For example, the $\alpha$ conotoxins of one species of cone snail are often inactive against the nAChR of the prey of another species of cone snail. The specificity of conotoxins are achieved through conservation of a scaffold based on patterns of cross-linking combined with a remarkable variability of loop composition. For this reason, conotoxins are of interest as possible lead compounds for drug development. The $\alpha$-conotoxins are of particular interest because their binding properties may help to map out the nAChR, whose threedimensional structure is thus far unknown.

The $\alpha$-conotoxin GI was one of the first-isolated conotoxins and has been widely studied. Its sequence is ECCNPACGRHYSC, and there are disulfide bonds between residues 2 and 7, and 3 and 13. The three-dimensional structure has been characterized by X-ray crystallography and solution-state NMR. Two regions of secondary structure have been identified. In all structures, residues 4-8 have been described as 3-10 helical. Residues 9-13 have been described as a $\beta$-turn. In the case of the X-ray structure, this was on the basis of both backbone dihedral angles and hydrogen bonds. For the NMR structures, secondary structure assignment was based mainly on dihedral angles. 


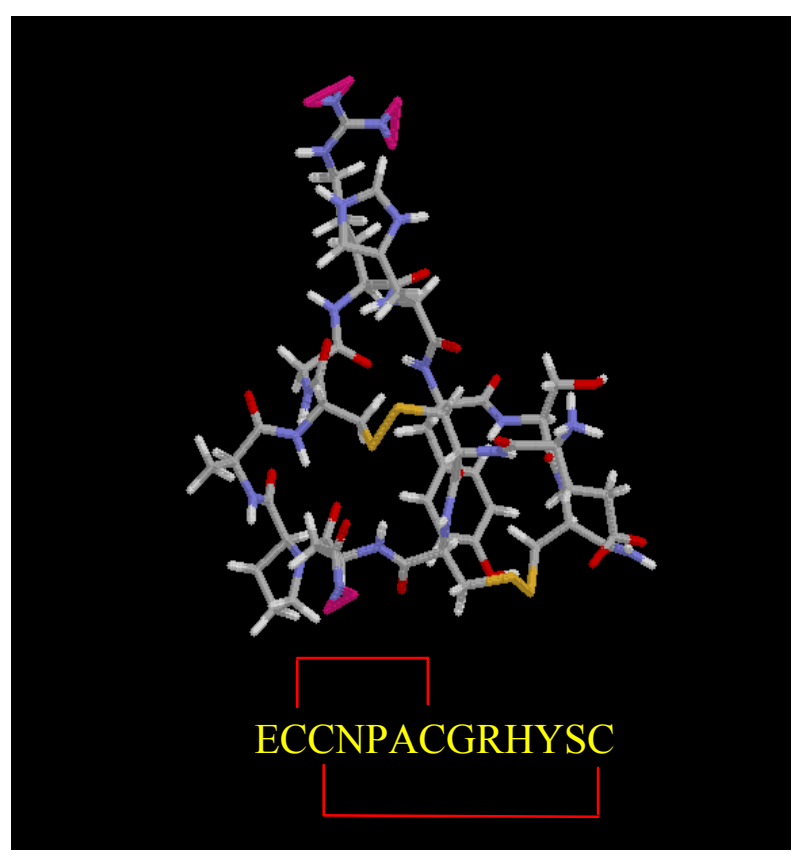

Figure 41: $\alpha$-conotoxin GI

Notably, a recent NMR study identified two distinct conformers present in a 70:30 ratio (referred to hereafter as the major and minor conformers) on the basis of simulated annealing with the NMR structural restraints. It may be important to understand whether the conformers interconvert and whether one or both is active in binding to $\mathrm{nAChR}$.

Multiple molecular dynamics simulations were performed on a 13-residue, doubly cross-linked peptide toxin, $\alpha$-conotoxin GI, in each of two solution conformations identified by NMR. The two conformers remained distinct on the 100-ns simulation time scale, supporting the hypothesis that only one of the conformers is biologically active. The secondary structure as judged by backbone dihedral angles was preserved during simulation, but backbone hydrogen bonds were almost entirely absent. This observation suggests that binding at the active site probably involves hydrogen bonds to the peptide 
backbone. The backbone exhibited unusual, concerted motions of dihedral angles in nonconsecutive residues, which may have implications for the binding free energy.

Below is a set of figures that bring out the salient features of the simulation. Figure 42 shows overlays of the several structures obtained from NMR structural data on peptide in solution using simulated annealing. The backbone and most sided chains have well defined conformations, but several side chains are clearly mobile. From this structure, two primary backbone conformations were determined. These are shown in Figure 43 and are called the major and minor structures. Figure 44 depicts the root mean square deviation (RMSD) in the backbone coordinates for ten 10-ns simulations of each structure (major and minor). The RMSD for both structures are shown together on each panel. It can be seen that the structures remain distinct on the 10-100 ns time scale.

Figure 45 depicts correlation functions, $C_{i}(t)$, for motion of backbone N-H bond vectors for all residues of each of the two structures. The correlation functions are defined by

$$
C_{i}(t)=\left\langleP _ { 2 } \left( a_{i}(0) \cdot\left(a_{i}(t)\right\rangle\right.\right.
$$

where $f_{2}=\frac{R}{2} x^{2}-\frac{1}{8}$ is a second Legendre polynomial and $\hat{u}_{i}$ is a unit vector in the direction of the $\mathrm{N}-\mathrm{H}$ bond vector. The asymptotic value of $C_{i}(t)$ as time gets large is the order parameter, $\mathrm{S}^{2}$. Small values of $\mathrm{S}^{2}$ correspond to relatively unrestricted motion, and values of $S^{2}$ close to one correspond to highly restricted mobility. The two sets of data (major and minor) have very different asymptotic values, but this is because the data were treated differently. The trajectory frames for the major structures were reoriented 
before calculating the correlation functions, while those for the minor structures were not. When the trajectory frames for the minor structures are reoriented to remove overall translation and rotation (data not shown), the asymptotic behavior of the correlation functions is very similar to what is seen for the major structures in Figure 45. Values of $S^{2}$ by residue are depicted with a color code for both structures and also for the crystal structure (obtained from X-ray B factors) in Figure 46. The crystal structure shows generally more motional restriction (larger average value of $\mathrm{S}^{2}$ ) as a consequence of crystal packing; the simulation environment is aqueous solution.

Maps showing the pattern of $\mathrm{N}-\mathrm{H}$ backbone bond vector motion are shown in Figure 47. These are a major finding of the work. The N-H bond vectors display differing modes of motion, with some exhibiting motion within a cone, others elongated mobility, and yet others bimodal sampling. This indicates that interpretation of NMRderived order parameters in terms of motional models must be made with care.

Finally, Figure 48 shows that some backbone dihedrals angles exhibit correlated motions. When interpreting order parameters in terms of conformational entropy, this correlated motion will need to be taken into account. 


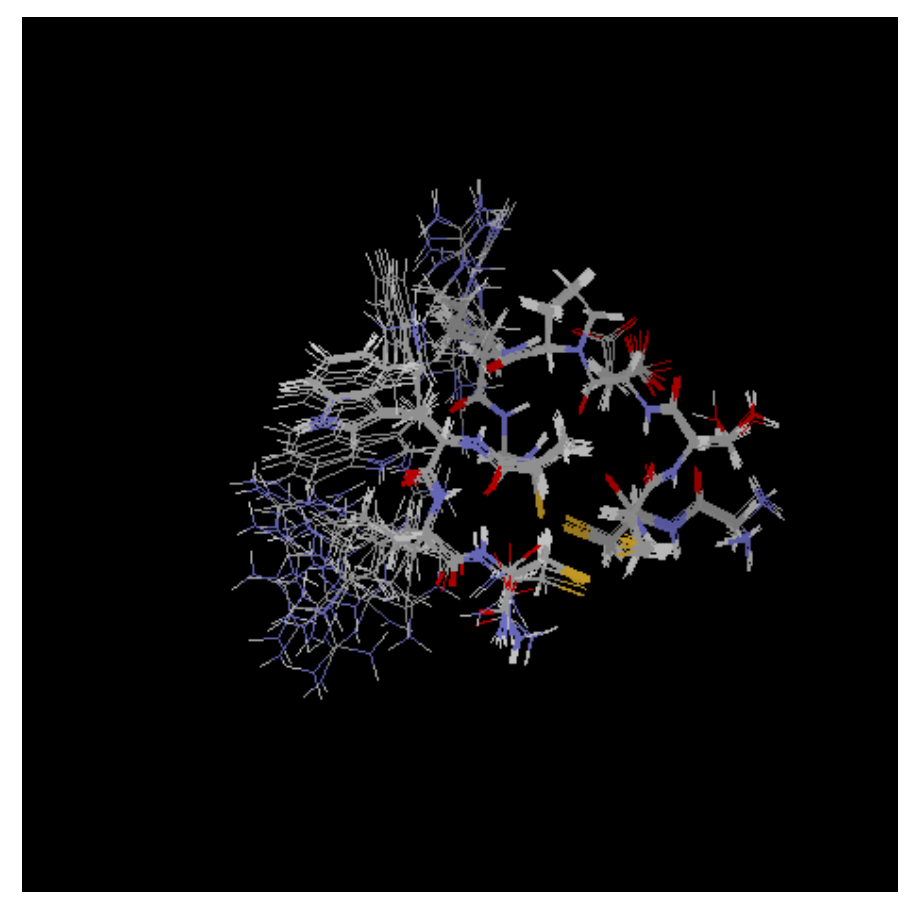

Figure 42: NMR structure showing stable backbone

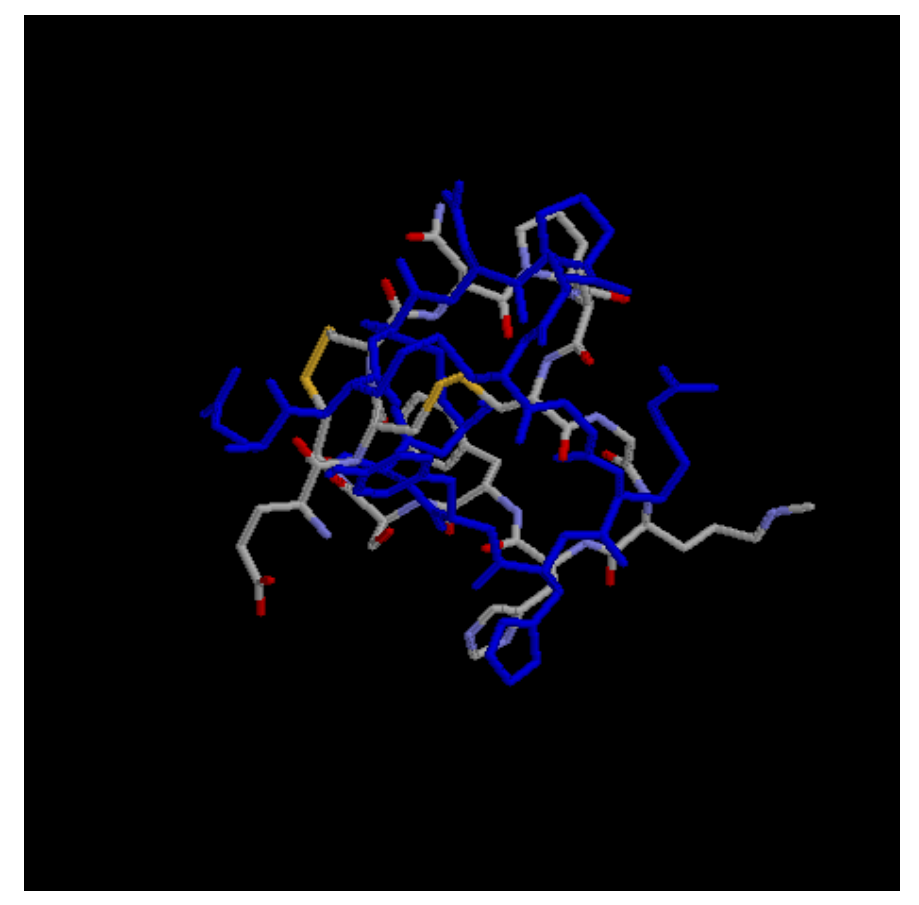

Figure 43: distinct structures in solution - Major and Minor 

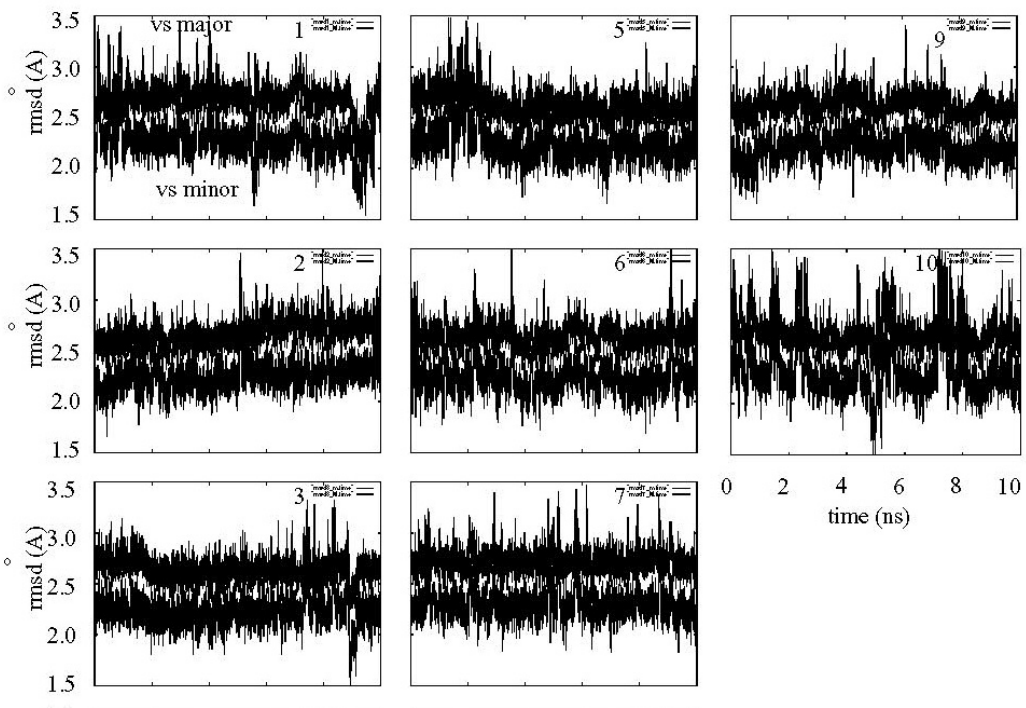

time (ns)
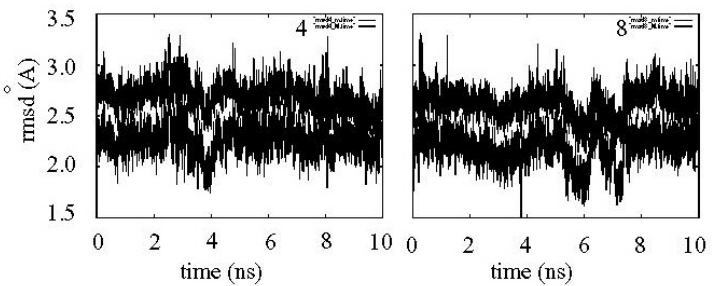

Figure 44: Simulation of major and minor conformation for 10x10 ns simulation demonstrating that the two structures are stable and distinct 

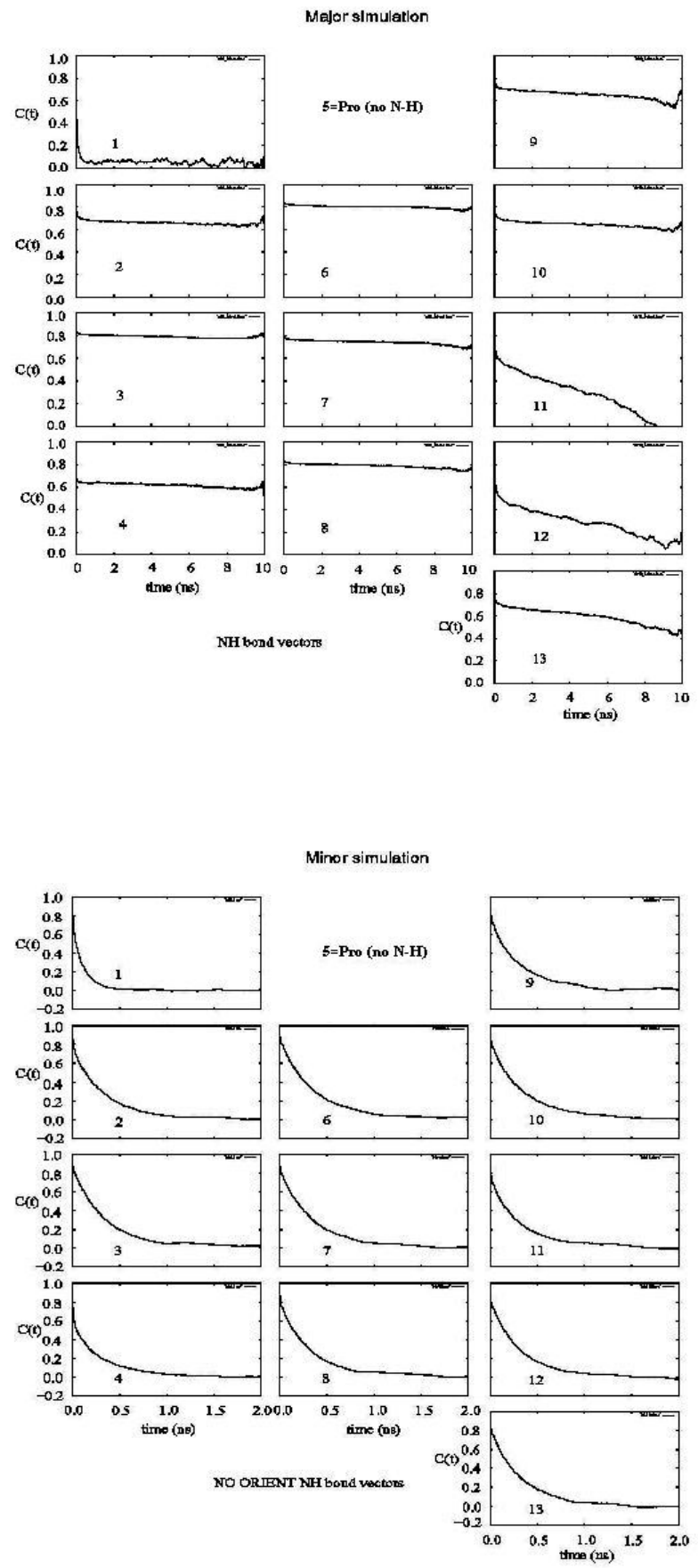

Figure 45: N-H correlation functions. Trajectory frames were reoriented for Major conformation but not for Minor conformation ( $S^{2}$ values shown elsewhere are based on reoriented data.) 


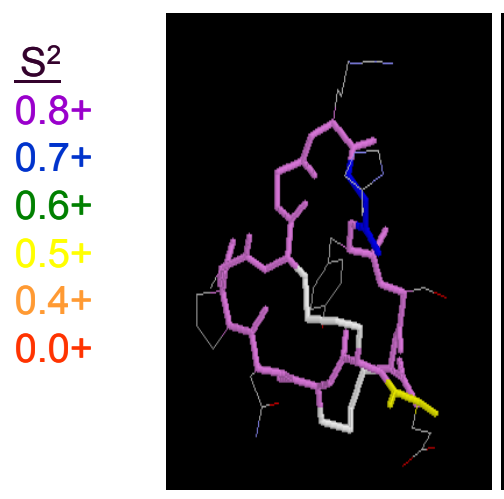

Crystal $\overline{S^{2}}=0.84$

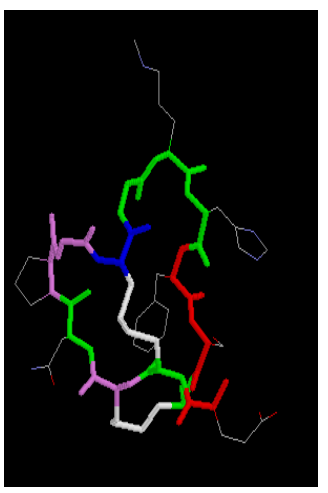

Major $\overline{S^{2}}=0.64$

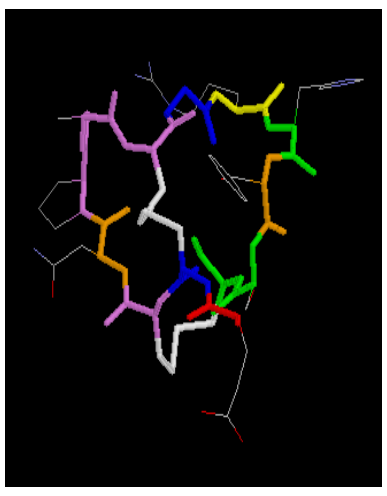

Minor $\overline{S^{2}}=0.66$

Figure 46: Backbone NH order parameter

Crystal
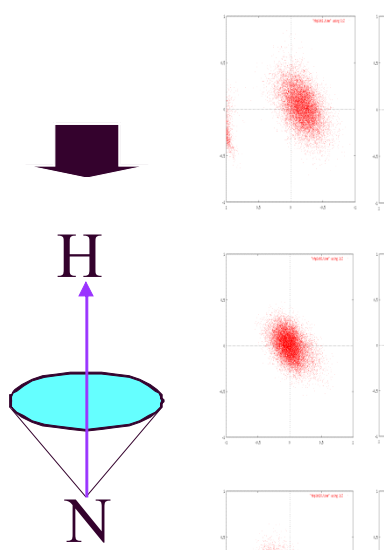
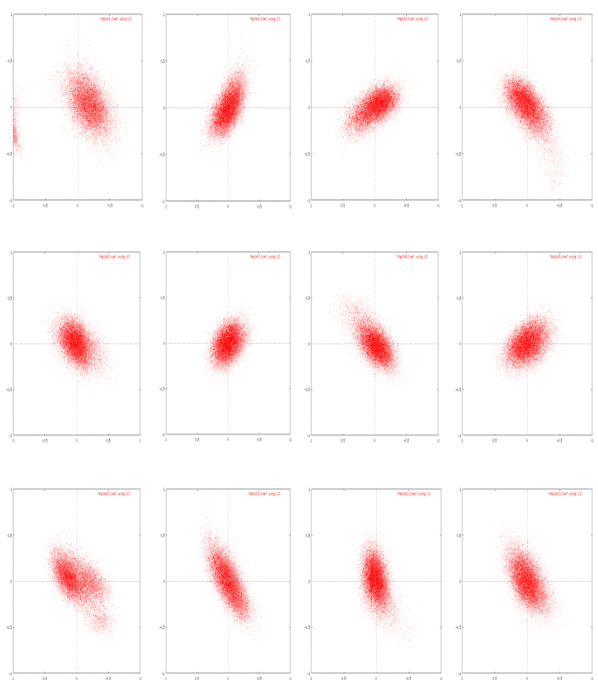

Solution (Major)
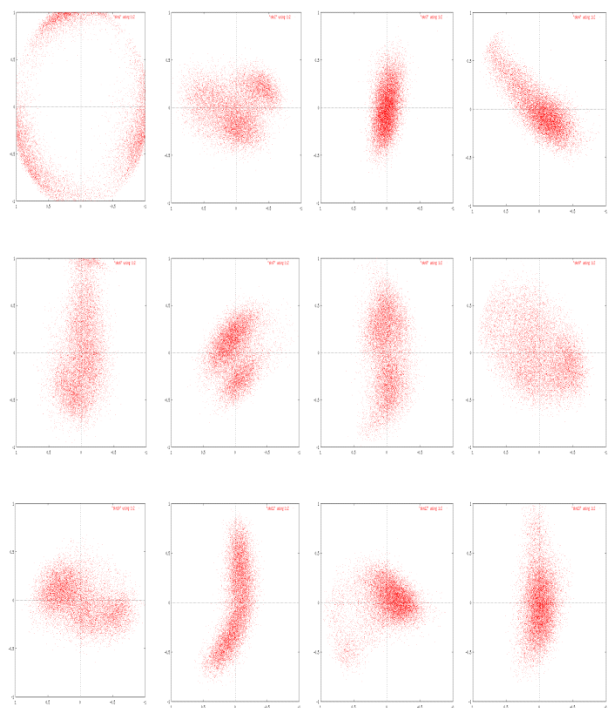

Figure 47: NH bond vector orientation and sampling 


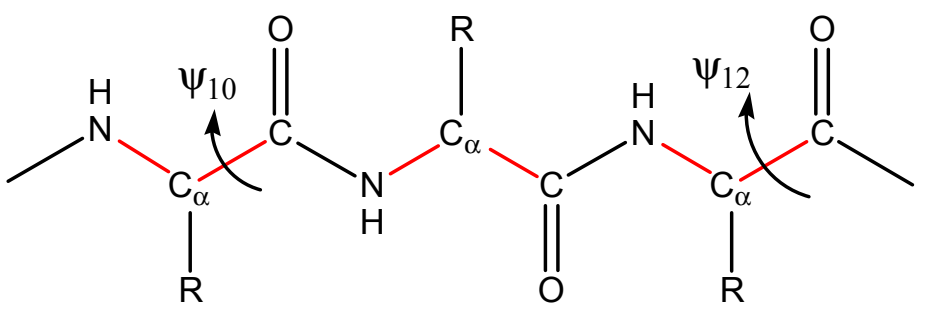
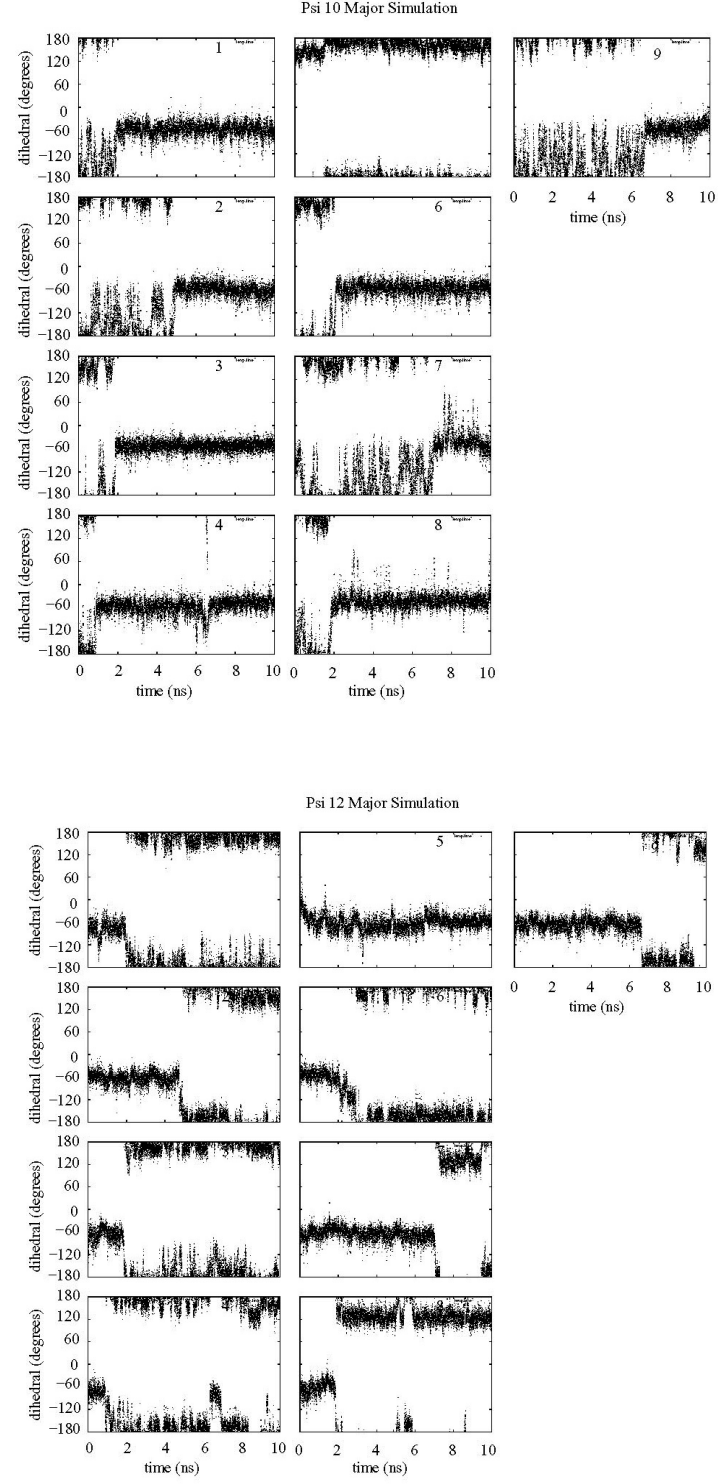

Figure 48: Dihedral $(\Psi)$ of residue 10 and 12 of the major conformation 


\subsection{Chloroperoxidase epoxidation}

I made contributions to an MD study of $\mathrm{CPO}$ aimed at elucidating the mechanism of the epoxidation reaction, which takes place at the active site. My contribution to the project was development of parameters for the sugar molecules and help with development of parameters for the substrate, cis- $\beta$-methylstyrene. In addition, I created patches to connect the heme to the proximal ligand, cysteine, and to connect sugar molecules to each other and to amino acids with covalent bonds. Project design and analysis of the results were carried out by other members of the research group, primarily Alexander Morozov. Below is the abstract from a paper published on this work [74]:

Molecular dynamics simulations of an explicitly solvated cis- $\beta$ methylstyrene /chloroperoxidase-Compound I complex are performed to determine the cause of the high enantiospecificity of epoxidation. From the simulations, a two-dimensional free energy potential is calculated to distinguish binding potential wells from which reaction to $1 \mathrm{~S} 2 \mathrm{R}$ and $1 \mathrm{R} 2 \mathrm{~S}$ epoxide products may occur. Convergence of the free energy potential is accelerated with an adaptive biasing potential. Analysis of binding is followed by analysis of $1 \mathrm{~S} 2 \mathrm{R}$ and $1 \mathrm{R} 2 \mathrm{~S}$ reaction precursor structures in which the substrate, having left the binding wells, places its reactive double bond in steric proximity to the oxyferryl heme center. Structural analysis of binding and reaction precursor conformations is presented. We find that i) a distortion of Glu183 is important for CPO-catalyzed epoxidation as was postulated previously based on experimental results; ii) the free energy of binding does not provide significant differentiation between structures leading to the respective epoxide enantiomers; iii) CPO's enantiospecificity toward cis- $\beta$-methylstyrene is likely to be caused by a specific group of residues which form a hydrophobic core surrounding the oxyferryl heme center. 


\section{REFERENCES}

1. Shaw, P.D. and L.P. Hager, An enzymic chlorination reaction. J. Am. Chem. Soc., 1959. 81: p. 1011-12.

2. Shaw, P.D. and L.P. Hager, Biological chlorination. VI. Chloroperoxidase: a component of the $\beta$-ketoadipate chlorinase system. J. Biol. Chem., 1961. 236: p. 1626-30.

3. Sundaramoorthy, M., J. Terner, and T.L. Poulos, The crystal structure of chloroperoxidase: a heme peroxidase-cytochrome P450 functional hybrid. Structure (London), 1995. 3: p. 1367-77.

4. Neidleman, S.L. and J. Geigert, Biological halogenation: roles in nature, potential in industry. Endeavour, 1987. 11: p. 5-15.

5. Wang, X., Special Topics in Biochemistry Lecture Notes, Lecture 8, Slide 13, 2010 .

6. Casella, L., et al., The Chloroperoxidase-Catalyzed Oxidation of Phenols. Mechanism, Selectivity, and Characterization of Enzyme-Substrate Complexes. Biochemistry, 1994. 33: p. 6377-86.

7. Thomas, J.A., D.R. Morris, and L.P. Hager, Chloroperoxidase. VIII. Formation of peroxide and halide complexes and their relation to the mechanism of the halogenation reaction. J. Biol. Chem., 1970. 245: p. 3135-42.

8. Bello-Ramirez, A.M., B.Y. Carreon-Garabito, and A.A. Nava-Ocampo, A theoretical approach to the mechanism of biological oxidation of organophosphorus pesticides. Toxicology, 2000. 149: p. 63-68.

9. Carlsen, L. and P. Lassen, Enzymically mediated formation of chlorinated humic acids. Org. Geochem., 1992. 18: p. 477-80.

10. Casey, W.H., Geochemistry: The fate of chlorine in soils. Science (Washington, DC, U. S.), 2002. 295: p. 985-986.

11. Niedan, V., I. Pavasars, and G. Oberg, Chloroperoxidase-mediated chlorination of aromatic groups in fulvic acid. Chemosphere, 2000. 41: p. 779-785.

12. Ortiz-Bermudez, P., E. Srebotnik, and K.E. Hammel, Chlorination and cleavage of lignin structures by fungal chloroperoxidases. Appl. Environ. Microbiol., 2003. 69: p. 5015-5018. 
13. Reina, R.G., A.C. Leri, and S.C.B. Myneni, Cl K-edge X-ray Spectroscopic Investigation of Enzymatic Formation of Organochlorines in Weathering Plant Material. Environ. Sci. Technol., 2004. 38: p. 783-789.

14. Walter, B. and K. Ballschmiter, Biohalogenation as a source of halogenated ansioles in air. Chemosphere, 1991. 22: p. 557-67.

15. Franssen, M.C.R. and d.P.H.C. Van, The chlorination of barbituric acid and some of its derivatives by chloroperoxidase. Bioorg. Chem., 1987. 15: p. 59-70.

16. Geigert, J., et al., Haloperoxidases: enzymic synthesis of $\alpha, \beta$-halohydrins from gaseous alkenes. Appl. Environ. Microbiol., 1983. 45: p. 366-74.

17. Griffin, B.W., Mechanism of halide-stimulated activity of chloroperoxidase. Evidence for enzymatic formation of free hypohalous acid. Biochem. Biophys. Res. Commun., 1983. 116(3): p. 873-9.

18. Griffin, B.W. and P.L. Ashley, Evidence for a radical mechanism of halogenation of monochlorodimedone catalyzed by chloroperoxidase. Arch. Biochem. Biophys., 1984. 233: p. 188-96.

19. Griffin, B.W. and R. Haddox, Chlorination of NADH: similarities of the hydrogen hypochloride-supported and chloroperoxidase-catalyzed reactions. Arch. Biochem. Biophys., 1985. 239: p. 305-9.

20. Lee, T.D., et al., Neighboring group migration in enzyme-mediated halohydrin formation. Biochem. Biophys. Res. Commun., 1983. 110: p. 880-3.

21. Manoj, K.M., Chlorinations catalyzed by chloroperoxidase occur via diffusible intermediate(s) and the reaction components play multiple roles in the overall process. Biochim. Biophys. Acta, Proteins Proteomics, 2006. 1764: p. 1325-1339.

22. Dunford, H.B., et al., On the mechanism of chlorination by chloroperoxidase. Arch. Biochem. Biophys., 1987. 252: p. 292-302.

23. Libby, R.D., T.M. Beachy, and A.K. Phipps, Quantitating direct chlorine transfer from enzyme to substrate in chloroperoxidase-catalyzed reactions. J. Biol. Chem., 1996. 271: p. 21820-21827.

24. Libby, R.D., et al., Chloroperoxidase halogenation reactions. Chemical versus enzymic halogenating intermediates. J. Biol. Chem., 1982. 257(9): p. 5030-7.

25. Murphy, C.D., New frontiers in biological halogenation. J. Appl. Microbiol., 2003. 94: p. 539-548.

26. Ramakrishnan, K., et al., Stereoselectivity of chloroperoxidase-dependent halogenation. Biochemistry, 1983. 22(13): p. 3271-7. 
27. Wagenknecht, H.-A., C. Claude, and W.-D. Woggon, New enzyme models of chloroperoxidase. Improved stability and catalytic efficiency of iron porphyrinates containing a thiolato ligand. Helv. Chim. Acta, 1998. 81: p. 15061520 .

28. Wagenknecht, H.-A. and W.-D. Woggon, Identification of intermediates in the catalytic cycle of chloroperoxidase. Chem. Biol., 1997. 4: p. 367-372.

29. Woggon, W.-D., Metalloporphyrines as Active Site Analogues - Lessons from Enzymes and Enzyme Models. Acc. Chem. Res., 2005. 38: p. 127-136.

30. Woggon, W.D., H.A. Wagenknecht, and C. Claude, Synthetic active site analogues of heme-thiolate proteins. Characterization and identification of intermediates of the catalytic cycles of cytochrome P450cam and chloroperoxidase. J. Inorg. Biochem., 2001. 83: p. 289-300.

31. Wannstedt, C., D. Rotella, and J.F. Siuda, Chloroperoxidase mediated halogenation of phenols. Bull. Environ. Contam. Toxicol., 1990. 44(2): p. 282-7.

32. Fu, H., et al., Chloroperoxidase-catalyzed asymmetric synthesis: enantioselective reactions of chiral hydroperoxides with sulfides and bromohydroxylation of glycals. J. Org. Chem., 1992. 57: p. 7265-70.

33. Levine, S.D., S.L. Neidleman, and M. Oberc, An enzymic route to $\alpha$-bromo steroidal ketones. Tetrahedron, 1968. 24: p. 2979-84.

34. Neidleman, S.L., et al., The enzymatic halogenation of steroids. Tetrahedron Lett, 1966. 44: p. 5337-42.

35. Liu, K.K.C. and C.H. Wong, Enzymic halohydration of glycals. J. Org. Chem., 1992. 57: p. 3748-50.

36. Beckwith, J.R. and L.P. Hager, Biological chlorination. VIII. Late intermediates in the biosynthesis of caldariomycin. J. Biol. Chem., 1963. 238: p. 3091-4.

37. Hager, L.P., et al., Chloroperoxidase. II. Utilization of halogen anions. J. Biol. Chem., 1966. 241: p. 1769-77.

38. Kuehnel, K., et al., Crystal structures of chloroperoxidase with its bound substrates and complexed with formate, acetate, and nitrate. J. Biol. Chem., 2006. 281: p. 23990-23998.

39. Karelson, M., U. Maran, and A.R. Katritzky, Theoretical study of the keto-enol tautomerism in aqueous solutions. Tetrahedron, 1996. 52(34): p. 11325-11328. 
40. Nagy, P.I., G. Alagona, and C. Ghio, Theoretical investigation of tautomeric equilibria for isonicotinic acid, 4-pyridone, and acetylacetone in vacuo and in solution. J. Chem. Theory Comput., 2007. 3(4): p. 1249-1266.

41. Belova, N.V., et al., Tautomeric and conformational properties of beta-diketones. J. Mol. Struct., 2010. 978(1-3): p. 282-293.

42. Lee, D., et al., A theoretical study on keto-enol tautomerization involving simple carbonyl derivatives. J. Comput. Chem., 1997. 18(1): p. 56-69.

43. Pei, K.M., Y.F. Ma, and X.M. Zheng, Theoretical study on the intramolecular proton transfer reactions of 3-methyl-5-hydroxyisoxazole and its water complexes. J. Mol. Struct.-Theochem, 2007. 820(1-3): p. 107-111.

44. Freitag, M.A., et al., On the keto-enol tautomerization of malonaldehyde: An effective fragment potential study. J. Phys. Chem. A, 2007. 111(9): p. 1659-1666.

45. Cucinotta, C.S., et al., Ab initio molecular dynamics study of the keto-enol tautomerism of acetone in solution. ChemPhysChem, 2006. 7(6): p. 1229-1234.

46. Cramer, C.J., Essentials of Computational Chemistry: Theories and Models. 2002: John Wiley \& Sons Ltd. 562 pp.

47. Hinchliffe, A., Molecular Modelling for Beginners. 2003: Wiley. 428 pp.

48. Andrews, D.H., The relation between the Raman spectra an the structure of organic molecules. Phys. Rev., 1930. 36: p. 544-54.

49. Kettering, C.F., L.W. Shutts, and D.H. Andrews, A representation of the dynamic properties of molecules by mechanical models. Phys. Rev., 1930. 36: p. 53143.

50. Snyder, R.G. and J.H. Schachtschneider, A valence force field for saturated hydrocarbons. Spectrochim. Acta, 1965. 21: p. 169-95.

51. Brooks, B.R., et al., CHARMM: The biomolecular simulation program. J. Comput. Chem., 2009. 30(10): p. 1545-1614.

52. BioModeling and bioInformatics, E.U.o.T. Potentials. 2007; Available from: http://www.pumma.nl/index.php/Theory/Potentials.

53. M. J. Frisch, G.W.T., H. B. Schlegel, G. E. Scuseria, M. A. Robb, J. R. Cheeseman, V. G. Zakrzewski, J. A. Montgomery Jr., R. E. Stratmann, J. C. Burant, S. Dapprich, J. M. Millam, A. D. Daniels, K. N. Kudin, M. C. Strain, O. Farkas, J. Tomasi, V. Barone, M. Cossi, R. Cammi, B. Mennucci, C. Pomelli, C. Adamo, S. Clifford, J. Ochterski, G. A. Petersson, P. Y. Ayala, Q. Cui, K. Morokuma, P. Salvador, J. J. Dannenberg, D. K. Malick, A. D. Rabuck, K. Raghavachari, J. B. Foresman, J. Cioslowski, J. V. Ortiz, A. G. Baboul, B. B. 
Stefanov, G. Liu, A. Liashenko, P. Piskorz, I. Komaromi, R. Gomperts, R. L. Martin, D. J. Fox, T. Keith, M. A. Al-Laham, C. Y. Peng, A. Nanayakkara, M. Challacombe, P. M. W. Gill, B. Johnson, W. Chen, M. W. Wong, J. L. Andres, C. Gonzalez, M. Head-Gordon, E. S. Replogle, and J. A. Pople, GAUSSIAN 98 1998, Gaussian, Inc.: Pittsburgh PA.

54. Wiberg, K.B., A scheme for strain energy minimization. Application to the cycloalkanes. J. Am. Chem. Soc., 1965. 87: p. 1070-8.

55. Fletcher, R. and C.M. Reeves, Function minimization by conjugate gradients. The Computer Journal, 1964. 7(2): p. 149-154.

56. M. J. Frisch, G.W.T., H. B. Schlegel, G. E. Scuseria, M. A. Robb, J. R. Cheeseman, G. Scalmani, V. Barone, B. Mennucci, G. A. Petersson, H. Nakatsuji, M. Caricato, X. Li, H. P. Hratchian, A. F. Izmaylov, J. Bloino, G. Zheng, J. L. Sonnenberg, M. Hada, M. Ehara, K. Toyota, R. Fukuda, J. Hasegawa, M. Ishida, T. Nakajima, Y. Honda, O. Kitao, H. Nakai, T. Vreven, J. A. Montgomery, Jr., J. E. Peralta, F. Ogliaro, M. Bearpark, J. J. Heyd, E. Brothers, K. N. Kudin, V. N. Staroverov, R. Kobayashi, J. Normand, K. Raghavachari, A. Rendell, J. C. Burant, S. S. Iyengar, J. Tomasi, M. Cossi, N. Rega, J. M. Millam, M. Klene, J. E. Knox, J. B. Cross, V. Bakken, C. Adamo, J. Jaramillo, R. Gomperts, R. E. Stratmann, O. Yazyev, A. J. Austin, R. Cammi, C. Pomelli, J. W. Ochterski, R. L. Martin, K. Morokuma, V. G. Zakrzewski, G. A. Voth, P. Salvador, J. J. Dannenberg, S. Dapprich, A. D. Daniels, Ö. Farkas, J. B. Foresman, J. V. Ortiz, J. Cioslowski, and D. J. Fox, GAUSSIAN 09, 2009, Gaussian, Inc.: Wallingford CT.

57. Y. Shao, L.F.-M., Y. Jung, J. Kussmann, C. Ochsenfeld, $\quad$ S. T. Brown, A. T. B. Gilbert, L. V. Slipchenko,S. V. Levchenko, D. P. O'Neill, R. A. Distasio Jr., R. C. Lochan, T. Wang, G. J. O. Beran, N. A. Besley, J. M., Herbert, C. Y. Lin, T. Van Voorhis, S. H. Chien, A. Sodt, R. P. Steele, V. A. Rassolov, P. E. Maslen, P. P. Korambath, R. D. Adamson, B. Austin, J. Baker, E. F. C. Byrd, H. Dachsel, R. J. Doerksen, A. Dreuw, B. D. Dunietz, A. D. Dutoi, T. R. Furlani, S. R. Gwaltney, A. Heyden, S. Hirata, C.-P. Hsu, G. Kedziora, R. Z. Khalliulin, P. Klunzinger, A. M. Lee, M. S. Lee, W. Liang, I. Lotan, N. Nair, B. Peters, E. I. Proynov, P. A. Pieniazek, Y. M. Rhee, J. Ritchie, E. Rosta, C. D. Sherrill, A. C. Simmonett, J. E. Subotnik, H. L. Woodcock III, W. Zhang, A. T. Bell, A. K. Chakraborty, D. M. Chipman, F. J. Keil, A. Warshel, W. J. Hehre, H. F. Schaefer III, J. Kong, A. I. Krylov, P. M. W. Gill, M. Head-Gordon, Advances in methods and algorithms in a modern quantum chemistry program package. Phys. Chem. Chem. Phys., 2006. 8: p. 3172 - 3191.

58. Fukui, K., Formulation of the reaction coordinate. J. Phys. Chem., 1970. 74(23): p. 4161-4163.

59. Gonzalez, C. and H.B. Schlegel, Reaction path following in mass-weighted internal coordinates. J. Phys. Chem., 1990. 94(14): p. 5523-5527. 
60. Sheppard, D., R. Terrell, and G. Henkelman, Optimization methods for finding minimum energy paths. J. Chem. Phys., 2008. 128: p. 134106/1-134106/10.

61. Maragakis, P., et al., Adaptive nudged elastic band approach for transition state calculation. J. Chem. Phys., 2002. 117: p. 4651-4658.

62. Liu, H., et al., Parallel iterative reaction path optimization in ab initio quantum mechanical/molecular mechanical modeling of enzyme reactions. J. Chem. Phys., 2004. 121: p. 697-706.

63. Lee, Y.-S., V.W. Pike, and M. Hodoscek, Identification of the Transition States in the Inversion of 1,4-Benzodiazepines with the Ab Initio Replica Path Method. J. Phys. Chem. A, 2008. 112: p. 1604-1611.

64. Henkelman, G., B.P. Uberuaga, and H. Jonsson, A climbing image nudged elastic band method for finding saddle points and minimum energy paths. J. Chem. Phys., 2000. 113: p. 9901-9904.

65. Henkelman, G. and H. Jonsson, Improved tangent estimate in the nudged elastic band method for finding minimum energy paths and saddle points. J. Chem. Phys., 2000. 113: p. 9978-9985.

66. Henkelman, G., G. Johannesson, and H. Jonsson, Methods for finding saddle points and minimum energy paths. Prog. Theor. Chem. Phys., 2000. 5: p. 269302.

67. Elber, R. and M. Karplus, A method for determining reaction paths in large molecules: application to myoglobin. Chem. Phys. Lett., 1987. 139: p. 375-80.

68. Czerminski, R. and R. Elber, Reaction path study of conformational transitions in flexible systems: applications to peptides. J. Chem. Phys., 1990. 92: p. 5580-601.

69. Czerminski, R. and R. Elber, Self-avoiding walk between two fixed points as a tool to calculate reaction paths in large molecular systems. Int. J. Quantum Chem., Quantum Chem. Symp., 1990. 24: p. 167-86.

70. Cisneros, G.A., et al., Reaction path determination for quantum mechanical/molecular mechanical modeling of enzyme reactions by combining first order and second order "chain-of-replicas" methods. J. Chem. Phys., 2005. 122: p. 114502/1-114502/7.

71. Ayala, P.Y. and H.B. Schlegel, A combined method for determining reaction paths, minima, and transition state geometries. J. Chem. Phys., 1997. 107: p. 375384. 
72. Vanommeslaeghe, K., et al., CHARMM general force field: A force field for druglike molecules compatible with the CHARMM all-atom additive biological force fields. J. Comput. Chem., 2010. 31: p. 671-690.

73. Singh, U.C. and P.A. Kollman, An approach to computing electrostatic charges for molecules. J. Comput. Chem., 1984. 5: p. 129-45.

74. Morozov, A.N., et al., Enantiospecificity of Chloroperoxidase-Catalyzed Epoxidation: Biased Molecular Dynamics Study of a Cis- $\beta$ Methylstyrene/Chloroperoxidase-Compound I Complex. Biophys. J., 2011. 100: p. 1066-1075. 


\section{APPENDICES}

\section{Appendix A: Computer scripts}

A1 Scripts used in the study of limited-hydration CPD keto - enol tautomerization

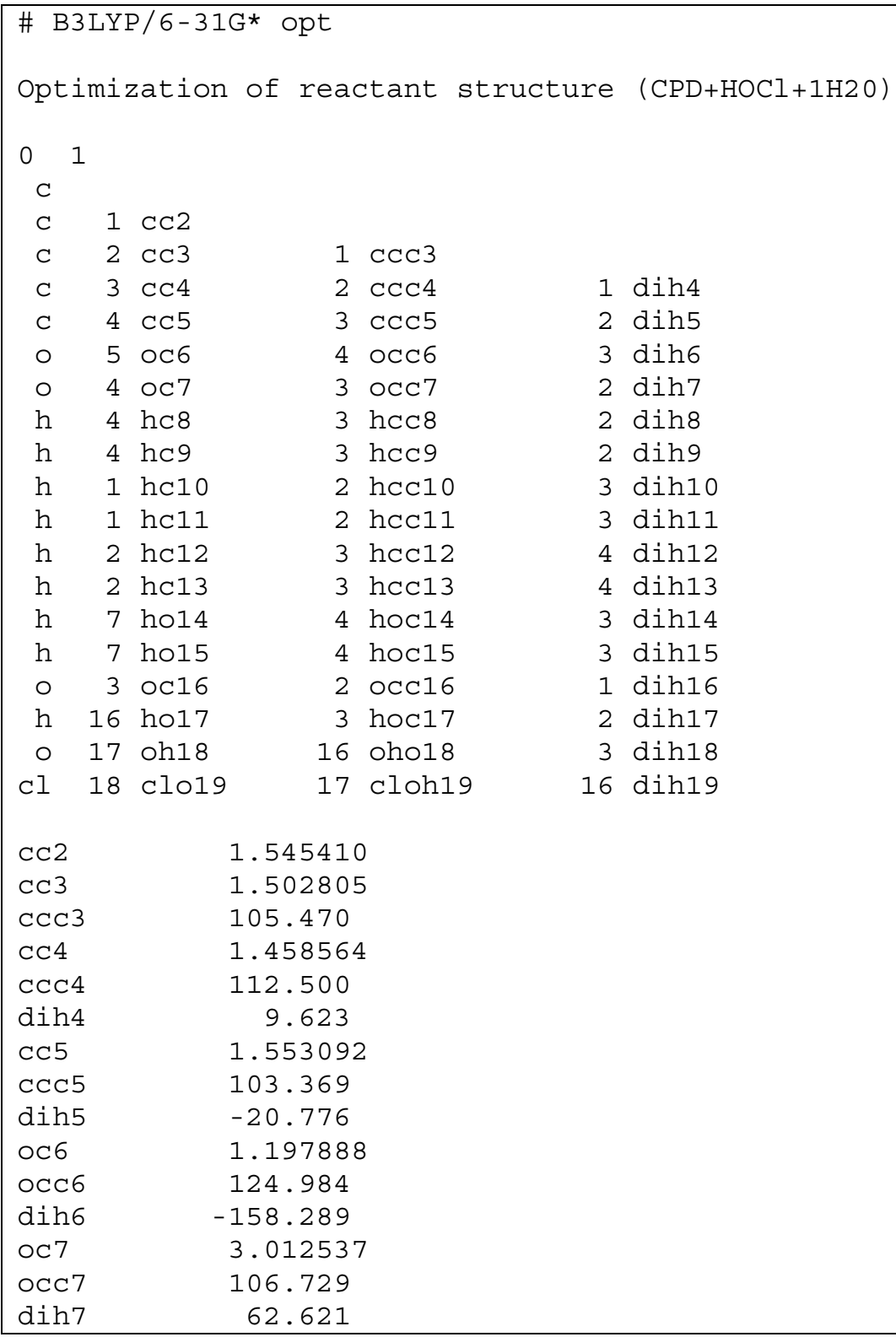




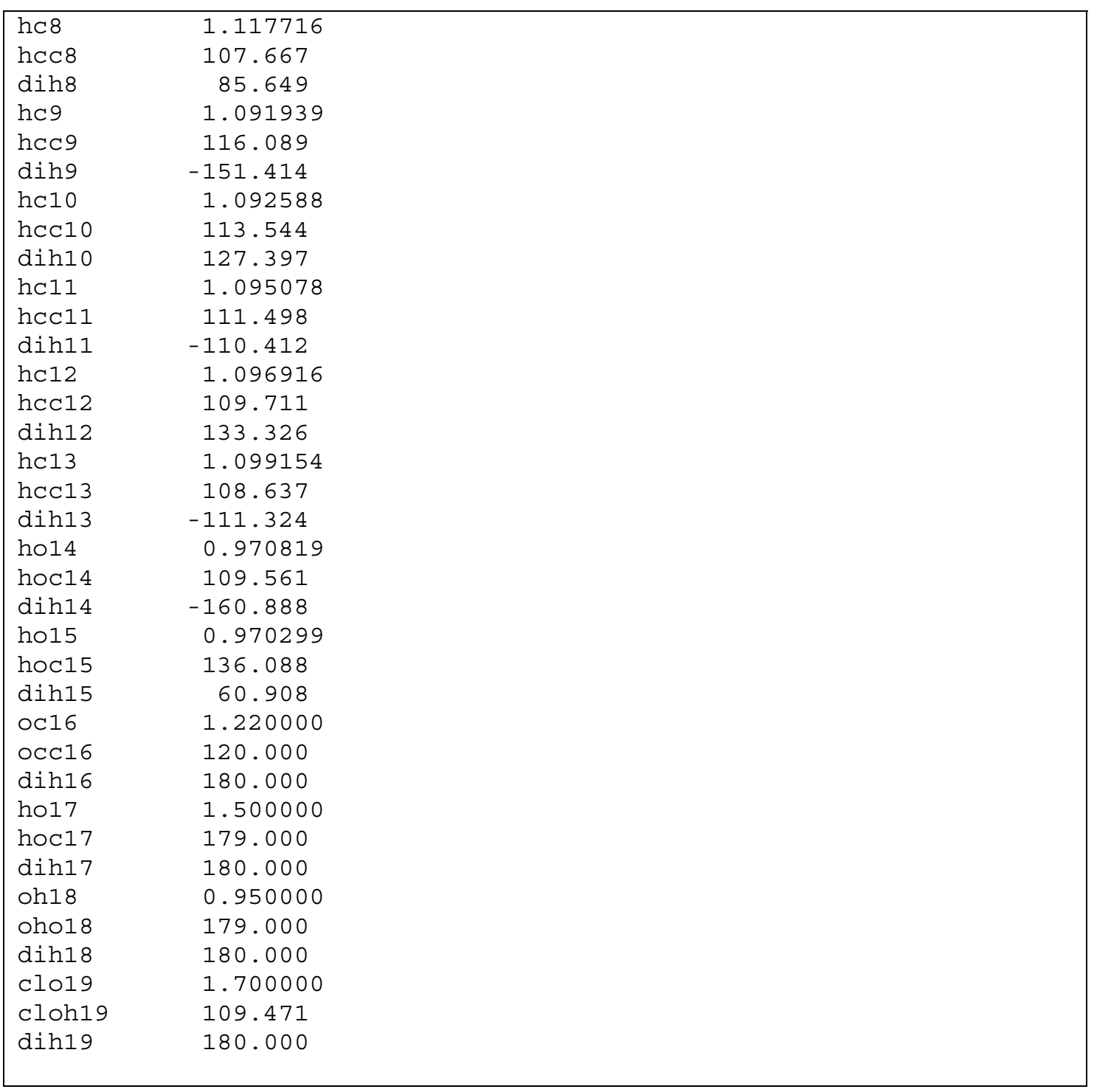

Table 26: Optimization script for reactant (or product) structure of CPD + HOCI + explicit water molecule

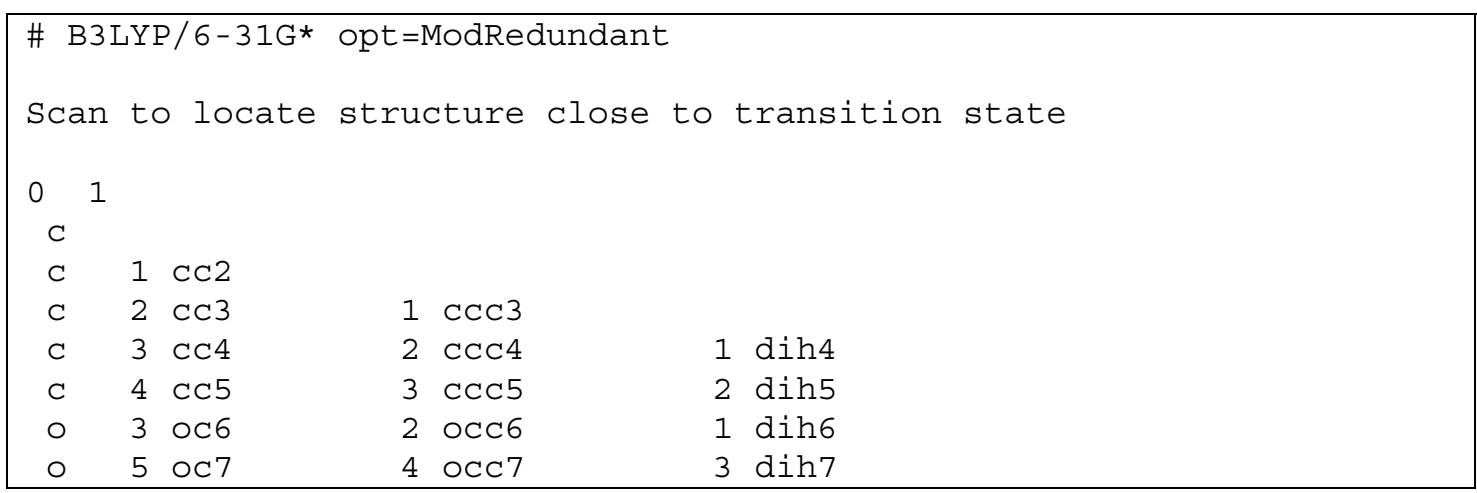




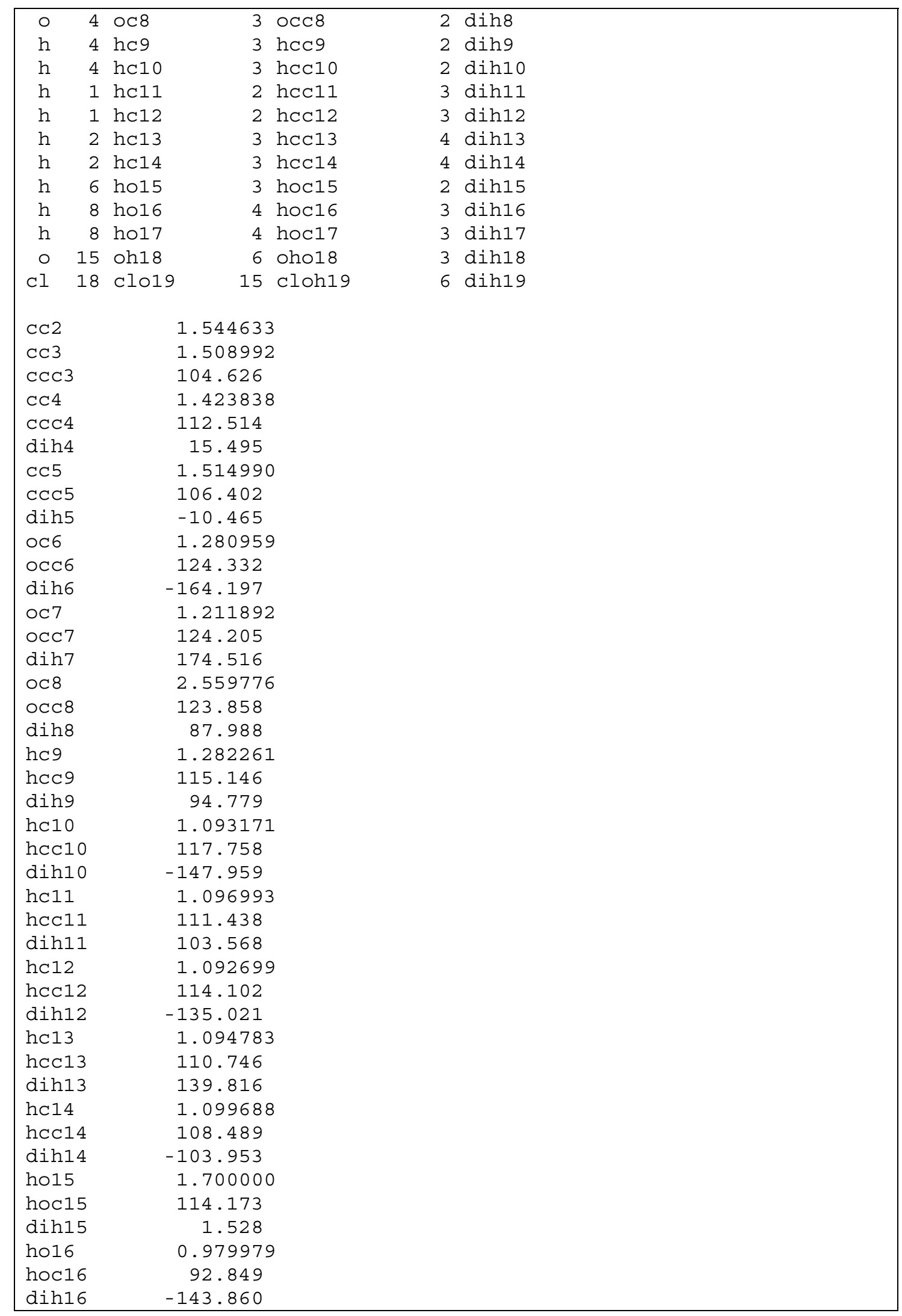




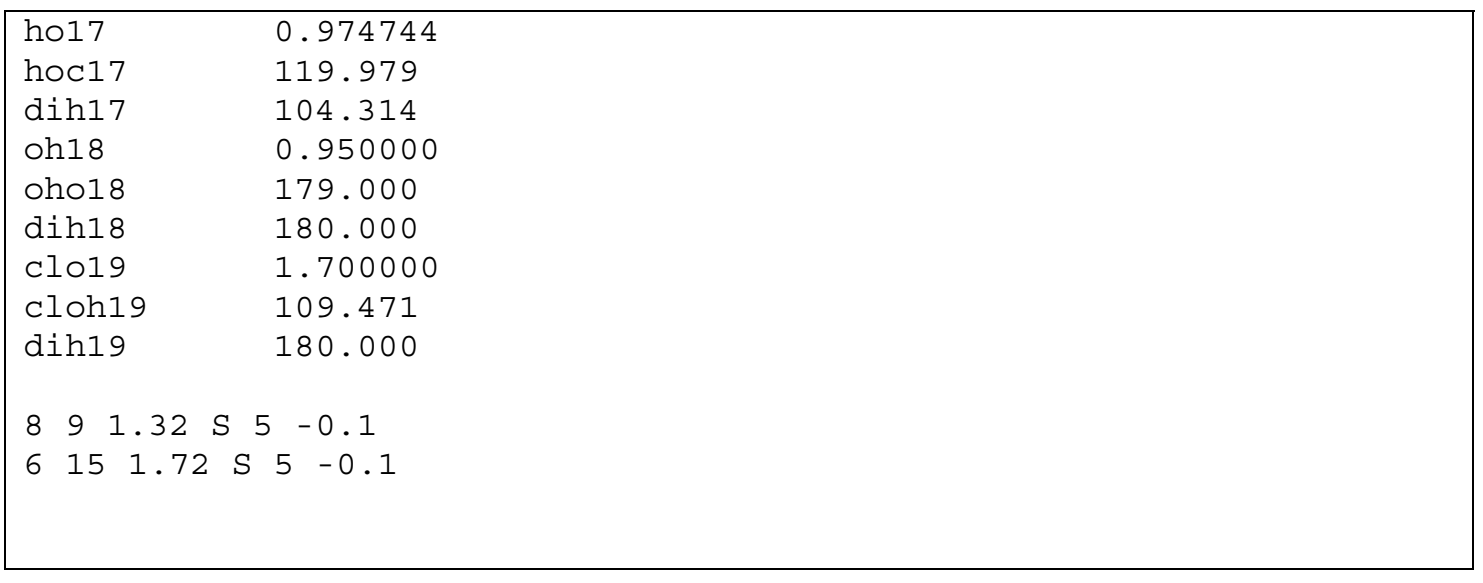

Table 27: Script for scan of potential energy surface to locate structure close to the transition state

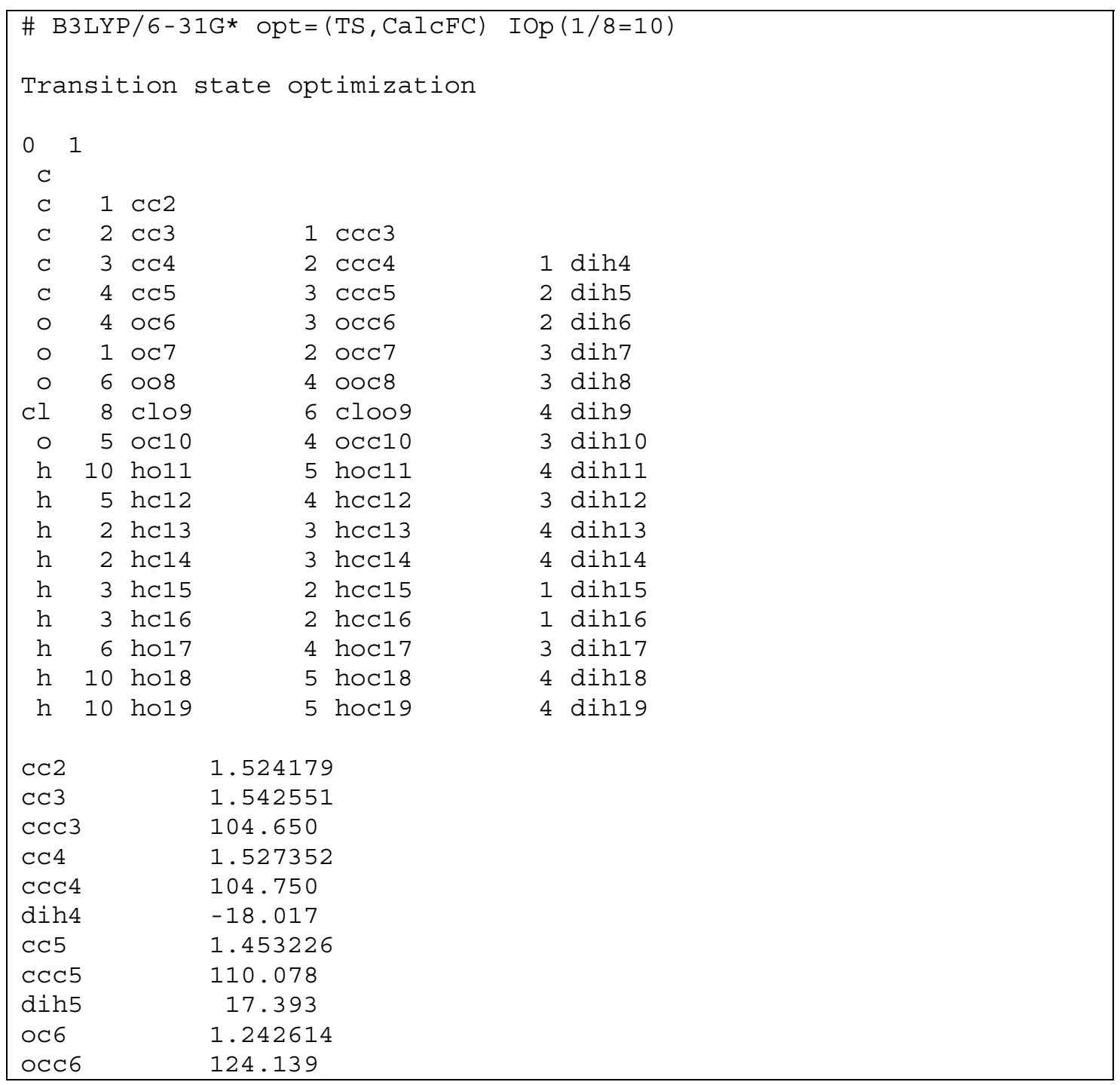




\begin{tabular}{|c|c|}
\hline dih6 & -162.496 \\
\hline OC7 & 1.233933 \\
\hline OCC 7 & 125.202 \\
\hline $\operatorname{dih} 7$ & -158.458 \\
\hline 008 & 2.330560 \\
\hline $00 c 8$ & 110.574 \\
\hline dih8 & 3.363 \\
\hline Clo9 & 1.725564 \\
\hline Cloog & 104.618 \\
\hline dih9 & 103.668 \\
\hline oc10 & 2.501081 \\
\hline occlo & 131.864 \\
\hline dih10 & 89.754 \\
\hline hol1 & 1.220000 \\
\hline hoc 11 & 14.063 \\
\hline dih11 & 30.876 \\
\hline hol2 & 1.093236 \\
\hline $\mathrm{hcc} 12$ & 116.890 \\
\hline $\operatorname{dih} 12$ & -145.272 \\
\hline hc13 & 1.098553 \\
\hline hecl3 & 110.979 \\
\hline $\operatorname{dih} 13$ & 98.138 \\
\hline hc14 & 1.093351 \\
\hline $\mathrm{hCCl} 4$ & 115.038 \\
\hline dih14 & -139.992 \\
\hline hc15 & 1.091907 \\
\hline hccl 5 & 115.884 \\
\hline dih15 & -139.659 \\
\hline hc16 & 1.098098 \\
\hline hccl 6 & 111.551 \\
\hline dih16 & 98.877 \\
\hline ho17 & 1.220000 \\
\hline hoc 17 & 113.730 \\
\hline $\operatorname{dih} 17$ & 3.751 \\
\hline ho18 & 1.002126 \\
\hline hoc18 & 80.741 \\
\hline dih18 & -136.840 \\
\hline ho19 & 0.973841 \\
\hline hoc19 & 112.608 \\
\hline $\operatorname{dih} 19$ & 117.714 \\
\hline
\end{tabular}

Table 28: Transition state optimization script

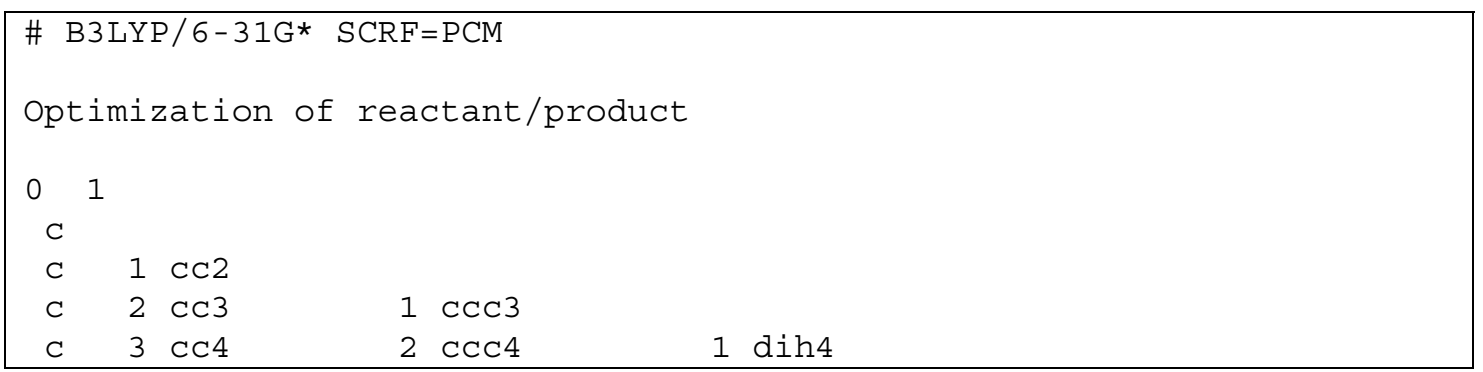




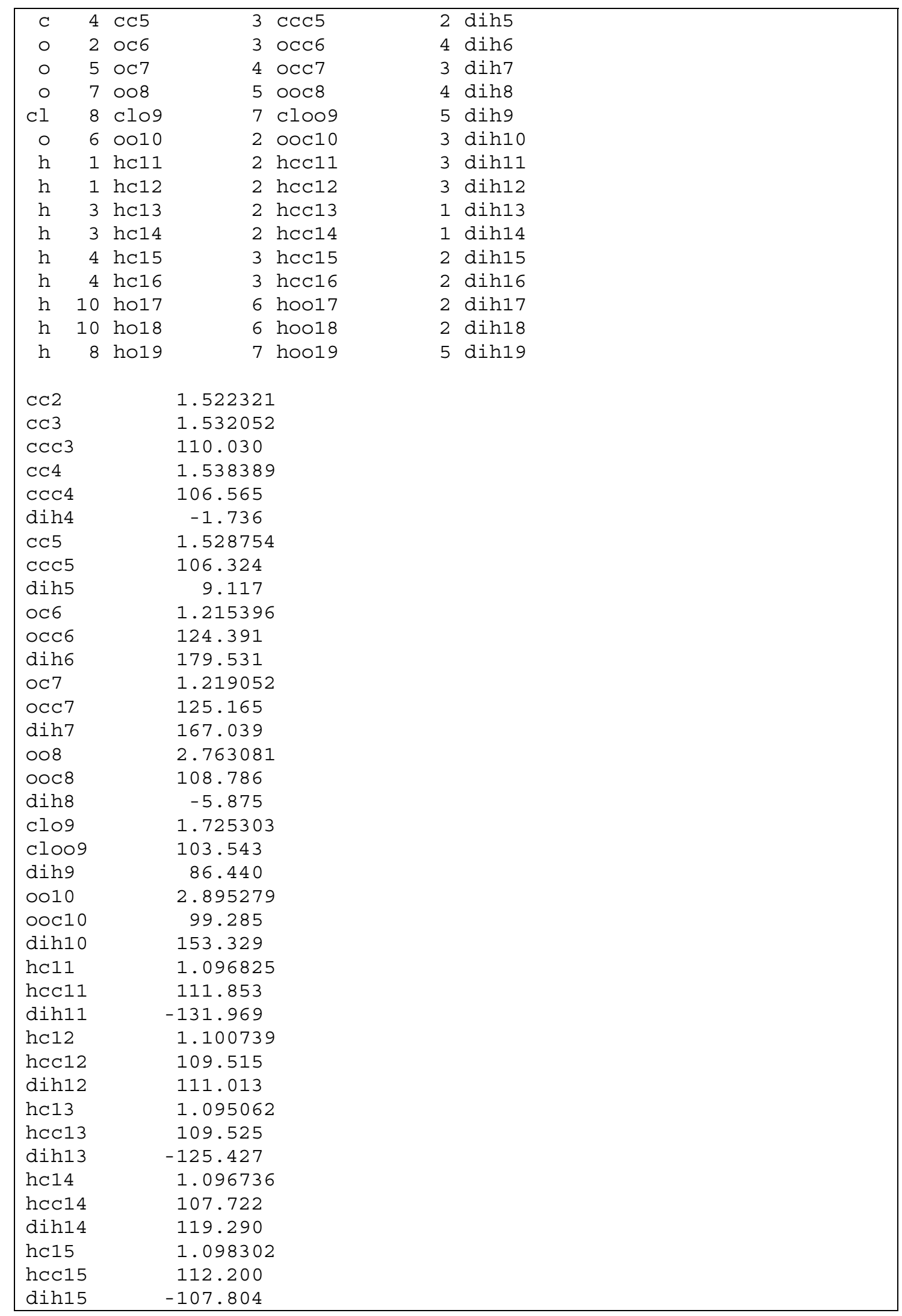




\begin{tabular}{|lc}
\hline hc16 & 1.093686 \\
hcc16 & 115.025 \\
dih16 & 131.078 \\
ho17 & 0.975757 \\
hoo17 & 18.144 \\
dih17 & 173.629 \\
ho18 & 0.968553 \\
hoo18 & 114.146 \\
dih18 & 113.727 \\
ho19 & 0.991861 \\
hoo19 & 9.666 \\
dih19 & 170.874
\end{tabular}

Table 29: Single point energy calculation script using implicit solvent environment (PCM), of gasphase optimized structure of reactant (or product) of CPD + HOCI + explicit water molecule 


\section{A2 Scripts used in the development of parameters for CPD (and $\mathrm{HOCl}$ )}

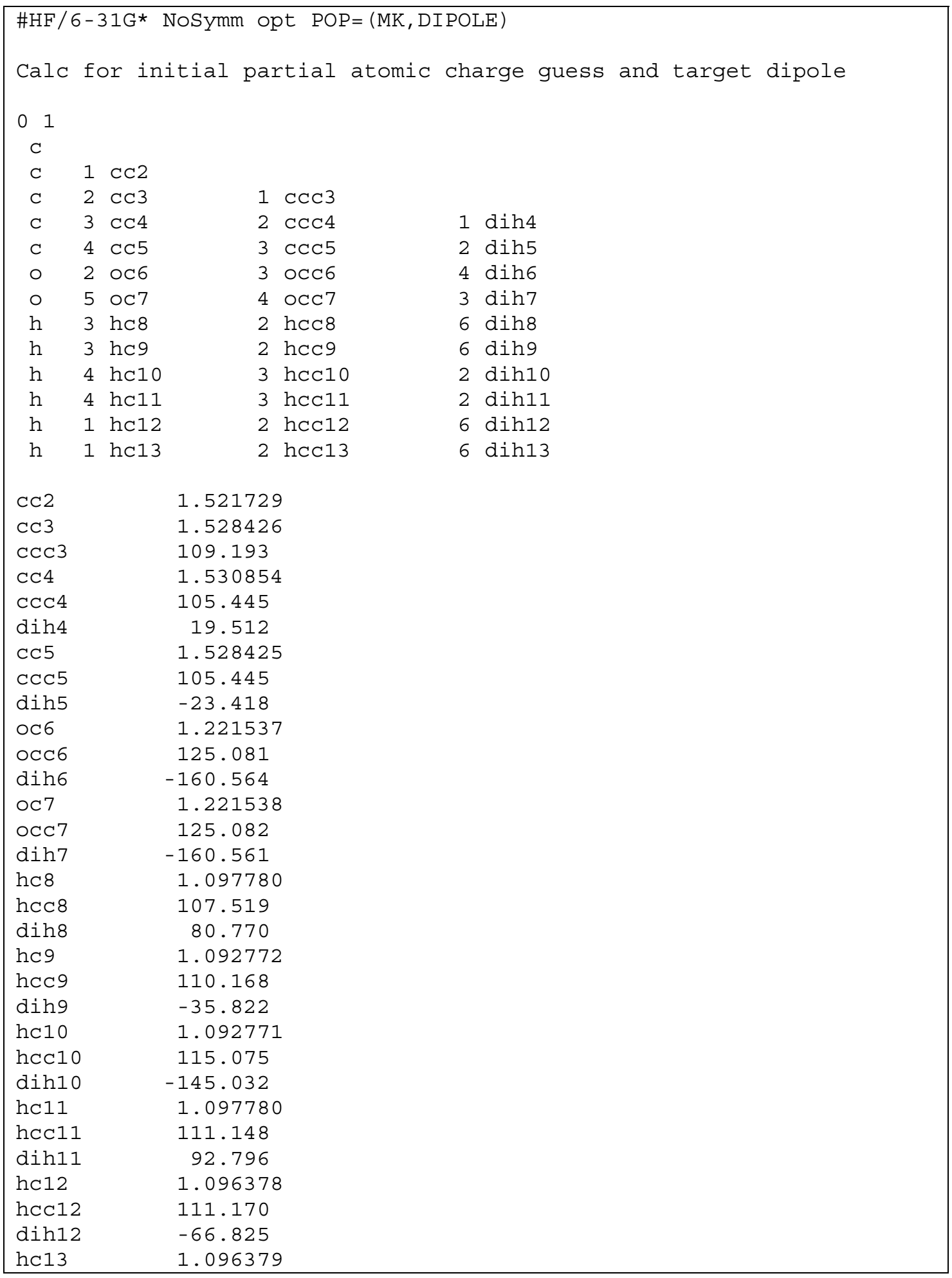




$\begin{array}{rr}\text { hec13 } & 111.260 \\ \operatorname{dih} 13 & 51.984\end{array}$

Table 30: Parameterization script (QM): Initial partial atomic guess and target dipole calculation

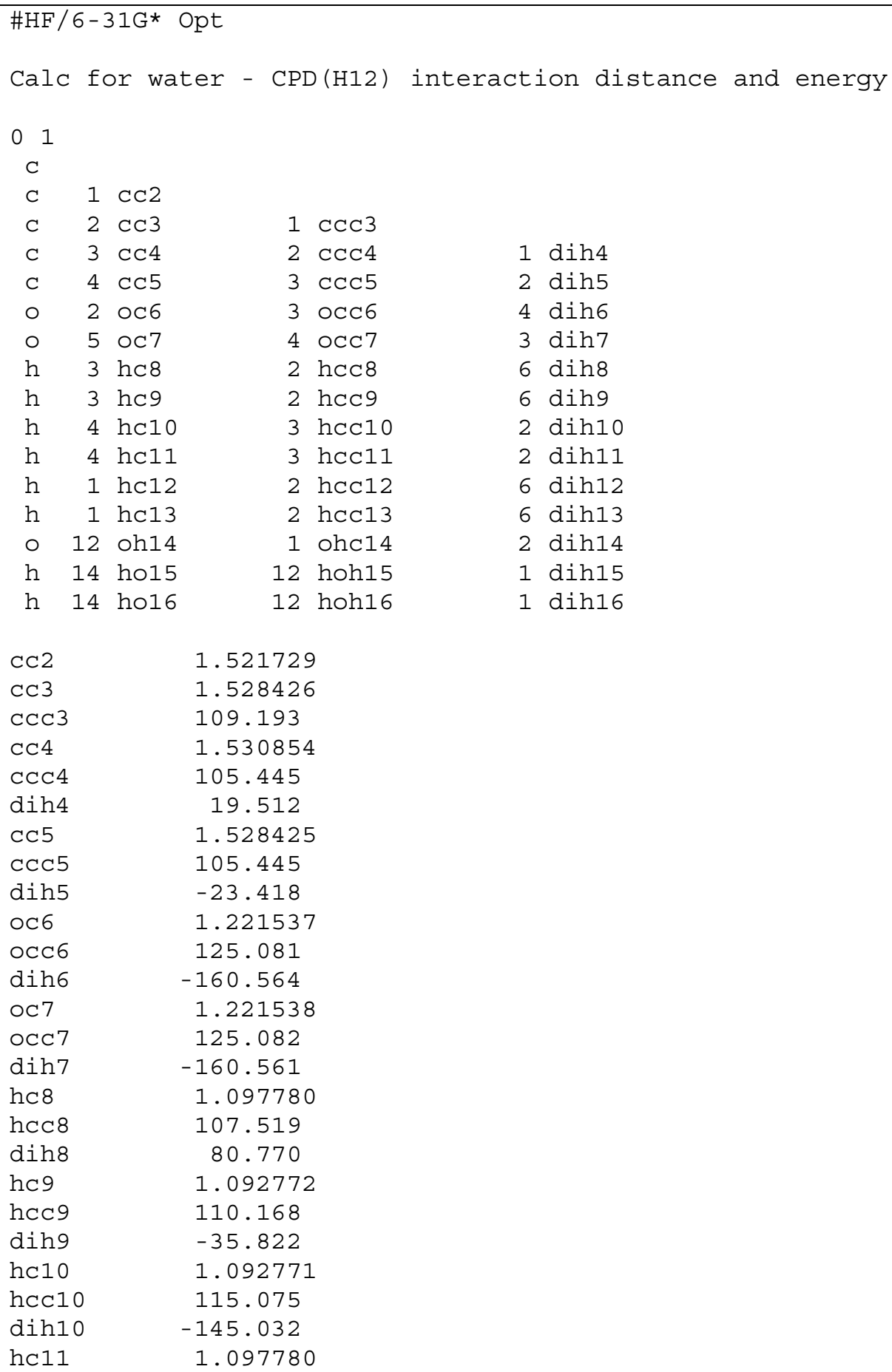




\begin{tabular}{|lc}
\hline hcc11 & 111.148 \\
dih11 & 92.796 \\
hc12 & 1.096378 \\
hcc12 & 111.170 \\
dih12 & -66.825 \\
hc13 & 1.096379 \\
hcc13 & 111.260 \\
dih13 & 51.984 \\
oh14 & 2.100000 \\
ohc14 & 179.000 \\
dih14 & 180.000 \\
ho15 & 0.950000 \\
hoh15 & 109.471 \\
dih15 & 180.000 \\
hol6 & 0.950000 \\
hoh16 & 109.471 \\
dih16 & 300.000 \\
\end{tabular}

Table 31: Parameterization script (QM): Target water-CPD (H12) interaction energy and distance calculation

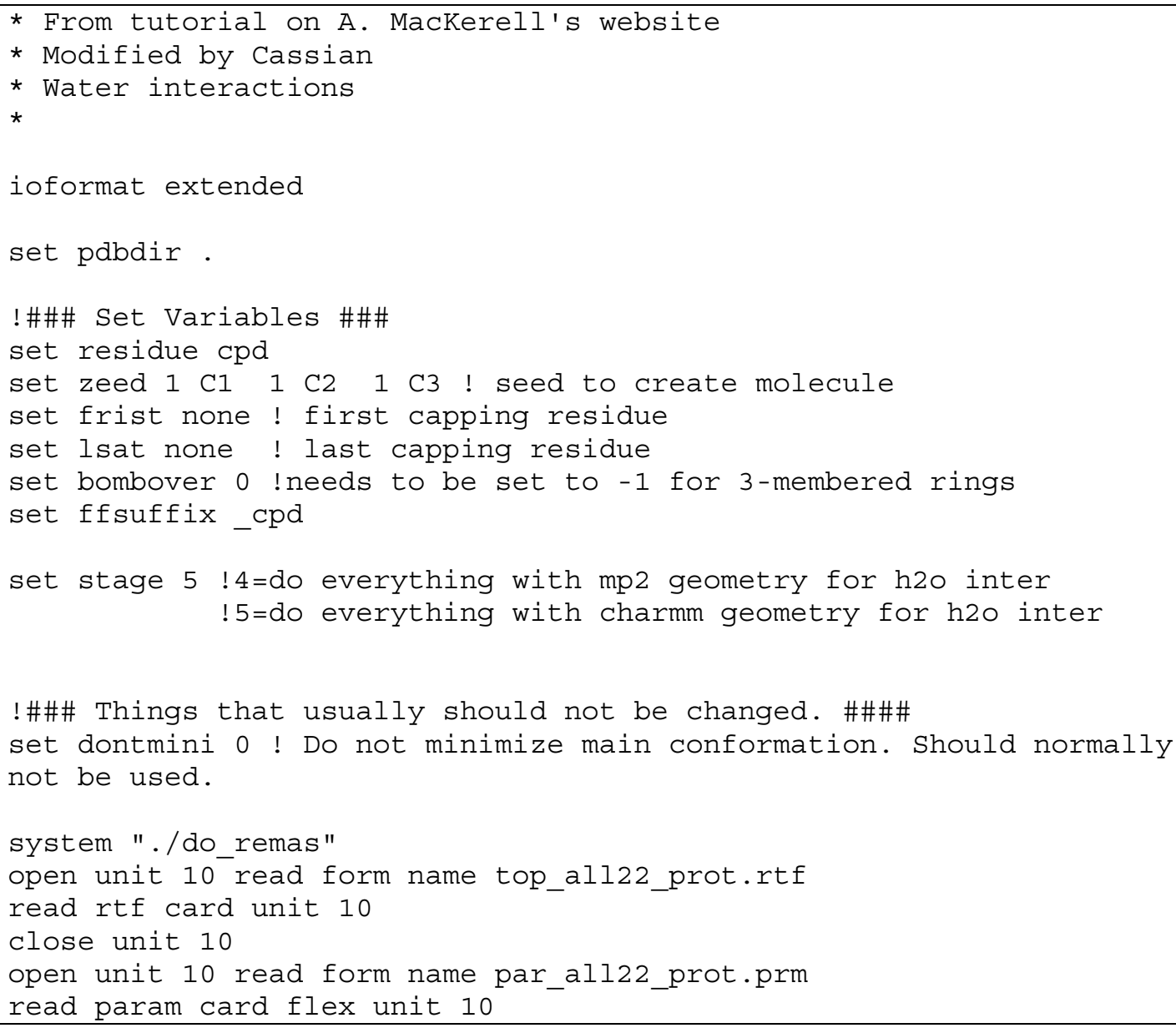




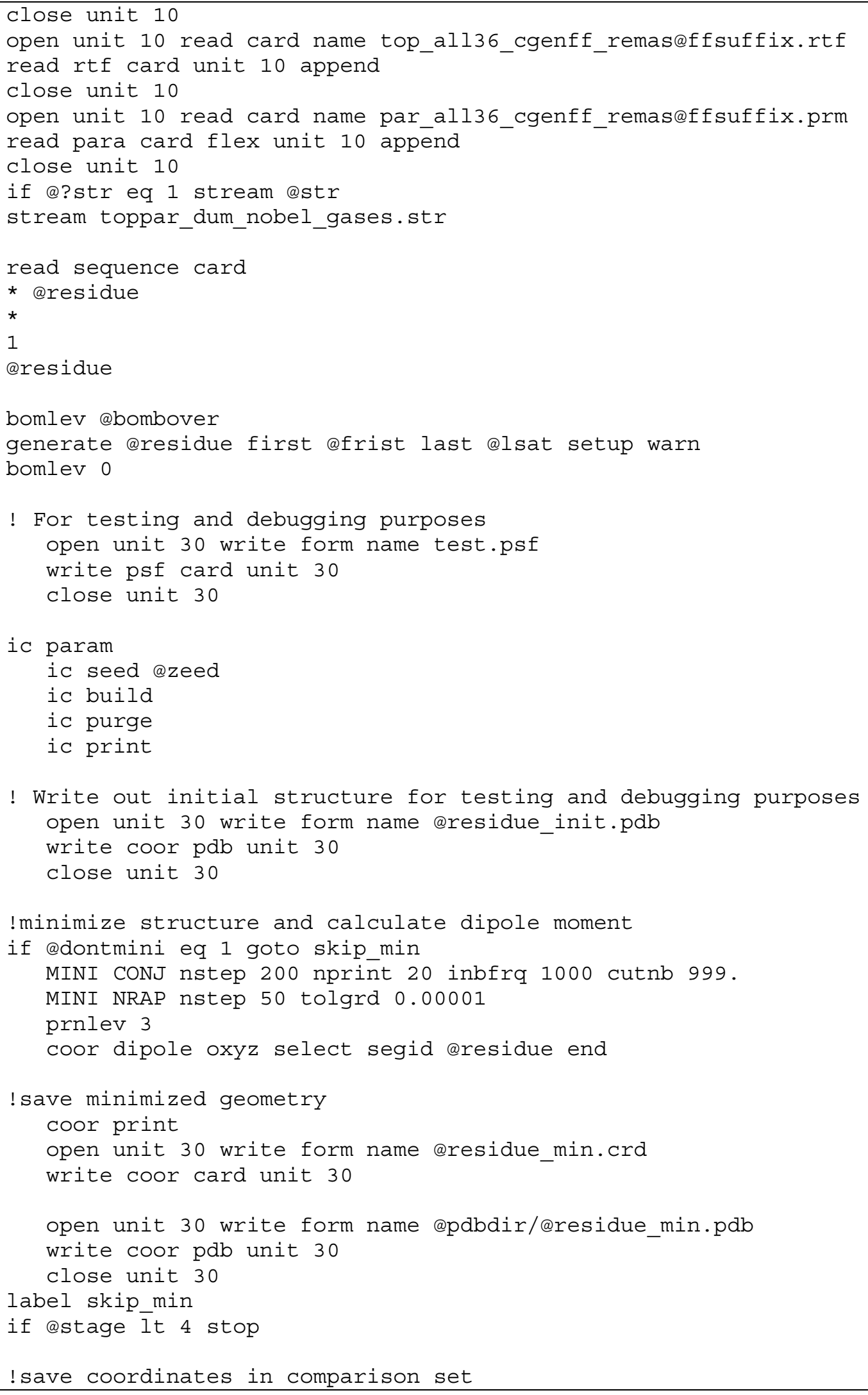




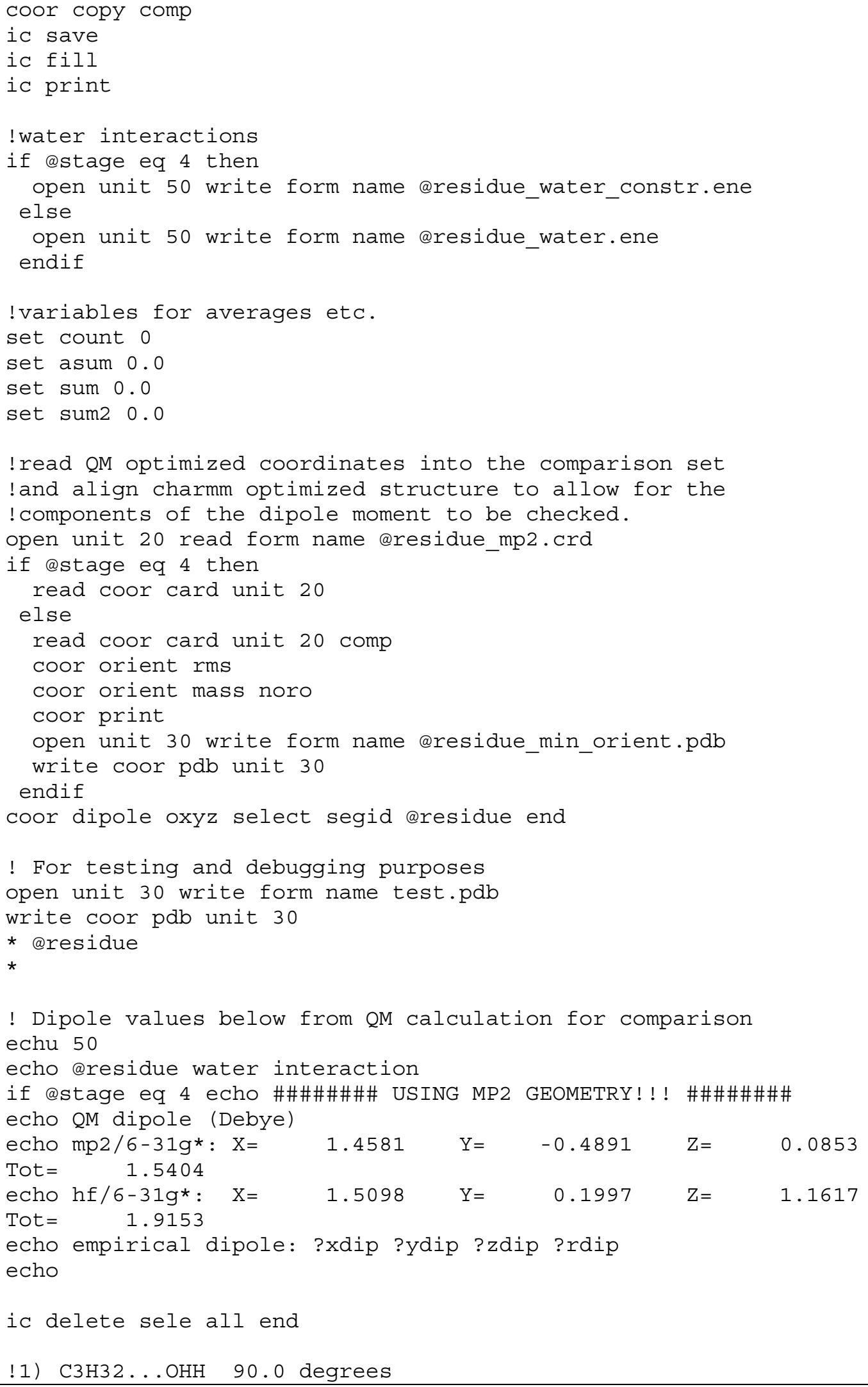




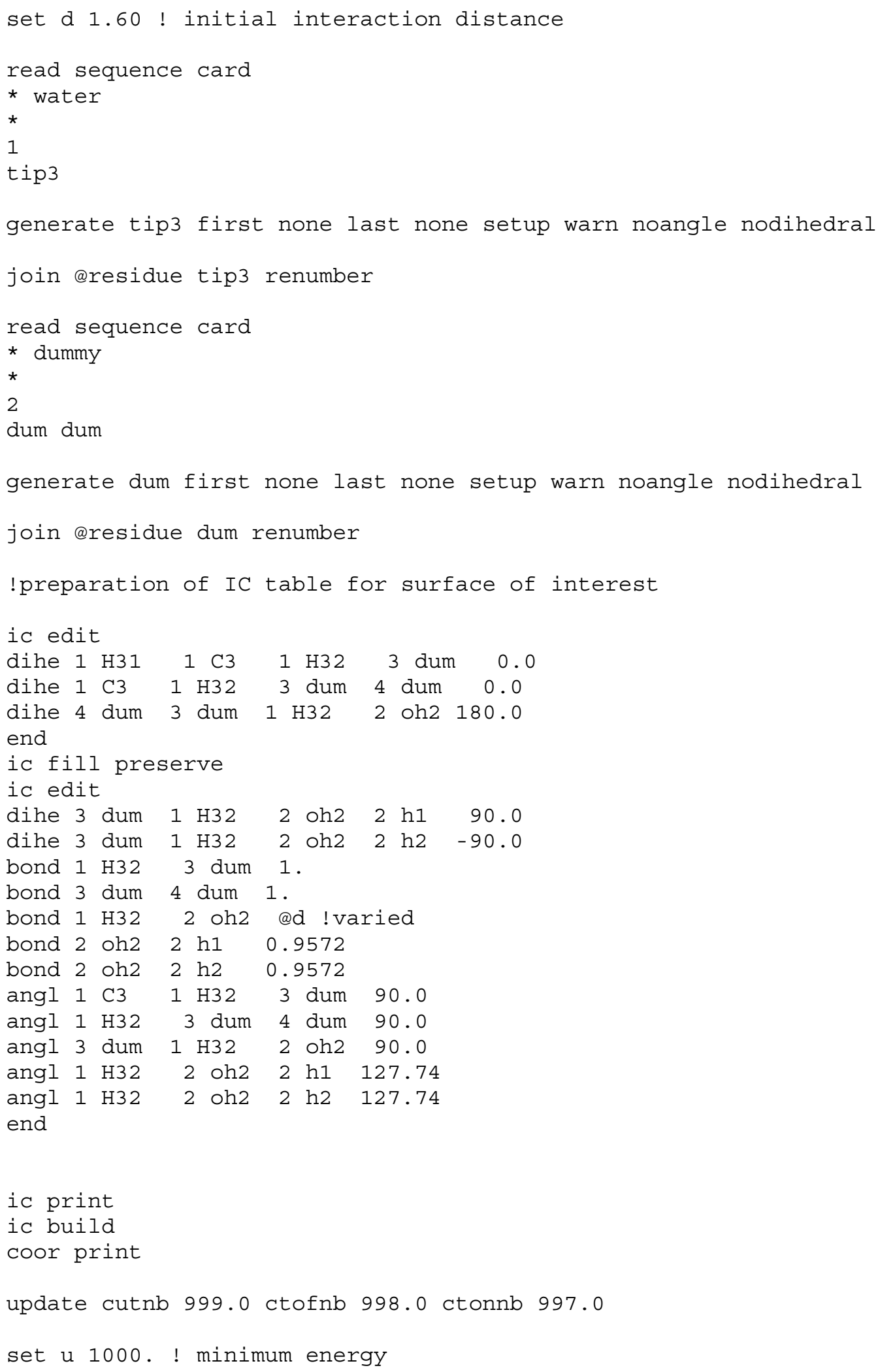




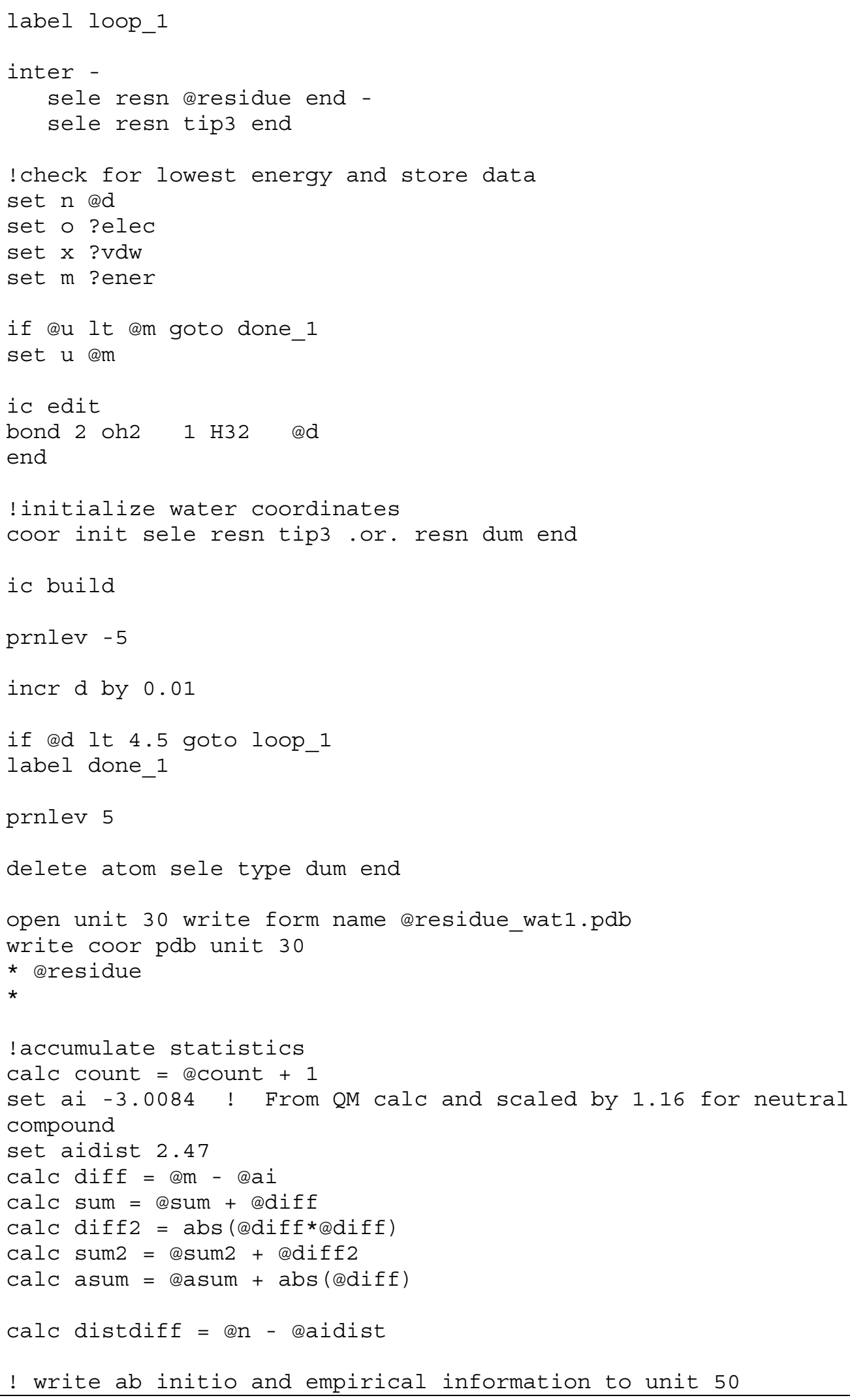




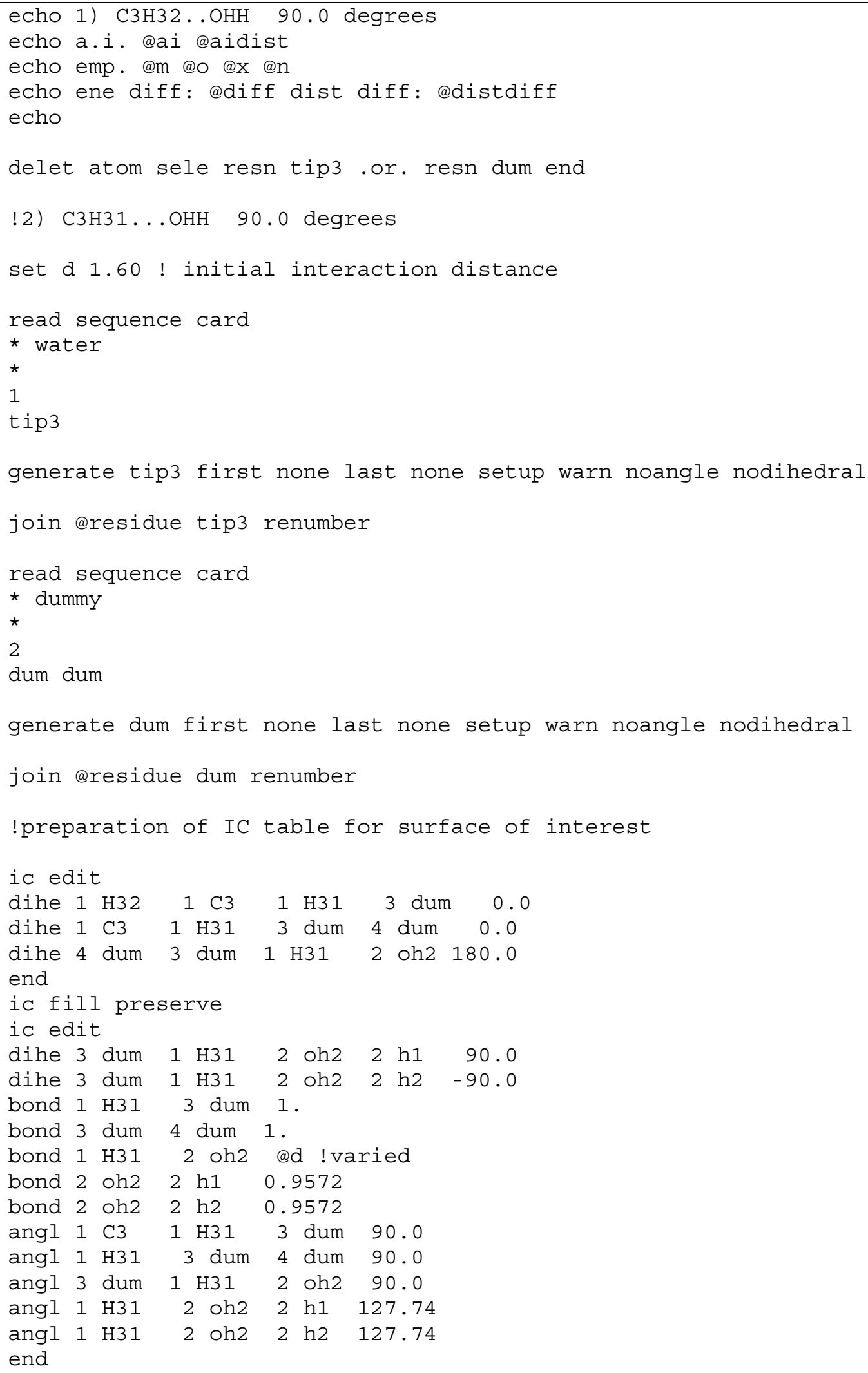




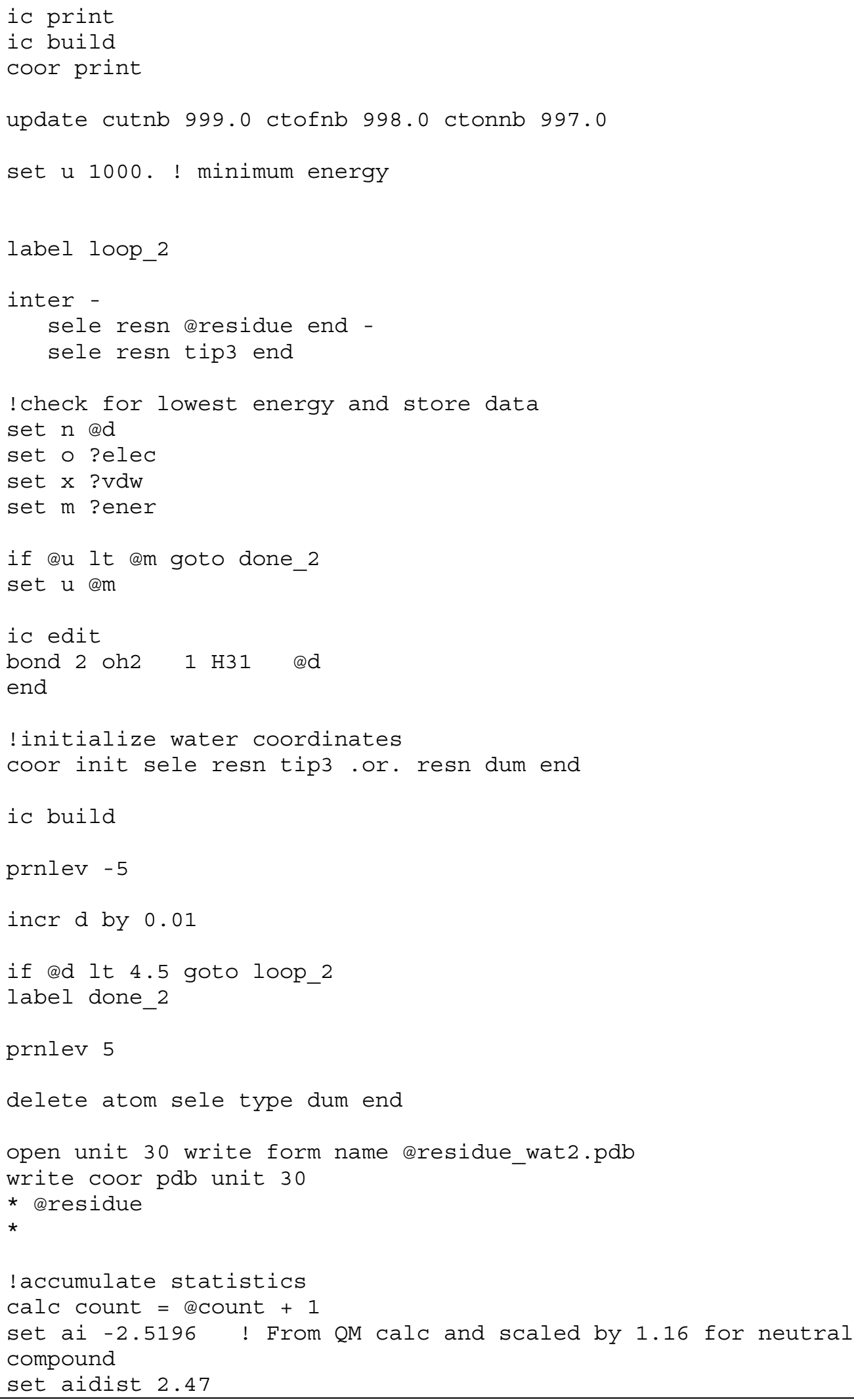




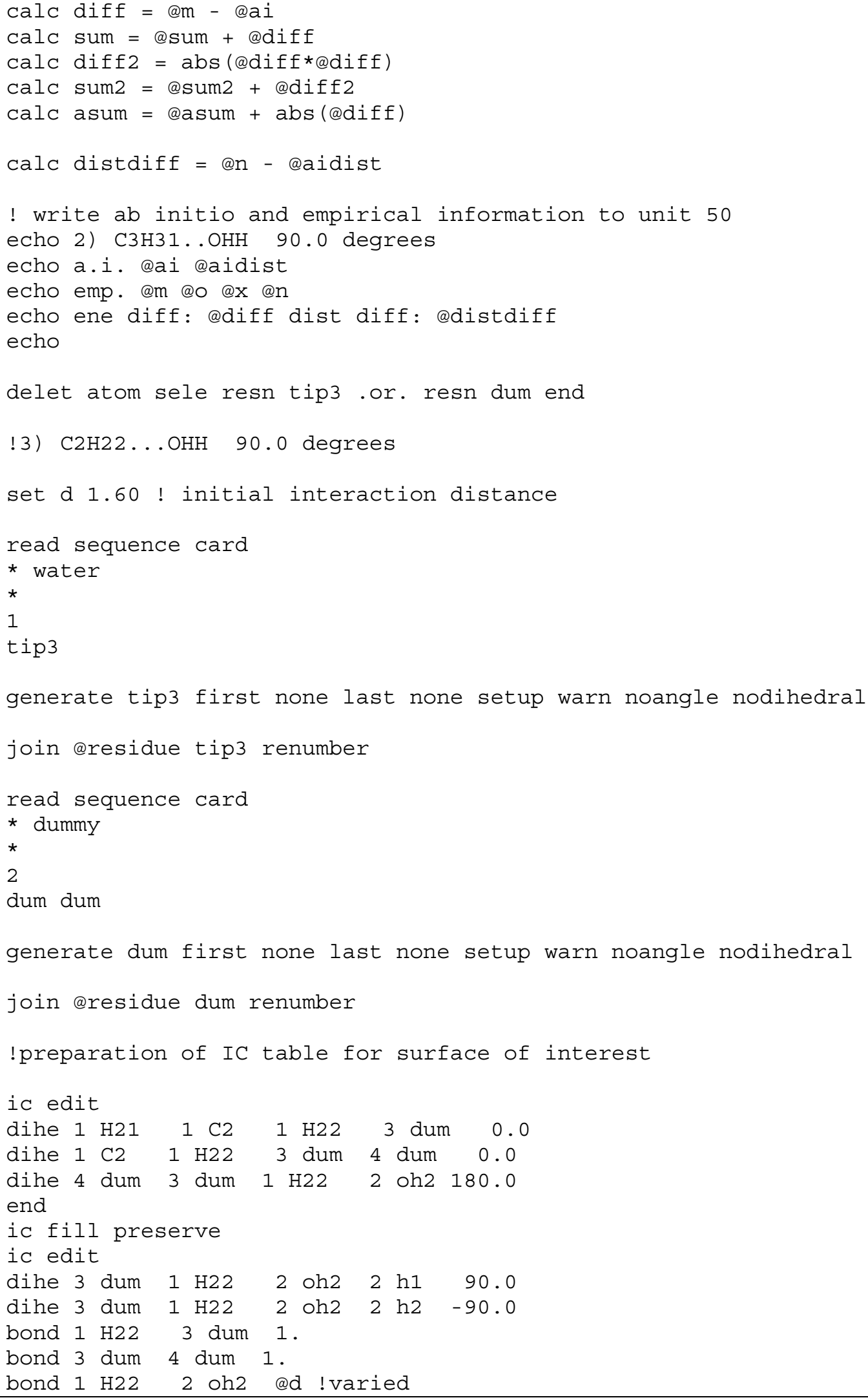




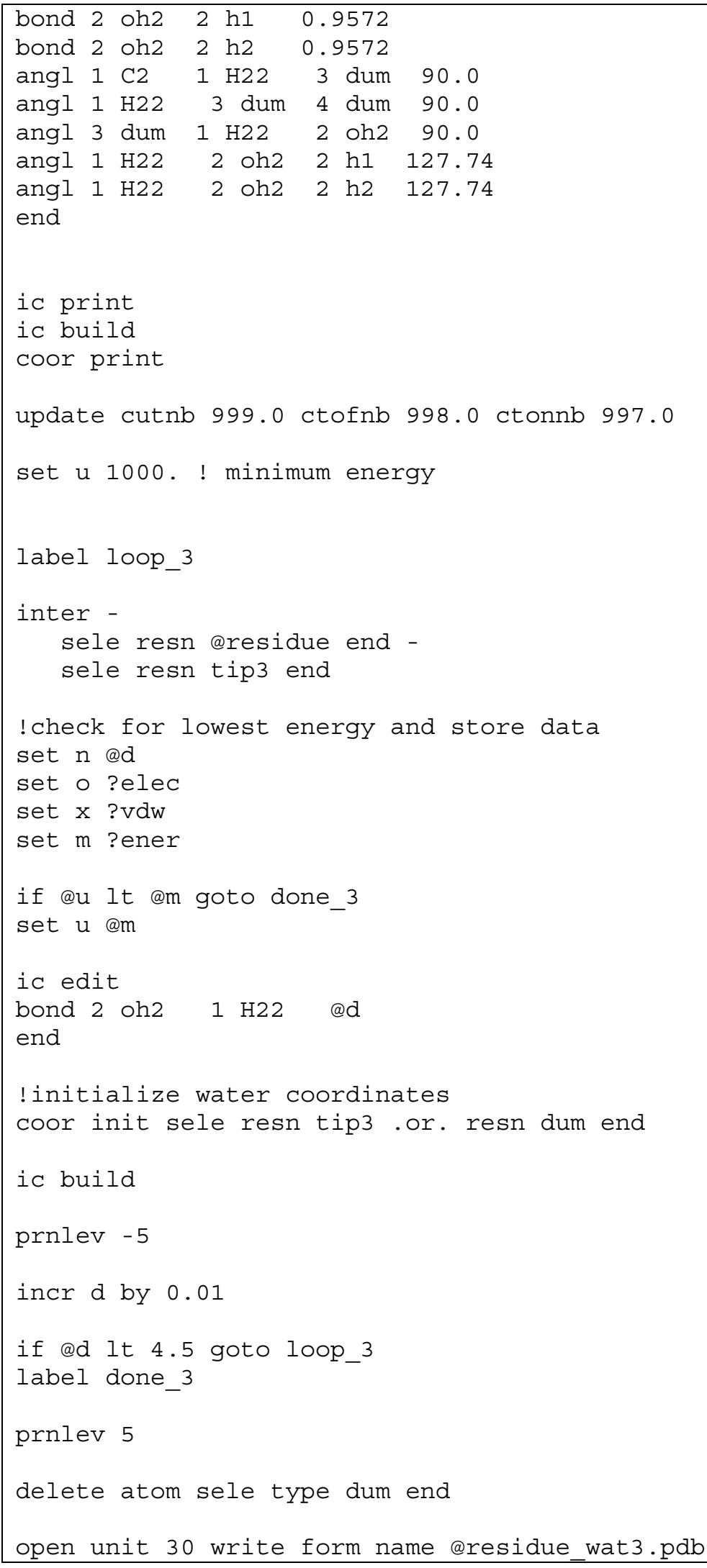




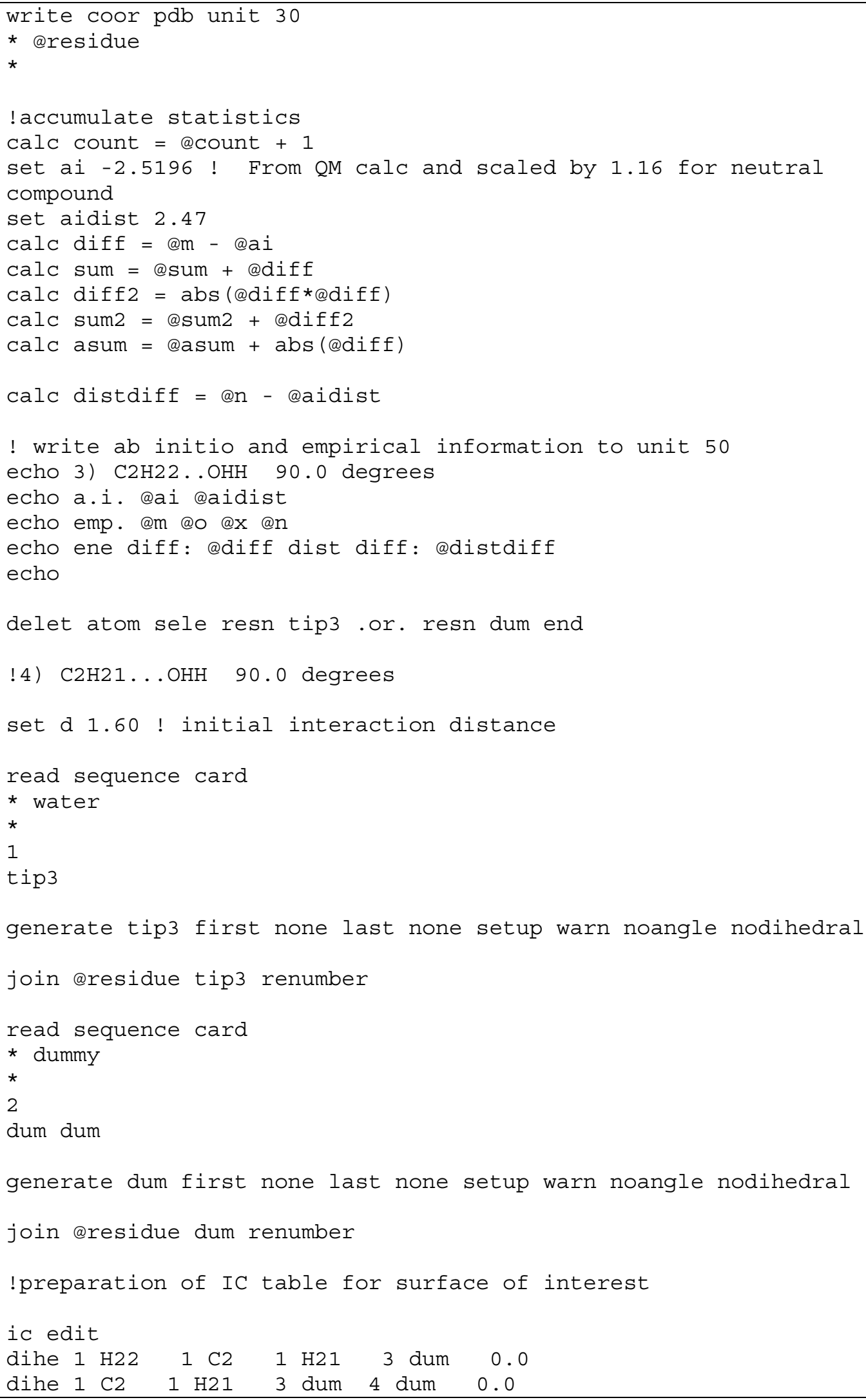




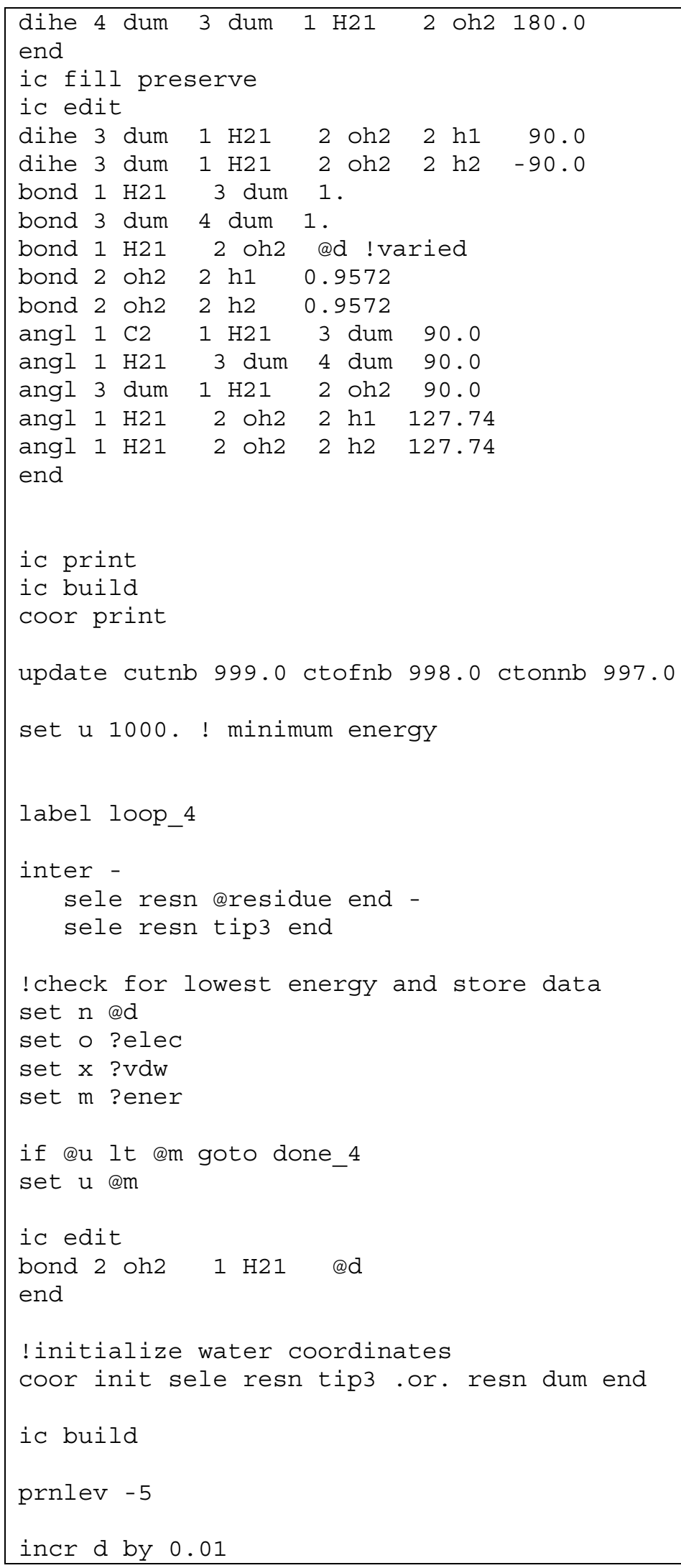




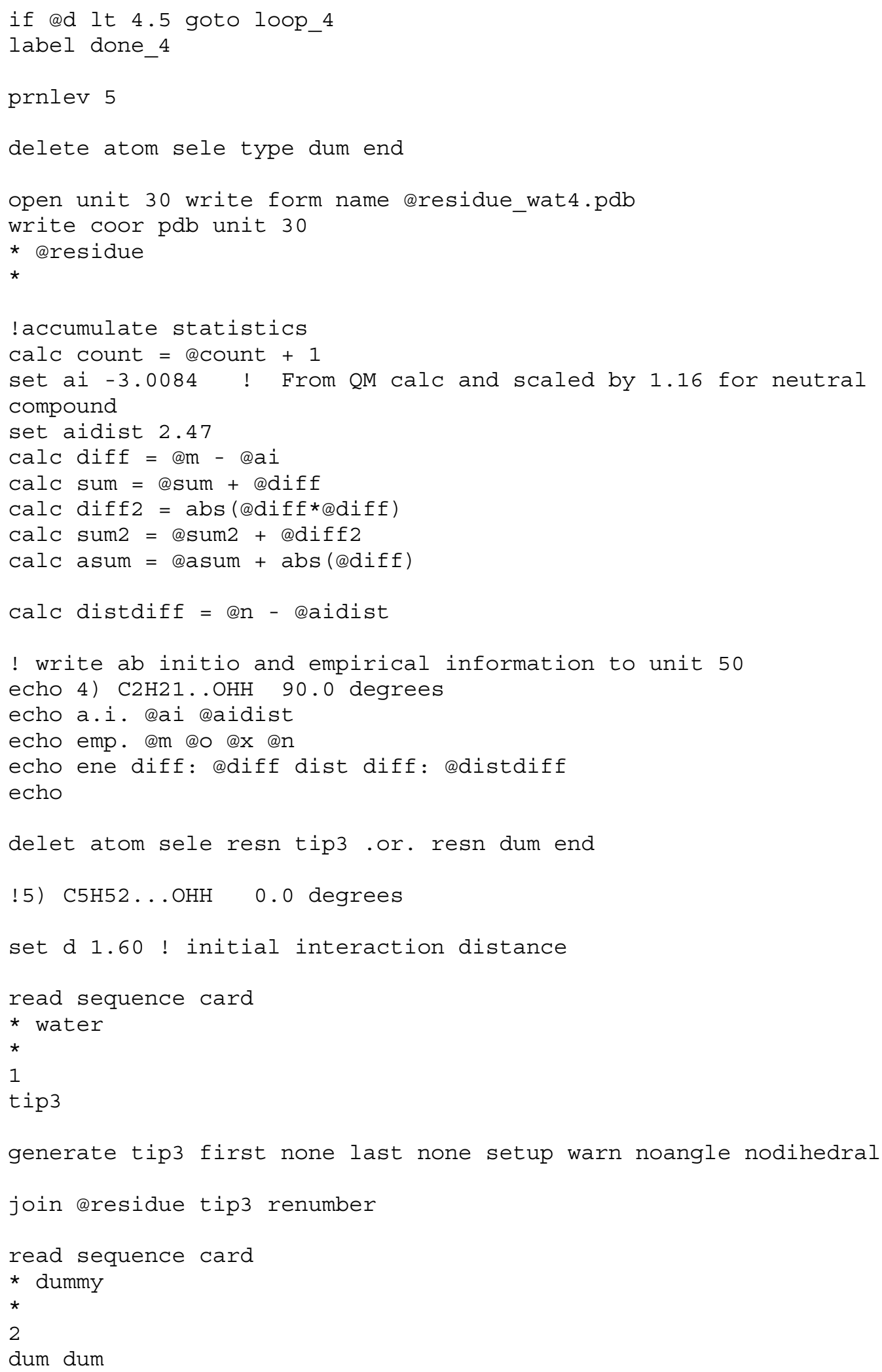




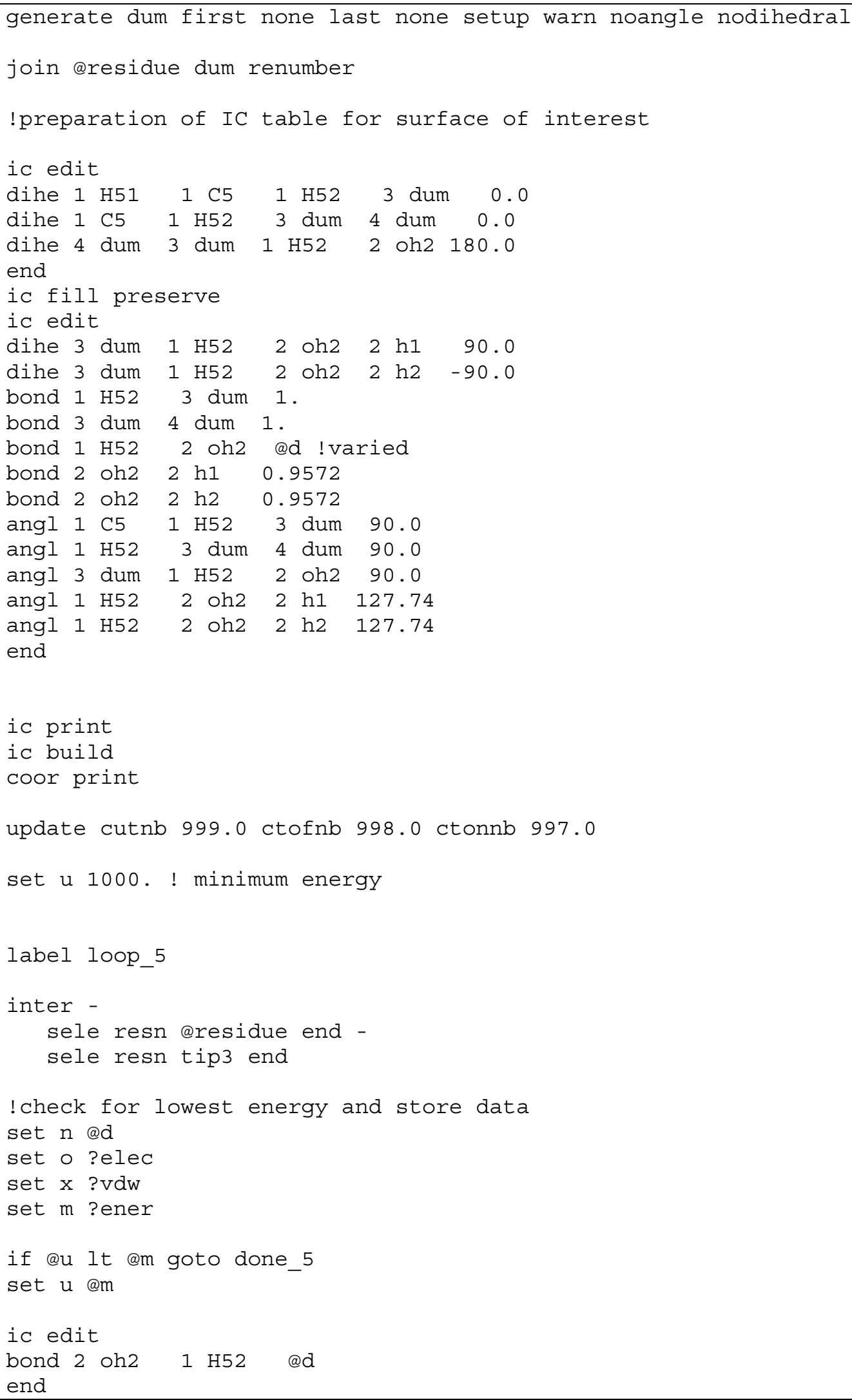




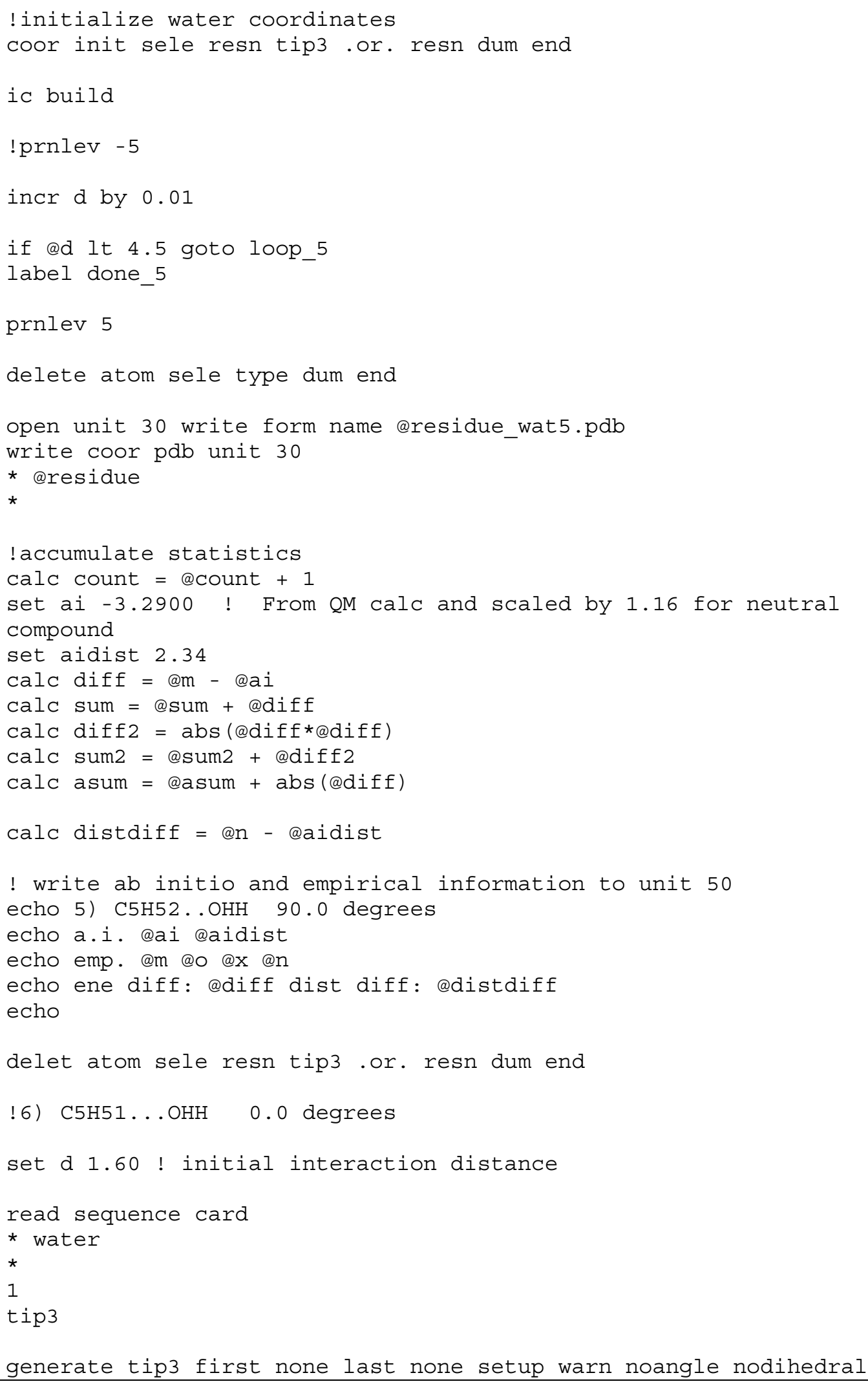




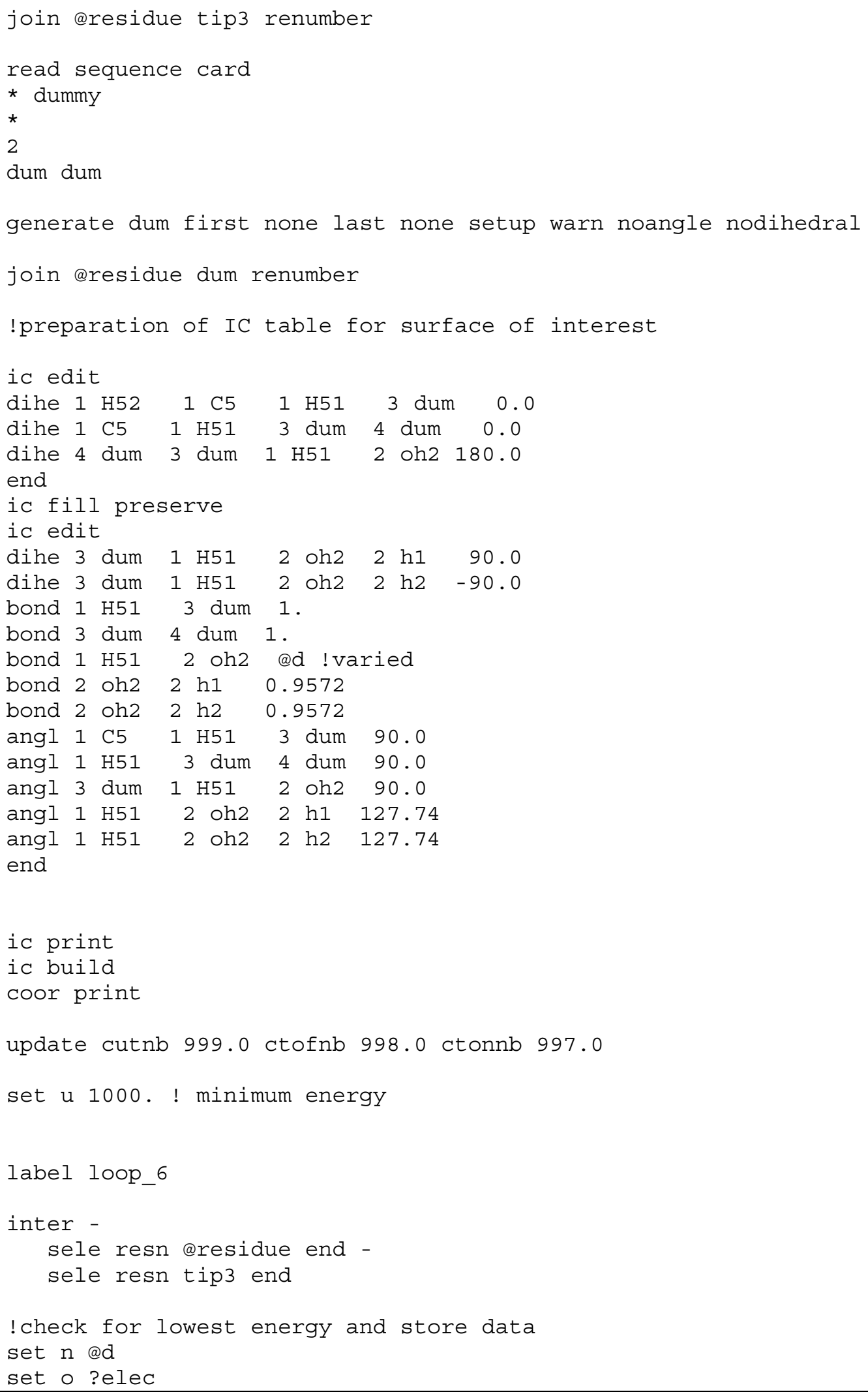




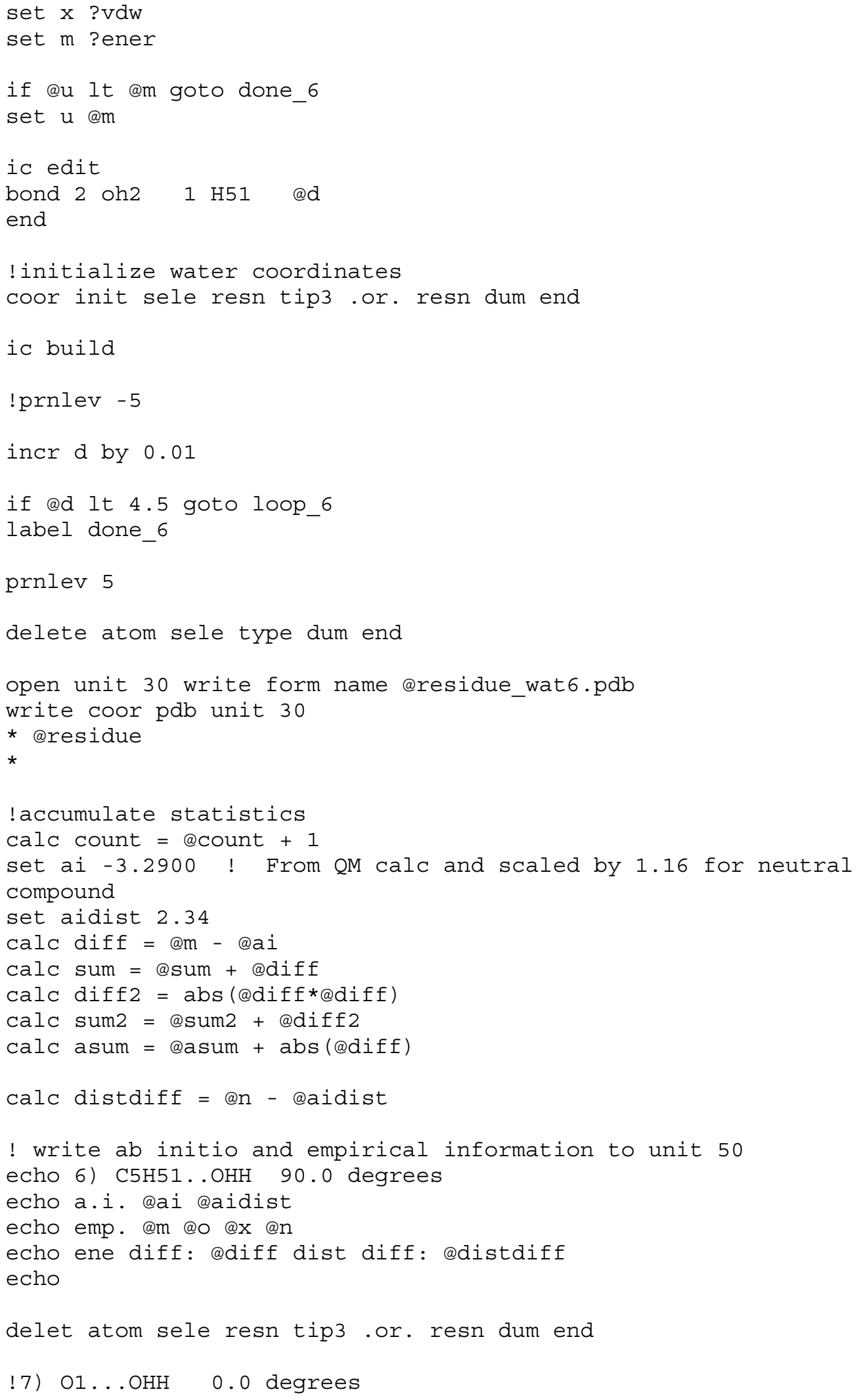




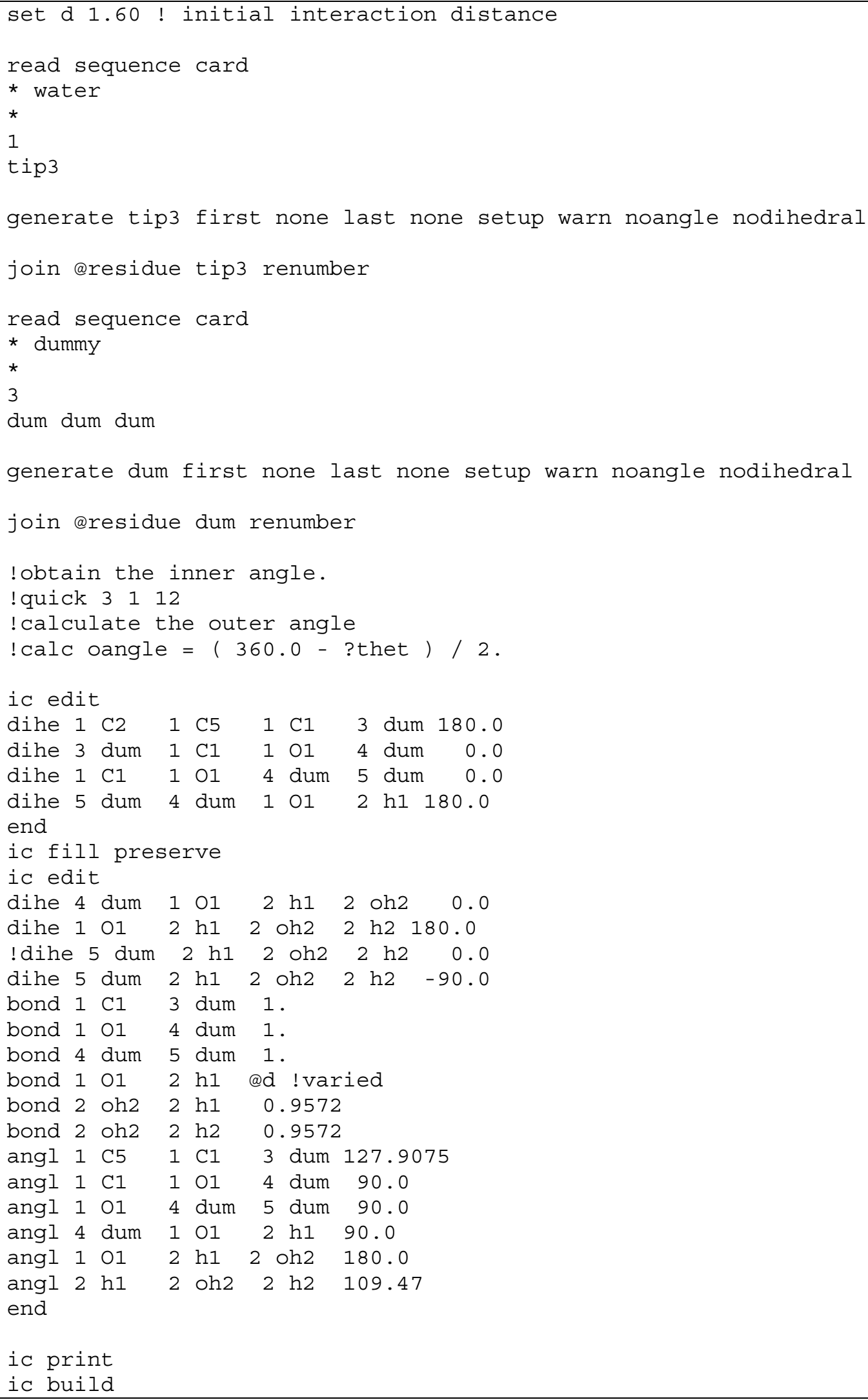




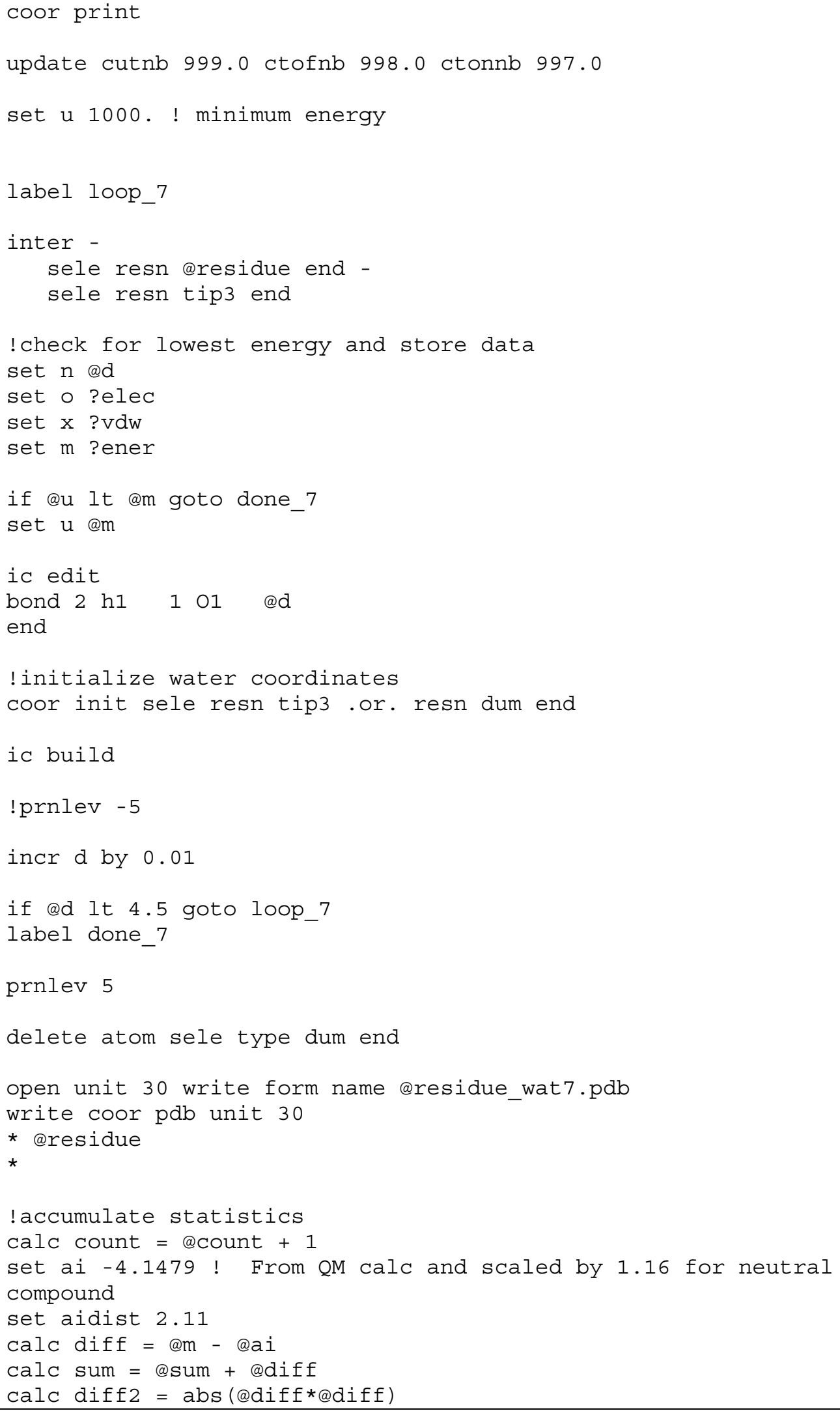




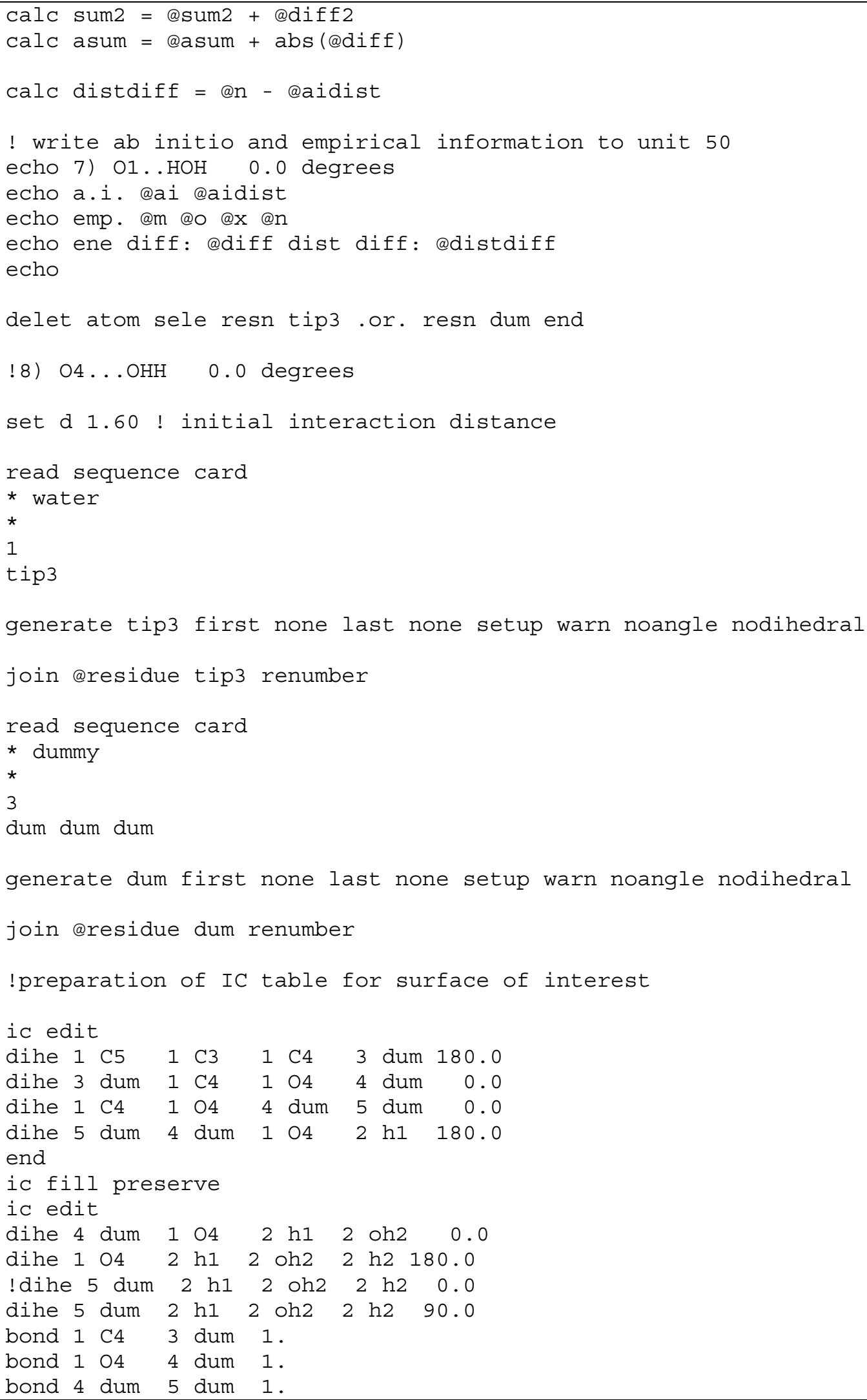




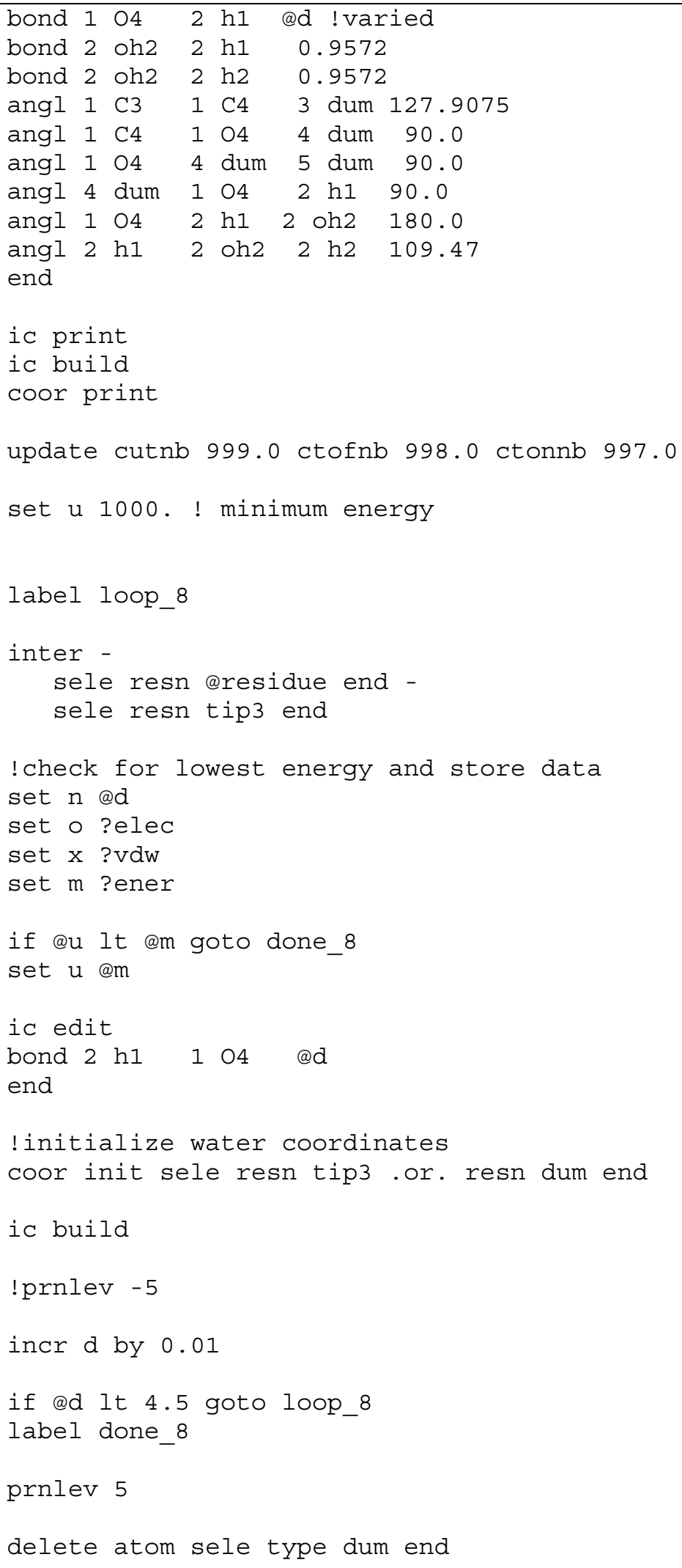




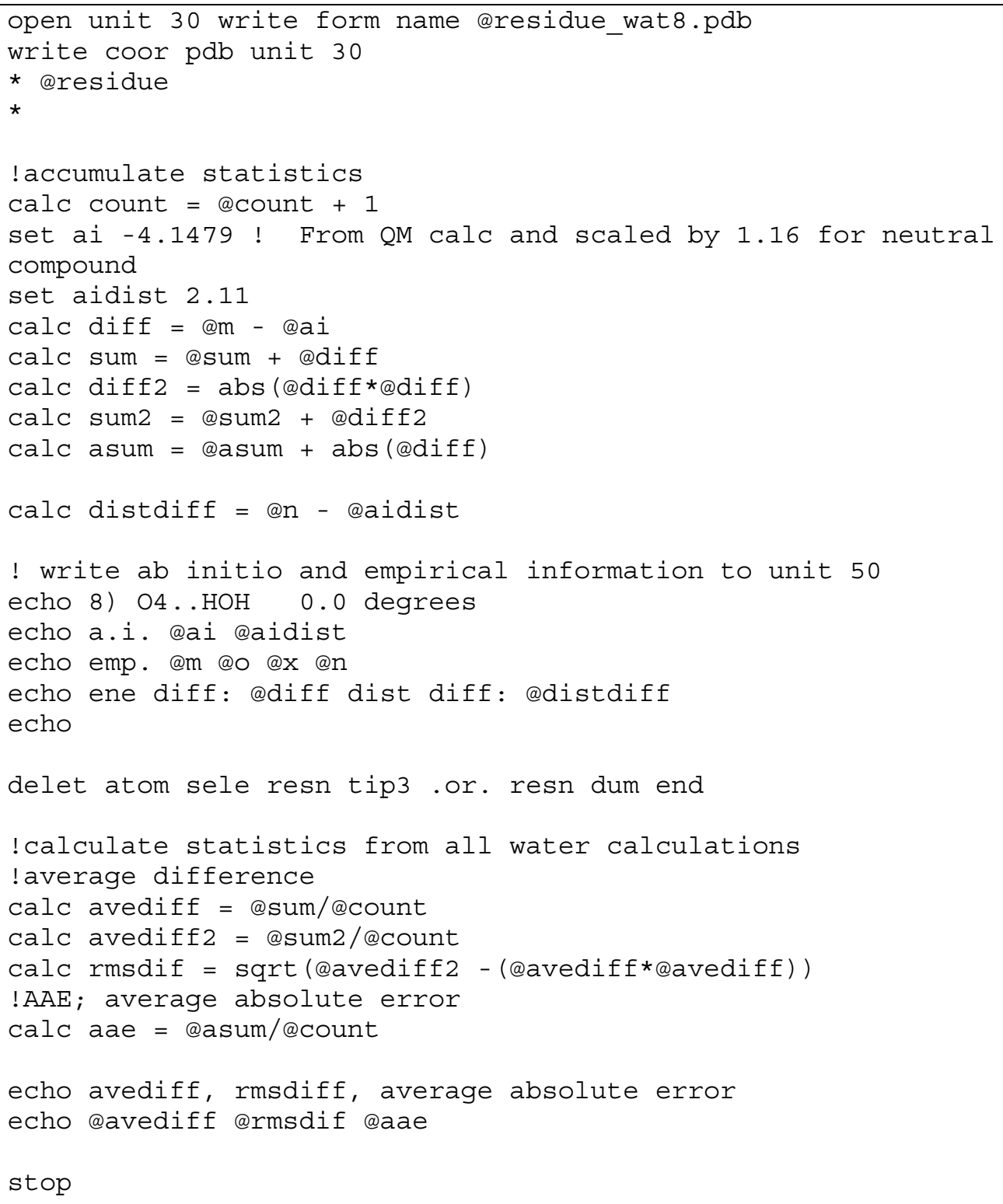

Table 32: Parameterization script (MM): Water - CPD dipole and optimal interaction energies and distances

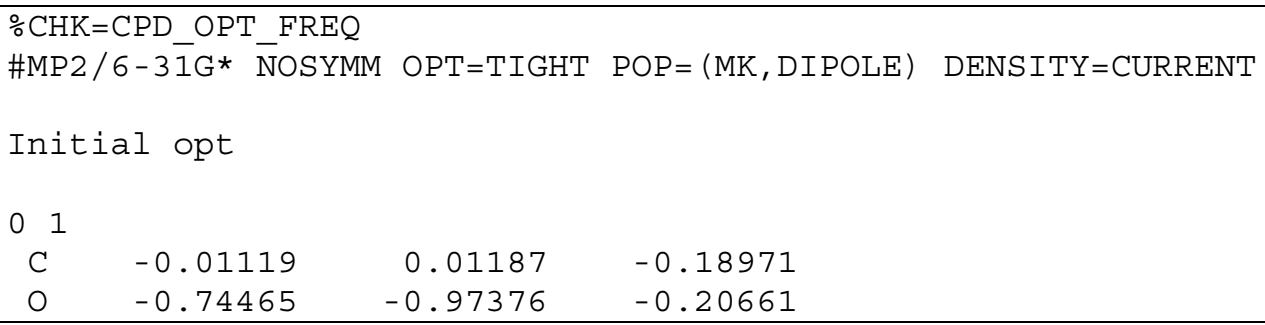




\begin{tabular}{|c|c|c|c|}
\hline $\mathrm{C}$ & 1.51541 & -0.02896 & -0.12172 \\
\hline $\mathrm{H}$ & 1.91154 & -0.52385 & -1.02065 \\
\hline $\mathrm{H}$ & 1.84573 & -0.63344 & 0.73519 \\
\hline $\mathrm{C}$ & 1.98602 & 1.42895 & -0.02109 \\
\hline $\mathrm{H}$ & 2.80533 & 1.65720 & -0.71746 \\
\hline $\mathrm{H}$ & 2.35965 & 1.66407 & 0.98651 \\
\hline $\mathrm{C}$ & 0.76424 & 2.29273 & -0.32771 \\
\hline $\mathrm{O}$ & 0.77831 & 3.51602 & -0.44209 \\
\hline $\mathrm{C}$ & -0.48850 & 1.45196 & -0.17369 \\
\hline $\mathrm{H}$ & -1.19878 & 1.64493 & -0.98696 \\
\hline $\mathrm{H}$ & -0.99637 & 1.68132 & 0.77091 \\
\hline \multicolumn{4}{|c|}{$\begin{array}{l}--\operatorname{LINK} 1-- \\
\text { \%CHK=CPD_OPT_FREQ_MP2 }-2 \\
\# M P 2 / 6-3 \overline{1 G} * \text { NOSYMM GEOM=ALLCHECK FREQ IOP }(7 / 33=1)\end{array}$} \\
\hline \multicolumn{4}{|c|}{$\begin{array}{l}\text { Freq calc for force constants to get target normal mode freq and } \\
\text { contribution }\end{array}$} \\
\hline 01 & & & \\
\hline
\end{tabular}

Table 33: Frequency calculation script for force constants to get target normal mode frequency and percent contribution

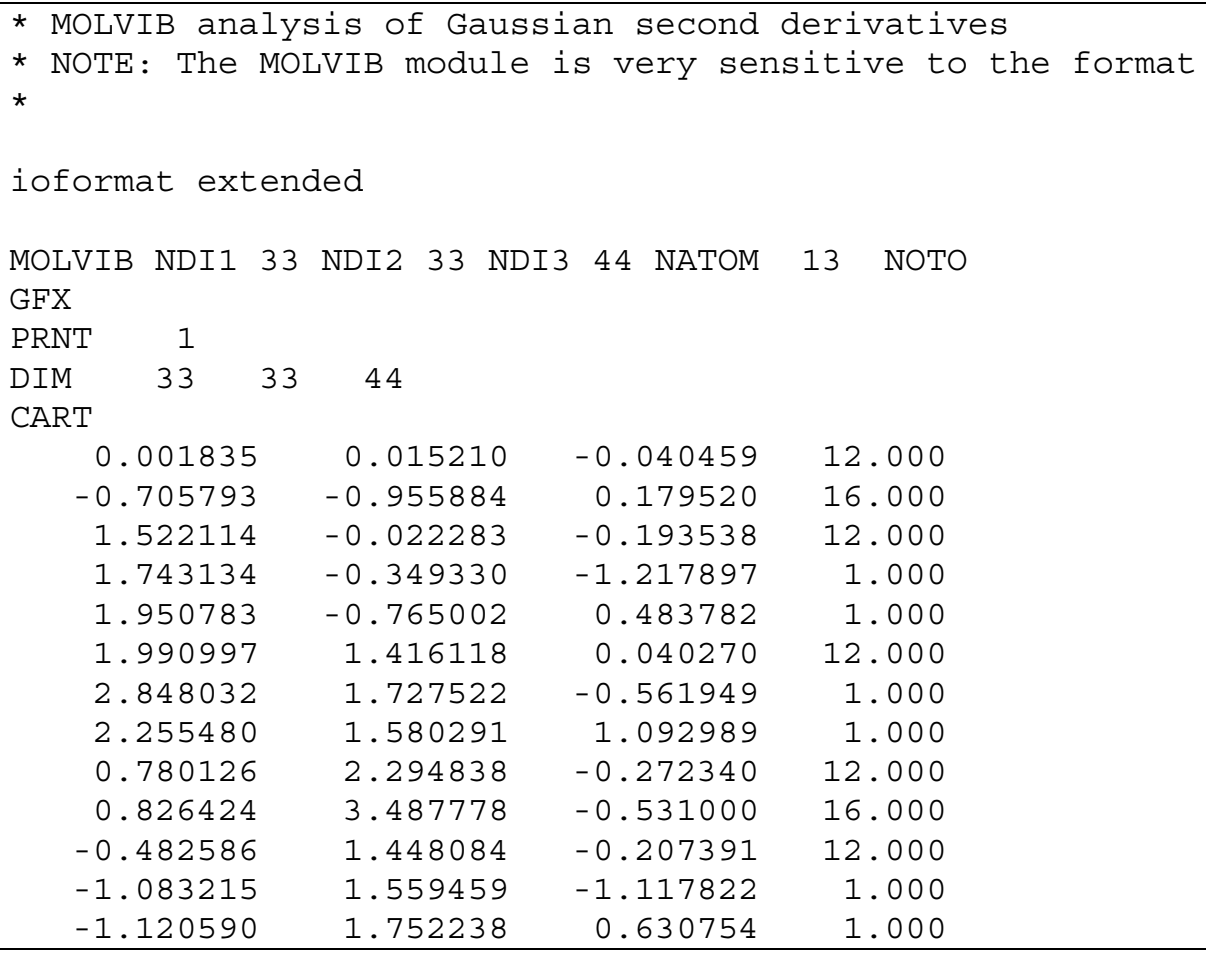




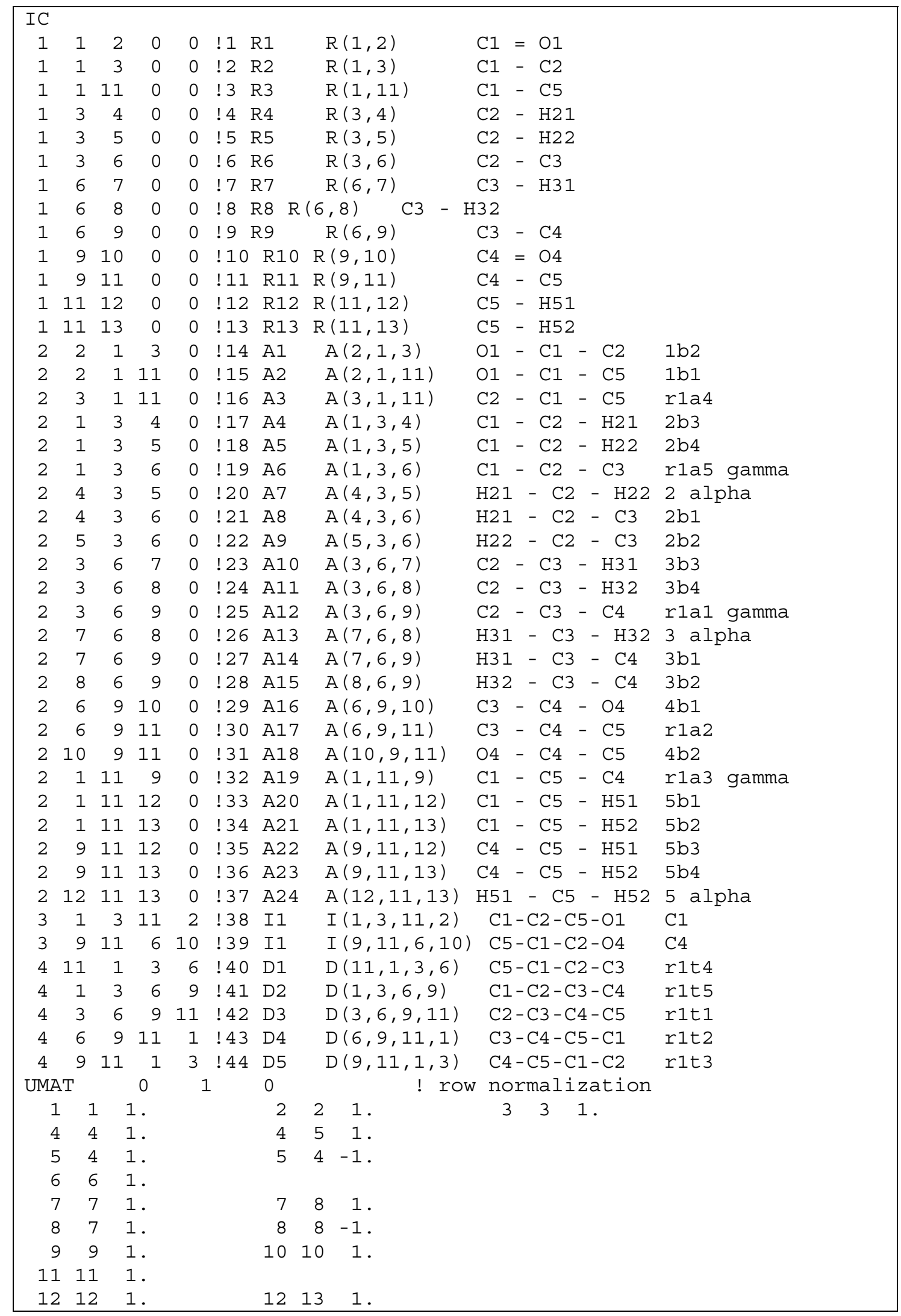




\begin{tabular}{|c|c|c|c|c|c|c|c|c|c|c|c|}
\hline 13 & 12 & 1. & 13 & $\begin{array}{ll}313 \\
\end{array}$ & -1. & & & & & & \\
\hline 14 & 19 & 1. & 14 & $4 \quad 20$ & 5. & & & & & & \\
\hline 15 & 17 & 1. & 15 & $5 \quad 18$ & -1 & 15 & 21 & 1. & 15 & 22 & -1 \\
\hline 16 & 17 & -1 & 16 & $6 \quad 18$ & -1 & 16 & 21 & 1. & 16 & 22 & 1. \\
\hline 17 & 17 & -1. & 17 & $7 \quad 18$ & 1. & 17 & 21 & 1. & 17 & 22 & -1 \\
\hline 18 & 25 & 1. & 18 & $8 \quad 26$ & 5. & & & & & & \\
\hline 19 & 23 & 1. & 19 & 924 & -1. & 19 & 27 & 1. & 19 & 28 & -1 \\
\hline 20 & 23 & -1 & 20 & b 24 & -1. & 20 & 27 & 1. & 20 & 28 & 1. \\
\hline 21 & 23 & -1 & 21 & 124 & 1. & 21 & 27 & 1. & 21 & 28 & -1 \\
\hline 22 & 32 & 1. & 22 & 237 & 5. & & & & & & \\
\hline 23 & 33 & 1. & 23 & 34 & -1. & 23 & 35 & 1. & 23 & 36 & -1 \\
\hline 24 & 33 & 1. & 24 & $4 \quad 34$ & 1. & 24 & 35 & -1. & 24 & 36 & -1 \\
\hline 25 & 33 & 1. & 25 & $5 \quad 34$ & -1. & 25 & 35 & -1. & 25 & 36 & 1. \\
\hline 26 & 38 & 1. & & & & & & & & & \\
\hline 27 & 15 & 1. & 27 & $7 \quad 14$ & -1. & & & & & & \\
\hline 28 & 39 & 1. & & & & & & & & & \\
\hline 29 & 29 & 1. & 29 & $\begin{array}{ll}9 & 31\end{array}$ & -1. & & & & & & \\
\hline 30 & 25 & 1. & 30 & $0 \quad 30$ & -0.81 & 30 & 32 & 0.31 & 30 & 16 & 0.31 \\
\hline 30 & 19 & -0.81 & & & & & & & & & \\
\hline 31 & 30 & -1.12 & 31 & 132 & 1.81 & 31 & 16 & -1.81 & 31 & 19 & 1.12 \\
\hline 32 & 42 & 0.31 & 32 & 243 & -0.81 & 32 & 44 & 1 & 32 & 40 & -0.81 \\
\hline 32 & 41 & 0.31 & & & & & & & & & \\
\hline 33 & 42 & -1.81 & 33 & $\begin{array}{l}343 \\
\end{array}$ & 1.12 & 33 & 40 & -1.12 & 33 & 41 & 1.81 \\
\hline \multicolumn{12}{|c|}{-1} \\
\hline PED & & 0 & 15 & & & & & & & & \\
\hline 1 & & $1=01$ & & & & & & & & & \\
\hline 2 & & $1-\mathrm{C} 2$ & & & & & & & & & \\
\hline 3 & & $1-C 5$ & & & & & & & & & \\
\hline 4 & & $2-\mathrm{H} 2 \mathrm{sy}$ & & & & & & & & & \\
\hline 5 & & $2-\mathrm{H} 2 \mathrm{as}$ & & & & & & & & & \\
\hline 6 & & $2-C 3$ & & & & & & & & & \\
\hline 7 & & $3-\mathrm{H} 3 \mathrm{sy}$ & & & & & & & & & \\
\hline 8 & & $3-\mathrm{H} 3 \mathrm{as}$ & & & & & & & & & \\
\hline 9 & & $3-C 4$ & & & & & & & & & \\
\hline 10 & & $4=04$ & & & & & & & & & \\
\hline 11 & & $4-C 5$ & & & & & & & & & \\
\hline 12 & & $5-H 5 s y$ & & & & & & & & & \\
\hline 13 & & $5-\mathrm{H} 5 \mathrm{as}$ & & & & & & & & & \\
\hline 14 & & $-\mathrm{C} 2 \mathrm{H} 2$ & & & & & & & & & \\
\hline 15 & & $\mathrm{C} 2 \mathrm{H} 2$ & & & & & & & & & \\
\hline 16 & & $\mathrm{C} 2 \mathrm{H} 2$ & & & & & & & & & \\
\hline 17 & & $\mathrm{C} 2 \mathrm{H} 2$ & & & & & & & & & \\
\hline 18 & & $-\mathrm{C} 3 \mathrm{H} 2$ & & & & & & & & & \\
\hline 19 & & $\mathrm{C} 3 \mathrm{H} 2$ & & & & & & & & & \\
\hline 20 & & $\mathrm{C} 3 \mathrm{H} 2$ & & & & & & & & & \\
\hline 21 & & $\mathrm{C} 3 \mathrm{H} 2$ & & & & & & & & & \\
\hline 22 & & $-\mathrm{C} 5 \mathrm{H} 2$ & & & & & & & & & \\
\hline 23 & & C5H2 & & & & & & & & & \\
\hline 24 & & $\mathrm{C} 5 \mathrm{H} 2$ & & & & & & & & & \\
\hline 25 & & $\mathrm{C} 5 \mathrm{H} 2$ & & & & & & & & & \\
\hline 26 & & $\mathrm{C} 1=\mathrm{O} 1$ & & & & & & & & & \\
\hline 27 & & $\mathrm{C} 1=\mathrm{O} 1$ & & & & & & & & & \\
\hline 28 & & $C 4=04$ & & & & & & & & & \\
\hline 29 & & $C 4=04$ & & & & & & & & & \\
\hline 30 & & 5RNGsY & & & & & & & & & \\
\hline
\end{tabular}




\begin{tabular}{|c|c|c|c|c|}
\hline d-5RNGas & & & & \\
\hline $32 t$-5RNGsy & & & & \\
\hline$t-5$ RNGas & & & & \\
\hline-1 & & & & \\
\hline FMAT 1 & 0 & Gaussian FX mat & Erix is in inpu & \\
\hline orientation & & & & \\
\hline Force constants ir & n Cartesian co & ordinates: & & \\
\hline 1 & 2 & 3 & 4 & \\
\hline 5 & & & & \\
\hline $0.643611 D+00$ & & & & \\
\hline $0.236491 D+00$ & $0.805451 D+00$ & & & \\
\hline$-0.770802 \mathrm{D}-01$ & $-0.108522 \mathrm{D}+00$ & $0.263264 \mathrm{D}+00$ & & \\
\hline$-0.318400 D+00$ & $-0.293306 \mathrm{D}+00$ & $0.705900 \mathrm{D}-01$ & $0.332572 \mathrm{D}+00$ & \\
\hline $5-0.296109 \mathrm{D}+00$ & $-0.506491 D+00$ & $0.983692 \mathrm{D}-01$ & $0.344365 \mathrm{D}+00$ & \\
\hline $0.553658 \mathrm{D}+00$ & & & & \\
\hline $\begin{array}{ll}6 & 0.692472 \mathrm{D}-01\end{array}$ & $0.967589 \mathrm{D}-01$ & $-0.871856 \mathrm{D}-01$ & $-0.832153 \mathrm{D}-01$ & - \\
\hline $0.115810 \mathrm{D}+00$ & & & & \\
\hline $7-0.169439 \mathrm{D}+00$ & $0.318536 \mathrm{D}-01$ & $0.368375 D-02$ & $-0.296714 \mathrm{D}-01$ & - \\
\hline $0.215520 \mathrm{D}-01$ & & & & \\
\hline $80.173909 \mathrm{D}-01$ & $-0.881021 D-01$ & $-0.136055 \mathrm{D}-02$ & $-0.421041 \mathrm{D}-01$ & \\
\hline $0.352002 \mathrm{D}-02$ & & & & \\
\hline $9 \quad 0.348225 \mathrm{D}-02$ & $0.172581 \mathrm{D}-02$ & $-0.893633 \mathrm{D}-01$ & $0.108227 \mathrm{D}-01$ & \\
\hline $0.510280 \mathrm{D}-02$ & & & & \\
\hline $10-0.733210 \mathrm{D}-02$ & $0.801079 D-02$ & $0.366843 \mathrm{D}-01$ & $-0.464062 \mathrm{D}-03$ & - \\
\hline $0.372434 \mathrm{D}-03$ & & & & \\
\hline $110.634848 \mathrm{D}-03$ & $-0.134744 \mathrm{D}-02$ & $0.116934 \mathrm{D}-02$ & $-0.106853 \mathrm{D}-02$ & - \\
\hline $0.118202 \mathrm{D}-03$ & & & & \\
\hline $120.140842 \mathrm{D}-02$ & $-0.321319 D-03$ & $-0.252530 \mathrm{D}-02$ & $-0.487130 \mathrm{D}-04$ & - \\
\hline $0.259966 \mathrm{D}-03$ & & & & \\
\hline $13-0.134787 \mathrm{D}-01$ & $0.252618 \mathrm{D}-01$ & $-0.242071 D-01$ & $0.493770 \mathrm{D}-02$ & - \\
\hline $0.129191 D-02$ & & & & \\
\hline $140.203779 \mathrm{D}-02$ & $-0.184045 \mathrm{D}-02$ & $-0.148578 \mathrm{D}-02$ & $-0.123142 \mathrm{D}-02$ & \\
\hline $0.667746 \mathrm{D}-03$ & & & & \\
\hline $150.554168 \mathrm{D}-02$ & $-0.403406 \mathrm{D}-02$ & $0.287438 \mathrm{D}-02$ & $-0.873699 \mathrm{D}-03$ & - \\
\hline $0.698227 \mathrm{D}-03$ & & & & \\
\hline $16-0.166763 \mathrm{D}-01$ & $-0.325877 \mathrm{D}-01$ & $-0.642492 \mathrm{D}-02$ & $-0.776981 D-02$ & - \\
\hline $0.149683 \mathrm{D}-03$ & & & & \\
\hline $17-0.184391 \mathrm{D}-01$ & $-0.620067 \mathrm{D}-02$ & $0.154042 \mathrm{D}-02$ & $0.268947 \mathrm{D}-02$ & \\
\hline $0.124631 \mathrm{D}-02$ & & & & \\
\hline $180.460571 D-03$ & $0.600984 \mathrm{D}-02$ & $0.266769 \mathrm{D}-03$ & $0.368527 \mathrm{D}-02$ & \\
\hline $0.520298 \mathrm{D}-04$ & & & & \\
\hline $19-0.122688 \mathrm{D}-02$ & $-0.256346 \mathrm{D}-03$ & $0.676854 \mathrm{D}-04$ & $-0.841820 \mathrm{D}-04$ & - \\
\hline $0.325315 \mathrm{D}-03$ & & & & \\
\hline $20 \quad 0.286359 \mathrm{D}-02$ & $-0.684261 \mathrm{D}-02$ & $-0.673492 \mathrm{D}-03$ & $-0.348106 \mathrm{D}-02$ & \\
\hline $0.497678 \mathrm{D}-04$ & & & & \\
\hline $21-0.141565 \mathrm{D}-02$ & $-0.179596 \mathrm{D}-02$ & $0.108578 D-03$ & $-0.811366 \mathrm{D}-03$ & - \\
\hline $0.251657 \mathrm{D}-03$ & & & & \\
\hline $22-0.350794 \mathrm{D}-03$ & $-0.209015 D-03$ & $-0.858540 \mathrm{D}-03$ & $-0.188155 \mathrm{D}-03$ & - \\
\hline $0.230343 \mathrm{D}-03$ & & & & \\
\hline $230.164796 \mathrm{D}-02$ & $-0.142444 \mathrm{D}-02$ & $-0.853379 D-03$ & $-0.795777 D-03$ & - \\
\hline $0.281608 \mathrm{D}-05$ & & & & \\
\hline $240.415373 \mathrm{D}-03$ & $-0.469615 \mathrm{D}-03$ & $-0.338904 D-03$ & $-0.286168 D-03$ & \\
\hline $0.536667 \mathrm{D}-04$ & & & & \\
\hline $250.265346 \mathrm{D}-01$ & $-0.248586 \mathrm{D}-01$ & $0.217737 \mathrm{D}-02$ & $-0.553549 \mathrm{D}-02$ & \\
\hline $0.167721 \mathrm{D}-01$ & & & & \\
\hline
\end{tabular}




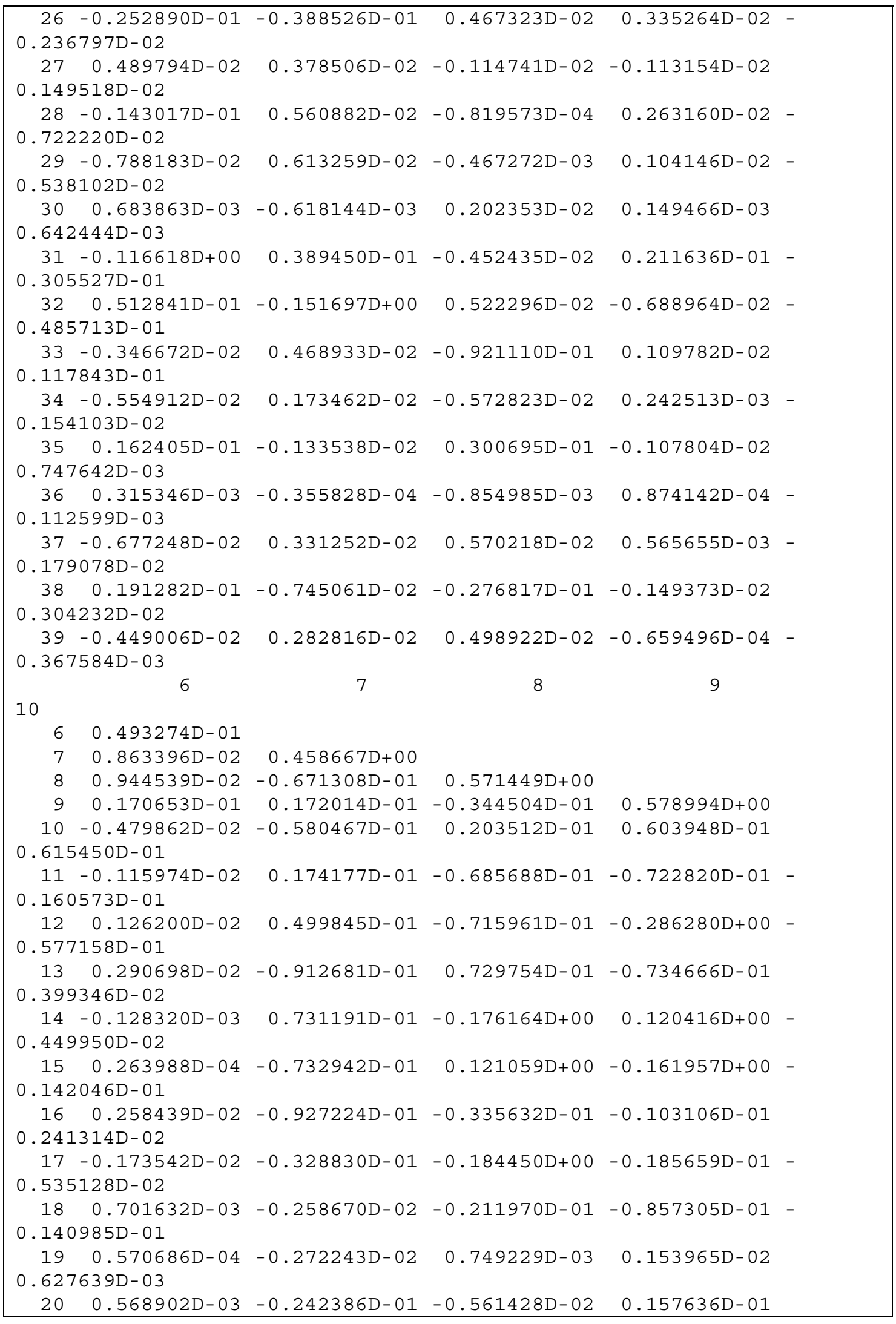




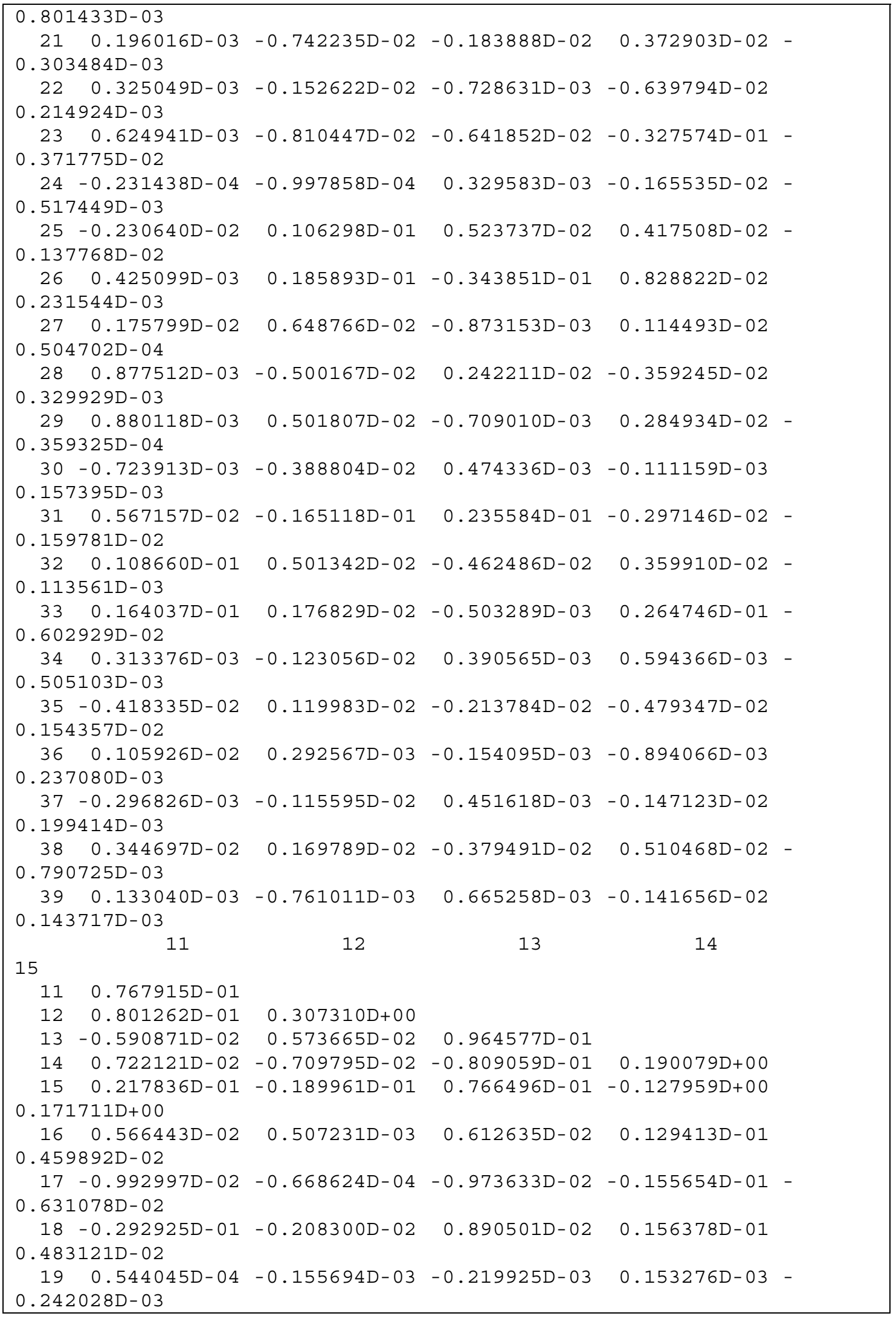




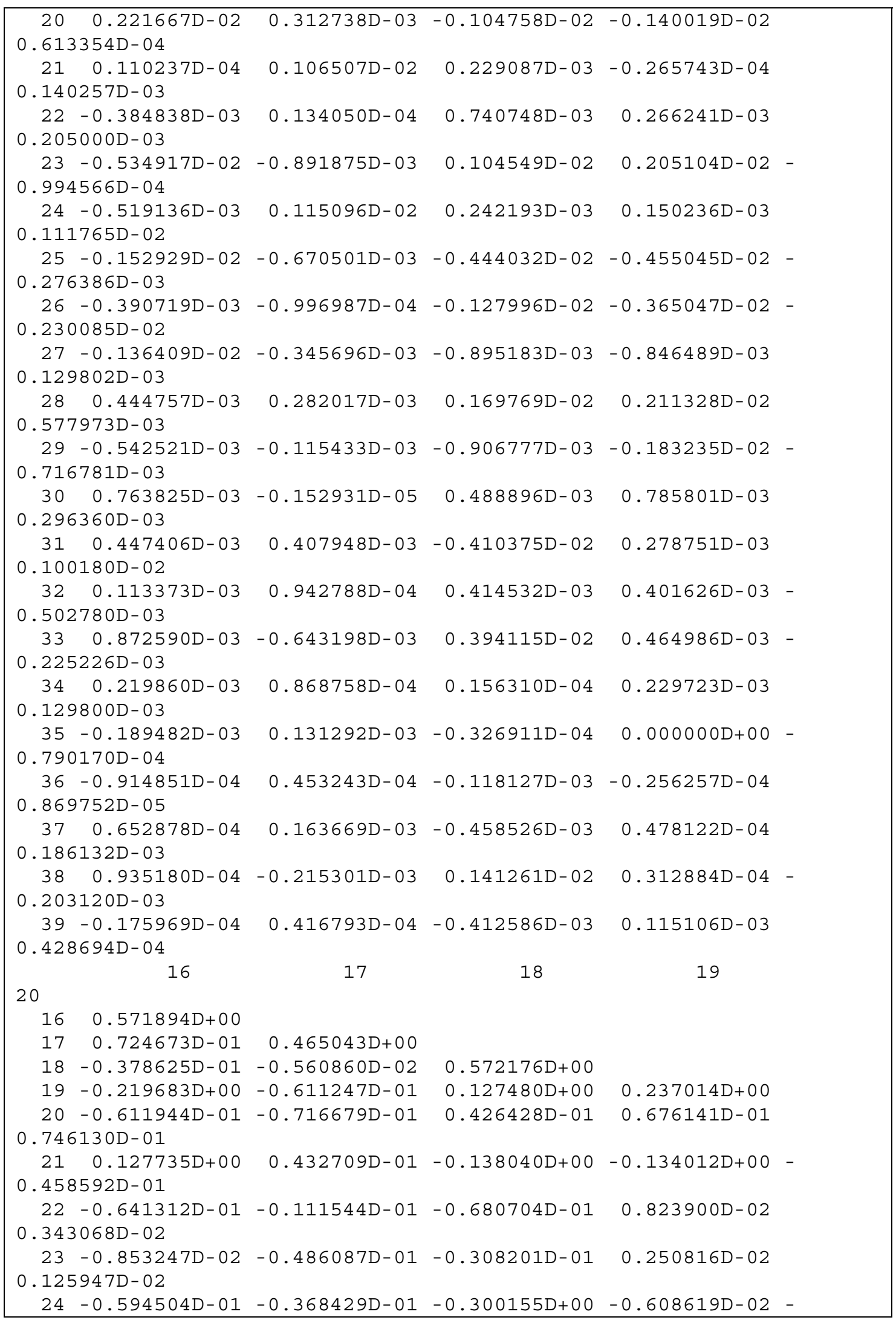




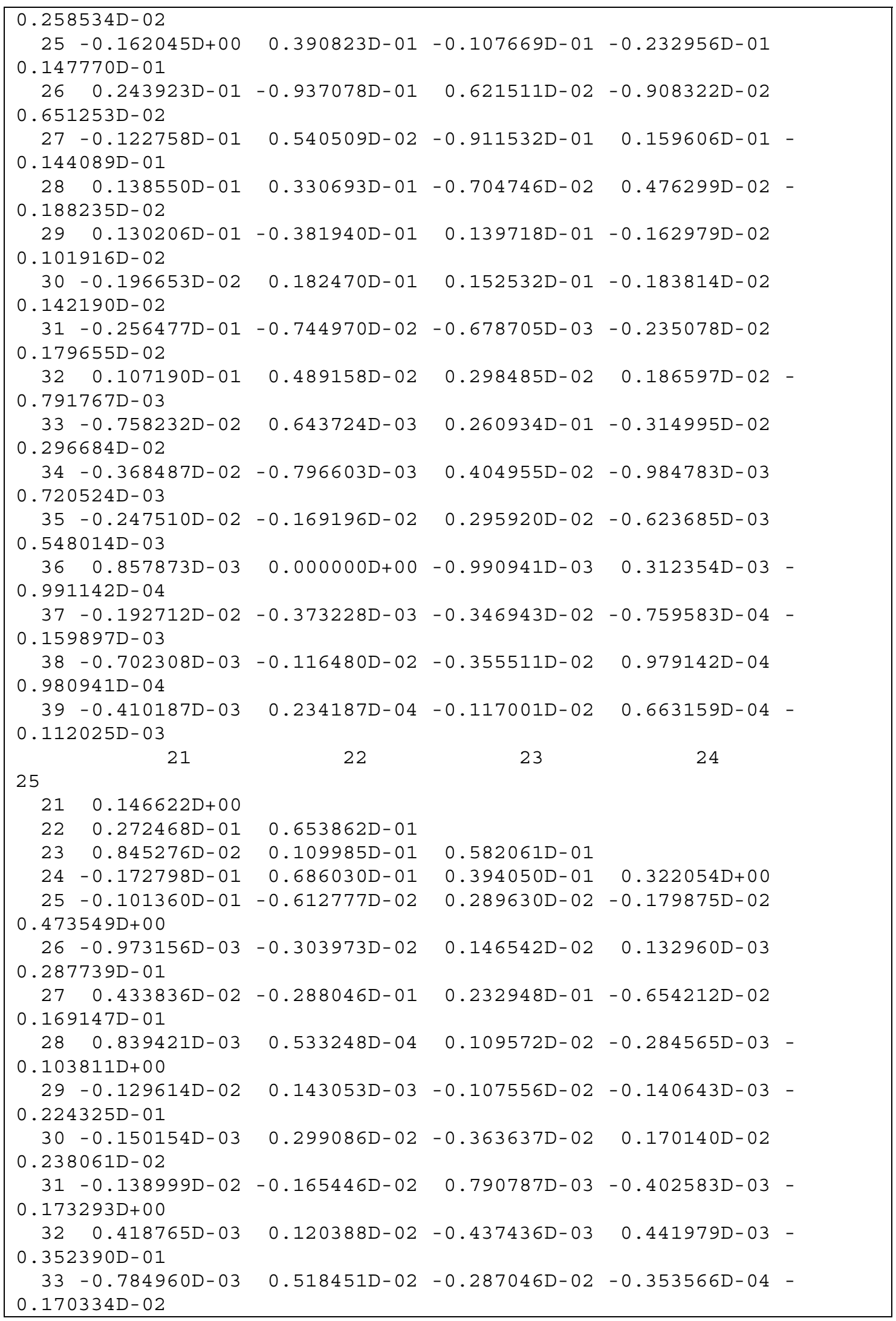




\begin{tabular}{|c|c|c|c|c|}
\hline \multicolumn{5}{|l|}{$\begin{array}{l}34-0.404923 \mathrm{D}-03 \\
0.159136 \mathrm{D}-01\end{array}$} \\
\hline $35-0.134071 D-03$ & $0.476723 \mathrm{D}-03$ & $-0.216469 \mathrm{D}-03$ & $-0.145765 \mathrm{D}-04$ & - \\
\hline \multicolumn{5}{|l|}{$0.913023 \mathrm{D}-02$} \\
\hline \multicolumn{4}{|l|}{$0.341239 \mathrm{D}-02$} & \\
\hline \multicolumn{5}{|l|}{$0.148733 \mathrm{D}-01$} \\
\hline $\begin{array}{l}380.221867 D-04 \\
0.979896 D-02\end{array}$ & \multicolumn{4}{|c|}{$0.979896 \mathrm{D}-02$} \\
\hline \multicolumn{5}{|c|}{$0.140187 \mathrm{D}-02$} \\
\hline \multicolumn{4}{|l|}{ 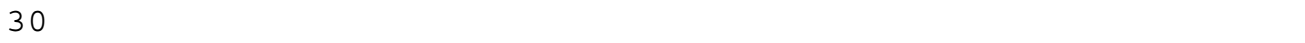 } & \\
\hline $0.963939 D+00$ & & & & \\
\hline $27-0.155551 D+00$ & $0.274839 \mathrm{D}+00$ & & & \\
\hline $28-0.250020 D-01$ & $0.237532 \mathrm{D}-02$ & $0.821285 \mathrm{D}-01$ & & \\
\hline $29-0.712441 D+00$ & $0.141470 D+00$ & $0.286371 D-01$ & $0.793657 \mathrm{D}+00$ & \\
\hline $30 \quad 0.139093 \mathrm{D}+00$ & $-0.958204 \mathrm{D}-01$ & $-0.128779 D-02$ & $-0.166675 D+00$ & \\
\hline \multicolumn{5}{|l|}{$0.597674 \mathrm{D}-01$} \\
\hline \multicolumn{5}{|c|}{$0.230323 \mathrm{D}-02$} \\
\hline \multicolumn{5}{|c|}{$0.983449 \mathrm{D}-02$} \\
\hline \multicolumn{5}{|c|}{$0.165987 \mathrm{D}-01$} \\
\hline \multicolumn{5}{|c|}{$0.253504 \mathrm{D}-02$} \\
\hline \multicolumn{5}{|l|}{$0.269836 \mathrm{D}-02$} \\
\hline \multicolumn{5}{|l|}{$0.298439 \mathrm{D}-03$} \\
\hline \multicolumn{5}{|l|}{$0.270887 \mathrm{D}-02$} \\
\hline \multicolumn{5}{|l|}{$0.303236 \mathrm{D}-02$} \\
\hline \multicolumn{5}{|l|}{$0.868158 \mathrm{D}-03$} \\
\hline 31 & 32 & 33 & 34 & \\
\hline \multicolumn{5}{|l|}{35} \\
\hline $31 \quad 0.566030 \mathrm{D}+00$ & & & & \\
\hline $32-0.433064 \mathrm{D}-01$ & $0.452808 D+00$ & & & \\
\hline $330.428433 \mathrm{D}-02$ & $0.632470 \mathrm{D}-02$ & $0.543893 \mathrm{D}+00$ & & \\
\hline $34-0.122989 D+00$ & $0.123682 \mathrm{D}-01$ & $-0.133908 \mathrm{D}+00$ & $0.135731 \mathrm{D}+00$ & \\
\hline $350.147921 \mathrm{D}-01$ & $-0.490156 \mathrm{D}-01$ & $0.269710 \mathrm{D}-01$ & $-0.169360 \mathrm{D}-01$ & \\
\hline \multicolumn{5}{|l|}{$0.476775 \mathrm{D}-01$} \\
\hline \multicolumn{5}{|l|}{$0.254654 \mathrm{D}-01$} \\
\hline \multicolumn{4}{|l|}{$0.518961 \mathrm{D}-02$} & - \\
\hline \multicolumn{5}{|l|}{$0.205644 \mathrm{D}-02$} \\
\hline \multicolumn{5}{|l|}{$0.848745 \mathrm{D}-02$} \\
\hline 36 & 37 & 38 & 39 & \\
\hline
\end{tabular}




\begin{tabular}{|lrrrr|}
\hline 36 & $0.255941 \mathrm{D}+00$ & & & \\
37 & $-0.156444 \mathrm{D}-01$ & $0.147488 \mathrm{D}+00$ & & \\
38 & $0.291891 \mathrm{D}-02$ & $-0.490771 \mathrm{D}-01$ & $0.679189 \mathrm{D}-01$ & \\
39 & $-0.212271 \mathrm{D}-01$ & $-0.135405 \mathrm{D}+00$ & $0.647460 \mathrm{D}-01$ & $0.223940 \mathrm{D}+00$ \\
SCALE & & & \\
0.89 & & & \\
END & & & \\
\end{tabular}

Table 34: Parameterization script (QM): Bond, angle and torsion based on QM calculated force constants for CPD

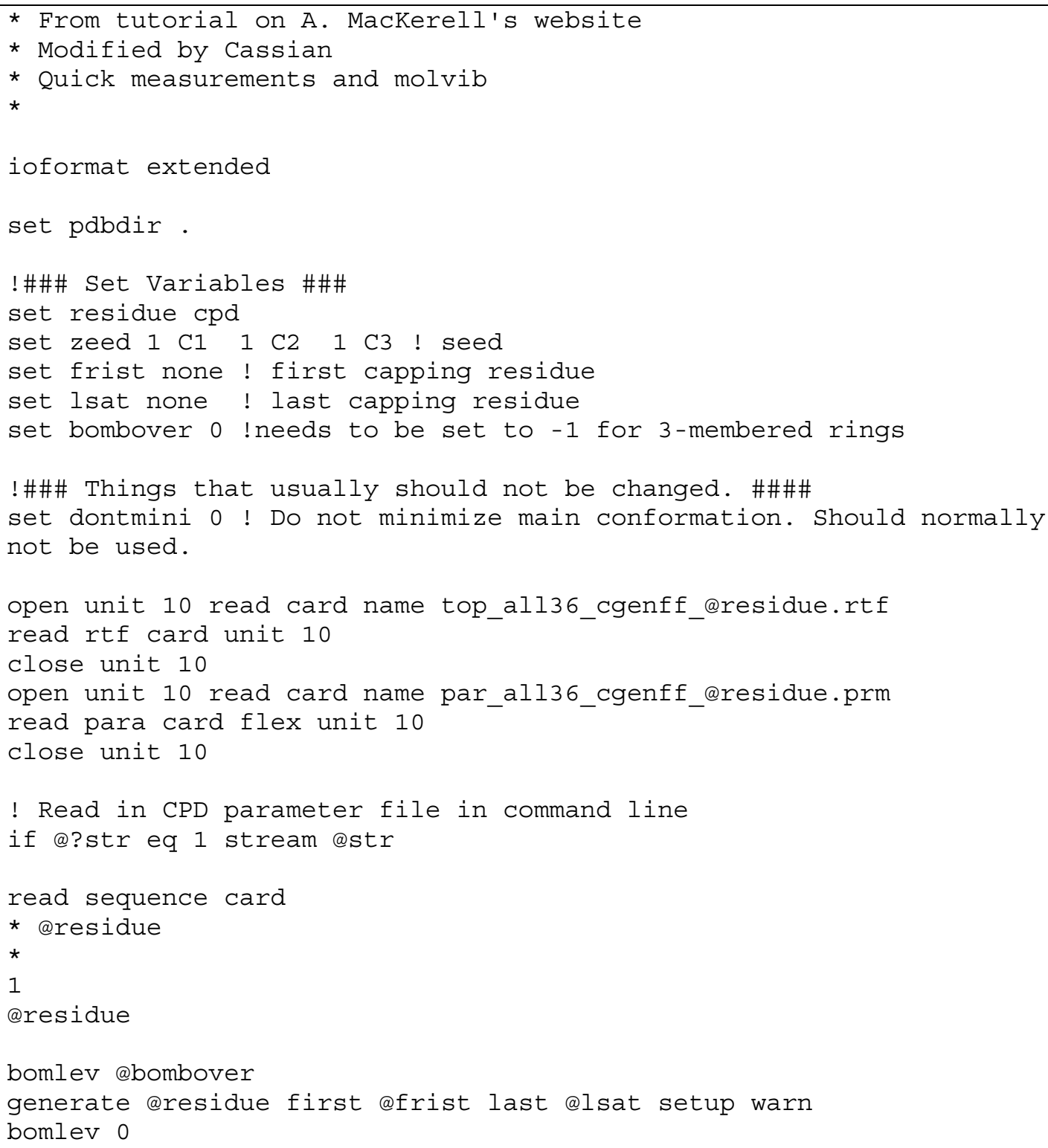




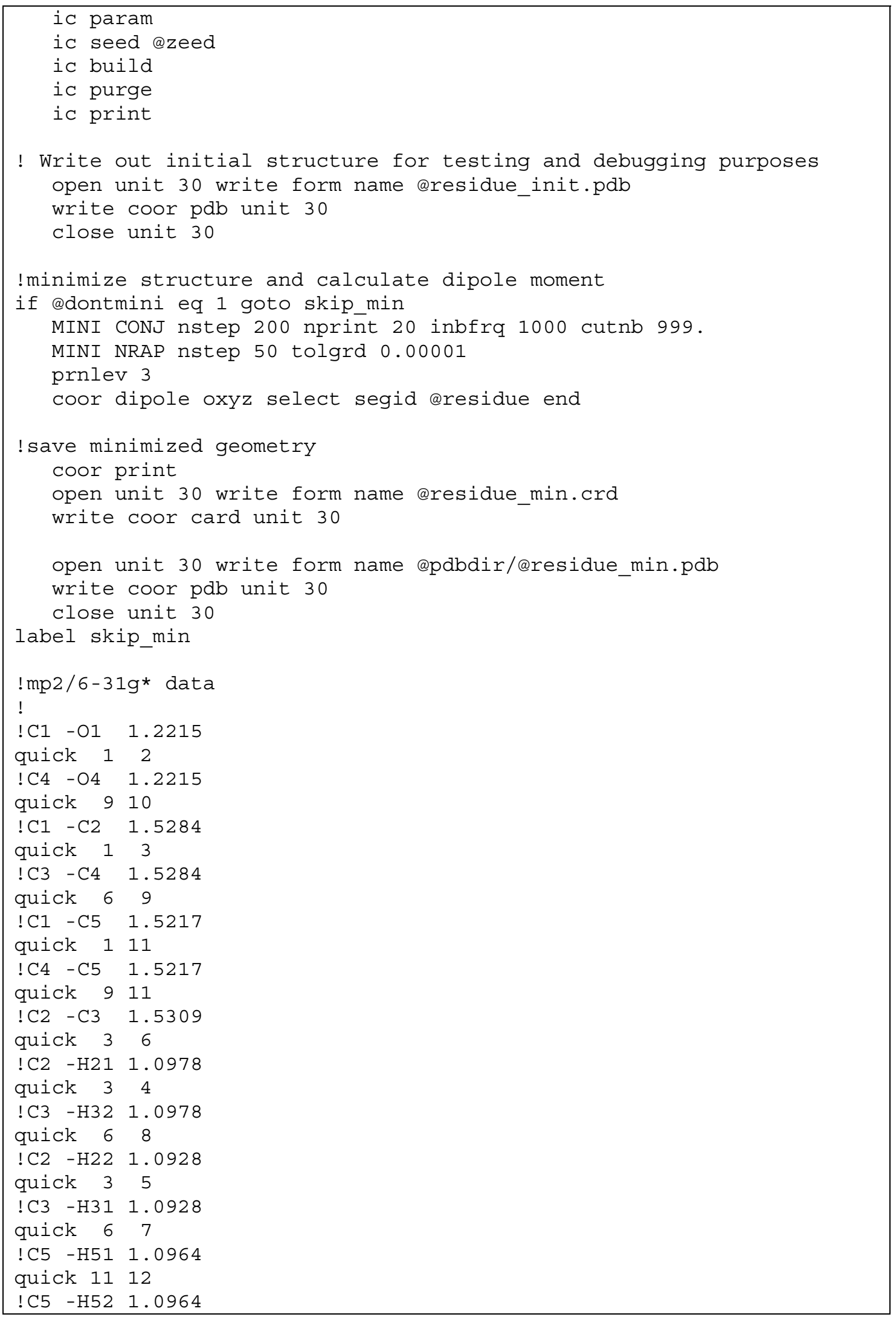




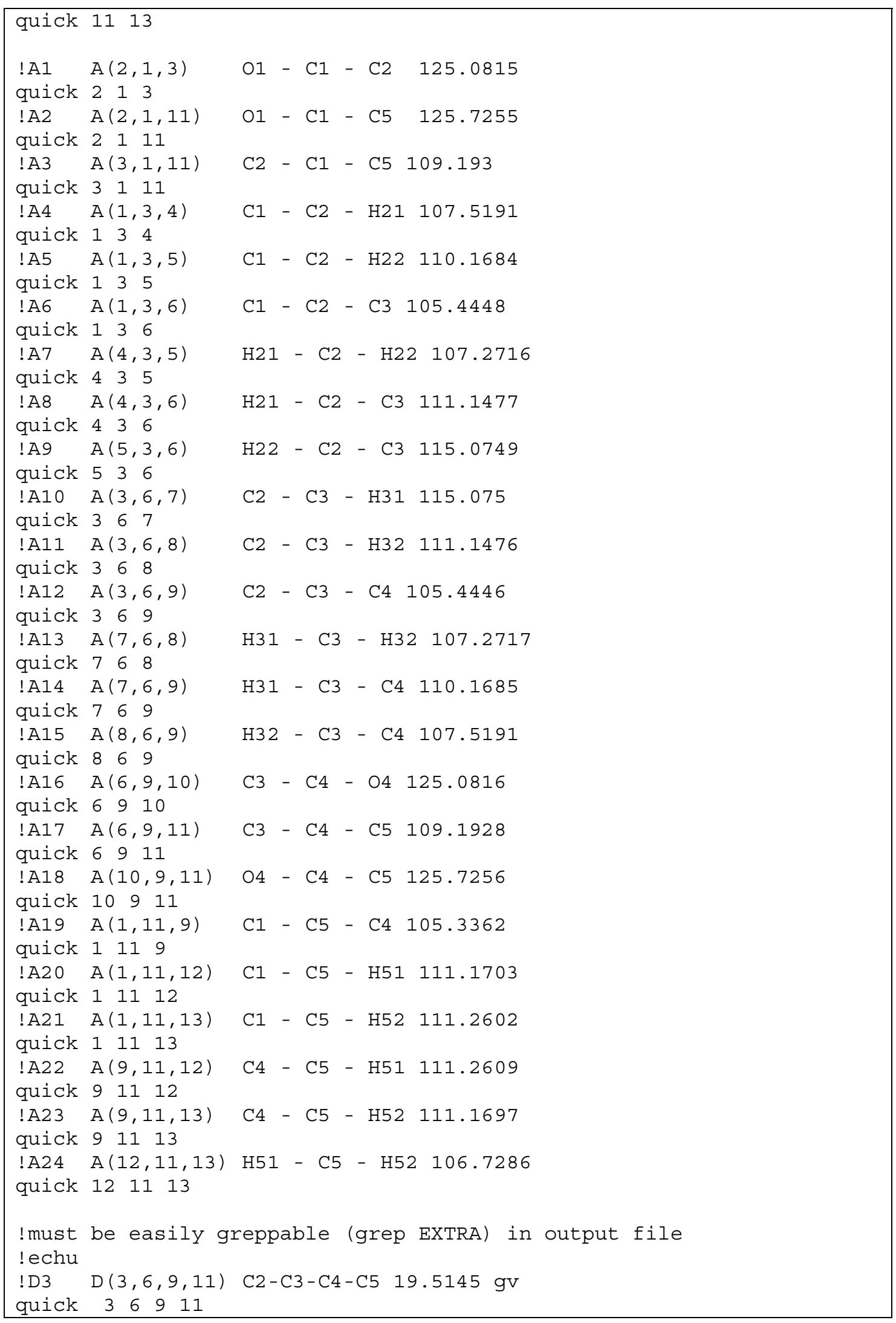




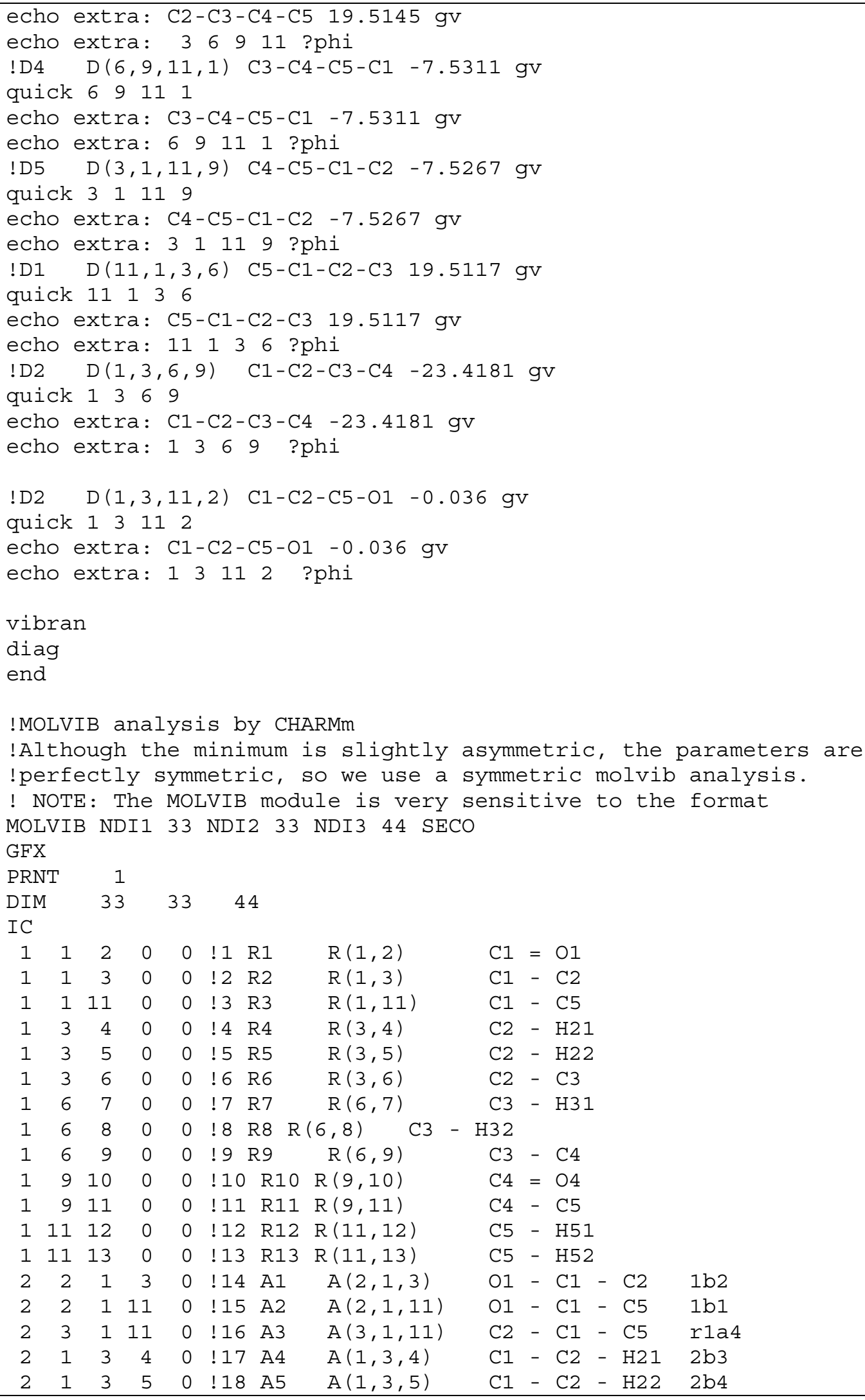




\begin{tabular}{|c|c|c|c|c|c|c|c|c|c|c|c|c|c|c|}
\hline 2 & 1 & 3 & 6 & 0 & $! 19$ & A6 & & $A(1,3,6)$ & $\mathrm{C} 1$ & $-\mathrm{C} 2$ & $-C 3$ & \multicolumn{3}{|c|}{ rla5 gamma } \\
\hline 2 & 4 & 3 & 5 & 0 & $! 20$ & A7 & & $A(4,3,5)$ & H2 1 & $-C$ & $2-\mathrm{H} 22$ & \multicolumn{3}{|c|}{2 alpha } \\
\hline 2 & 4 & 3 & 6 & 0 & !21 & A8 & & $A(4,3,6)$ & H2 1 & $-C$ & $2-C 3$ & \multicolumn{3}{|l|}{$2 \mathrm{~b} 1$} \\
\hline 2 & 5 & 3 & 6 & 0 & $! 22$ & A9 & & $A(5,3,6)$ & H22 & $-C$ & $2-\mathrm{C} 3$ & \multicolumn{3}{|l|}{$2 \mathrm{~b} 2$} \\
\hline 2 & 3 & 6 & 7 & 0 & $! 23$ & A10 & & $A(3,6,7)$ & $\mathrm{C} 2$ & $-\mathrm{C} 3$ & $-\mathrm{H} 3 \mathrm{I}$ & \multicolumn{3}{|l|}{$3 \mathrm{~b} 3$} \\
\hline 2 & 3 & 6 & 8 & 0 & $! 24$ & A11 & & $A(3,6,8)$ & $\mathrm{C} 2$ & $-\mathrm{C} 3$ & $-\mathrm{H} 32$ & \multicolumn{3}{|c|}{$3 \mathrm{~b} 4$} \\
\hline 2 & 3 & 6 & 9 & 0 & $! 25$ & A12 & & $A(3,6,9)$ & $\mathrm{C} 2$ & $-C 3$ & $-\mathrm{C} 4$ & \multicolumn{3}{|c|}{ rlal gamma } \\
\hline 2 & 7 & 6 & 8 & 0 & $! 26$ & A13 & & $A(7,6,8)$ & H31 & $-C$ & $3-$ H32 & \multicolumn{3}{|c|}{3 alpha } \\
\hline 2 & 7 & 6 & 9 & 0 & $! 27$ & A14 & & $A(7,6,9)$ & H31 & $-C$ & $3-C 4$ & \multicolumn{3}{|l|}{$3 \mathrm{bl}$} \\
\hline 2 & 8 & 6 & 9 & 0 & $! 28$ & A15 & & $A(8,6,9)$ & H32 & $-C$ & $3-C 4$ & \multicolumn{3}{|l|}{$3 \mathrm{~b} 2$} \\
\hline 2 & 6 & 9 & 10 & 0 & $! 29$ & A16 & & $A(6,9,10)$ & C3 & $-\mathrm{C} 4$ & -04 & \multicolumn{3}{|l|}{$\begin{array}{l}4 \mathrm{~b} 1 \\
\end{array}$} \\
\hline 2 & 6 & 9 & 11 & 0 & $! 30$ & A17 & & $A(6,9,11)$ & C3 & - C4 & $-\mathrm{C} 5$ & \multirow{2}{*}{\multicolumn{3}{|c|}{$\begin{array}{l}\text { r1a2 } \\
4 \mathrm{~b} 2\end{array}$}} \\
\hline 21 & 10 & 9 & 11 & 0 & ! 31 & A18 & & $A(10,9,11)$ & 04 & $-\mathrm{C} 4$ & $-\mathrm{C} 5$ & & & \\
\hline 2 & 11 & 11 & 9 & 0 & ! 32 & A19 & & $A(1,11,9)$ & $\mathrm{C} 1$ & $-\mathrm{C} 5$ & $-C 4$ & \multicolumn{3}{|c|}{ rla3 gamma } \\
\hline 2 & 11 & 11 & 12 & 0 & !33 & A20 & & $A(1,11,12)$ & $\mathrm{C} 1$ & $-\mathrm{C} 5$ & - H51 & \multicolumn{3}{|c|}{$5 \mathrm{~b} 1$} \\
\hline 2 & 11 & 11 & 13 & 0 & ! 34 & A21 & & $A(1,11,13)$ & $\mathrm{C} 1$ & $-\mathrm{C} 5$ & $-\mathrm{H} 52$ & $5 b 2$ & & \\
\hline 2 & 97 & 11 & 12 & 0 & $! 35$ & A22 & & $A(9,11,12)$ & $\mathrm{C} 4$ & $-\mathrm{C} 5$ & - H51 & $5 \mathrm{~b} 3$ & & \\
\hline 2 & 9 & 11 & 13 & 0 & !36 & A23 & & $A(9,11,13)$ & $\mathrm{C} 4$ & $-\mathrm{C} 5$ & - H52 & $5 b 4$ & & \\
\hline 21 & 12 & 11 & 13 & 0 & $! 37$ & A2 4 & & $A(12,11,13)$ & H51 & $-C$ & $5-$ H52 & 5 alpha & & \\
\hline 3 & 1 & 3 & 11 & 2 & $! 38$ & I1 & & $I(1,3,11,2)$ & $\mathrm{C} 1$ & $-\mathrm{C} 2-$ & C5-O1 & $\mathrm{C} 1$ & & \\
\hline 3 & 97 & 11 & 6 & 10 & $! 39$ & I1 & & $I(9,11,6,10)$ & & $-\mathrm{C} 1-$ & $\mathrm{C} 2-\mathrm{O} 4$ & $\mathrm{C} 4$ & & \\
\hline 41 & 11 & 1 & 3 & 6 & $! 40$ & D1 & & $D(11,1,3,6)$ & & $-\mathrm{C} 1-$ & $\mathrm{C} 2-\mathrm{C} 3$ & r1t 4 & & \\
\hline 4 & 1 & 3 & 6 & 9 & ! 41 & D2 & & $D(1,3,6,9)$ & $\mathrm{C} 1$ & $-\mathrm{C} 2-$ & $\mathrm{C} 3-\mathrm{C} 4$ & ret5 & & \\
\hline 4 & 3 & 6 & 9 & 11 & $! 42$ & D3 & & $D(3,6,9,11)$ & & $-\mathrm{C} 3-$ & $\mathrm{C} 4-\mathrm{C} 5$ & rlti & & \\
\hline 4 & 6 & 9 & 11 & 1 & $! 43$ & D4 & & $D(6,9,11,1)$ & & $-\mathrm{C} 4-$ & $\mathrm{C} 5-\mathrm{C} 1$ & r1t2 & & \\
\hline 4 & 97 & 11 & 1 & 3 & $! 44$ & D5 & & $D(9,11,1,3)$ & & $-\mathrm{C} 5-$ & $\mathrm{C} 1-\mathrm{C} 2$ & ret3 & & \\
\hline UMAT & & & 0 & 1 & & 0 & & ! row & $N$ nor & rmal & ization & & & \\
\hline 1 & 1 & 1 & & & & 2 & 2 & 1. & 3 & 3 & 1 & & & \\
\hline 4 & 4 & 1 & & & & 4 & & 1. & & & & & & \\
\hline 5 & 4 & 1 & & & & 5 & 4 & -1 & & & & & & \\
\hline 6 & 6 & 1 & & & & & & & & & & & & \\
\hline 7 & 7 & 1 & & & & 7 & 8 & 1. & & & & & & \\
\hline 8 & 7 & 1 & & & & 8 & 8 & -1 & & & & & & \\
\hline 9 & 9 & 1 & & & & 10 & 10 & 1 & & & & & & \\
\hline 11 & 11 & 1 & . & & & & & & & & & & & \\
\hline 12 & 12 & 1 & & & & 12 & 13 & 1. & & & & & & \\
\hline 13 & 12 & 1 & . & & & 13 & 13 & -1 & & & & & & \\
\hline 14 & 19 & 1 & & & & 14 & 20 & 5. & & & & & & \\
\hline 15 & 17 & 1 & & & & 15 & 18 & -1. & 15 & 21 & 1. & 15 & 22 & -1 \\
\hline 16 & 17 & -1 & & & & 16 & 18 & -1 & 16 & 21 & 1. & 16 & 22 & 1. \\
\hline 17 & 17 & $7-1$ & & & & 17 & 18 & 1. & 17 & 21 & 1. & 17 & 22 & -1 \\
\hline 18 & 25 & $5 \quad 1$ & & & & 18 & 26 & 5. & & & & & & \\
\hline 19 & 23 & 1 & & & & 19 & 24 & -1. & 19 & 27 & 1. & 19 & 28 & -1 \\
\hline 20 & 23 & -1 & & & & 20 & 24 & -1 & 20 & 27 & 1. & 20 & 28 & 1. \\
\hline 21 & 23 & $3-1$ & & & & 21 & 24 & 1. & 21 & 27 & 1. & 21 & 28 & -1 \\
\hline 22 & 32 & 1 & & & & 22 & 37 & 5. & & & & & & \\
\hline 23 & 33 & 1 & & & & 23 & 34 & -1 & 23 & 35 & 1. & 23 & 36 & -1. \\
\hline 24 & 33 & 1 & & & & 24 & 34 & 1. & 24 & 35 & -1. & 24 & 36 & -1 \\
\hline 25 & 33 & 1 & & & & 25 & 34 & -1. & 25 & 35 & -1. & 25 & 36 & 1. \\
\hline 26 & 38 & 1 & & & & & & & & & & & & \\
\hline 27 & 15 & 1 & & & & 27 & 14 & -1 & & & & & & \\
\hline 28 & 39 & 1 & & & & & & & & & & & & \\
\hline 29 & 29 & 1 & & & & 29 & 31 & -1 & & & & & & \\
\hline 30 & 25 & 1 & & & & 30 & 30 & -0.81 & 30 & 32 & 0.31 & 30 & 16 & 0.31 \\
\hline 30 & 19 & -0 & .81 & & & & & & & & & & & \\
\hline
\end{tabular}




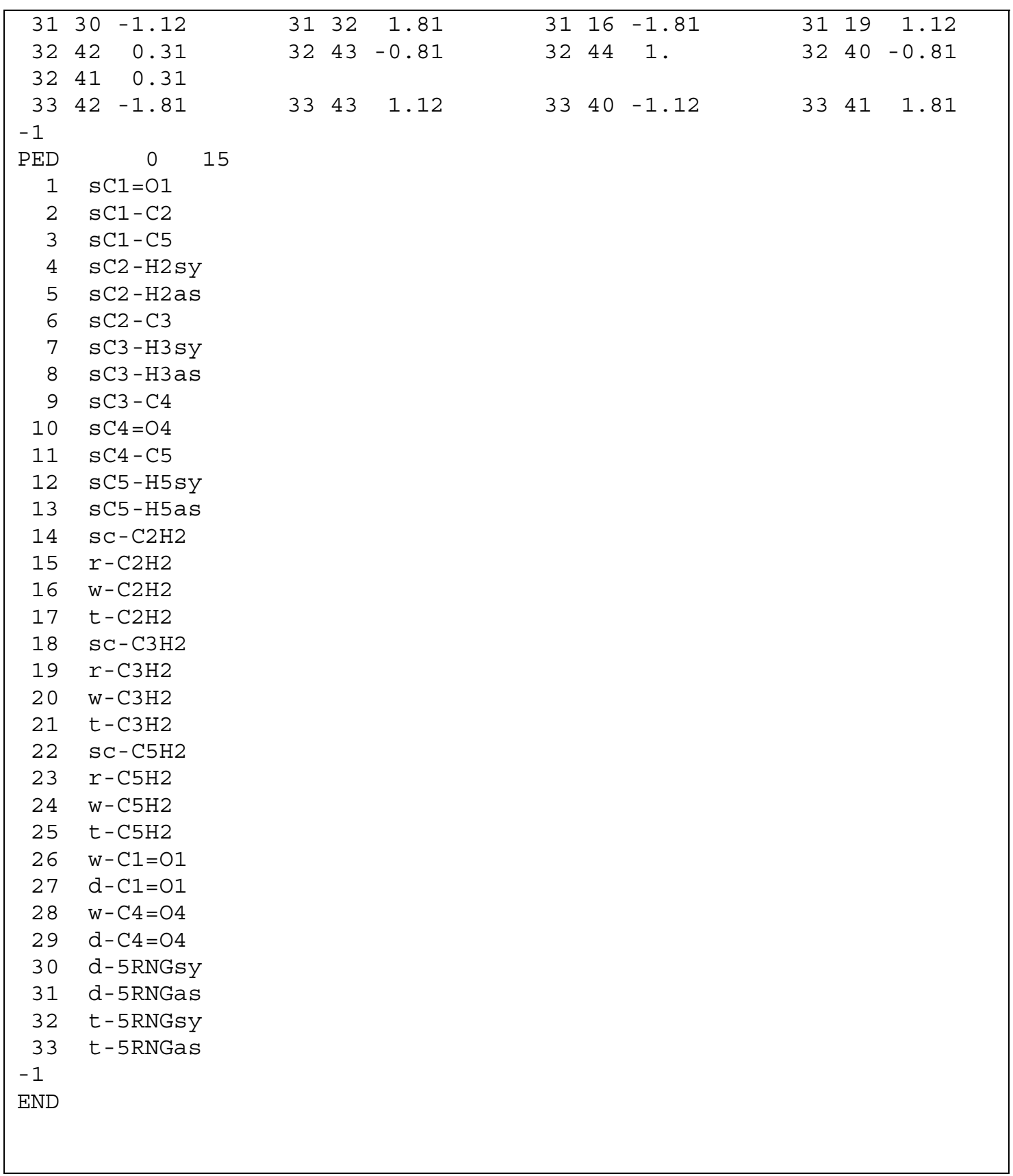

Table 35: Parameterization script (MM): Bond, angle and torsion parameter optimization for CPD

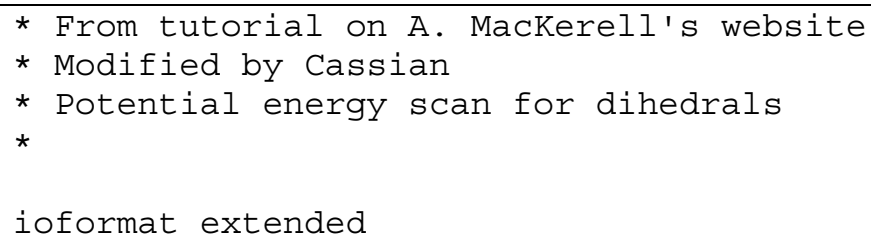




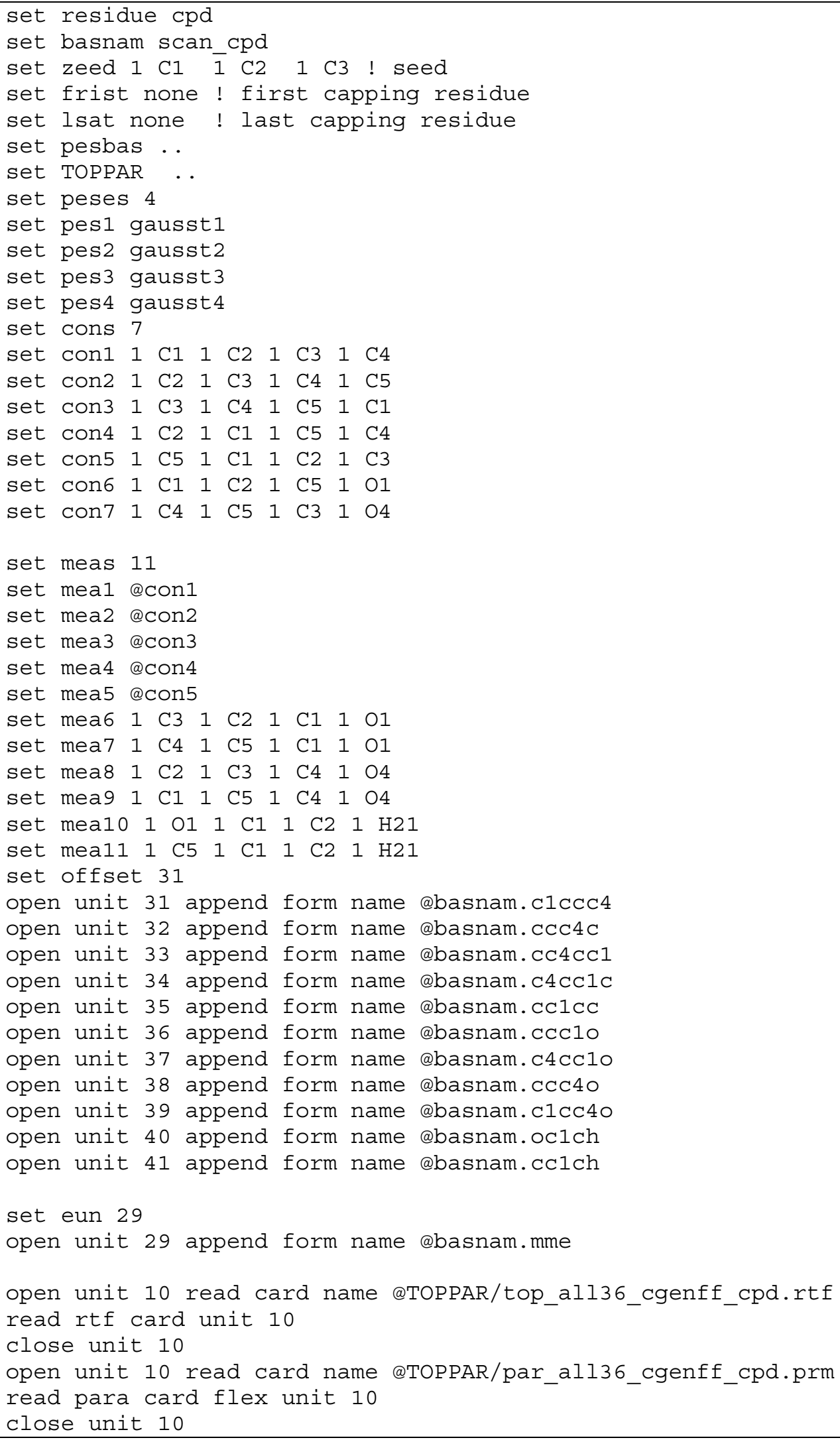




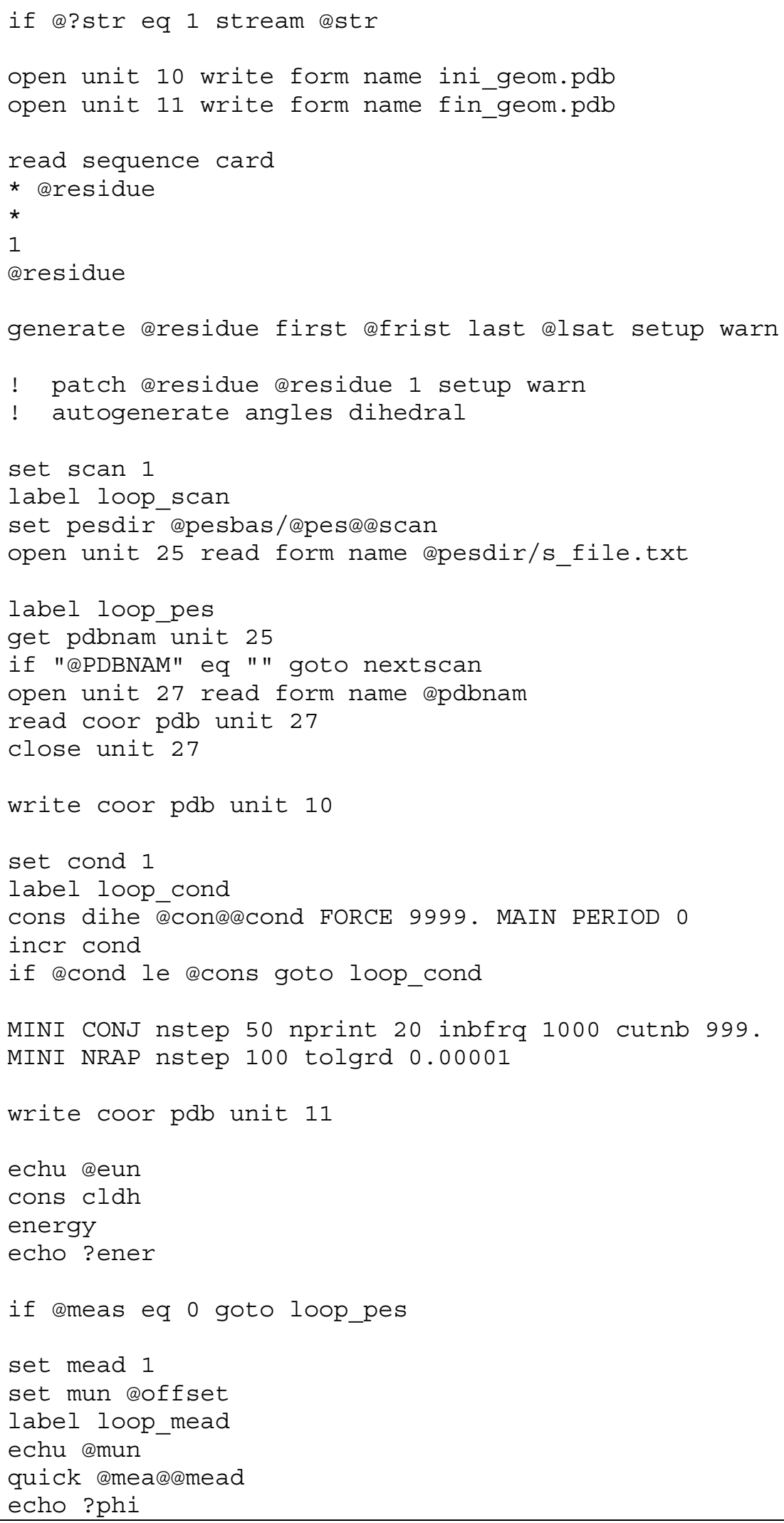




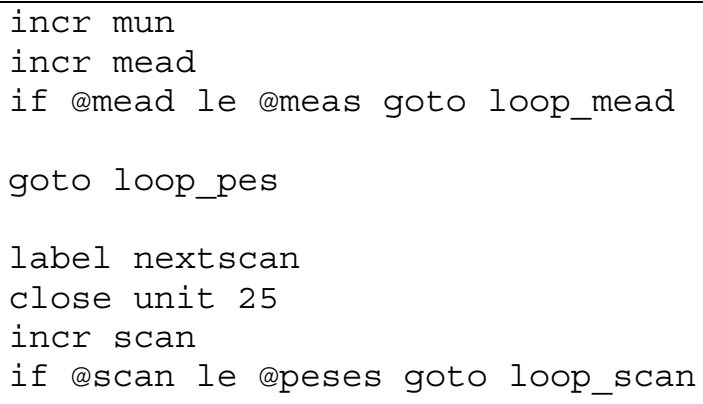

Table 36: Parameterization script (MM): Potential energy scans of dihedrals and impropers for CPD 


\section{A3 Scripts used for setup and run of molecular dynamic simulations}

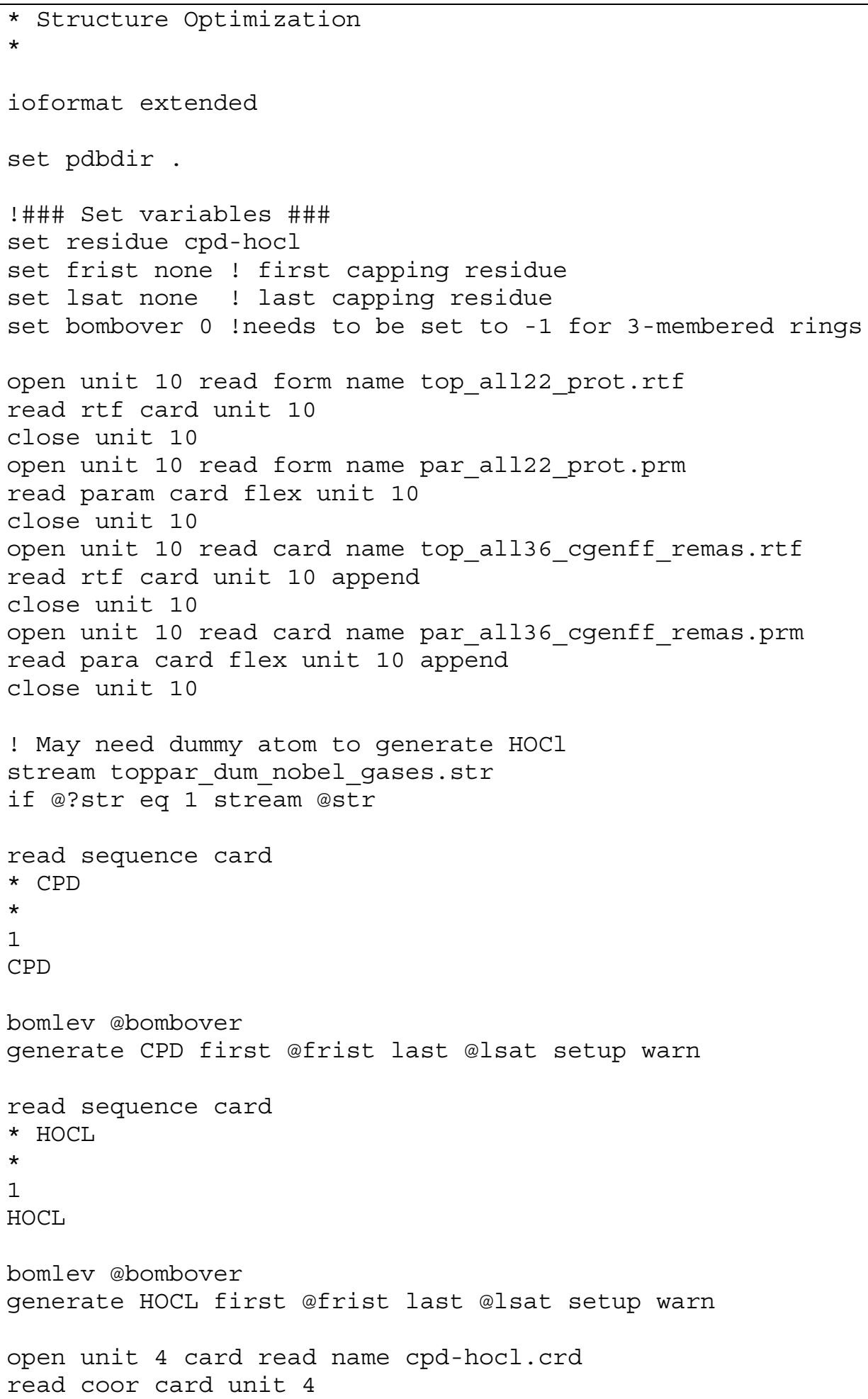


coor print

open unit 30 write form name @residue_init.pdb

write coor pdb unit 30

close unit 30

!minimize structure and calculate dipole moment

MINI CONJ nstep 200 nprint 20 inbfrq 1000 cutnb 999.

MINI NRAP nstep 50 tolgrd 0.00001

!save minimized geometry

coor print

open unit 30 write form name @residue_min.psf

write psf card unit 30

close unit 30

open unit 30 write form name @residue_min.crd

write coor card unit 30

open unit 30 write form name @residue_min.pdb

write coor pdb unit 30

close unit 30

stop

Table 37: Initial minimization script for CPD and HOCI

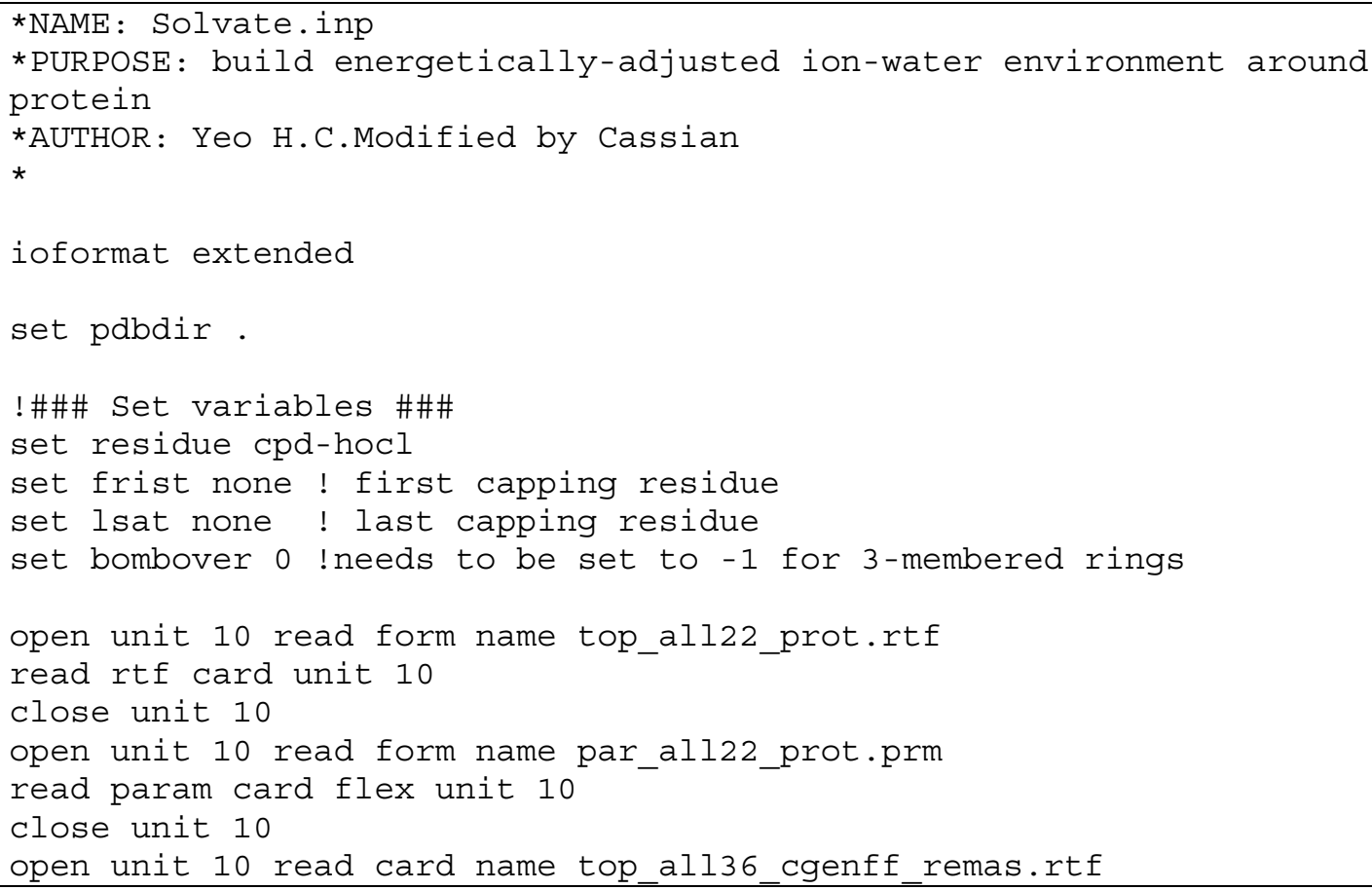




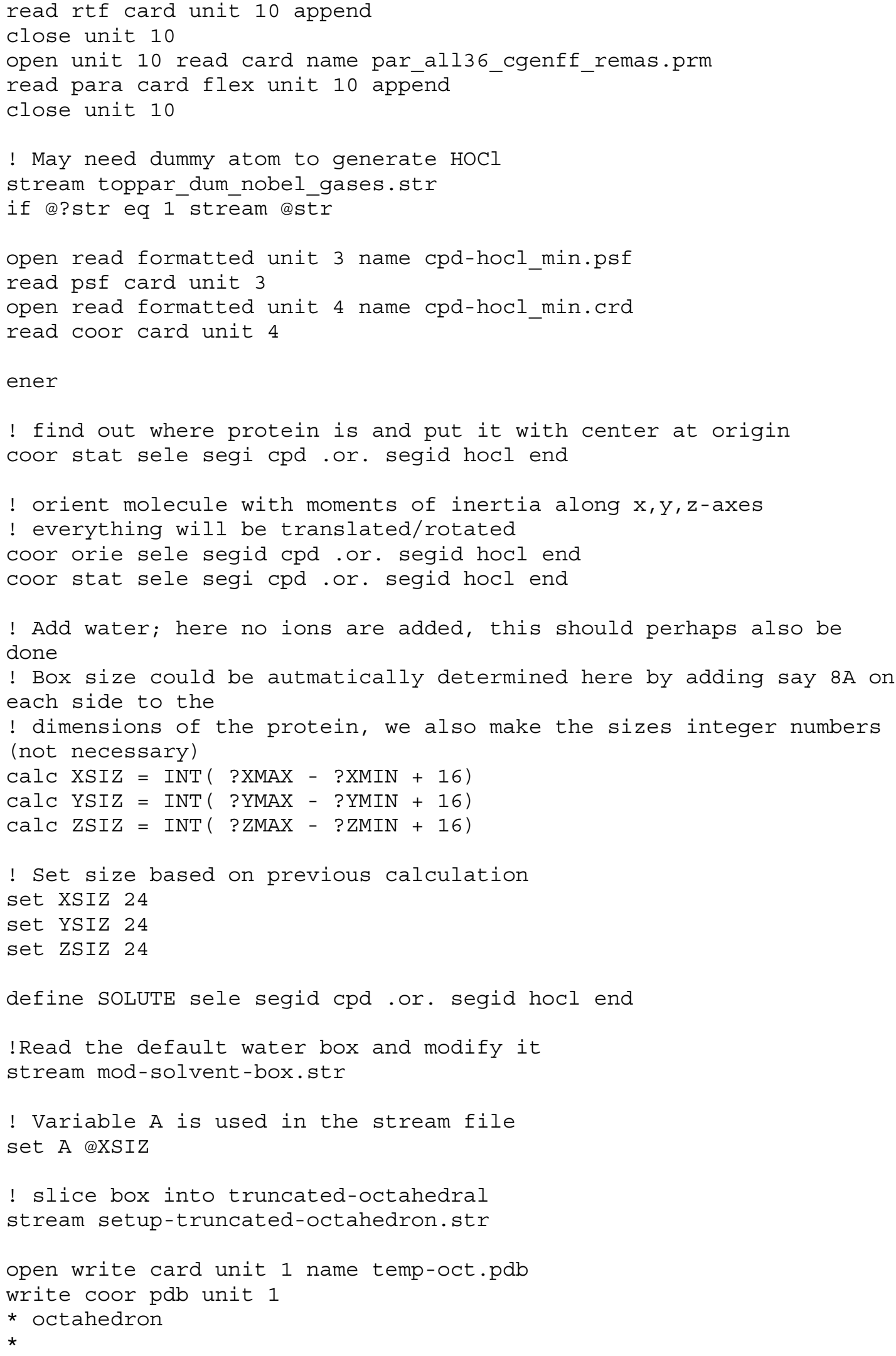




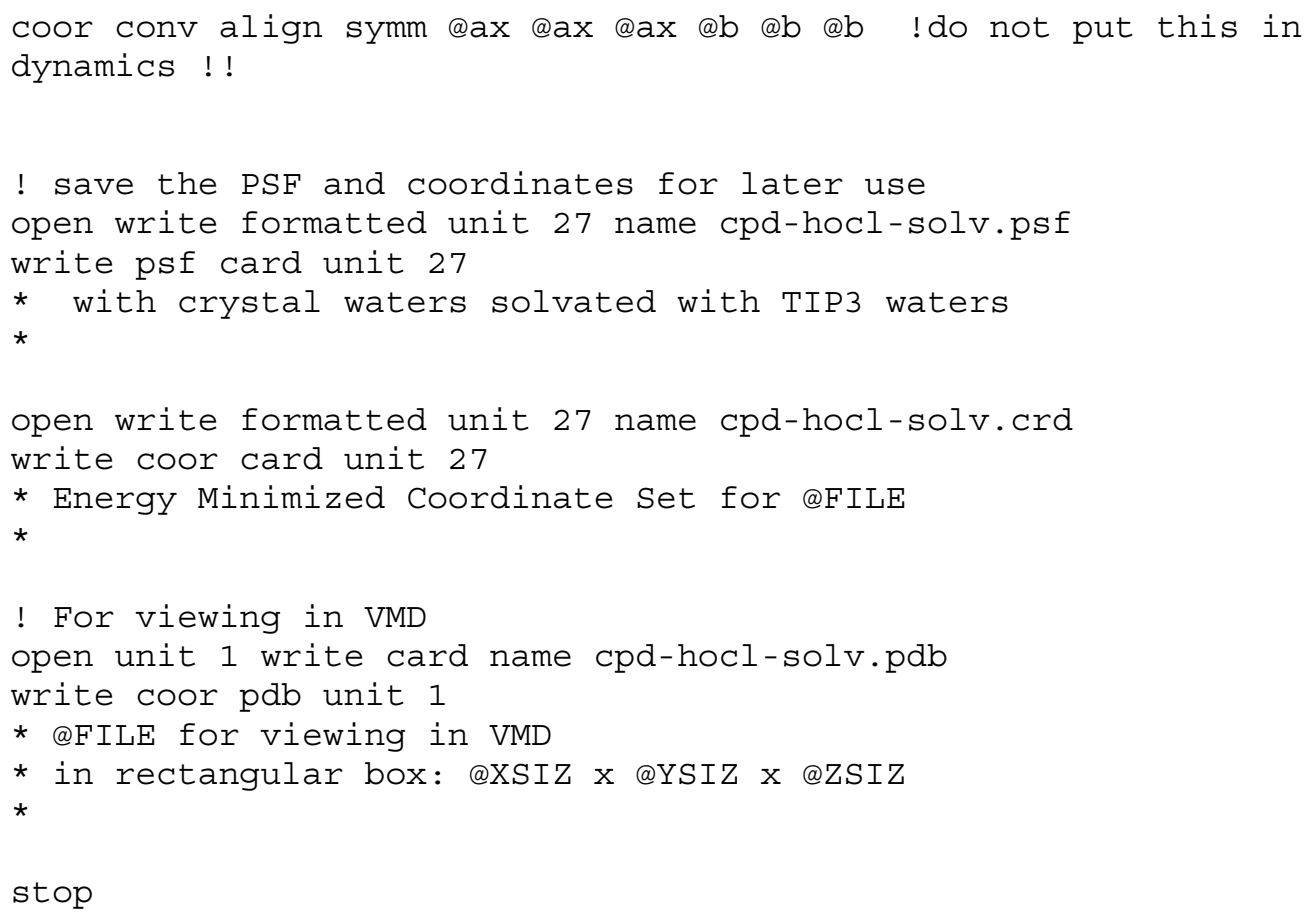

Table 38: Script for solvating CPD and $\mathrm{HOCl}$ in a truncated octahedron box

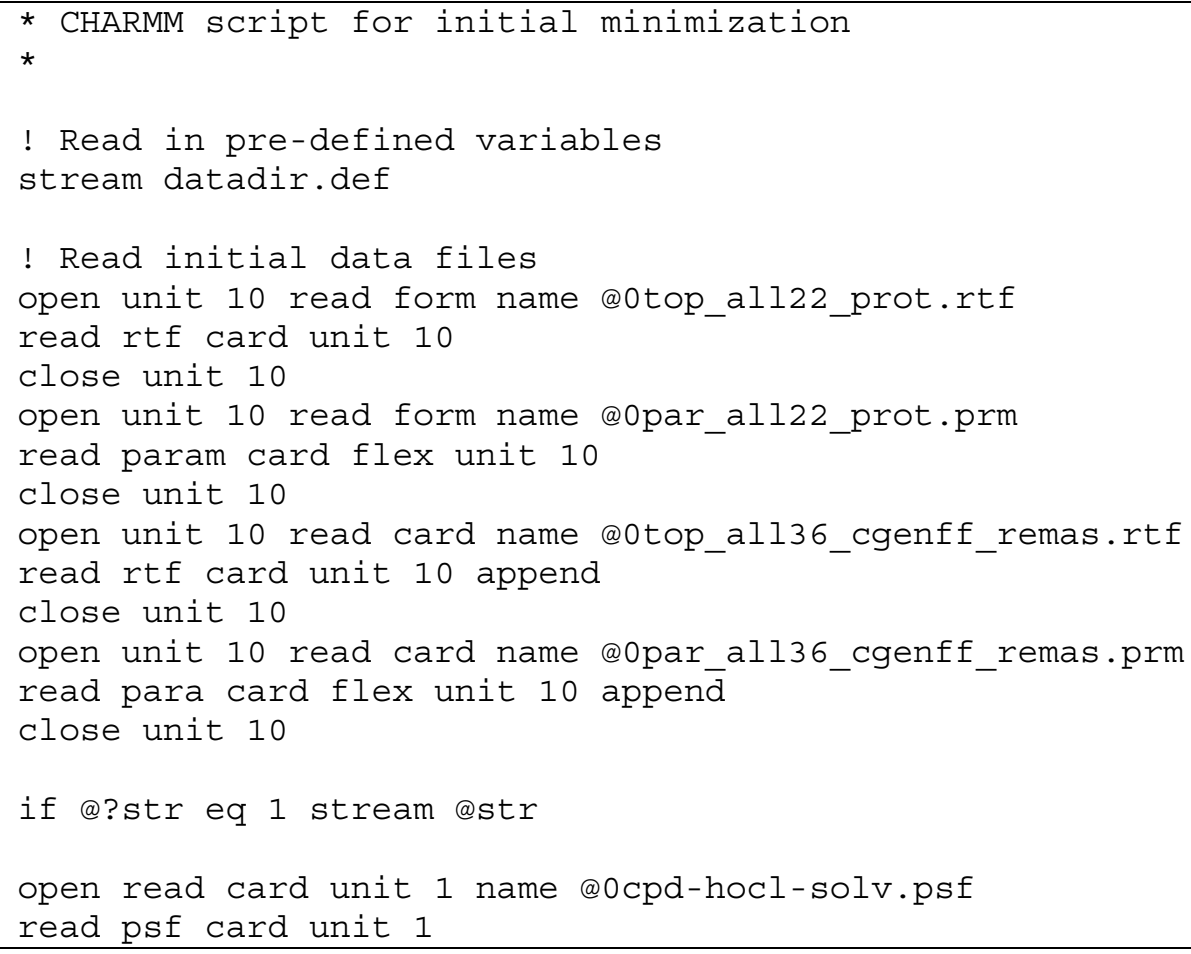




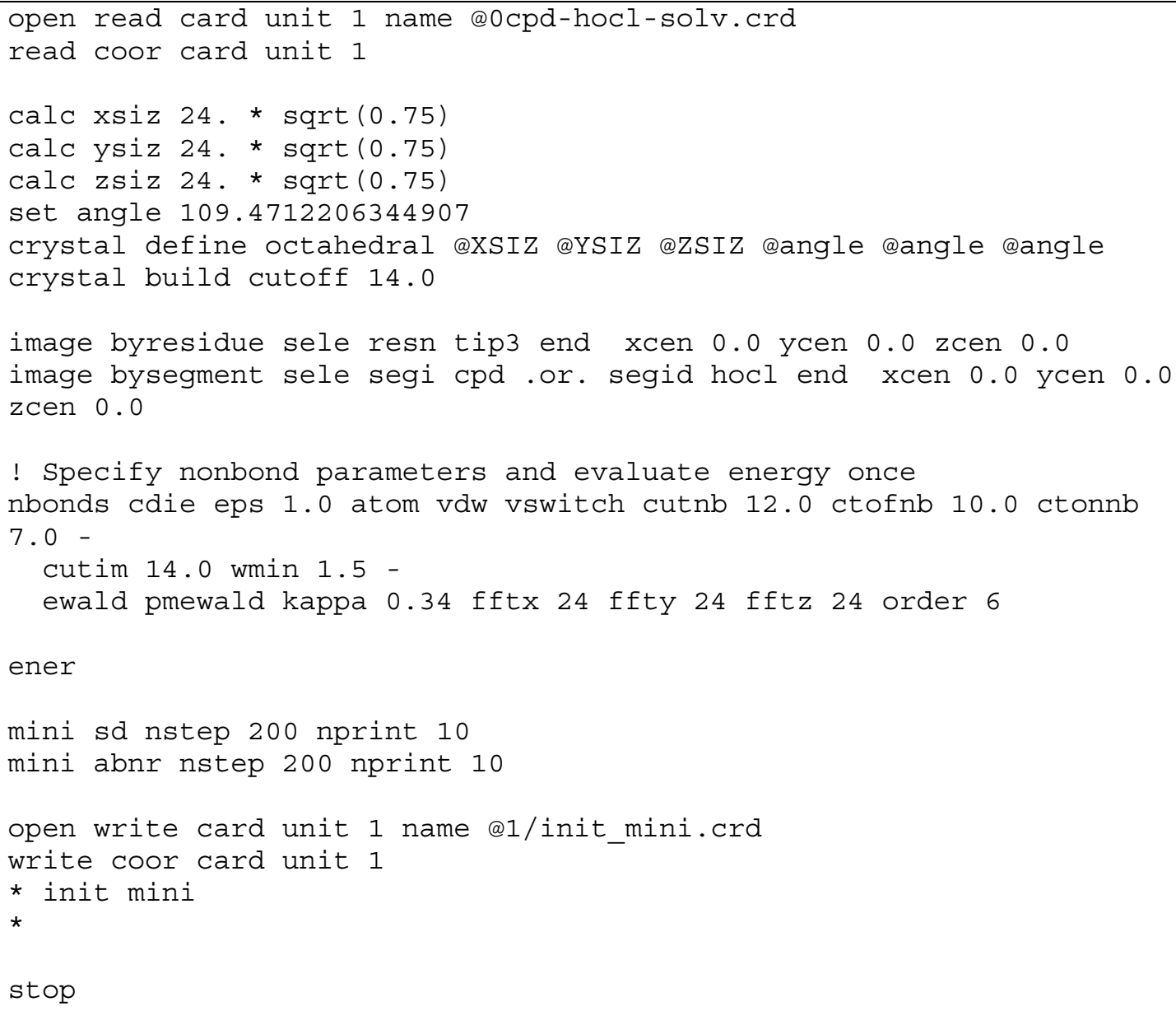

Table 39: Initial minimization script for solvated CPD and HOCI

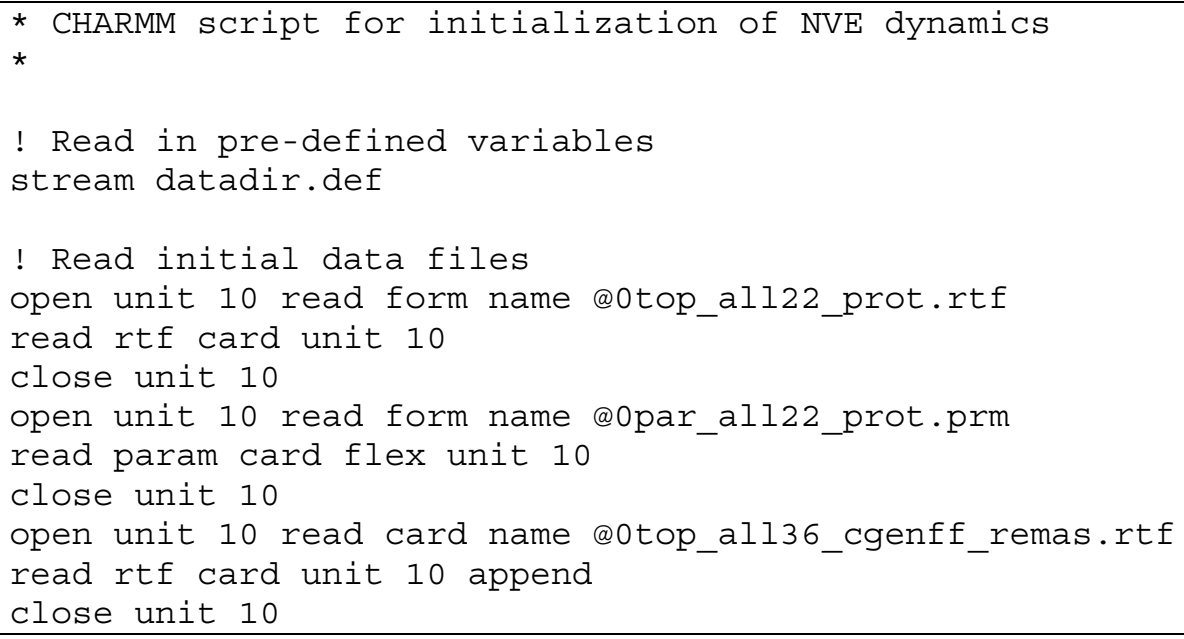




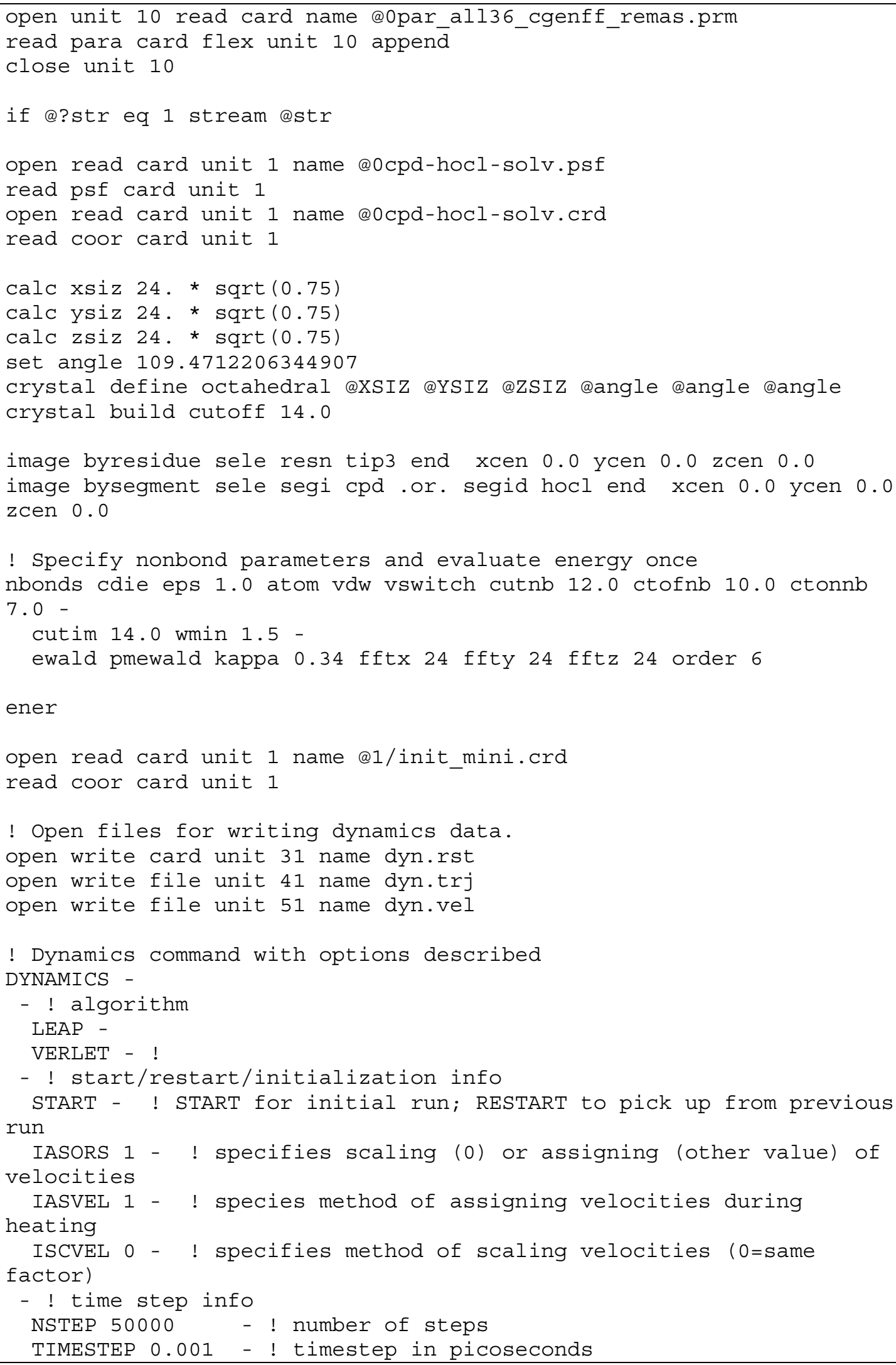




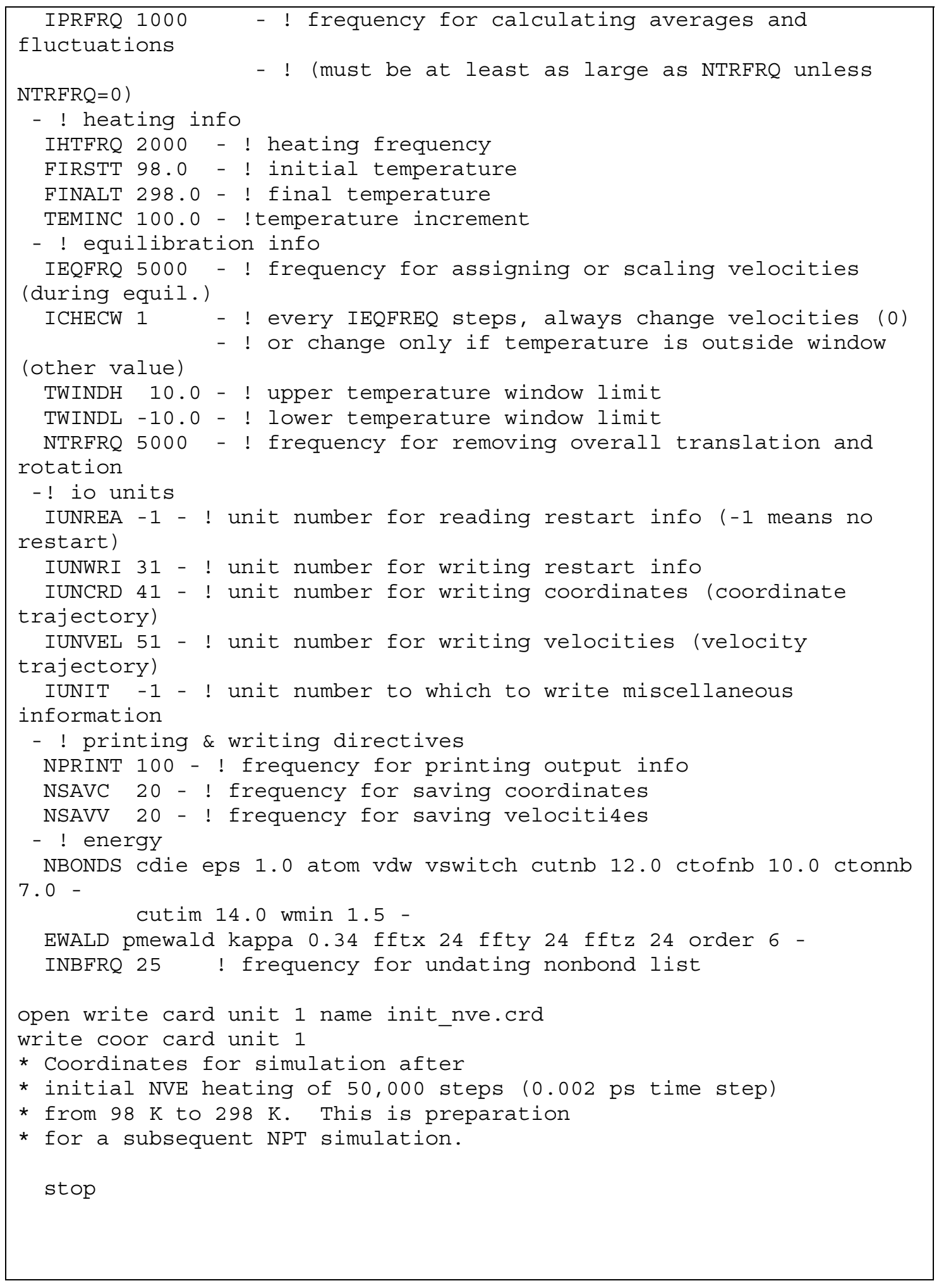

Table 40: Initial NVE dynamic simulation script (slowly heating the system to $298 \mathrm{~K}$ ) 


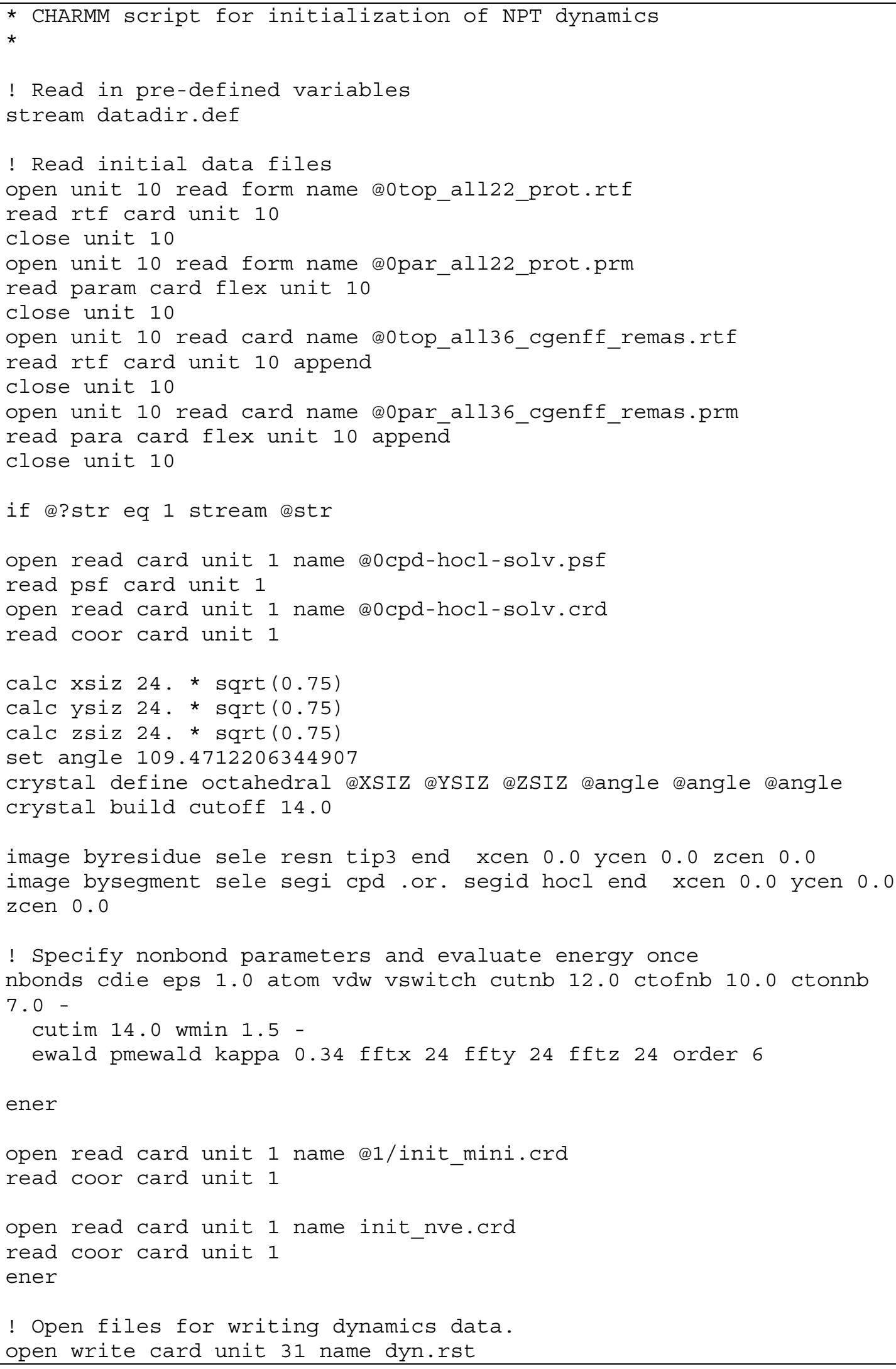




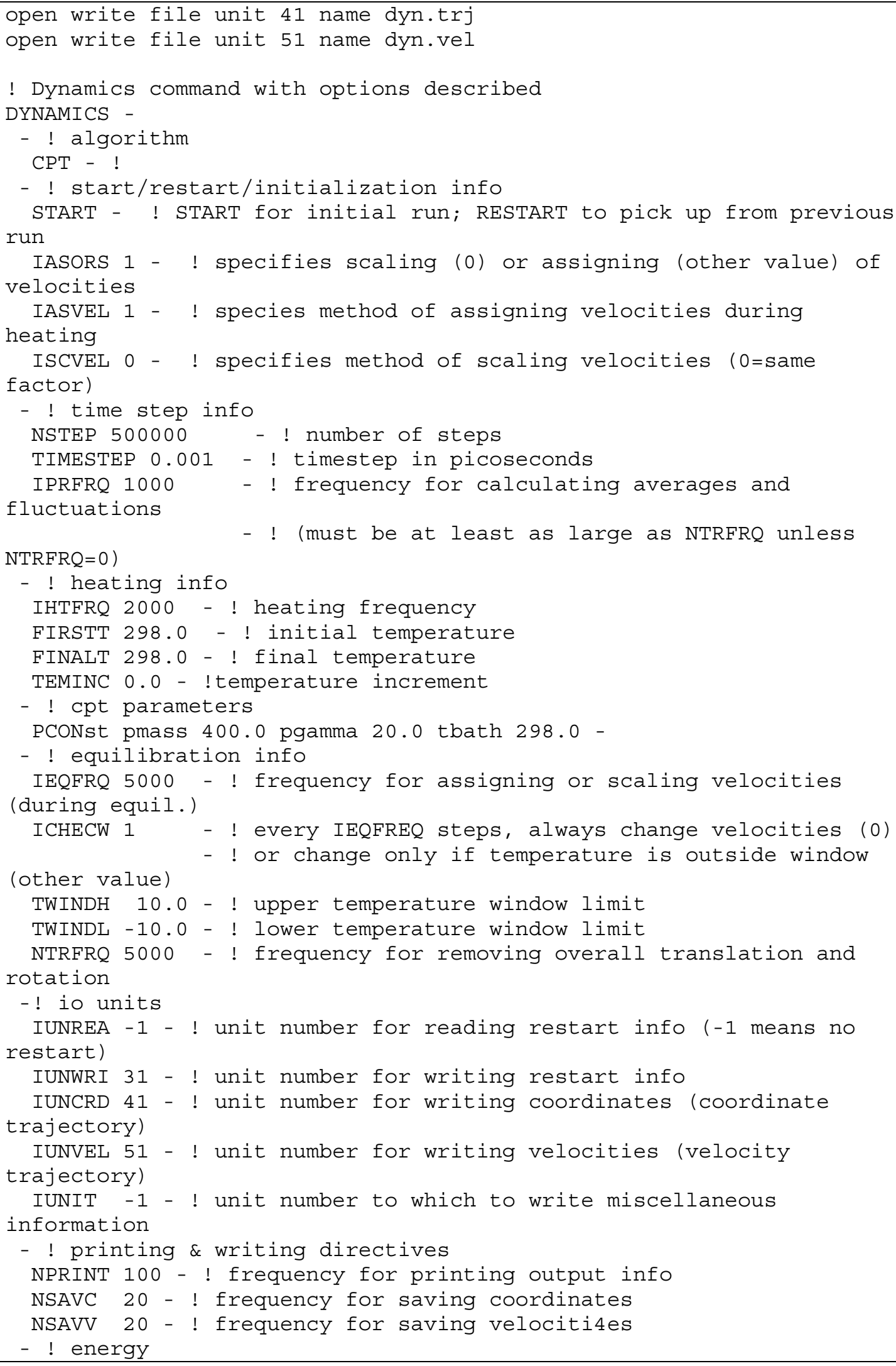




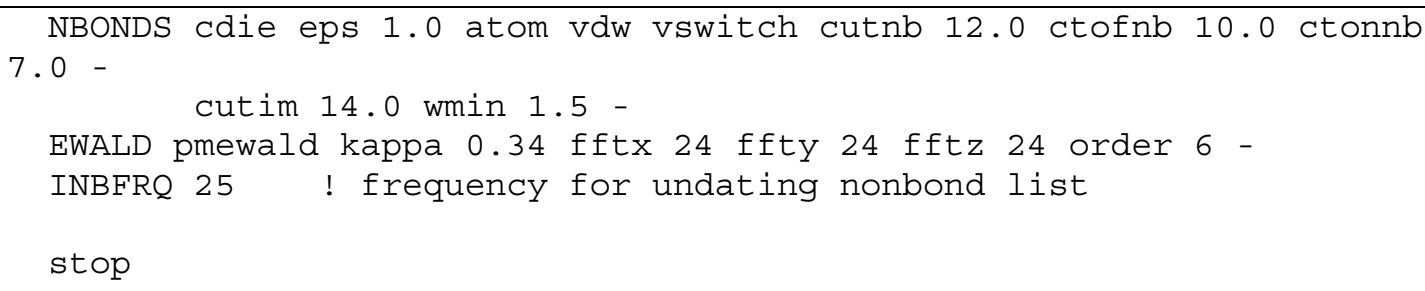

Table 41: Initial NPT dynamic simulation script

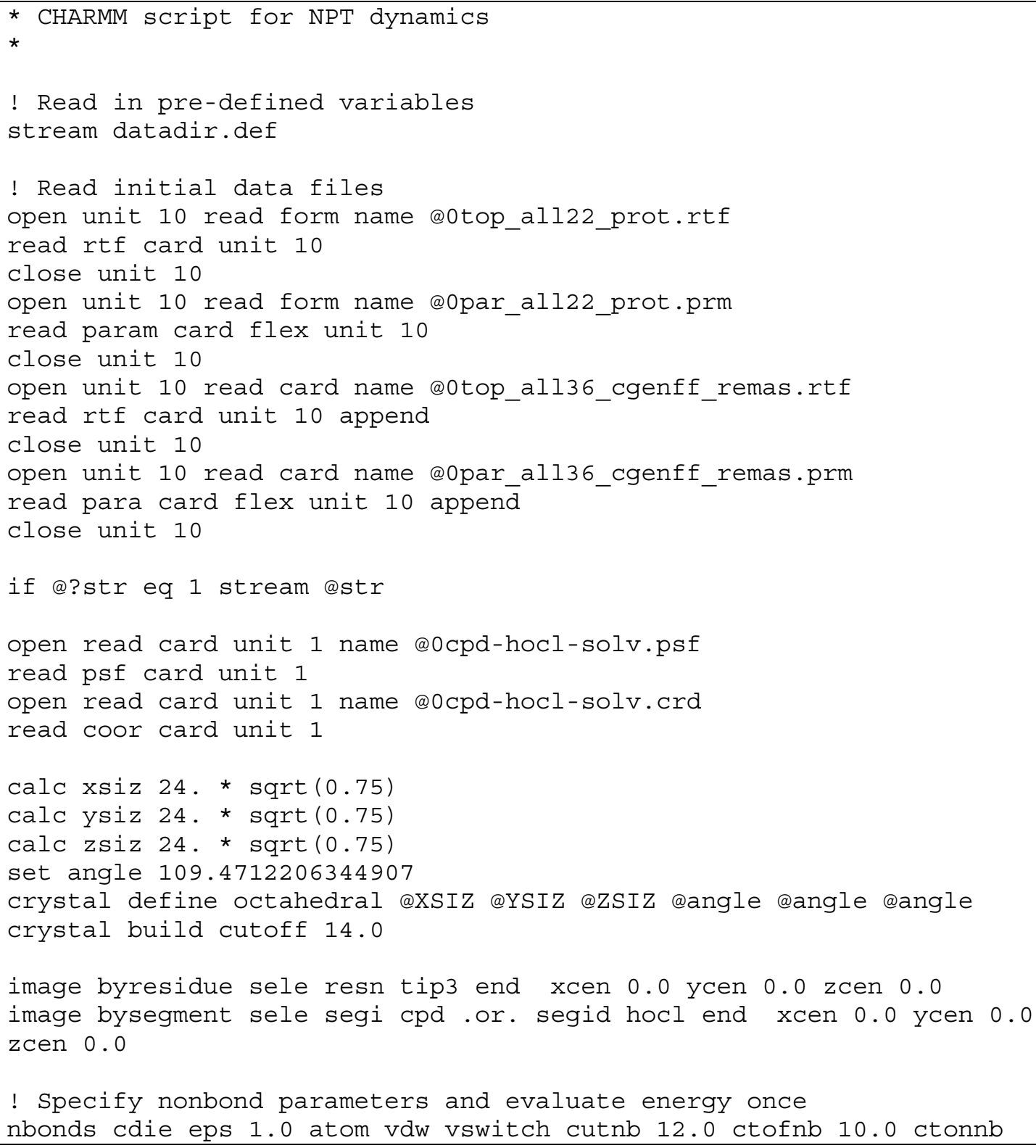




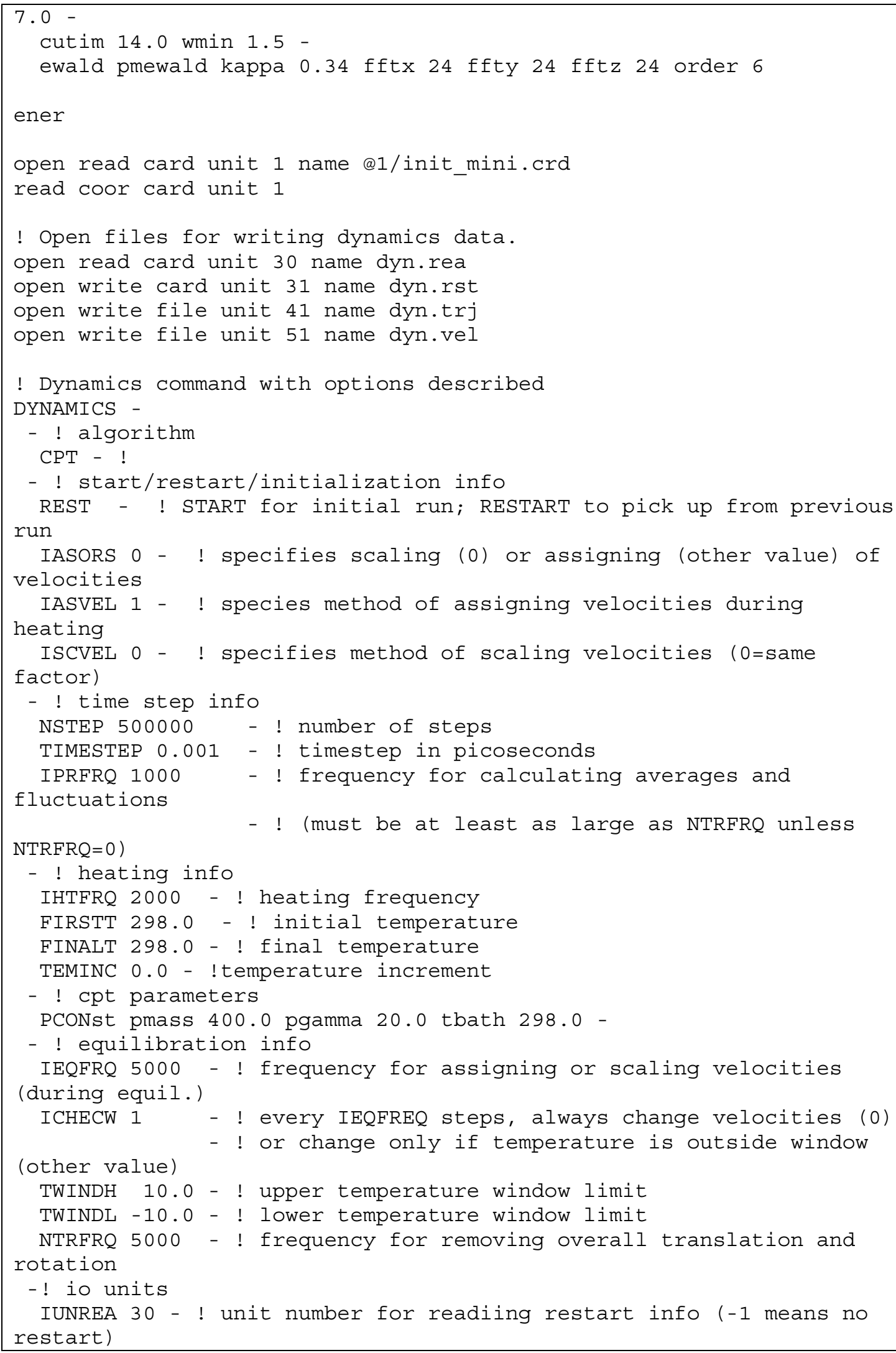




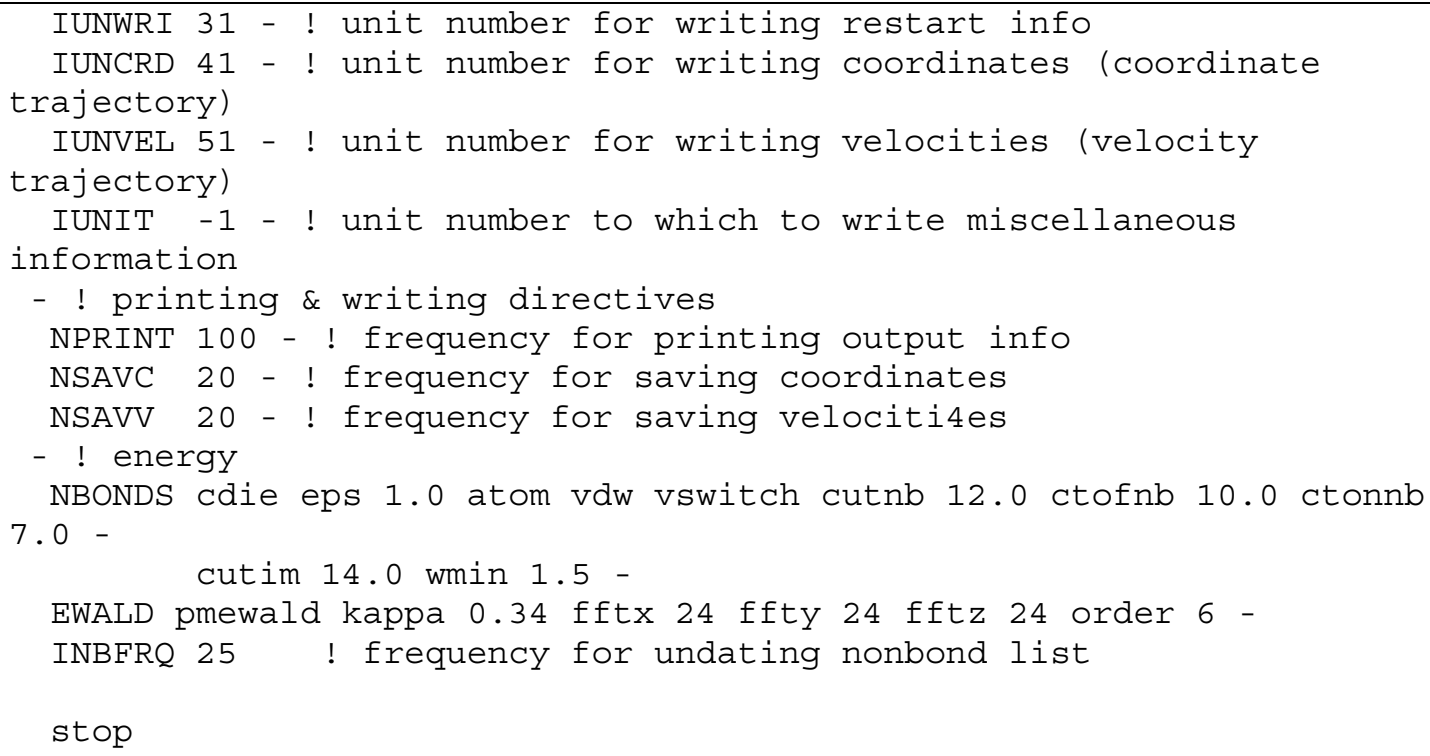

Table 42: Script for NPT dynamics simulation used for analysis

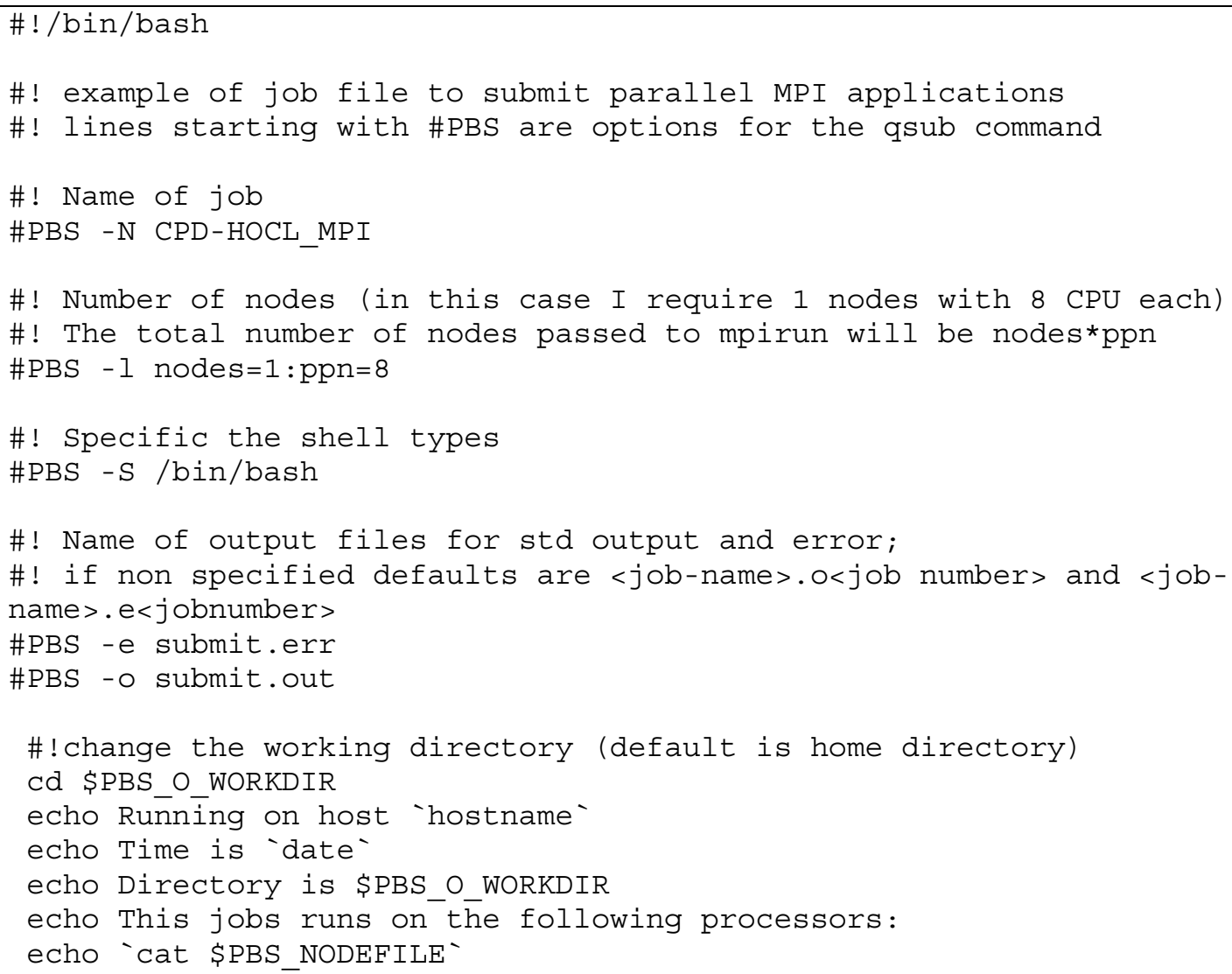




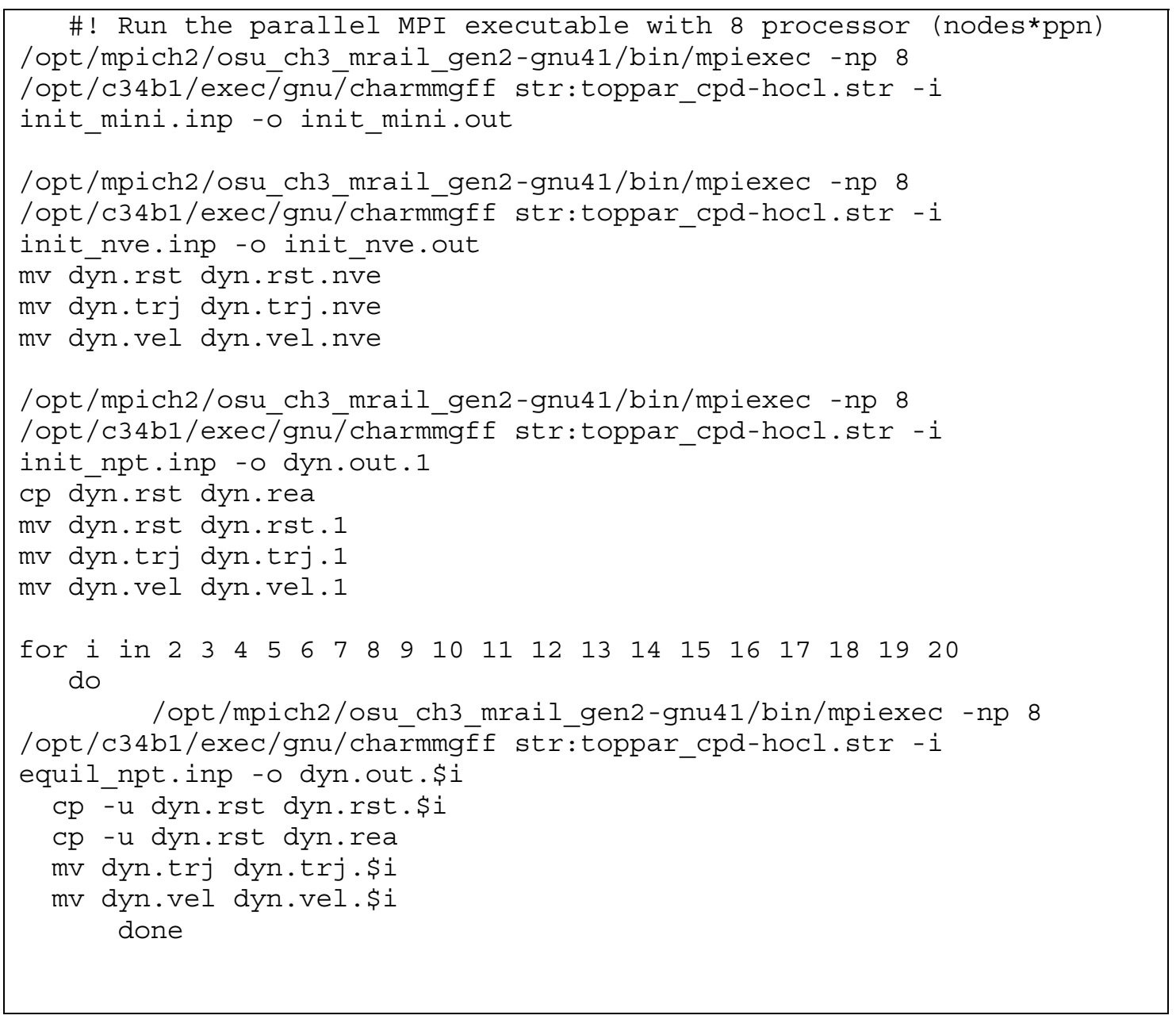

Table 43: Shell script to run various molecular dynamics scripts via queue on computer cluster and appropriately archive the output files

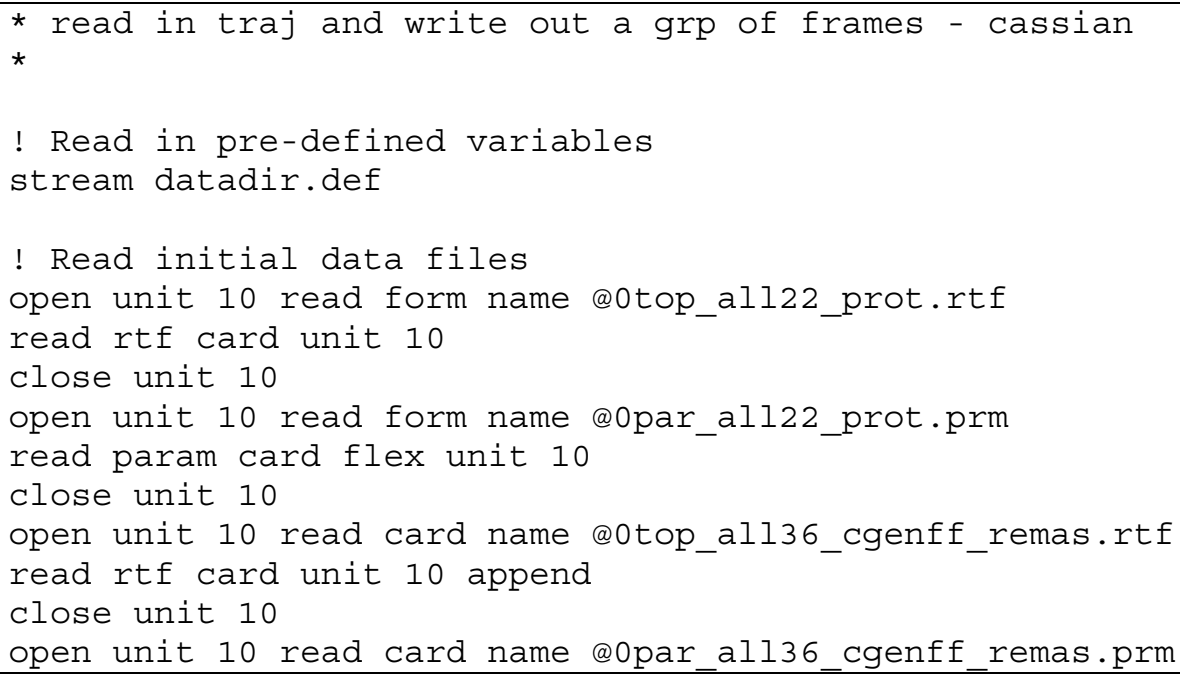




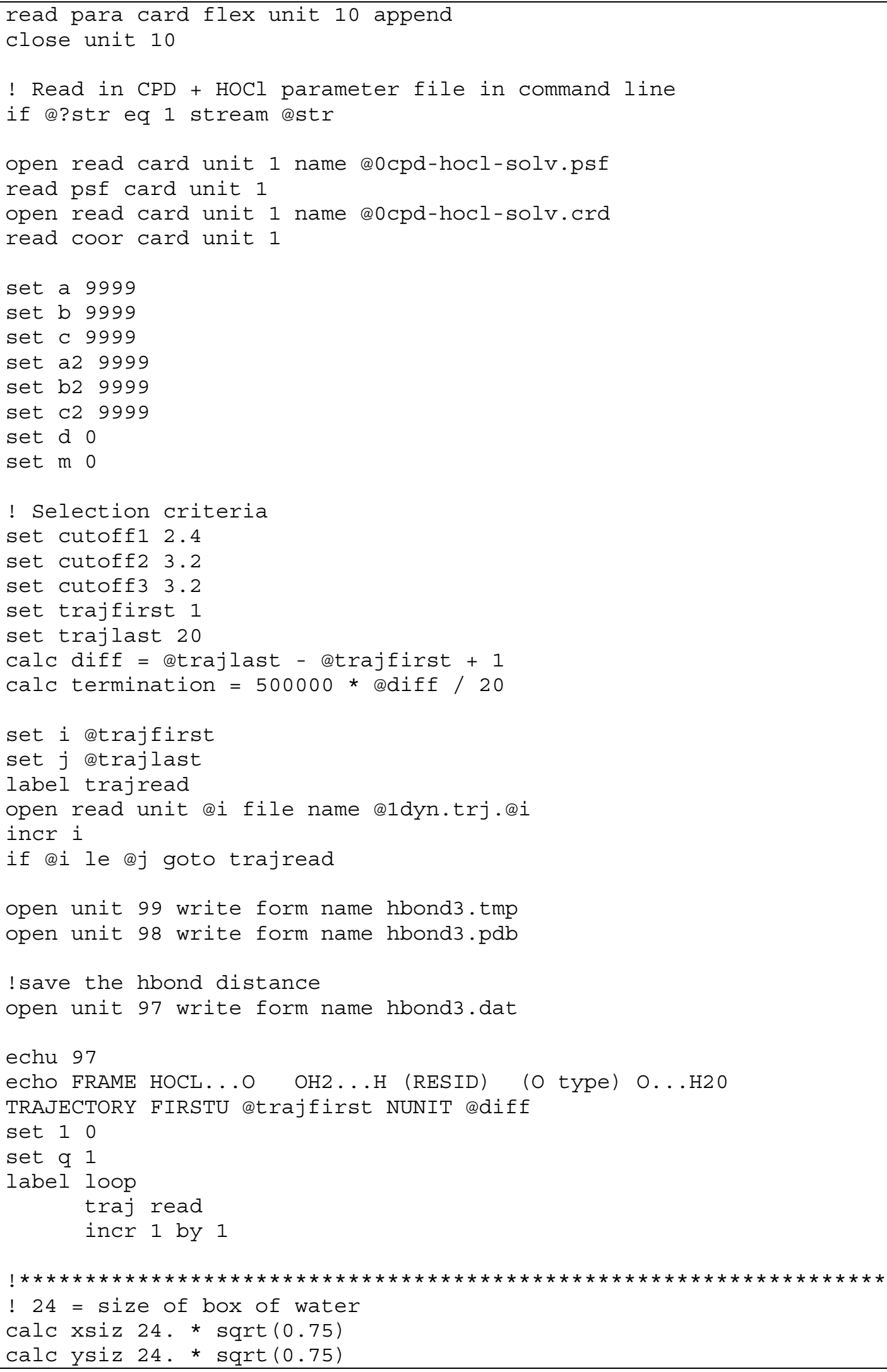




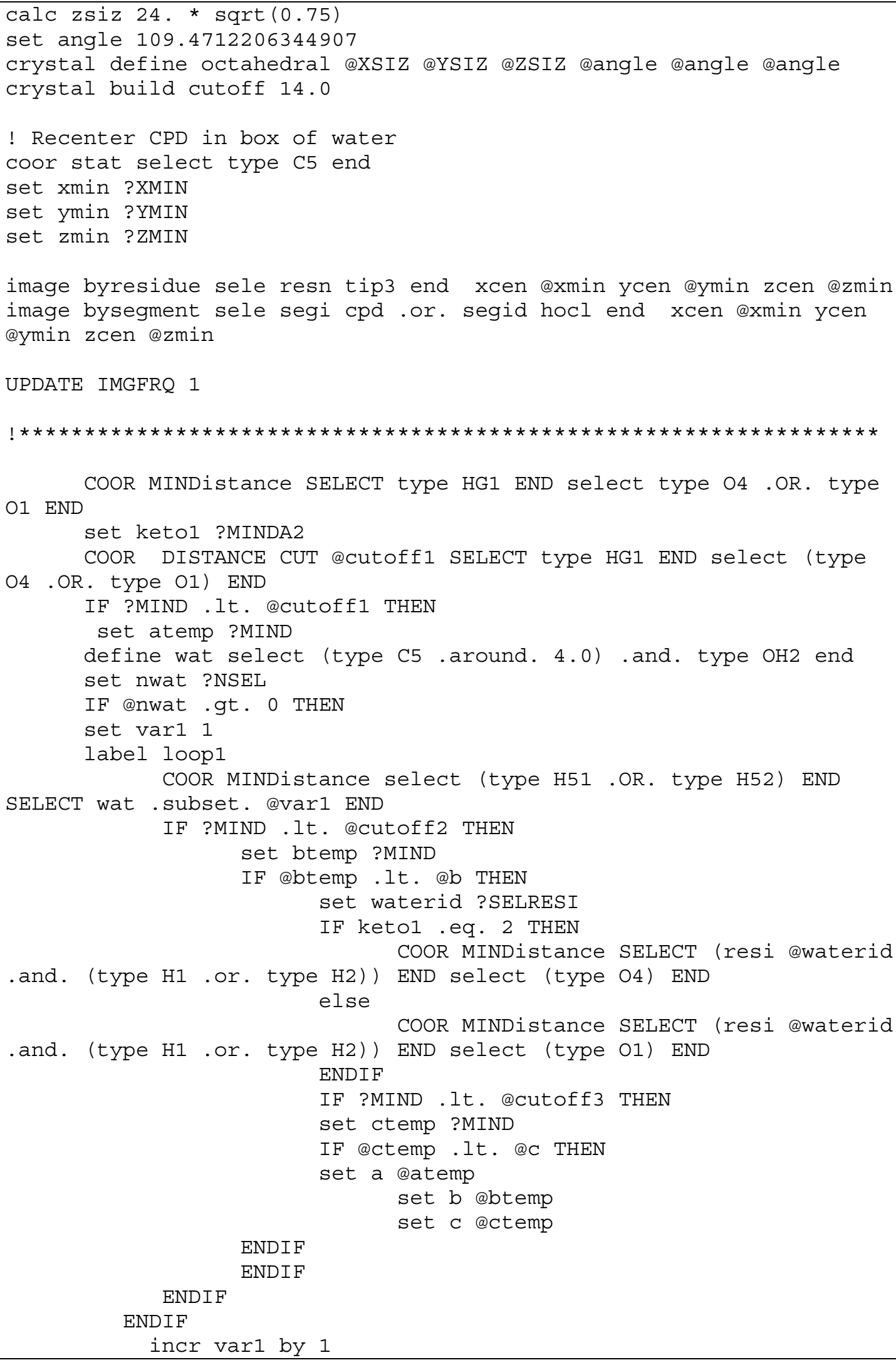




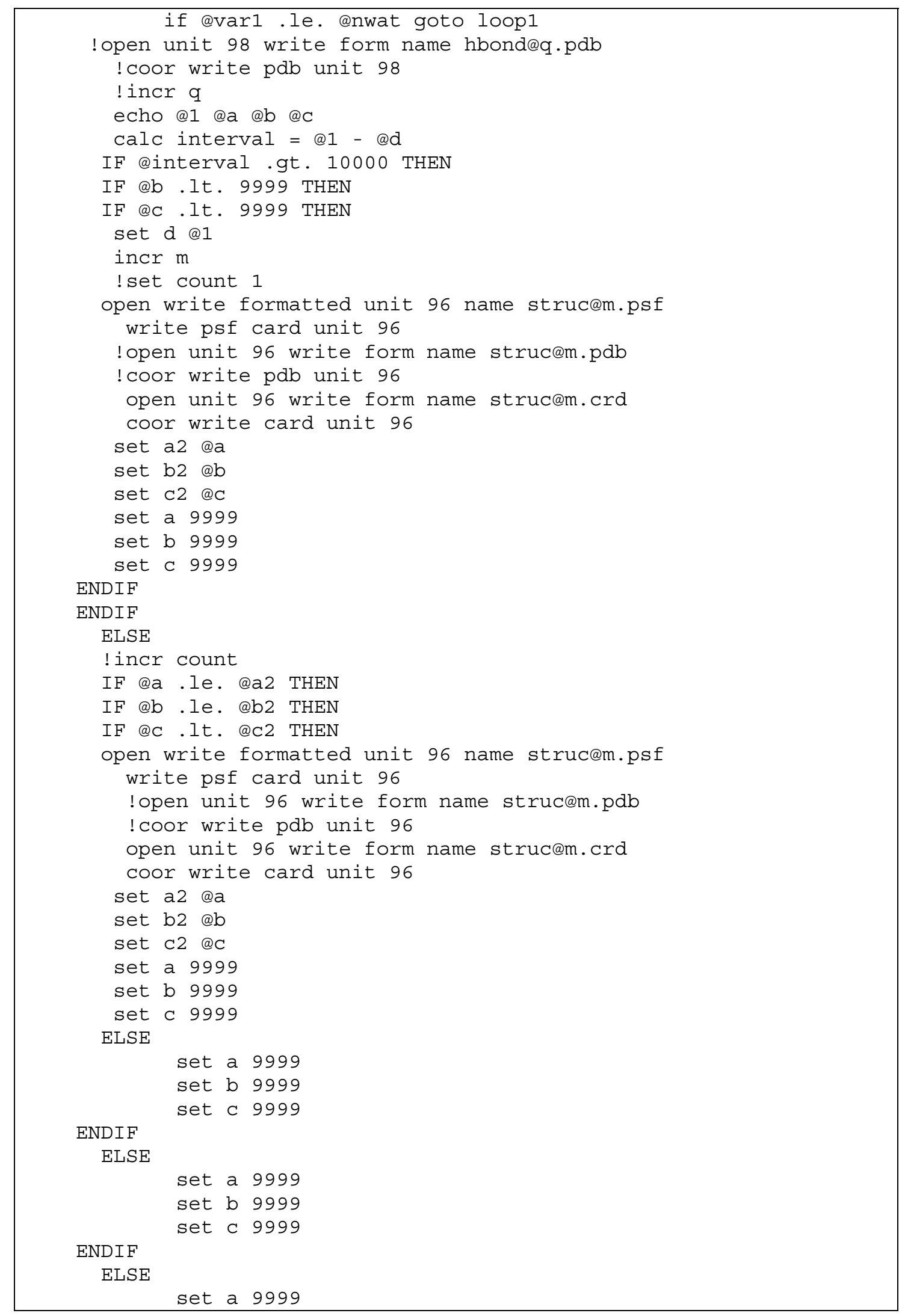




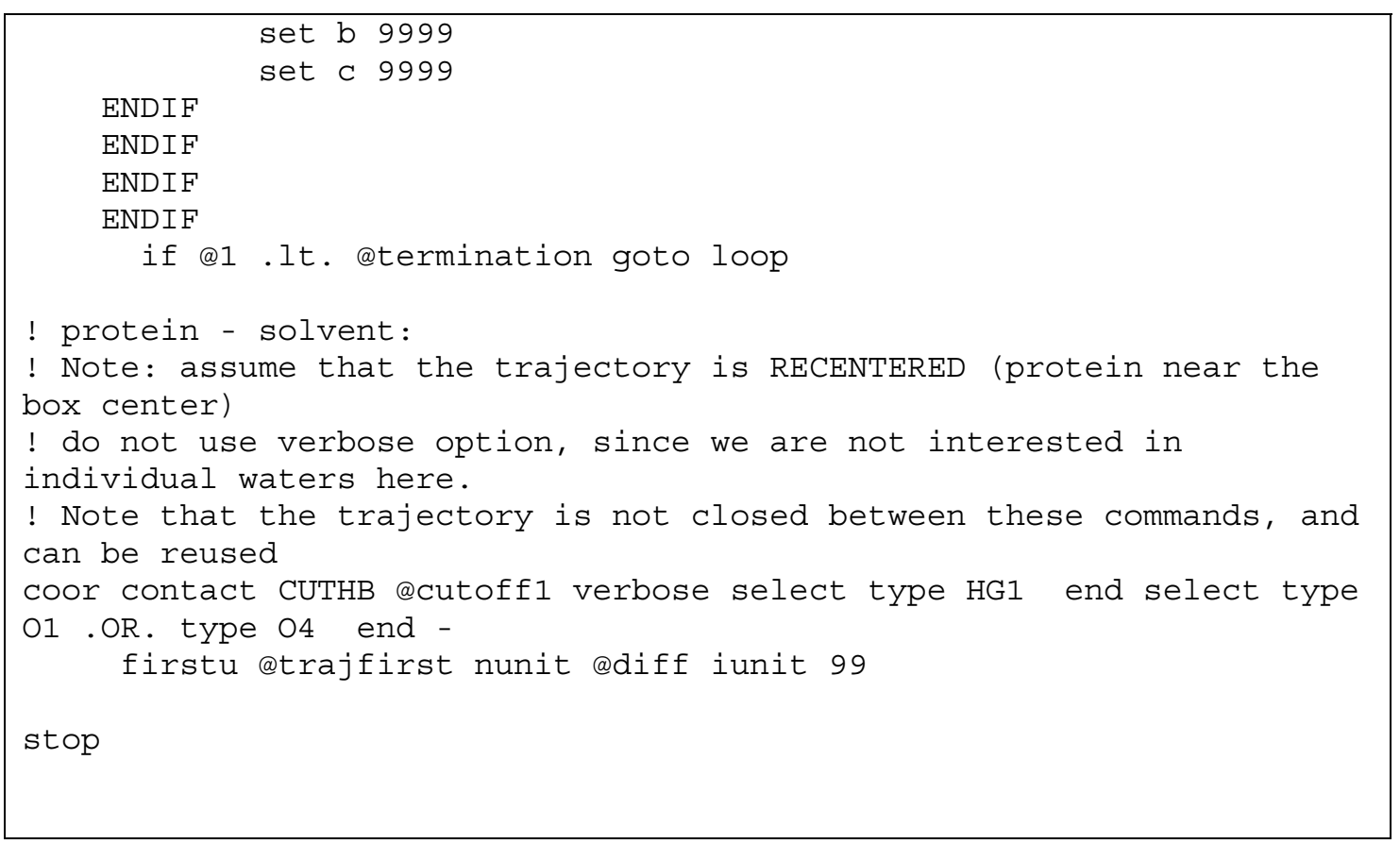

Table 44: Script for reading trajectory and saving coordinates of reactant precursors based on predetermined criteria 


\section{A4 Scripts used for calculation energy barrier in fully-solvated system.}

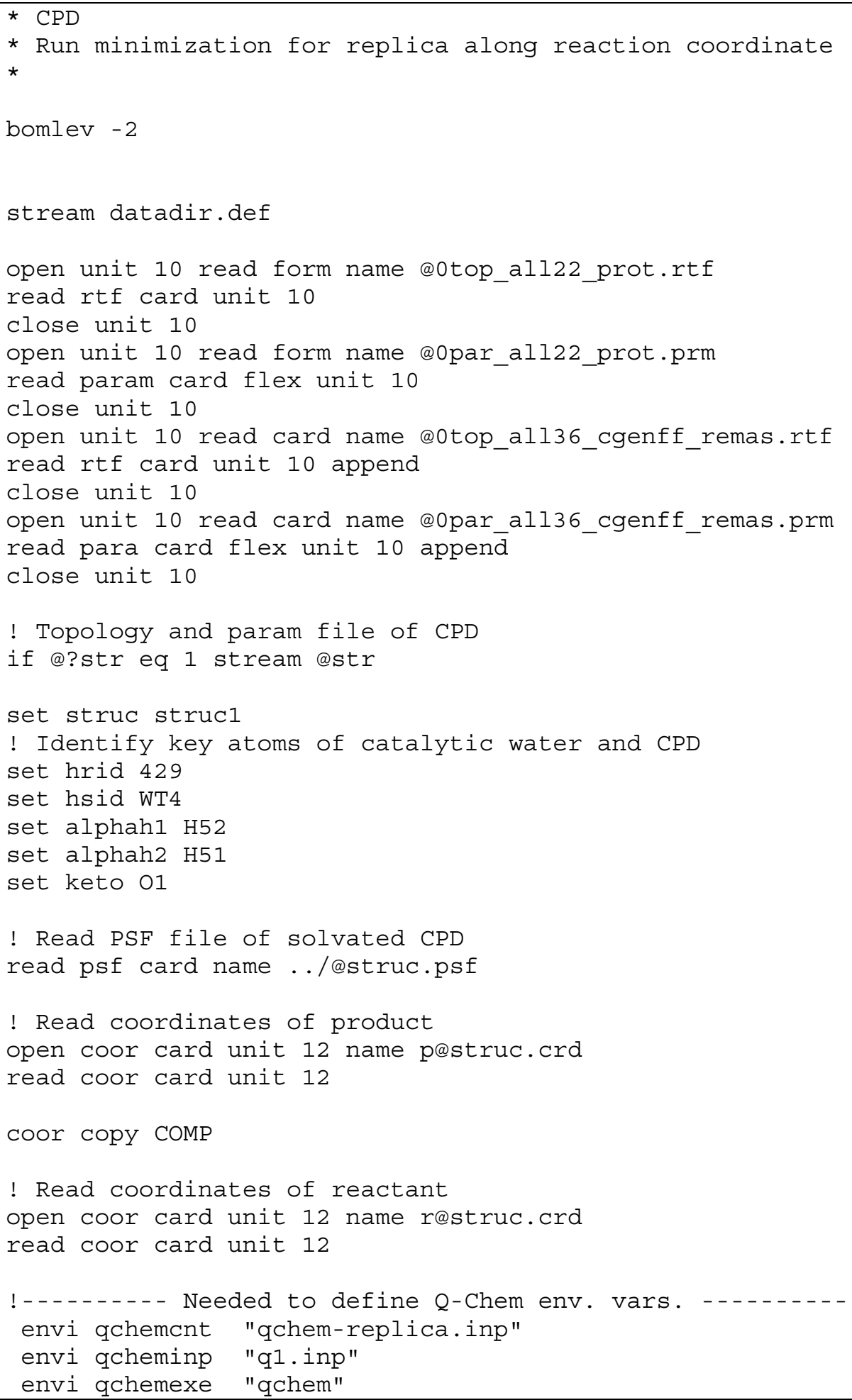




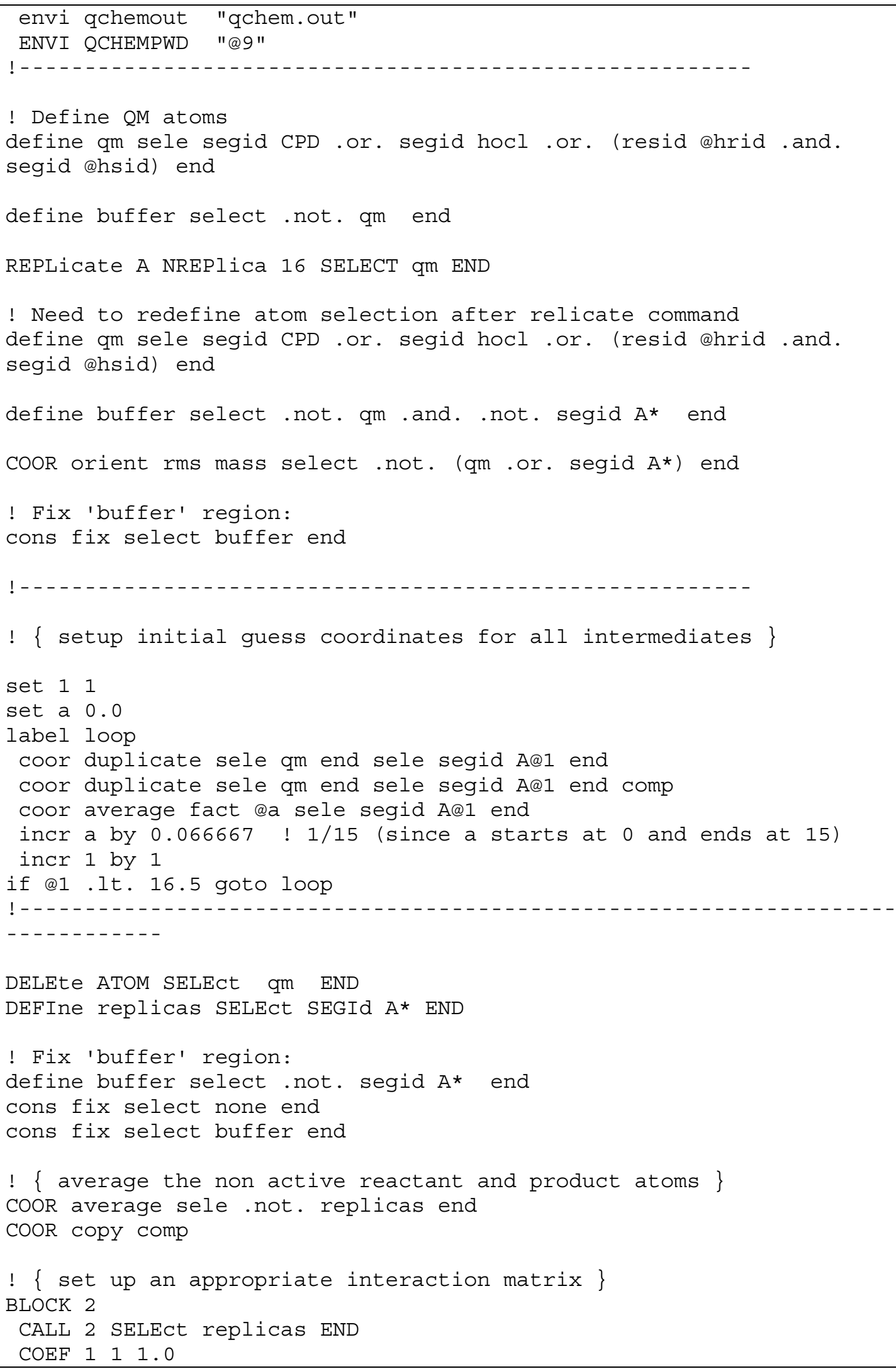




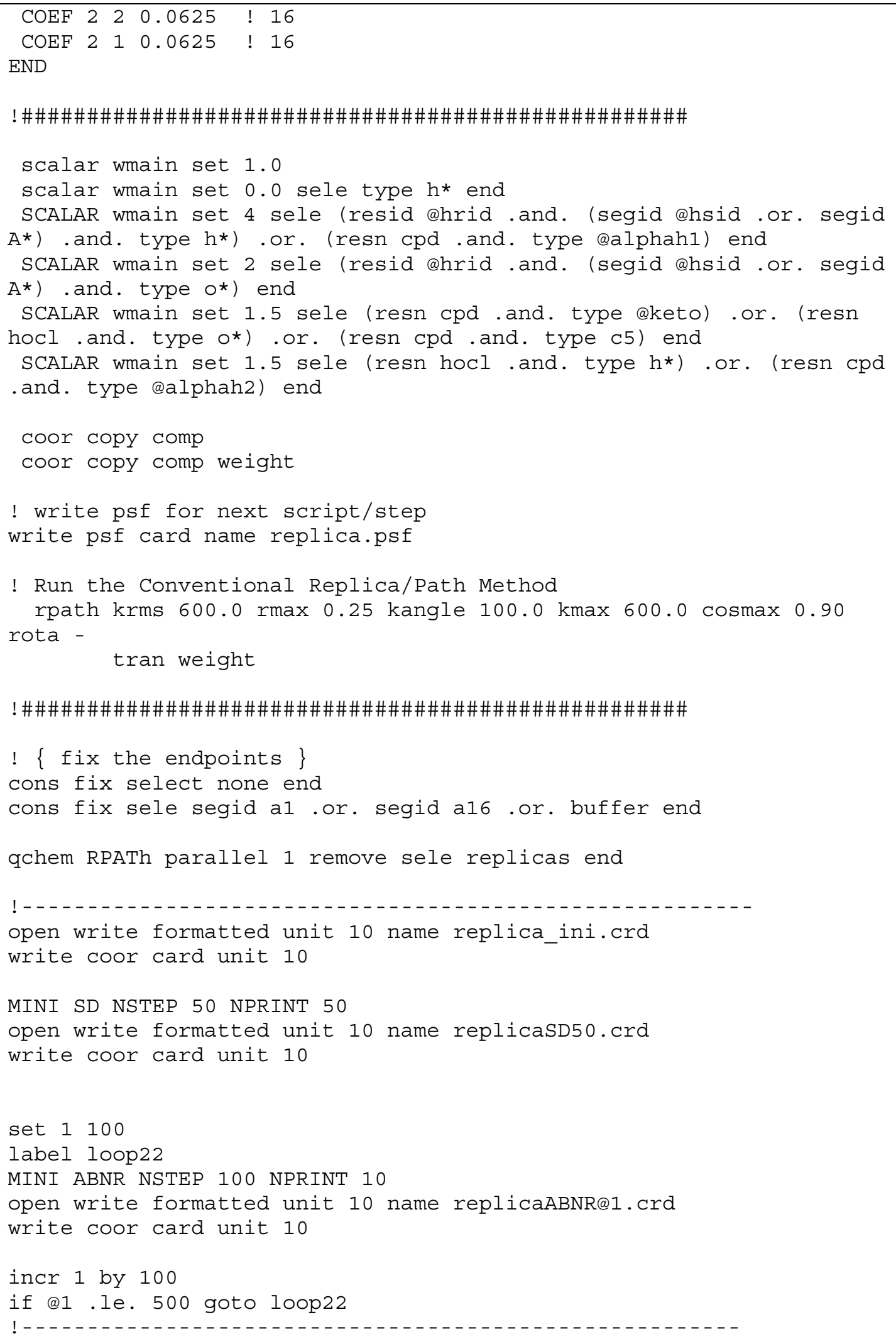




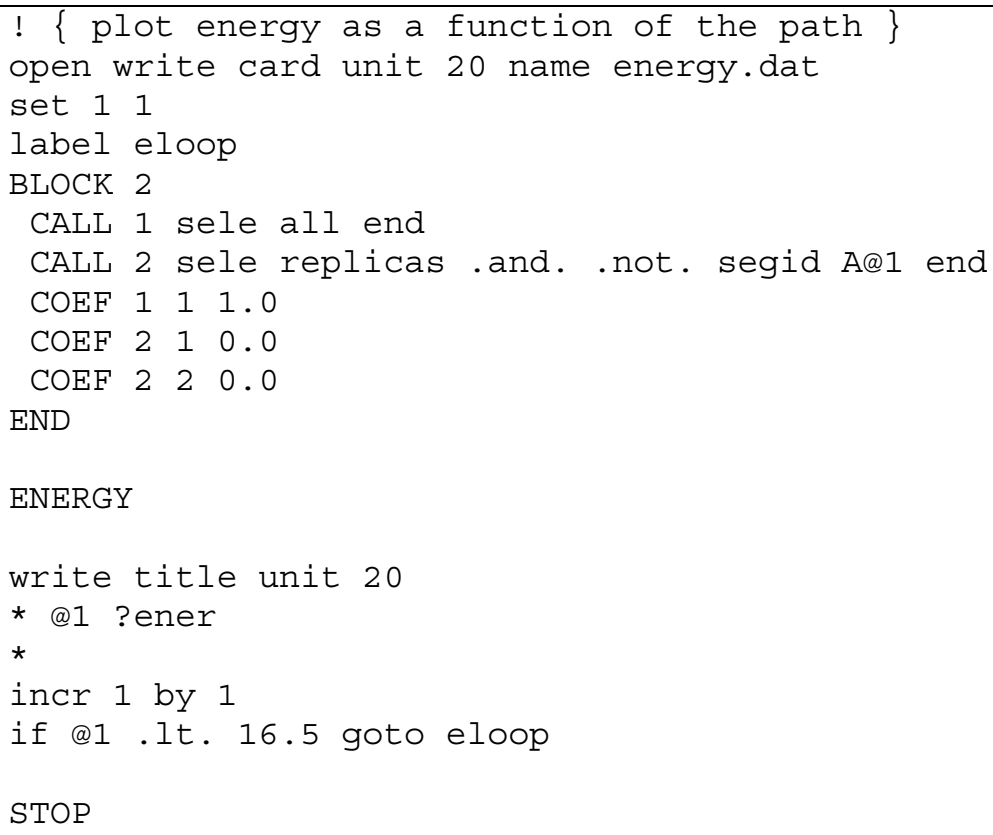

Table 45: Script for mapping out minimum energy path using Replica Path method with all MM waters fixed

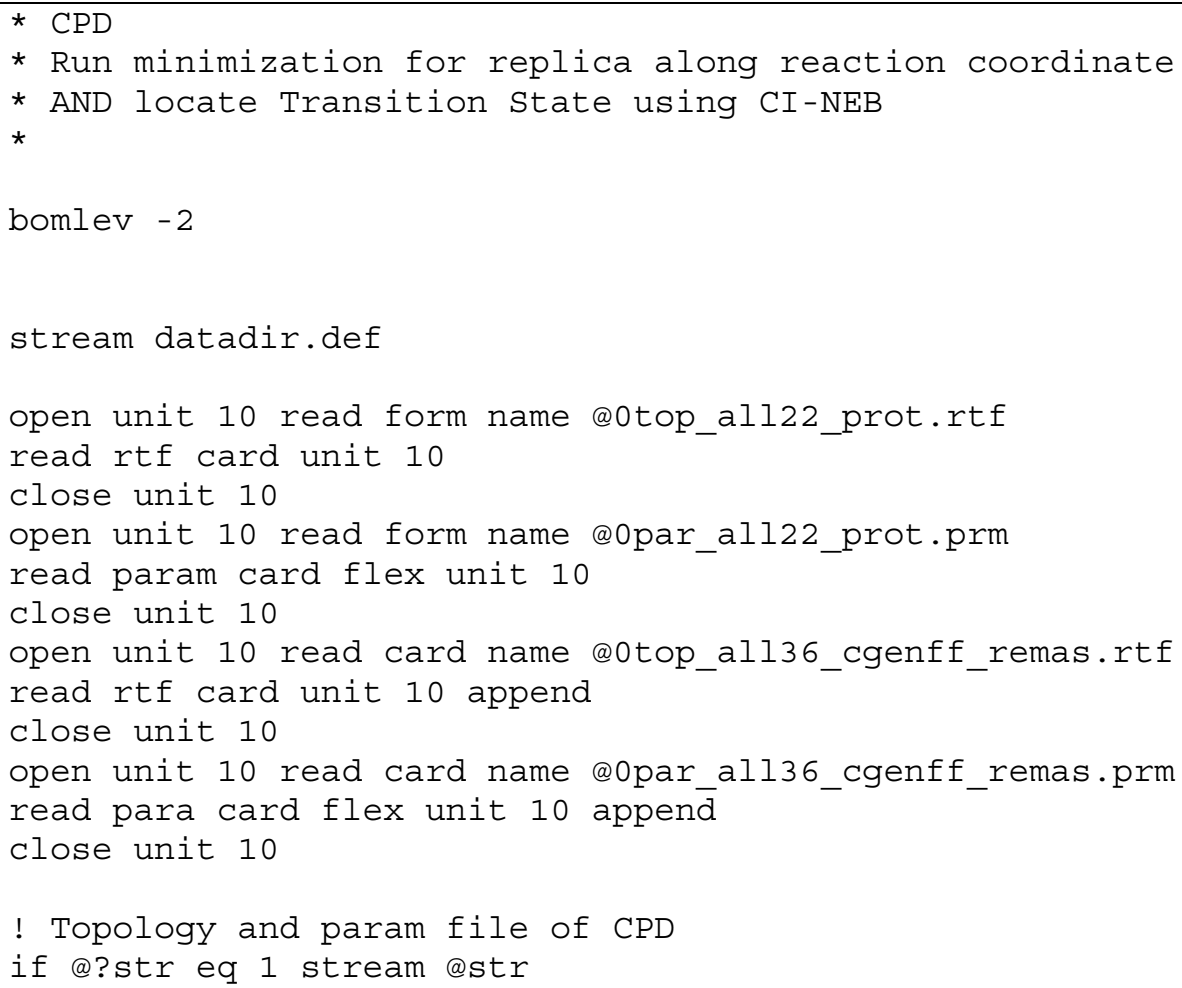




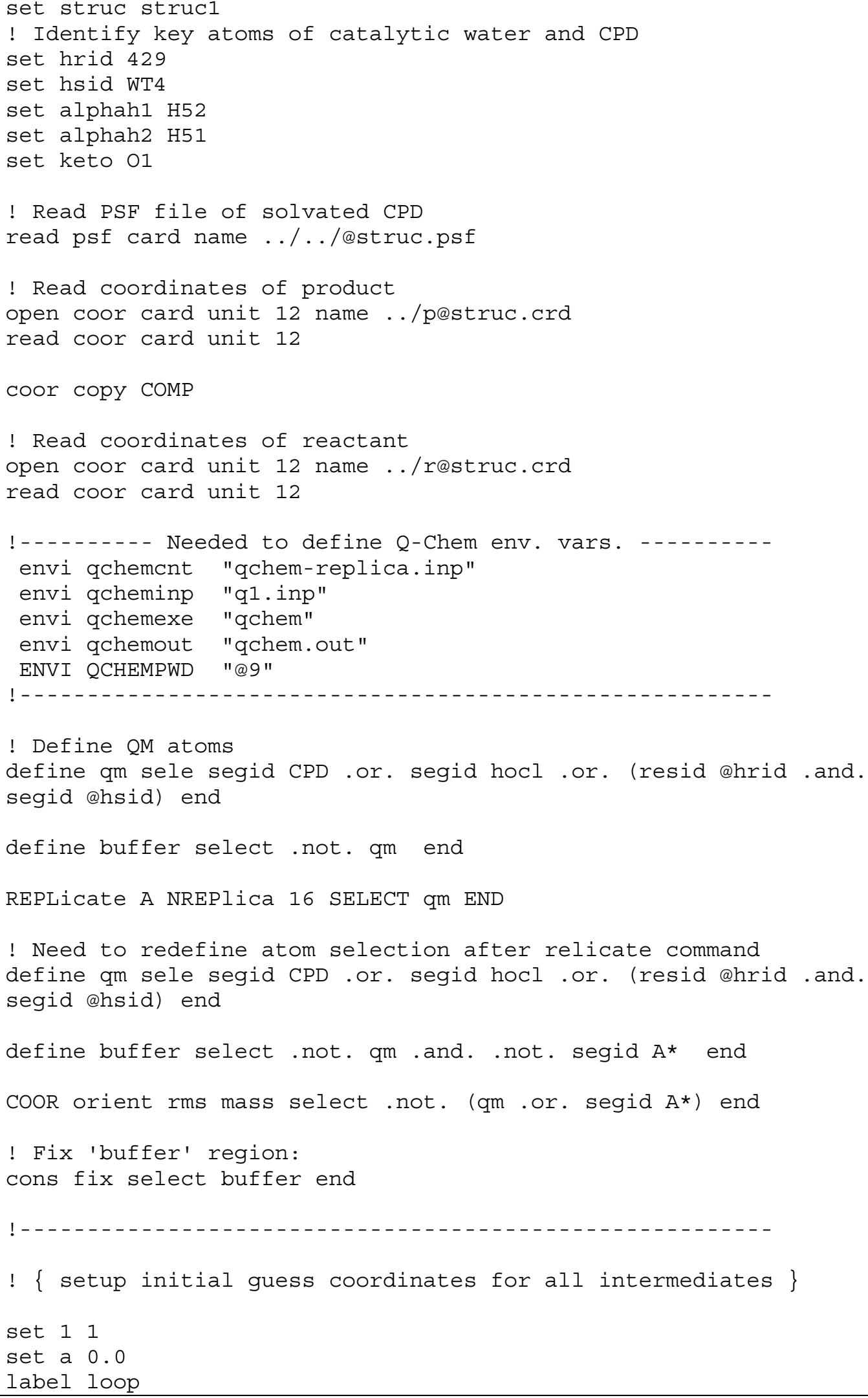




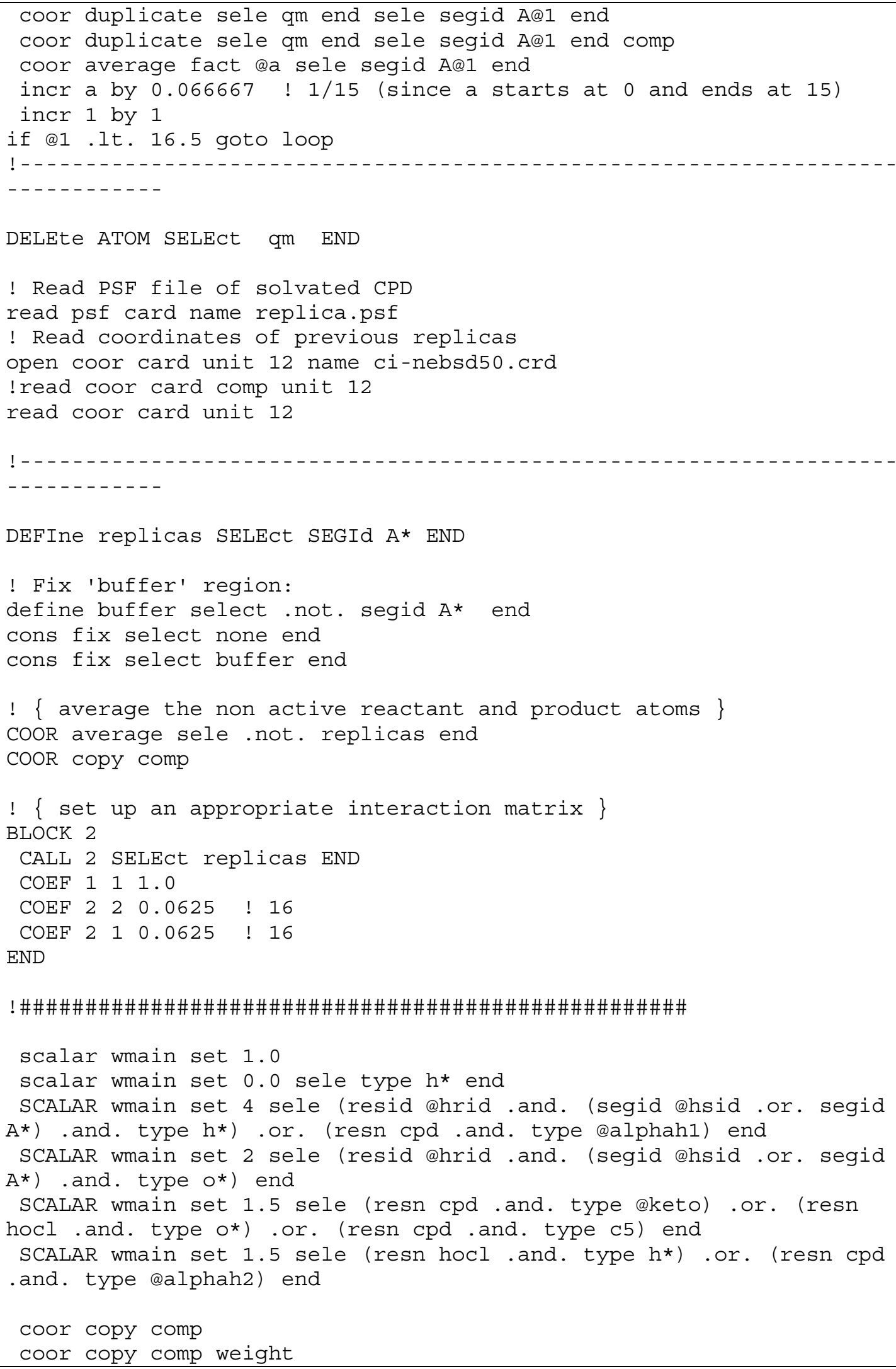




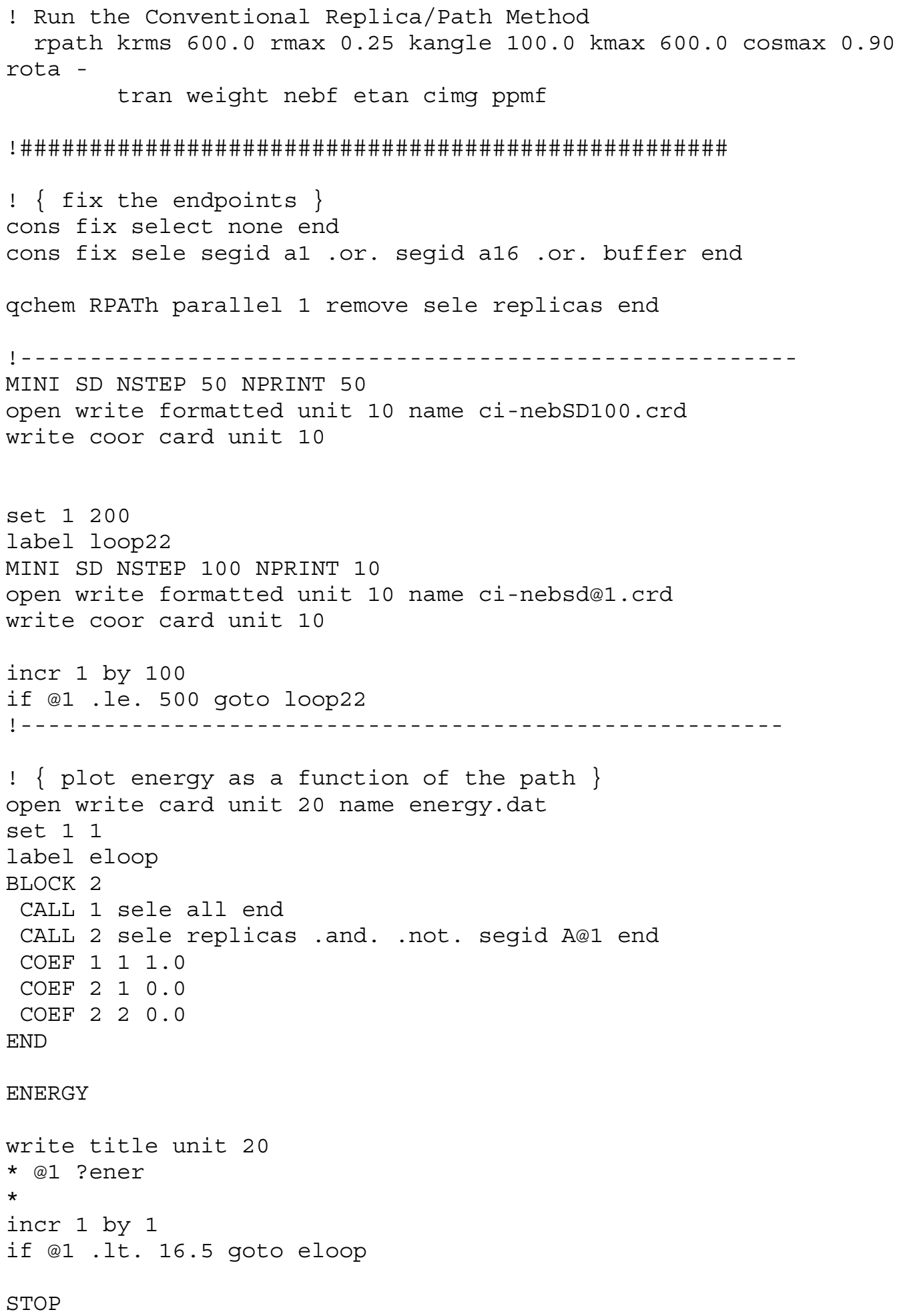

Table 46: Script for locating transition state of system using CI-NEB with all MM waters fixed 


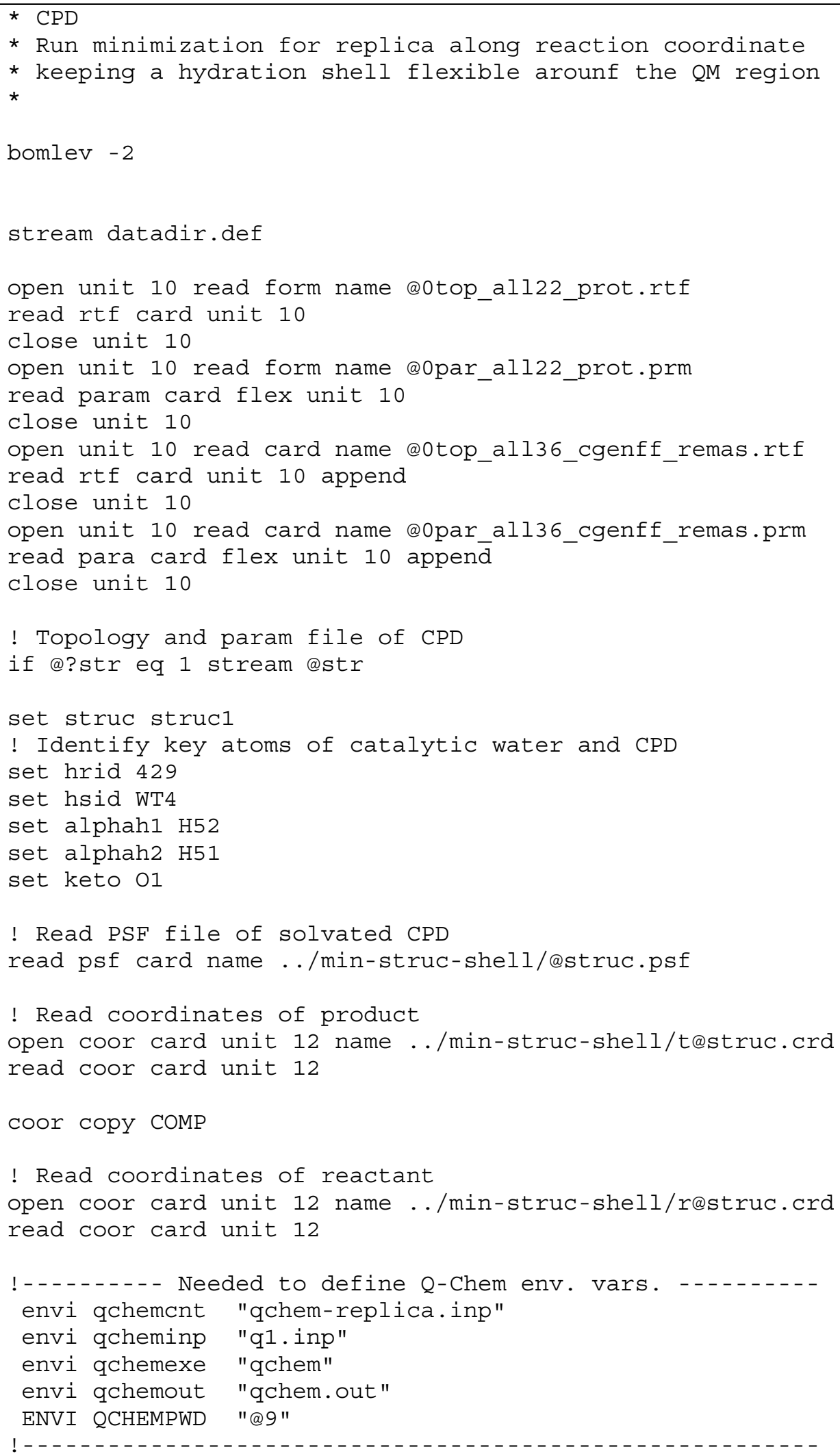




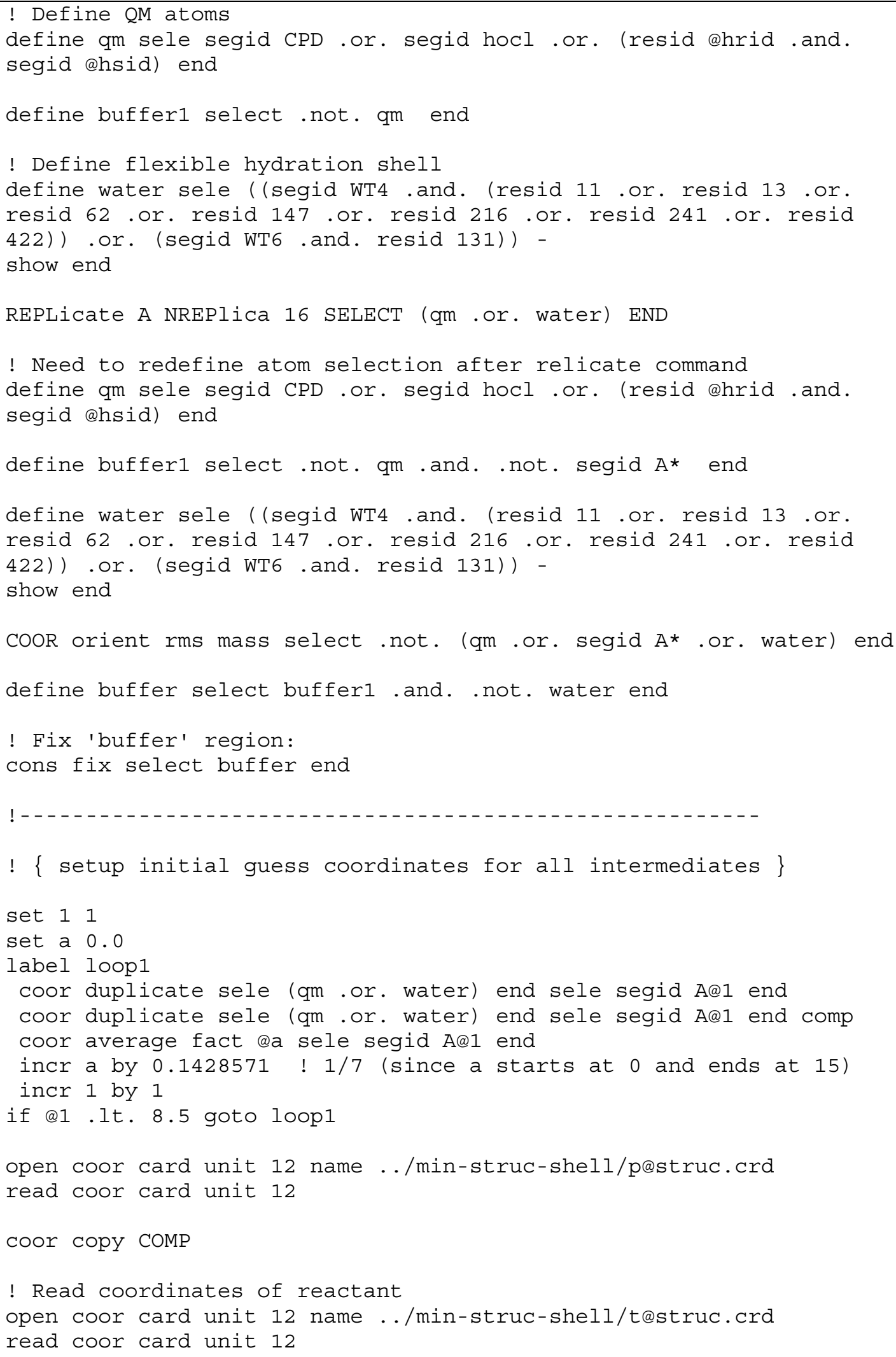




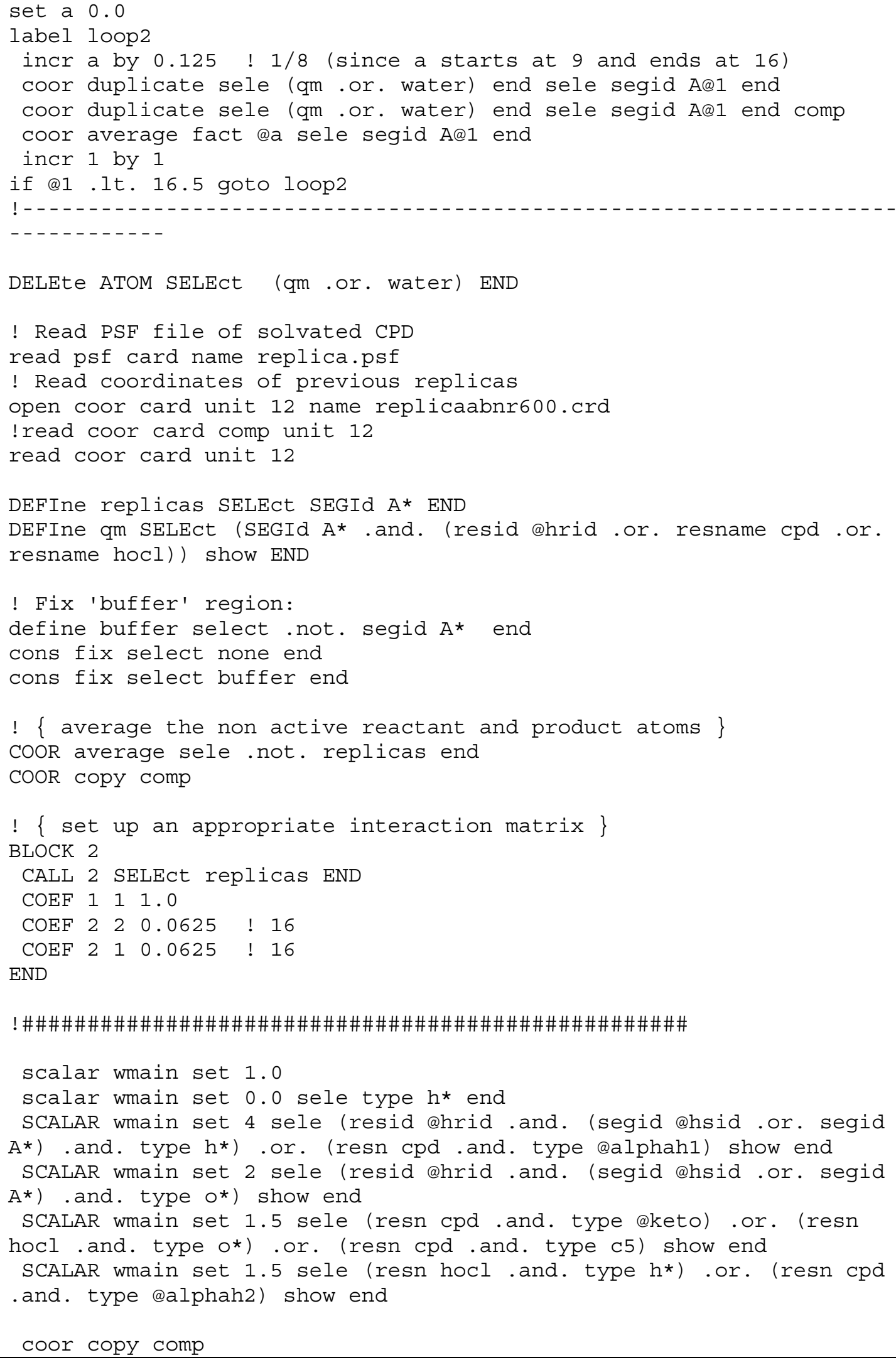




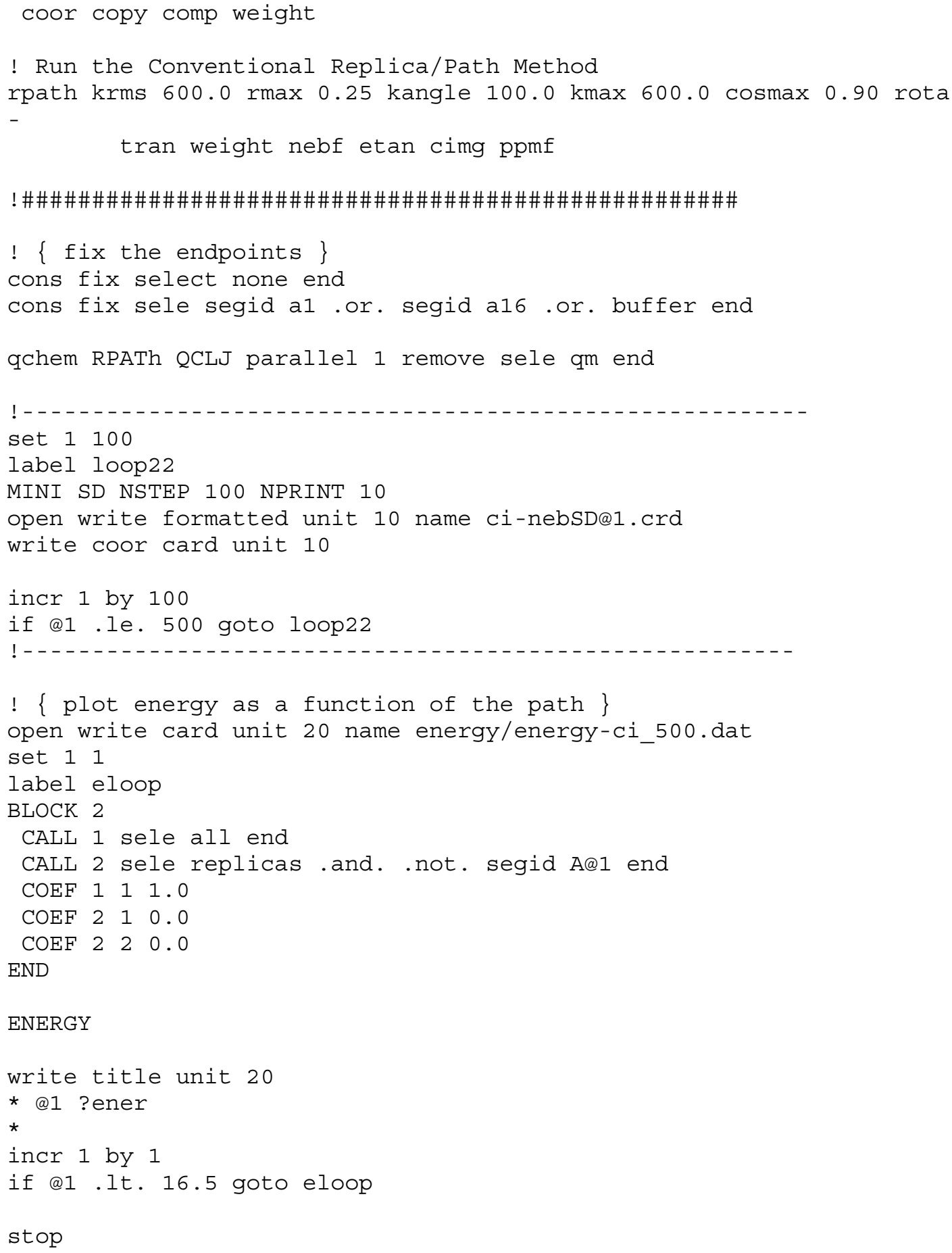

Table 47: Script for locating transition state using CI-NEB for system with flexible hydration shell 


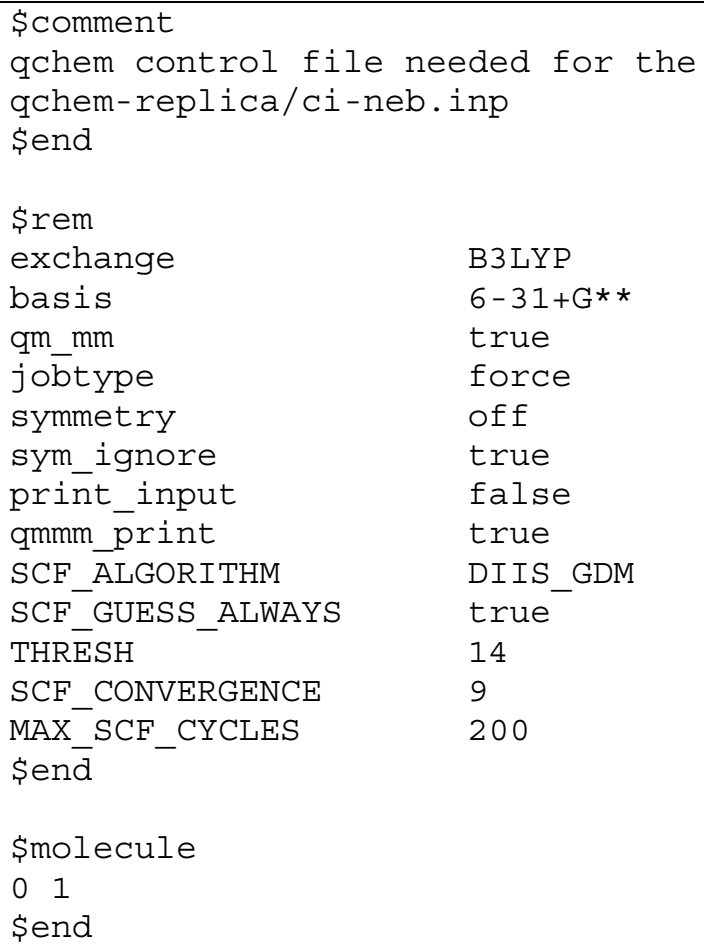

Table 48: QM script read by CHARMM to create actual script to be run by QChem. Used in Replica Path method and CI-NEB method

\begin{tabular}{|c|c|c|c|}
\hline \multicolumn{4}{|l|}{ \$external_charges } \\
\hline$-12.6 \overline{6} 269371$ & -3.72031255 & -0.82295700 & -0.83400000 \\
\hline 0.00024239 & 3.15057423 & & \\
\hline-12.46711514 & -4.08620449 & -1.68400236 & 0.41700000 \\
\hline 0.00007331 & 0.40001352 & & \\
\hline-13.03480408 & -2.85588642 & -1.16791530 & 0.41700000 \\
\hline 0.00007331 & 0.40001352 & & \\
\hline-12.82328583 & -0.88125814 & -6.57305158 & -0.83400000 \\
\hline 0.00024239 & 3.15057423 & & \\
\hline-12.43442879 & -0.05557931 & -6.15039695 & 0.41700000 \\
\hline 0.00007331 & 0.40001352 & & \\
\hline-12.32563613 & -0.88686097 & -7.40489782 & 0.41700000 \\
\hline 0.00007331 & 0.40001352 & & \\
\hline-8.21232033 & -2.74258280 & 5.04212284 & -0.83400000 \\
\hline 0.00024239 & 3.15057423 & & \\
\hline-8.40833473 & -1.77126777 & 4.86145020 & 0.41700000 \\
\hline 0.00007331 & 0.40001352 & & \\
\hline-8.27885723 & -2.76478148 & 6.04030132 & 0.41700000 \\
\hline 0.00007331 & 0.40001352 & & \\
\hline-11.67862480 & 8.36726152 & -9.01817243 & -0.83400000 \\
\hline 0.00024239 & 3.15057423 & & \\
\hline-11.98195331 & 9.23895475 & -8.73214214 & 0.41700000 \\
\hline 0.00007331 & 0.40001352 & & \\
\hline
\end{tabular}




\begin{tabular}{|c|c|c|c|}
\hline-12.57279938 & 7.96145526 & -9.25817983 & 0.41700000 \\
\hline 0.00007331 & 0.40001352 & & \\
\hline 3.44952856 & -0.70802351 & -11.04037974 & -0.83400000 \\
\hline 0.00024239 & 3.15057423 & & \\
\hline 4.19502234 & -0.41123434 & -10.54358408 & 0.41700000 \\
\hline 0.00007331 & 0.40001352 & & \\
\hline 2.76111905 & -1.03030439 & -10.34711382 & 0.41700000 \\
\hline 0.00007331 & 0.40001352 & & \\
\hline 1.74631441 & -7.57735586 & -7.82149363 & -0.83400000 \\
\hline 0.00024239 & 3.15057423 & & \\
\hline 1.88608599 & -7.97766495 & -6.95973730 & 0.41700000 \\
\hline 0.00007331 & 0.40001352 & & \\
\hline 0.95238292 & -8.00864410 & -8.19403648 & 0.41700000 \\
\hline 0.00007331 & 0.40001352 & & \\
\hline-8.90489964 & 8.30332777 & -4.33863510 & -0.83400000 \\
\hline 0.00024239 & 3.15057423 & & \\
\hline-9.47780470 & 9.03232691 & -4.47938116 & 0.41700000 \\
\hline 0.00007331 & 0.40001352 & & \\
\hline-8.57478909 & 8.57199309 & -3.45566349 & 0.41700000 \\
\hline 0.00007331 & 0.40001352 & & \\
\hline-7.58822541 & 9.37925360 & -1.99477844 & -0.83400000 \\
\hline 0.00024239 & 3.15057423 & & \\
\hline-7.33173518 & 9.14004347 & -1.09726529 & 0.41700000 \\
\hline 0.00007331 & 0.40001352 & & \\
\hline-8.25068621 & 10.13018725 & -1.80135827 & 0.41700000 \\
\hline 0.00007331 & 0.40001352 & & \\
\hline-8.04161835 & -1.21567106 & -4.70295620 & -0.83400000 \\
\hline 0.00024239 & 3.15057423 & & \\
\hline-8.63120365 & -1.92100966 & -5.04181099 & 0.41700000 \\
\hline 0.00007331 & 0.40001352 & & \\
\hline-8.11833286 & -1.30447316 & -3.73433805 & 0.41700000 \\
\hline 0.00007331 & 0.40001352 & & \\
\hline-4.79387236 & 2.43332100 & -7.43530464 & -0.83400000 \\
\hline 0.00024239 & 3.15057423 & & \\
\hline-4.88113356 & 1.47559214 & -7.60975504 & 0.41700000 \\
\hline 0.00007331 & 0.40001352 & & \\
\hline-5.71967506 & 2.66631365 & -7.72223759 & 0.41700000 \\
\hline 0.00007331 & 0.40001352 & & \\
\hline-0.78158152 & 3.00790405 & -9.29691982 & -0.83400000 \\
\hline 0.00024239 & 3.15057423 & & \\
\hline-0.92151082 & 2.10098171 & -8.98417664 & 0.41700000 \\
\hline 0.00007331 & 0.40001352 & & \\
\hline-0.55826366 & 3.04506445 & -10.22206688 & 0.41700000 \\
\hline 0.00007331 & 0.40001352 & & \\
\hline-15.28397678 & 2.52064809 & -9.35715883 & -0.83400000 \\
\hline 0.00024239 & 3.15057423 & & \\
\hline-15.33644496 & 3.37625131 & -8.87684839 & 0.41700000 \\
\hline 0.00007331 & 0.40001352 & & \\
\hline-15.90883388 & 2.12835606 & -8.72084635 & 0.41700000 \\
\hline 0.00007331 & 0.40001352 & & \\
\hline 2.06923771 & 7.52523041 & 0.79183286 & -0.83400000 \\
\hline 0.00024239 & 3.15057423 & & \\
\hline 2.85905099 & 7.62222481 & 0.25203398 & 0.41700000 \\
\hline 0.00007331 & 0.40001352 & & \\
\hline 2.15169120 & 6.52610636 & 0.92274112 & 0.41700000 \\
\hline
\end{tabular}




\begin{tabular}{|c|c|c|c|}
\hline 0.00007331 & 0.40001352 & & \\
\hline-4.75245047 & 3.31913185 & -10.09435272 & -0.83400000 \\
\hline 0.00024239 & 3.15057423 & & \\
\hline-5.01067591 & 3.26426482 & -9.13492489 & 0.41700000 \\
\hline 0.00007331 & 0.40001352 & & \\
\hline-4.87542582 & 4.27081680 & -10.23380089 & 0.41700000 \\
\hline 0.00007331 & 0.40001352 & & \\
\hline-11.10755942 & 6.90023927 & -1.86551757 & -0.83400000 \\
\hline 0.00024239 & 3.15057423 & & \\
\hline-11.67873881 & 6.29674725 & -2.37477489 & 0.41700000 \\
\hline 0.00007331 & 0.40001352 & & \\
\hline-11.42030928 & 7.74367647 & -2.26106115 & 0.41700000 \\
\hline 0.00007331 & 0.40001352 & & \\
\hline-9.95538367 & -7.17085660 & -1.88822378 & -0.83400000 \\
\hline 0.00024239 & 3.15057423 & & \\
\hline-10.81655134 & -6.88799012 & -2.17057241 & 0.41700000 \\
\hline 0.00007331 & 0.40001352 & & \\
\hline-9.58955277 & -6.44168390 & -2.42013372 & 0.41700000 \\
\hline 0.00007331 & 0.40001352 & & \\
\hline-8.20581011 & 11.30084059 & 1.41081138 & -0.83400000 \\
\hline 0.00024239 & 3.15057423 & & \\
\hline-7.73376469 & 10.50504038 & 1.12831684 & 0.41700000 \\
\hline 0.00007331 & 0.40001352 & & \\
\hline-8.92558913 & 10.99959776 & 1.94595142 & 0.41700000 \\
\hline 0.00007331 & 0.40001352 & & \\
\hline 0.16168584 & -2.01792860 & -7.50541592 & -0.83400000 \\
\hline 0.00024239 & 3.15057423 & & \\
\hline 0.34712616 & -2.90599823 & -7.07846880 & 0.41700000 \\
\hline 0.00007331 & 0.40001352 & & \\
\hline-0.60477573 & -1.85593629 & -6.95776033 & 0.41700000 \\
\hline 0.00007331 & 0.40001352 & & \\
\hline-2.12588978 & 3.02616072 & -6.71686792 & -0.83400000 \\
\hline 0.00024239 & 3.15057423 & & \\
\hline-3.03456879 & 2.88080072 & -6.92847157 & 0.41700000 \\
\hline 0.00007331 & 0.40001352 & & \\
\hline-1.76671541 & 3.39728117 & -7.53661919 & 0.41700000 \\
\hline 0.00007331 & 0.40001352 & & \\
\hline-6.14285946 & 7.88858700 & -5.11608028 & -0.83400000 \\
\hline 0.00024239 & 3.15057423 & & \\
\hline-7.08336496 & 7.83981323 & -4.87488365 & 0.41700000 \\
\hline 0.00007331 & 0.40001352 & & \\
\hline-5.97302198 & 6.92667627 & -5.08902025 & 0.41700000 \\
\hline 0.00007331 & 0.40001352 & & \\
\hline 1.81689727 & 5.69858503 & -7.30674553 & -0.83400000 \\
\hline 0.00024239 & 3.15057423 & & \\
\hline 1.59058917 & 4.86141920 & -7.68436670 & 0.41700000 \\
\hline 0.00007331 & 0.40001352 & & \\
\hline 2.70646715 & 5.43788433 & -6.92526436 & 0.41700000 \\
\hline 0.00007331 & 0.40001352 & & \\
\hline-6.23954790 & 5.53375944 & -9.18444555 & -0.83400000 \\
\hline 0.00024239 & 3.15057423 & & \\
\hline-6.95070474 & 6.09495386 & -8.81293361 & 0.41700000 \\
\hline 0.00007331 & 0.40001352 & & \\
\hline-6.49491709 & 4.66287192 & -8.81128567 & 0.41700000 \\
\hline 0.00007331 & 0.40001352 & & \\
\hline
\end{tabular}




\begin{tabular}{|c|c|c|c|}
\hline-9.15484623 & 11.43463251 & -1.47783427 & -0.83400000 \\
\hline 0.00024239 & 3.15057423 & & \\
\hline-8.91523747 & 12.08645365 & -0.77841572 & 0.41700000 \\
\hline 0.00007331 & 0.40001352 & & \\
\hline-10.12292866 & $\quad 11.60295698$ & -1.57418231 & 0.41700000 \\
\hline 0.00007331 & 0.40001352 & & \\
\hline-4.96940088 & 7.32975769 & 5.59079790 & -0.83400000 \\
\hline 0.00024239 & 3.15057423 & & \\
\hline-4.97037077 & 8.25142002 & 5.25582504 & 0.41700000 \\
\hline 0.00007331 & 0.40001352 & & \\
\hline-4.40525436 & 6.91930676 & 4.96578026 & 0.41700000 \\
\hline 0.00007331 & 0.40001352 & & \\
\hline-0.26684002 & 10.00807020 & -10.06604914 & -0.83400000 \\
\hline 0.00024239 & 3.15057423 & & \\
\hline 0.03129144 & 9.58866331 & -9.17263989 & 0.41700000 \\
\hline 0.00007331 & 0.40001352 & & \\
\hline-0.95131163 & 10.56023714 & -9.62617354 & 0.41700000 \\
\hline 0.00007331 & 0.40001352 & & \\
\hline-11.15907404 & 5.46717755 & 0.61493973 & -0.83400000 \\
\hline 0.00024239 & 3.15057423 & & \\
\hline-11.00866053 & 5.88342504 & -0.24188514 & 0.41700000 \\
\hline 0.00007331 & 0.40001352 & & \\
\hline-10.59244559 & 5.96438555 & 1.28520636 & 0.41700000 \\
\hline 0.00007331 & 0.40001352 & & \\
\hline-4.83281708 & -6.92203426 & -1.25783682 & -0.83400000 \\
\hline 0.00024239 & 3.15057423 & & \\
\hline-4.91476154 & -6.10188293 & -0.64053756 & 0.41700000 \\
\hline 0.00007331 & 0.40001352 & & \\
\hline-3.88136363 & -6.92576265 & -1.46580851 & 0.41700000 \\
\hline 0.00007331 & 0.40001352 & & \\
\hline-1.64866905 & 9.96153948 & -6.94652442 & -0.83400000 \\
\hline 0.00024239 & 3.15057423 & & \\
\hline-1.67398457 & 10.90520594 & -7.14385967 & 0.41700000 \\
\hline 0.00007331 & 0.40001352 & & \\
\hline-1.66991572 & 10.06001780 & -5.95639495 & 0.41700000 \\
\hline 0.00007331 & 0.40001352 & & \\
\hline-0.23070198 & 5.22515583 & -2.17702746 & -0.83400000 \\
\hline 0.00024239 & 3.15057423 & & \\
\hline-0.68355227 & 5.50536346 & -3.00527048 & 0.41700000 \\
\hline 0.00007331 & 0.40001352 & & \\
\hline 0.65467203 & 5.28325462 & -2.50035000 & 0.41700000 \\
\hline 0.00007331 & 0.40001352 & & \\
\hline 0.89187348 & 0.68845546 & -4.60773897 & -0.83400000 \\
\hline 0.00024239 & 3.15057423 & & \\
\hline 1.56052935 & $\quad 1.42618001$ & -4.32142544 & 0.41700000 \\
\hline 0.00007331 & 0.40001352 & & \\
\hline 0.97568953 & 0.66868222 & -5.61498117 & 0.41700000 \\
\hline 0.00007331 & 0.40001352 & & \\
\hline-10.85094322 & -1.28269304 & -8.74101127 & -0.83400000 \\
\hline 0.00024239 & 3.15057423 & & \\
\hline-11.16956712 & -0.75009549 & -9.47861684 & 0.41700000 \\
\hline 0.00007331 & 0.40001352 & & \\
\hline-10.24647237 & -0.64405835 & -8.33378614 & 0.41700000 \\
\hline 0.00007331 & 0.40001352 & & \\
\hline-3.79904056 & $\quad-0.90402341$ & -9.47147846 & -0.83400000 \\
\hline
\end{tabular}




\begin{tabular}{|c|c|c|c|}
\hline 0.00024239 & 3.15057423 & & \\
\hline-4.06642818 & -1.54297495 & -8.79240990 & 0.41700000 \\
\hline 0.00007331 & 0.40001352 & & \\
\hline-4.66850042 & -0.62892956 & -9.77049541 & 0.41700000 \\
\hline 0.00007331 & 0.40001352 & & \\
\hline-6.40939454 & 12.73173353 & -2.70289318 & -0.83400000 \\
\hline 0.00024239 & 3.15057423 & & \\
\hline-6.89332251 & 11.97487089 & -2.36274592 & 0.41700000 \\
\hline 0.00007331 & 0.40001352 & & \\
\hline-5.60239176 & 12.65092108 & -2.20562856 & 0.41700000 \\
\hline 0.00007331 & 0.40001352 & & \\
\hline 0.18268676 & 12.24280665 & -2.97719757 & -0.83400000 \\
\hline 0.00024239 & 3.15057423 & & \\
\hline-0.32673602 & 12.72819779 & -2.30513470 & 0.41700000 \\
\hline 0.00007331 & 0.40001352 & & \\
\hline-0.16427783 & 12.64990208 & -3.74899320 & 0.41700000 \\
\hline 0.00007331 & 0.40001352 & & \\
\hline 1.70999646 & 2.95553684 & -8.22914314 & -0.83400000 \\
\hline 0.00024239 & 3.15057423 & & \\
\hline 1.62235367 & 2.07860875 & -7.79374266 & 0.41700000 \\
\hline 0.00007331 & 0.40001352 & & \\
\hline 0.96318227 & 2.97343135 & -8.90891552 & 0.41700000 \\
\hline 0.00007331 & 0.40001352 & & \\
\hline-10.94326019 & -5.29858589 & 0.67193198 & -0.83400000 \\
\hline 0.00024239 & 3.15057423 & & \\
\hline-11.55381584 & -4.77557135 & 0.18947107 & 0.41700000 \\
\hline 0.00007331 & 0.40001352 & & \\
\hline-11.55175591 & -5.99943066 & 1.06350756 & 0.41700000 \\
\hline 0.00007331 & 0.40001352 & & \\
\hline-14.95601198 & -0.71559520 & -5.01344677 & -0.83400000 \\
\hline 0.00024239 & 3.15057423 & & \\
\hline-14.32886336 & -0.70913930 & -5.71967311 & 0.41700000 \\
\hline 0.00007331 & 0.40001352 & & \\
\hline-15.76153776 & -0.45086856 & -5.52604957 & 0.41700000 \\
\hline 0.00007331 & 0.40001352 & & \\
\hline-5.94910002 & 9.58284092 & 3.54445243 & -0.83400000 \\
\hline 0.00024239 & 3.15057423 & & \\
\hline-5.84677458 & 8.84972477 & 2.95315051 & 0.41700000 \\
\hline 0.00007331 & 0.40001352 & & \\
\hline-6.83834124 & $=9.41940403$ & 3.83400202 & 0.41700000 \\
\hline 0.00007331 & 0.40001352 & & \\
\hline-1.56935549 & 0.35127306 & -9.18744278 & -0.83400000 \\
\hline 0.00024239 & 3.15057423 & & \\
\hline-2.36397362 & -0.17355590 & -9.39029121 & 0.41700000 \\
\hline 0.00007331 & 0.40001352 & & \\
\hline-1.75811040 & 0.50599879 & -8.16943741 & 0.41700000 \\
\hline 0.00007331 & 0.40001352 & & \\
\hline-14.07500622 & -3.92943330 & 1.44328885 & -0.83400000 \\
\hline 0.00024239 & 3.15057423 & & \\
\hline-13.79086134 & $=-3.88089319$ & 0.51646833 & 0.41700000 \\
\hline 0.00007331 & 0.40001352 & & \\
\hline-13.71641657 & -3.12310978 & 1.84860949 & 0.41700000 \\
\hline 0.00007331 & 0.40001352 & & \\
\hline-14.20119565 & -0.15306178 & -9.65109794 & -0.83400000 \\
\hline 0.00024239 & 3.15057423 & & \\
\hline
\end{tabular}




\begin{tabular}{|c|c|c|c|}
\hline-13.31239956 & -0.01980678 & -10.02959602 & 0.41700000 \\
\hline 0.00007331 & 0.40001352 & & \\
\hline-14.23207479 & 0.72270402 & -9.13877456 & 0.41700000 \\
\hline 0.00007331 & 0.40001352 & & \\
\hline-4.26881552 & 7.71750784 & -1.41377366 & -0.83400000 \\
\hline 0.00024239 & 3.15057423 & & \\
\hline-4.41654110 & 8.23268509 & -2.18154502 & 0.41700000 \\
\hline 0.00007331 & 0.40001352 & & \\
\hline-4.30856514 & $\quad 8.49301434$ & -0.75255018 & 0.41700000 \\
\hline 0.00007331 & 0.40001352 & & \\
\hline-4.74750484 & -3.37516130 & -15.79960478 & -0.83400000 \\
\hline 0.00024239 & 3.15057423 & & \\
\hline-4.63706268 & -2.38356889 & -15.73057712 & 0.41700000 \\
\hline 0.00007331 & 0.40001352 & & \\
\hline-4.68567718 & -3.63342202 & -14.85764683 & 0.41700000 \\
\hline 0.00007331 & 0.40001352 & & \\
\hline-4.04387140 & -3.49535775 & -3.62332439 & -0.83400000 \\
\hline 0.00024239 & 3.15057423 & & \\
\hline-3.56032968 & -3.52109051 & -2.79814959 & 0.41700000 \\
\hline 0.00007331 & 0.40001352 & & \\
\hline-3.76096344 & $\quad-2.59369636$ & -3.92540073 & 0.41700000 \\
\hline 0.00007331 & 0.40001352 & & \\
\hline-4.61556101 & -1.24008286 & 4.84261656 & -0.83400000 \\
\hline 0.00024239 & 3.15057423 & & \\
\hline-5.14179945 & -0.44616830 & 4.54327583 & 0.41700000 \\
\hline 0.00007331 & 0.40001352 & & \\
\hline-5.03048182 & -1.94250596 & 4.28915596 & 0.41700000 \\
\hline 0.00007331 & 0.40001352 & & \\
\hline-1.35502505 & 2.50174856 & -4.15784693 & -0.83400000 \\
\hline 0.00024239 & 3.15057423 & & \\
\hline-0.55688095 & 2.08537936 & -4.51790762 & 0.41700000 \\
\hline 0.00007331 & 0.40001352 & & \\
\hline-1.81425250 & 2.67176390 & -5.01293898 & 0.41700000 \\
\hline 0.00007331 & 0.40001352 & & \\
\hline-4.02193737 & 3.84920478 & 1.39791763 & -0.83400000 \\
\hline 0.00024239 & 3.15057423 & & \\
\hline-3.83969712 & 4.04143286 & 0.46111229 & 0.41700000 \\
\hline 0.00007331 & 0.40001352 & & \\
\hline-3.27061915 & 4.43042898 & 1.79557204 & 0.41700000 \\
\hline 0.00007331 & 0.40001352 & & \\
\hline-4.14192867 & 4.14322472 & 6.12727976 & -0.83400000 \\
\hline 0.00024239 & 3.15057423 & & \\
\hline-3.47189999 & 4.11018562 & 6.82111549 & 0.41700000 \\
\hline 0.00007331 & 0.40001352 & & \\
\hline-3.87384987 & 4.94566154 & 5.64689922 & 0.41700000 \\
\hline 0.00007331 & 0.40001352 & & \\
\hline-6.97204511 & 4.99274978 & -12.98970979 & -0.83400000 \\
\hline 0.00024239 & 3.15057423 & & \\
\hline-7.11722868 & 5.04745182 & -13.95340984 & 0.41700000 \\
\hline 0.00007331 & 0.40001352 & & \\
\hline-6.07540624 & $\quad 5.26901039$ & -12.69357722 & 0.41700000 \\
\hline 0.00007331 & 0.40001352 & & \\
\hline-11.15246794 & $\quad-0.81246515$ & -1.01006361 & -0.83400000 \\
\hline 0.00024239 & 3.15057423 & & \\
\hline-11.65789435 & $\quad-0.15057273$ & -0.48734994 & 0.41700000 \\
\hline
\end{tabular}




\begin{tabular}{|c|c|c|c|}
\hline 0.00007331 & 0.40001352 & & \\
\hline-10.98611185 & -1.53026434 & -0.37222715 & 0.41700000 \\
\hline 0.00007331 & 0.40001352 & & \\
\hline-10.49845339 & -2.72003377 & -4.31350005 & -0.83400000 \\
\hline 0.00024239 & 3.15057423 & & \\
\hline-10.03901196 & -3.47029699 & -3.88716806 & 0.41700000 \\
\hline 0.00007331 & 0.40001352 & & \\
\hline-11.21078218 & -3.11810888 & -4.82077945 & 0.41700000 \\
\hline 0.00007331 & 0.40001352 & & \\
\hline-10.43672371 & -2.58069944 & 0.94477940 & -0.83400000 \\
\hline 0.00024239 & 3.15057423 & & \\
\hline-10.76564312 & -2.61942196 & 1.87792277 & 0.41700000 \\
\hline 0.00007331 & 0.40001352 & & \\
\hline-10.24156666 & -3.48802614 & 0.75041306 & 0.41700000 \\
\hline 0.00007331 & 0.40001352 & & \\
\hline 0.91760345 & 9.73693964 & -7.59769754 & -0.83400000 \\
\hline 0.00024239 & 3.15057423 & & \\
\hline 1.23726268 & 10.49232313 & -7.09701375 & 0.41700000 \\
\hline 0.00007331 & 0.40001352 & & \\
\hline-0.02289228 & 9.75407335 & -7.26275258 & 0.41700000 \\
\hline 0.00007331 & 0.40001352 & & \\
\hline-5.99805737 & 0.84667850 & 3.87888217 & -0.83400000 \\
\hline 0.00024239 & 3.15057423 & & \\
\hline-5.94073153 & 1.76076257 & 4.15010500 & 0.41700000 \\
\hline 0.00007331 & 0.40001352 & & \\
\hline-6.92460632 & 0.94163722 & 3.55427098 & 0.41700000 \\
\hline 0.00007331 & 0.40001352 & & \\
\hline-2.01993370 & -2.41292572 & 4.75738716 & -0.83400000 \\
\hline 0.00024239 & 3.15057423 & & \\
\hline-2.73513818 & -1.84698534 & 5.13407993 & 0.41700000 \\
\hline 0.00007331 & 0.40001352 & & \\
\hline-2.52957535 & -3.24370980 & 4.59012318 & 0.41700000 \\
\hline 0.00007331 & 0.40001352 & & \\
\hline-5.43073265 & $\quad 10.00289938$ & -6.50841932 & -0.83400000 \\
\hline 0.00024239 & 3.15057423 & & \\
\hline-5.61131529 & 9.02838728 & -6.27480845 & 0.41700000 \\
\hline 0.00007331 & 0.40001352 & & \\
\hline-4.66234718 & $\quad 10.16112539$ & -5.88907926 & 0.41700000 \\
\hline 0.00007331 & 0.40001352 & & \\
\hline-11.03087748 & -7.08904613 & -4.87601007 & -0.83400000 \\
\hline 0.00024239 & 3.15057423 & & \\
\hline-10.90402795 & -6.16890395 & -4.59643758 & 0.41700000 \\
\hline 0.00007331 & 0.40001352 & & \\
\hline-10.80614556 & -7.09166301 & -5.84391130 & 0.41700000 \\
\hline 0.00007331 & 0.40001352 & & \\
\hline-3.63956523 & -3.36325860 & -0.87198746 & -0.83400000 \\
\hline 0.00024239 & 3.15057423 & & \\
\hline-3.94332099 & -2.65172744 & -0.25449085 & 0.41700000 \\
\hline 0.00007331 & 0.40001352 & & \\
\hline-3.69248080 & -4.07021904 & -0.17554607 & 0.41700000 \\
\hline 0.00007331 & 0.40001352 & & \\
\hline-10.08251974 & $\quad 5.72401301$ & 4.71755896 & -0.83400000 \\
\hline 0.00024239 & 3.15057423 & & \\
\hline-9.62992594 & $\quad 4.95266090$ & 4.32068686 & 0.41700000 \\
\hline 0.00007331 & 0.40001352 & & \\
\hline
\end{tabular}




\begin{tabular}{|c|c|c|c|}
\hline-10.86702463 & 5.21120922 & 5.15894978 & 0.41700000 \\
\hline 0.00007331 & 0.40001352 & & \\
\hline-4.13924782 & 11.85287878 & -1.60033063 & -0.83400000 \\
\hline 0.00024239 & 3.15057423 & & \\
\hline-4.31139831 & 11.72317812 & -0.65656475 & 0.41700000 \\
\hline 0.00007331 & 0.40001352 & & \\
\hline-4.23945564 & 10.91515658 & -1.76205759 & 0.41700000 \\
\hline $0.00007331 \quad 0$ & 0.40001352 & & \\
\hline-4.39291442 & -4.17281164 & -13.18898035 & -0.83400000 \\
\hline 0.00024239 & 3.15057423 & & \\
\hline-3.98017515 & -3.53256524 & -12.49230824 & 0.41700000 \\
\hline 0.00007331 & 0.40001352 & & \\
\hline-3.69411672 & -4.87217391 & -13.18280781 & 0.41700000 \\
\hline 0.00007331 & 0.40001352 & & \\
\hline 1.20678234 & -5.24383259 & -8.92506409 & -0.83400000 \\
\hline 0.00024239 & 3.15057423 & & \\
\hline 1.35684121 & -6.17150831 & -8.64550972 & 0.41700000 \\
\hline $0.00007331 \quad 0$ & 0.40001352 & & \\
\hline 1.96638489 & -5.15202427 & -9.56377506 & 0.41700000 \\
\hline 0.00007331 & 0.40001352 & & \\
\hline-9.16294765 & 1.08909702 & -3.71860242 & -0.83400000 \\
\hline 0.00024239 & 3.15057423 & & \\
\hline-8.78751564 & 0.20517330 & -3.61345363 & 0.41700000 \\
\hline 0.00007331 & 0.40001352 & & \\
\hline-10.02747917 & 0.90368420 & -4.07745409 & 0.41700000 \\
\hline $0.00007331 \quad 0$ & 0.40001352 & & \\
\hline-0.67135024 & 2.72109532 & 6.92797995 & -0.83400000 \\
\hline 0.00024239 & 3.15057423 & & \\
\hline-0.64405292 & 2.37443161 & 7.87056541 & 0.41700000 \\
\hline $0.00007331 \quad 0$ & 0.40001352 & & \\
\hline-0.21395230 & 3.57453251 & 7.07770300 & 0.41700000 \\
\hline $0.00007331 \quad 0$ & 0.40001352 & & \\
\hline-10.68309562 & 3.89056596 & -14.85120957 & -0.83400000 \\
\hline 0.00024239 & 3.15057423 & & \\
\hline-11.01002043 & 4.37402638 & -14.05275243 & 0.41700000 \\
\hline 0.00007331 & 0.40001352 & & \\
\hline-11.09756201 & 4.42782291 & -15.53460645 & 0.41700000 \\
\hline 0.00007331 & 0.40001352 & & \\
\hline-1.02736950 & -3.80126142 & 1.77678549 & -0.83400000 \\
\hline 0.00024239 & 3.15057423 & & \\
\hline-0.28711194 & -4.12946701 & 2.31694460 & 0.41700000 \\
\hline 0.00007331 & 0.40001352 & & \\
\hline-1.66594553 & -3.93872166 & 2.52733207 & 0.41700000 \\
\hline 0.00007331 & 0.40001352 & & \\
\hline 1.71680295 & 8.01037121 & -4.37776756 & -0.83400000 \\
\hline 0.00024239 & 3.15057423 & & \\
\hline 2.63868952 & 8.24894619 & -4.36792231 & 0.41700000 \\
\hline 0.00007331 & 0.40001352 & & \\
\hline 1.45998514 & 8.57782555 & -3.65151715 & 0.41700000 \\
\hline $0.00007331 \quad 0$ & 0.40001352 & & \\
\hline 1.53728318 & 9.93886471 & -2.37570119 & -0.83400000 \\
\hline 0.00024239 & 3.15057423 & & \\
\hline 1.00594270 & 10.64303112 & -2.76150298 & 0.41700000 \\
\hline $0.00007331 \quad 0$ & 0.40001352 & & \\
\hline 2.34552383 & 10.47169781 & -2.33500862 & 0.41700000 \\
\hline
\end{tabular}




\begin{tabular}{|c|c|c|c|}
\hline 0.00007331 & 0.40001352 & & \\
\hline-10.53553773 & $3-6.64651454$ & -7.58977521 & -0.83400000 \\
\hline 0.00024239 & 3.15057423 & & \\
\hline-10.46895374 & $4 \quad-5.78948797$ & -8.05549563 & 0.41700000 \\
\hline 0.00007331 & 0.40001352 & & \\
\hline-11.13511670 & $\quad-7.07794488$ & -8.25828088 & 0.41700000 \\
\hline 0.00007331 & 0.40001352 & & \\
\hline-15.01429571 & $1 \quad-3.79450239$ & -4.26328529 & -0.83400000 \\
\hline 0.00024239 & 3.15057423 & & \\
\hline-15.81602348 & $3 \quad-3.94996084$ & -4.77125896 & 0.41700000 \\
\hline 0.00007331 & 0.40001352 & & \\
\hline-15.03899062 & $2 \quad-4.56679166$ & -3.66648496 & 0.41700000 \\
\hline 0.00007331 & 0.40001352 & & \\
\hline-5.38185132 & $2 \quad-6.40675128$ & -10.09477533 & -0.83400000 \\
\hline 0.00024239 & 3.15057423 & & \\
\hline-5.30530752 & $2 \quad-6.91480697$ & -9.30909980 & 0.41700000 \\
\hline 0.00007331 & 0.40001352 & & \\
\hline-5.07502664 & $4 \quad-7.15343488$ & -10.65338577 & 0.41700000 \\
\hline 0.00007331 & 0.40001352 & & \\
\hline-0.06777705 & $5 \quad-1.54545724$ & 3.20003581 & -0.83400000 \\
\hline 0.00024239 & 3.15057423 & & \\
\hline-0.67194760 & $\quad-1.83165503$ & 3.93613744 & 0.41700000 \\
\hline 0.00007331 & 0.40001352 & & \\
\hline-0.39186412 & $2 \quad-2.25184846$ & 2.60409546 & 0.41700000 \\
\hline 0.00007331 & 0.40001352 & & \\
\hline-10.30944066 & $5 \quad 10.89215967$ & -4.46063272 & -0.83400000 \\
\hline 0.00024239 & 3.15057423 & & \\
\hline-10.69502835 & $5 \quad 11.15455458$ & -3.60946570 & 0.41700000 \\
\hline 0.00007331 & 0.40001352 & & \\
\hline-9.70807843 & $3 \quad 11.63192484$ & -4.68163048 & 0.41700000 \\
\hline 0.00007331 & 0.40001352 & & \\
\hline 0.61740971 & $1 \quad-4.68050623$ & -6.34114838 & -0.83400000 \\
\hline 0.00024239 & 3.15057423 & & \\
\hline 0.78671414 & $4 \quad-5.15730524$ & -7.22121811 & 0.41700000 \\
\hline 0.00007331 & 0.40001352 & & \\
\hline 0.98086965 & -5.31479073 & -5.70090866 & 0.41700000 \\
\hline 0.00007331 & 0.40001352 & & \\
\hline-8.64982002 & $2 \quad 6.60391446$ & -9.13670509 & -0.83400000 \\
\hline 0.00024239 & 3.15057423 & & \\
\hline-8.39039152 & $2 \quad 7.40284273$ & -8.62461298 & 0.41700000 \\
\hline 0.00007331 & 0.40001352 & & \\
\hline-8.59633653 & 36.94826933 & -10.04805630 & 0.41700000 \\
\hline 0.00007331 & 0.40001352 & & \\
\hline 3.02723942 & $2 \quad 5.99347262$ & -10.25929568 & -0.83400000 \\
\hline 0.00024239 & 3.15057423 & & \\
\hline 2.08526926 & $5 \quad 6.02522592$ & -10.30489561 & 0.41700000 \\
\hline 0.00007331 & 0.40001352 & & \\
\hline 3.18437706 & $6 \quad 6.67438893$ & -9.59243223 & 0.41700000 \\
\hline 0.00007331 & 0.40001352 & & \\
\hline-2.38154745 & $5 \quad-2.35321260$ & -11.34691048 & -0.83400000 \\
\hline 0.00024239 & 3.15057423 & & \\
\hline-2.93011117 & $7 \quad-1.79772305$ & -10.80638409 & 0.41700000 \\
\hline 0.00007331 & 0.40001352 & & \\
\hline-1.74729598 & $3 \quad-2.70422769$ & -10.63128185 & 0.41700000 \\
\hline 0.00007331 & 0.40001352 & & \\
\hline
\end{tabular}




\begin{tabular}{|c|c|c|c|}
\hline-12.44829056 & 1.53020648 & -2.10830064 & -0.83400000 \\
\hline 0.00024239 & 3.15057423 & & \\
\hline-12.23021910 & 0.81421022 & -2.72519775 & 0.41700000 \\
\hline 0.00007331 & 0.40001352 & & \\
\hline-13.35727379 & 1.31450180 & -1.91980024 & 0.41700000 \\
\hline 0.00007331 & 0.40001352 & & \\
\hline 3.43269563 & -2.69687724 & -0.10293386 & -0.83400000 \\
\hline 0.00024239 & 3.15057423 & & \\
\hline 2.54266810 & -3.08579445 & 0.04740809 & 0.41700000 \\
\hline 0.00007331 & 0.40001352 & & \\
\hline 3.14372587 & -1.90356135 & -0.56189650 & 0.41700000 \\
\hline 0.00007331 & 0.40001352 & & \\
\hline 2.08547902 & 2.45265818 & 2.48892140 & -0.83400000 \\
\hline 0.00024239 & 3.15057423 & & \\
\hline 2.08475614 & 1.52860451 & 2.68032646 & 0.41700000 \\
\hline 0.00007331 & 0.40001352 & & \\
\hline 1.23709583 & 2.70122695 & 2.87923908 & 0.41700000 \\
\hline 0.00007331 & 0.40001352 & & \\
\hline-0.62606150 & 1.70599651 & 4.35170889 & -0.83400000 \\
\hline 0.00024239 & 3.15057423 & & \\
\hline-1.01177561 & 1.91791201 & 5.24454880 & 0.41700000 \\
\hline 0.00007331 & 0.40001352 & & \\
\hline 0.11095714 & 1.07372582 & 4.50905991 & 0.41700000 \\
\hline 0.00007331 & 0.40001352 & & \\
\hline-8.72354126 & 0.06846704 & 4.47919989 & -0.83400000 \\
\hline 0.00024239 & 3.15057423 & & \\
\hline-8.62431431 & 0.15579160 & 5.45167637 & 0.41700000 \\
\hline 0.00007331 & 0.40001352 & & \\
\hline-9.00101471 & 0.95212418 & 4.19547892 & 0.41700000 \\
\hline 0.00007331 & 0.40001352 & & \\
\hline-8.08821886 & 8.80652806 & -7.76312845 & -0.83400000 \\
\hline 0.00024239 & 3.15057423 & & \\
\hline-8.08538168 & 8.96548381 & -6.83112448 & 0.41700000 \\
\hline 0.00007331 & 0.40001352 & & \\
\hline-8.69755762 & 9.43573841 & -7.99723547 & 0.41700000 \\
\hline 0.00007331 & 0.40001352 & & \\
\hline 1.32351474 & 8.99528412 & -11.79206106 & -0.83400000 \\
\hline 0.00024239 & 3.15057423 & & \\
\hline 1.19973521 & 8.11135249 & -11.39570257 & 0.41700000 \\
\hline 0.00007331 & 0.40001352 & & \\
\hline 0.69346599 & 9.48115878 & -11.20606730 & 0.41700000 \\
\hline 0.00007331 & 0.40001352 & & \\
\hline-2.38850546 & -4.94034481 & 3.80965996 & -0.83400000 \\
\hline 0.00024239 & 3.15057423 & & \\
\hline-1.75714445 & -5.59468269 & 4.09257984 & 0.41700000 \\
\hline 0.00007331 & 0.40001352 & & \\
\hline-3.20031834 & -5.54584837 & 3.98892403 & 0.41700000 \\
\hline 0.00007331 & 0.40001352 & & \\
\hline 4.39702923 & 3.76032346 & -11.27697775 & -0.83400000 \\
\hline 0.00024239 & 3.15057423 & & \\
\hline 3.74555446 & 3.04366396 & -11.42017958 & 0.41700000 \\
\hline 0.00007331 & 0.40001352 & & \\
\hline 3.90806332 & 4.49140419 & -10.96205828 & 0.41700000 \\
\hline 0.00007331 & 0.40001352 & & \\
\hline-4.85812473 & -1.44872844 & 0.77312088 & -0.83400000 \\
\hline
\end{tabular}




\begin{tabular}{|c|c|c|c|}
\hline 0.00024239 & 3.15057423 & & \\
\hline-4.36791420 & -0.76636475 & 1.20949137 & 0.41700000 \\
\hline 0.00007331 & 0.40001352 & & \\
\hline-5.61824656 & -1.56889963 & 1.34408581 & 0.41700000 \\
\hline 0.00007331 & 0.40001352 & & \\
\hline-9.74353950 & 9.42327854 & -10.71633022 & -0.83400000 \\
\hline 0.00024239 & 3.15057423 & & \\
\hline-10.20953052 & 8.74405369 & -10.18987100 & 0.41700000 \\
\hline 0.00007331 & 0.40001352 & & \\
\hline-9.10131710 & 8.90507975 & -11.18247001 & 0.41700000 \\
\hline 0.00007331 & 0.40001352 & & \\
\hline 2.53637052 & -1.17628205 & -2.58882594 & -0.83400000 \\
\hline 0.00024239 & 3.15057423 & & \\
\hline 2.01252079 & -0.63970524 & -2.00847268 & 0.41700000 \\
\hline 0.00007331 & 0.40001352 & & \\
\hline 2.36895680 & -0.67201531 & -3.42464590 & 0.41700000 \\
\hline 0.00007331 & 0.40001352 & & \\
\hline 1.44744551 & -5.98655796 & -1.29462314 & -0.83400000 \\
\hline 0.00024239 & 3.15057423 & & \\
\hline 1.17277491 & -5.18924332 & -0.91189516 & 0.41700000 \\
\hline 0.00007331 & 0.40001352 & & \\
\hline 2.32334924 & $=-5.80902910$ & -1.66169631 & 0.41700000 \\
\hline 0.00007331 & 0.40001352 & & \\
\hline-6.33511591 & -0.01520389 & -10.34225082 & -0.83400000 \\
\hline 0.00024239 & 3.15057423 & & \\
\hline-7.22906637 & 0.03534056 & -9.94759083 & 0.41700000 \\
\hline 0.00007331 & 0.40001352 & & \\
\hline-6.47330046 & -0.67812967 & -11.02531242 & 0.41700000 \\
\hline 0.00007331 & 0.40001352 & & \\
\hline-3.70592165 & -5.71443033 & -6.87193918 & -0.83400000 \\
\hline 0.00024239 & 3.15057423 & & \\
\hline-3.29449630 & -5.88120127 & -5.99348688 & 0.41700000 \\
\hline 0.00007331 & 0.40001352 & & \\
\hline-4.52918863 & -6.21176195 & -6.74052525 & 0.41700000 \\
\hline 0.00007331 & 0.40001352 & & \\
\hline-7.11906910 & -4.18849802 & 1.37515175 & -0.83400000 \\
\hline 0.00024239 & 3.15057423 & & \\
\hline-7.52234268 & -5.03166389 & 0.95673203 & 0.41700000 \\
\hline 0.00007331 & 0.40001352 & & \\
\hline-6.63077593 & -3.80992174 & 0.57103395 & 0.41700000 \\
\hline 0.00007331 & 0.40001352 & & \\
\hline-8.28528807 & 6.87247042 & 6.68962078 & -0.83400000 \\
\hline 0.00024239 & 3.15057423 & & \\
\hline-7.75805018 & 6.03710632 & 6.71104602 & 0.41700000 \\
\hline 0.00007331 & 0.40001352 & & \\
\hline-8.92442248 & 6.46918635 & 6.10256557 & 0.41700000 \\
\hline 0.00007331 & 0.40001352 & & \\
\hline-0.97797883 & -3.78068852 & -9.42269993 & -0.83400000 \\
\hline 0.00024239 & 3.15057423 & & \\
\hline-0.10935444 & $\quad-4.10967207$ & -9.11183834 & 0.41700000 \\
\hline 0.00007331 & 0.40001352 & & \\
\hline-1.52983975 & -4.60139513 & -9.34214687 & 0.41700000 \\
\hline 0.00007331 & 0.40001352 & & \\
\hline-7.38302422 & 3.12178922 & -7.92789936 & -0.83400000 \\
\hline 0.00024239 & 3.15057423 & & \\
\hline
\end{tabular}




\begin{tabular}{|c|c|c|c|}
\hline-8.27246284 & 3.35031962 & -8.32844925 & 0.41700000 \\
\hline 0.00007331 & 0.40001352 & & \\
\hline-7.70795059 & 2.82963705 & -7.04745626 & 0.41700000 \\
\hline 0.00007331 & 0.40001352 & & \\
\hline-7.88662446 & $\quad-0.96222890$ & -14.28459013 & -0.83400000 \\
\hline 0.00024239 & 3.15057423 & & \\
\hline-7.52294076 & -1.23327173 & -13.45680607 & 0.41700000 \\
\hline 0.00007331 & 0.40001352 & & \\
\hline-7.24290098 & -0.25180829 & -14.55416072 & 0.41700000 \\
\hline 0.00007331 & 0.40001352 & & \\
\hline-12.82983320 & 4.12625536 & -8.06429594 & -0.83400000 \\
\hline 0.00024239 & 3.15057423 & & \\
\hline-12.13068145 & 4.72974762 & -7.78295343 & 0.41700000 \\
\hline 0.00007331 & 0.40001352 & & \\
\hline-13.16165631 & 4.46152433 & -8.95243662 & 0.41700000 \\
\hline 0.00007331 & 0.40001352 & & \\
\hline-4.70759682 & 12.63666985 & 0.90494724 & -0.83400000 \\
\hline 0.00024239 & 3.15057423 & & \\
\hline-5.47761945 & $\quad 12.64068434$ & 1.48255868 & 0.41700000 \\
\hline 0.00007331 & 0.40001352 & & \\
\hline-3.96486107 & 12.72035143 & 1.59445711 & 0.41700000 \\
\hline 0.00007331 & 0.40001352 & & \\
\hline-1.24187362 & 4.21707201 & 0.07136906 & -0.83400000 \\
\hline 0.00024239 & 3.15057423 & & \\
\hline-2.14307785 & 4.44525146 & -0.25578910 & 0.41700000 \\
\hline 0.00007331 & 0.40001352 & & \\
\hline-0.66912919 & 4.69755125 & -0.56244379 & 0.41700000 \\
\hline 0.00007331 & 0.40001352 & & \\
\hline-7.26906395 & 4.95614481 & 2.09572673 & -0.83400000 \\
\hline 0.00024239 & 3.15057423 & & \\
\hline-7.13686609 & 4.41562796 & 2.87547636 & 0.41700000 \\
\hline 0.00007331 & 0.40001352 & & \\
\hline-7.08530760 & 4.16864300 & 1.46855402 & 0.41700000 \\
\hline 0.00007331 & 0.40001352 & & \\
\hline 3.01701570 & -4.49612761 & -10.81339359 & -0.83400000 \\
\hline 0.00024239 & 3.15057423 & & \\
\hline 2.50256014 & $\quad-3.82992887$ & -11.23882484 & 0.41700000 \\
\hline 0.00007331 & 0.40001352 & & \\
\hline 2.92421103 & -5.16518164 & -11.55401802 & 0.41700000 \\
\hline 0.00007331 & 0.40001352 & & \\
\hline-12.21378527 & -5.08707012 & -3.07905973 & -0.83400000 \\
\hline 0.00024239 & 3.15057423 & & \\
\hline-12.14048893 & -4.51026452 & -3.82017339 & 0.41700000 \\
\hline 0.00007331 & 0.40001352 & & \\
\hline-12.40157674 & $\quad-5.90906490$ & -3.49503053 & 0.41700000 \\
\hline 0.00007331 & 0.40001352 & & \\
\hline-10.05143850 & 1.94935616 & -10.18584316 & -0.83400000 \\
\hline 0.00024239 & 3.15057423 & & \\
\hline-9.50594919 & 2.15841588 & -10.95662229 & 0.41700000 \\
\hline 0.00007331 & 0.40001352 & & \\
\hline-10.07889145 & $\quad 2.79037722$ & -9.62124269 & 0.41700000 \\
\hline 0.00007331 & 0.40001352 & & \\
\hline-8.82584394 & $\quad-9.10530961$ & -4.30116571 & -0.83400000 \\
\hline 0.00024239 & 3.15057423 & & \\
\hline-9.49099816 & $\quad-8.40333713$ & -4.14527286 & 0.41700000 \\
\hline
\end{tabular}




\begin{tabular}{|c|c|c|c|}
\hline 0.00007331 & 0.40001352 & & \\
\hline-8.32374943 & $3 \quad-8.85117400$ & -5.12318147 & 0.41700000 \\
\hline 0.00007331 & 0.40001352 & & \\
\hline 1.37454128 & $3 \quad 0.25868979$ & -7.27763844 & -0.83400000 \\
\hline 0.00024239 & 3.15057423 & & \\
\hline 2.33860111 & $1 \quad 0.10850611$ & -7.38796854 & 0.41700000 \\
\hline 0.00007331 & 0.40001352 & & \\
\hline 0.99464637 & $\begin{array}{ll}7 & -0.53343081\end{array}$ & -7.71353245 & 0.41700000 \\
\hline 0.00007331 & 0.40001352 & & \\
\hline-11.18325753 & $3 \quad-4.23153699$ & -8.70698727 & -0.83400000 \\
\hline 0.00024239 & 3.15057423 & & \\
\hline-10.78524161 & $1 \quad-4.06742681$ & -9.57790888 & 0.41700000 \\
\hline 0.00007331 & 0.40001352 & & \\
\hline-11.25484593 & $3 \quad-3.36790002$ & -8.24308670 & 0.41700000 \\
\hline 0.00007331 & 0.40001352 & & \\
\hline-7.12915611 & $1 \quad 8.89290428$ & 0.73002899 & -0.83400000 \\
\hline 0.00024239 & 3.15057423 & & \\
\hline-6.33329964 & 48.44900608 & 1.14337349 & 0.41700000 \\
\hline 0.00007331 & 0.40001352 & & \\
\hline-7.88682985 & $5 \quad 8.37209320$ & 1.08534729 & 0.41700000 \\
\hline 0.00007331 & 0.40001352 & & \\
\hline 5.38012457 & $7 \quad-0.54735911$ & -2.27368593 & -0.83400000 \\
\hline 0.00024239 & 3.15057423 & & \\
\hline 4.51870298 & $3 \quad-0.15049253$ & -1.96952999 & 0.41700000 \\
\hline 0.00007331 & 0.40001352 & & \\
\hline 5.07495451 & $1 \quad-0.94536340$ & -3.09382677 & 0.41700000 \\
\hline 0.00007331 & 0.40001352 & & \\
\hline-3.46732783 & 34.77845144 & -3.82093167 & -0.83400000 \\
\hline 0.00024239 & 3.15057423 & & \\
\hline-3.38476062 & $2 \quad 5.57009029$ & -4.33427954 & 0.41700000 \\
\hline 0.00007331 & 0.40001352 & & \\
\hline-2.67004585 & $5 \quad 4.25333261$ & -3.99563122 & 0.41700000 \\
\hline 0.00007331 & 0.40001352 & & \\
\hline-14.55496531 & 14.38153252 & -5.58311479 & -0.83400000 \\
\hline 0.00024239 & 3.15057423 & & \\
\hline-13.80156510 & 3.96126088 & -6.13437097 & 0.41700000 \\
\hline 0.00007331 & 0.40001352 & & \\
\hline-14.31428247 & $7 \quad 5.30897459$ & -5.42915075 & 0.41700000 \\
\hline 0.00007331 & 0.40001352 & & \\
\hline-2.72425508 & $3 \quad 6.60446930$ & 1.62740326 & -0.83400000 \\
\hline 0.00024239 & 3.15057423 & & \\
\hline-2.32749271 & $1 \quad 7.46156549$ & 1.71987069 & 0.41700000 \\
\hline 0.00007331 & 0.40001352 & & \\
\hline-2.76245975 & $5 \quad 6.48467875$ & 0.67839438 & 0.41700000 \\
\hline 0.00007331 & 0.40001352 & & \\
\hline 3.33107523 & $3 \quad 8.43439345$ & -8.54588148 & -0.83400000 \\
\hline 0.00024239 & 3.15057423 & & \\
\hline 2.40179531 & $1 \quad 8.72522597$ & -8.24057886 & 0.41700000 \\
\hline 0.00007331 & 0.40001352 & & \\
\hline 3.17592756 & $5 \quad 8.79769282$ & -9.48630545 & 0.41700000 \\
\hline 0.00007331 & 0.40001352 & & \\
\hline-1.44419694 & $4 \quad-0.15008463$ & 0.90180939 & -0.83400000 \\
\hline 0.00024239 & 3.15057423 & & \\
\hline-0.93585372 & $2 \quad 0.05823188$ & 1.70593786 & 0.41700000 \\
\hline 0.00007331 & 0.40001352 & & \\
\hline
\end{tabular}




\begin{tabular}{|c|c|c|c|}
\hline-2.40071201 & -0.01339579 & 1.11363053 & 0.41700000 \\
\hline 0.00007331 & 0.40001352 & & \\
\hline-14.20629809 & 0.83022694 & 2.10712771 & -0.83400000 \\
\hline 0.00024239 & 3.15057423 & & \\
\hline-14.36266777 & 1.76458744 & 1.91583304 & 0.41700000 \\
\hline 0.00007331 & 0.40001352 & & \\
\hline-15.13752863 & 0.53048877 & 2.00985317 & 0.41700000 \\
\hline 0.00007331 & 0.40001352 & & \\
\hline-6.00994361 & -3.64904512 & -9.74807466 & -0.83400000 \\
\hline 0.00024239 & 3.15057423 & & \\
\hline-6.02327836 & $\quad-4.62967027$ & -9.87929333 & 0.41700000 \\
\hline 0.00007331 & 0.40001352 & & \\
\hline-6.41130699 & -3.47952855 & -8.90855659 & 0.41700000 \\
\hline 0.00007331 & 0.40001352 & & \\
\hline-9.72279089 & 4.08046731 & -8.47114532 & -0.83400000 \\
\hline 0.00024239 & 3.15057423 & & \\
\hline-10.14164751 & 4.07075199 & -7.58354585 & 0.41700000 \\
\hline 0.00007331 & 0.40001352 & & \\
\hline-9.45448654 & $\quad 4.93826684$ & -8.67747467 & 0.41700000 \\
\hline 0.00007331 & 0.40001352 & & \\
\hline 2.36111903 & 3.35652876 & -1.40611970 & -0.83400000 \\
\hline 0.00024239 & 3.15057423 & & \\
\hline 1.52693748 & 2.91525888 & -1.57490945 & 0.41700000 \\
\hline 0.00007331 & 0.40001352 & & \\
\hline 2.59196639 & 3.60032487 & -2.29153204 & 0.41700000 \\
\hline 0.00007331 & 0.40001352 & & \\
\hline-8.00023096 & $\quad 7.26906427$ & -11.82199018 & -0.83400000 \\
\hline 0.00024239 & 3.15057423 & & \\
\hline-7.72904127 & 6.32404229 & -12.08138340 & 0.41700000 \\
\hline 0.00007331 & 0.40001352 & & \\
\hline-7.87810343 & 7.72599202 & -12.70340793 & 0.41700000 \\
\hline 0.00007331 & 0.40001352 & & \\
\hline-10.47480318 & 9.94727044 & 2.94826369 & -0.83400000 \\
\hline 0.00024239 & 3.15057423 & & \\
\hline-11.02077982 & 9.21269707 & 2.60963969 & 0.41700000 \\
\hline 0.00007331 & 0.40001352 & & \\
\hline-9.88222429 & 9.54147629 & 3.63387061 & 0.41700000 \\
\hline 0.00007331 & 0.40001352 & & \\
\hline-2.71831846 & 7.26807117 & -4.91059446 & -0.83400000 \\
\hline 0.00024239 & 3.15057423 & & \\
\hline-1.83354998 & 6.95340204 & -5.23628330 & 0.41700000 \\
\hline 0.00007331 & 0.40001352 & & \\
\hline-2.42383814 & 8.02316856 & -4.36898947 & 0.41700000 \\
\hline 0.00007331 & 0.40001352 & & \\
\hline-3.22734318 & 11.43752596 & -4.74619107 & -0.83400000 \\
\hline 0.00024239 & 3.15057423 & & \\
\hline-3.88539916 & $\quad 11.19878885$ & -4.05698187 & 0.41700000 \\
\hline 0.00007331 & 0.40001352 & & \\
\hline-3.16146670 & 12.40673325 & -4.64846705 & 0.41700000 \\
\hline 0.00007331 & 0.40001352 & & \\
\hline-12.79879639 & 8.49569516 & 1.97440962 & -0.83400000 \\
\hline 0.00024239 & 3.15057423 & & \\
\hline-13.00420162 & 9.23681168 & 1.39012246 & 0.41700000 \\
\hline 0.00007331 & 0.40001352 & & \\
\hline-13.20748398 & 7.75041036 & 1.56776909 & 0.41700000 \\
\hline
\end{tabular}




\begin{tabular}{|c|c|c|c|}
\hline 0.00007331 & 0.40001352 & & \\
\hline-0.05857183 & 9.00719643 & 0.01041870 & -0.83400000 \\
\hline 0.00024239 & 3.15057423 & & \\
\hline 0.63110948 & 8.64094925 & 0.64670825 & 0.41700000 \\
\hline 0.00007331 & 0.40001352 & & \\
\hline 0.56038725 & 9.08142948 & -0.73362136 & 0.41700000 \\
\hline 0.00007331 & 0.40001352 & & \\
\hline-7.09304714 & $=\quad-3.84997964$ & -1.75053632 & -0.83400000 \\
\hline 0.00024239 & 3.15057423 & & \\
\hline-6.13900280 & -3.86057234 & -1.52202857 & 0.41700000 \\
\hline 0.00007331 & 0.40001352 & & \\
\hline-7.32928896 & -2.90759778 & -1.78680813 & 0.41700000 \\
\hline 0.00007331 & 0.40001352 & & \\
\hline 0.51643757 & 6.08165411 & -11.06615755 & -0.83400000 \\
\hline 0.00024239 & 3.15057423 & & \\
\hline-0.45160194 & 5.91537253 & -10.87063524 & 0.41700000 \\
\hline 0.00007331 & 0.40001352 & & \\
\hline 0.61144309 & 5.56039111 & -11.89289114 & 0.41700000 \\
\hline 0.00007331 & 0.40001352 & & \\
\hline-0.30671766 & 2.19889760 & -1.54712915 & -0.83400000 \\
\hline 0.00024239 & 3.15057423 & & \\
\hline-0.69333565 & $\quad 2.51956201$ & -2.39870834 & 0.41700000 \\
\hline 0.00007331 & 0.40001352 & & \\
\hline-0.66089958 & 2.84127760 & -0.89206445 & 0.41700000 \\
\hline 0.00007331 & 0.40001352 & & \\
\hline-5.22856943 & 9.59043715 & -3.37400214 & -0.83400000 \\
\hline 0.00024239 & 3.15057423 & & \\
\hline-5.26549594 & $\quad 9.04799483$ & -4.17350060 & 0.41700000 \\
\hline 0.00007331 & 0.40001352 & & \\
\hline-6.07296972 & 9.35296080 & -2.90561656 & 0.41700000 \\
\hline 0.00007331 & 0.40001352 & & \\
\hline-5.75866365 & $\quad-5.57629251$ & -4.23175621 & -0.83400000 \\
\hline 0.00024239 & 3.15057423 & & \\
\hline-5.78828192 & -6.01294804 & -3.36458659 & 0.41700000 \\
\hline 0.00007331 & 0.40001352 & & \\
\hline-5.03877687 & -4.88105726 & -4.05672073 & 0.41700000 \\
\hline 0.00007331 & 0.40001352 & & \\
\hline-0.35178292 & $\quad 6.88002110$ & -5.96708536 & -0.83400000 \\
\hline 0.00024239 & 3.15057423 & & \\
\hline 0.05929075 & $\quad 6.69217491$ & -6.82010269 & 0.41700000 \\
\hline 0.00007331 & 0.40001352 & & \\
\hline 0.36811858 & 37.45860863 & -5.52579689 & 0.41700000 \\
\hline 0.00007331 & 0.40001352 & & \\
\hline-2.61745942 & $\quad-9.06752909$ & -7.69998706 & -0.83400000 \\
\hline 0.00024239 & 3.15057423 & & \\
\hline-2.17233766 & -9.12680710 & -6.82903446 & 0.41700000 \\
\hline 0.00007331 & 0.40001352 & & \\
\hline-1.82279123 & $\quad-9.02951897$ & -8.24847616 & 0.41700000 \\
\hline 0.00007331 & 0.40001352 & & \\
\hline-11.48333380 & 4.13632177 & -5.11629863 & -0.83400000 \\
\hline 0.00024239 & 3.15057423 & & \\
\hline-11.97418425 & 4.47260411 & -4.33130355 & 0.41700000 \\
\hline 0.00007331 & 0.40001352 & & \\
\hline-10.71379969 & 4.80816965 & -5.07707115 & 0.41700000 \\
\hline 0.00007331 & 0.40001352 & & \\
\hline
\end{tabular}




\begin{tabular}{|c|c|c|c|}
\hline-11.71560955 & -0.36630845 & -3.79901981 & -0.83400000 \\
\hline 0.00024239 & 3.15057423 & & \\
\hline-11.89199448 & -0.63953209 & -2.91745734 & 0.41700000 \\
\hline 0.00007331 & 0.40001352 & & \\
\hline-11.41184425 & $\quad-1.17987430$ & -4.14404202 & 0.41700000 \\
\hline 0.00007331 & 0.40001352 & & \\
\hline-3.86429667 & 0.73954272 & 2.21400285 & -0.83400000 \\
\hline 0.00024239 & 3.15057423 & & \\
\hline-4.68699312 & 0.75686336 & 2.73384047 & 0.41700000 \\
\hline 0.00007331 & 0.40001352 & & \\
\hline-3.70628762 & 1.68097520 & 2.28645182 & 0.41700000 \\
\hline 0.00007331 & 0.40001352 & & \\
\hline-8.15234089 & 2.87343121 & -5.31772280 & -0.83400000 \\
\hline 0.00024239 & 3.15057423 & & \\
\hline-8.38430595 & $\quad 2.13287497$ & -4.75802040 & 0.41700000 \\
\hline 0.00007331 & 0.40001352 & & \\
\hline-8.59313869 & 3.51260829 & -4.68990803 & 0.41700000 \\
\hline 0.00007331 & 0.40001352 & & \\
\hline-13.90095207 & -1.92863603 & -2.64111324 & -0.83400000 \\
\hline 0.00024239 & 3.15057423 & & \\
\hline-14.04746172 & -2.68678375 & -3.22457357 & 0.41700000 \\
\hline 0.00007331 & 0.40001352 & & \\
\hline-14.29936955 & $\quad-1.32203813$ & -3.31445737 & 0.41700000 \\
\hline 0.00007331 & 0.40001352 & & \\
\hline-7.81349421 & -8.34208012 & 1.75187039 & -0.83400000 \\
\hline 0.00024239 & 3.15057423 & & \\
\hline-8.14758301 & -7.58741093 & 1.27109015 & 0.41700000 \\
\hline 0.00007331 & 0.40001352 & & \\
\hline-8.31437016 & -8.25825024 & 2.58634329 & 0.41700000 \\
\hline 0.00007331 & 0.40001352 & & \\
\hline-2.40375209 & -0.54637957 & -6.65046597 & -0.83400000 \\
\hline 0.00024239 & 3.15057423 & & \\
\hline-3.09874248 & -1.17887616 & -6.82425451 & 0.41700000 \\
\hline 0.00007331 & 0.40001352 & & \\
\hline-2.29158998 & -0.65922529 & -5.67838287 & 0.41700000 \\
\hline 0.00007331 & 0.40001352 & & \\
\hline-12.12910626 & 2.02758436 & 4.74833201 & -0.83400000 \\
\hline 0.00024239 & 3.15057423 & & \\
\hline-11.90799639 & 2.35200636 & 3.82883370 & 0.41700000 \\
\hline 0.00007331 & 0.40001352 & & \\
\hline-12.94300863 & 2.47341697 & 5.02678840 & 0.41700000 \\
\hline 0.00007331 & 0.40001352 & & \\
\hline-8.13464069 & 1.90512717 & 1.44615233 & -0.83400000 \\
\hline 0.00024239 & 3.15057423 & & \\
\hline-8.76016331 & 1.75818563 & 0.71094149 & 0.41700000 \\
\hline 0.00007331 & 0.40001352 & & \\
\hline-7.80995941 & 0.98576325 & 1.45561659 & 0.41700000 \\
\hline 0.00007331 & 0.40001352 & & \\
\hline 4.77623844 & $\quad 1.17368972$ & 2.88786459 & -0.83400000 \\
\hline 0.00024239 & 3.15057423 & & \\
\hline 4.95773411 & 1.47990453 & 3.83131814 & 0.41700000 \\
\hline 0.00007331 & 0.40001352 & & \\
\hline 3.92742062 & 1.64190423 & 2.80566359 & 0.41700000 \\
\hline 0.00007331 & 0.40001352 & & \\
\hline 0.74211888 & 3.98345270 & -12.58798620 & -0.83400000 \\
\hline
\end{tabular}




\begin{tabular}{|c|c|c|c|}
\hline 0.00024239 & 3.15057423 & & \\
\hline 0.60185914 & 4.04047788 & -13.53514406 & 0.41700000 \\
\hline 0.00007331 & 0.40001352 & & \\
\hline 0.97192983 & 3.06695114 & -12.54137203 & 0.41700000 \\
\hline 0.00007331 & 0.40001352 & & \\
\hline-0.76769174 & -7.02604163 & -11.16328187 & -0.83400000 \\
\hline 0.00024239 & 3.15057423 & & \\
\hline-0.38186277 & -7.78137744 & -10.63009954 & 0.41700000 \\
\hline 0.00007331 & 0.40001352 & & \\
\hline-1.22818960 & -6.46307004 & -10.47652889 & 0.41700000 \\
\hline 0.00007331 & 0.40001352 & & \\
\hline 4.37003708 & 2.76025224 & -6.92355156 & -0.83400000 \\
\hline 0.00024239 & 3.15057423 & & \\
\hline 3.92463827 & 3.15142441 & -7.70465279 & 0.41700000 \\
\hline 0.00007331 & 0.40001352 & & \\
\hline 4.50914335 & 3.59152150 & -6.37731504 & 0.41700000 \\
\hline 0.00007331 & 0.40001352 & & \\
\hline-8.30797672 & -1.39353228 & -1.91907835 & -0.83400000 \\
\hline 0.00024239 & 3.15057423 & & \\
\hline-9.26729870 & -1.47033036 & -2.11512351 & 0.41700000 \\
\hline 0.00007331 & 0.40001352 & & \\
\hline-8.23423767 & -0.78818732 & -1.15559924 & 0.41700000 \\
\hline 0.00007331 & 0.40001352 & & \\
\hline-2.28016353 & -7.50444031 & -1.97101736 & -0.83400000 \\
\hline 0.00024239 & 3.15057423 & & \\
\hline-2.13940763 & -8.43739891 & -2.08793688 & 0.41700000 \\
\hline 0.00007331 & 0.40001352 & & \\
\hline-1.92836106 & -7.39228487 & -1.08472371 & 0.41700000 \\
\hline 0.00007331 & 0.40001352 & & \\
\hline-1.29852414 & -2.00593233 & -1.39815152 & -0.83400000 \\
\hline 0.00024239 & 3.15057423 & & \\
\hline-2.06059861 & -2.63118434 & -1.22234380 & 0.41700000 \\
\hline 0.00007331 & 0.40001352 & & \\
\hline-1.35011625 & -1.50407732 & -0.53287137 & 0.41700000 \\
\hline 0.00007331 & 0.40001352 & & \\
\hline-1.25620568 & 9.07398510 & -3.14377642 & -0.83400000 \\
\hline 0.00024239 & 3.15057423 & & \\
\hline-0.98548353 & 8.96021366 & -2.24652028 & 0.41700000 \\
\hline 0.00007331 & 0.40001352 & & \\
\hline-1.43802559 & 9.99148083 & -3.28466463 & 0.41700000 \\
\hline 0.00007331 & 0.40001352 & & \\
\hline-2.59129238 & 7.64402485 & -8.00587845 & -0.83400000 \\
\hline 0.00024239 & 3.15057423 & & \\
\hline-2.31897163 & 8.41683292 & -7.51239729 & 0.41700000 \\
\hline 0.00007331 & 0.40001352 & & \\
\hline-3.05295825 & 7.16875267 & -7.28372812 & 0.41700000 \\
\hline 0.00007331 & 0.40001352 & & \\
\hline-11.90737121 & 0.19829425 & -11.09515112 & -0.83400000 \\
\hline 0.00024239 & 3.15057423 & & \\
\hline-11.97140186 & 0.54969701 & -12.00583546 & 0.41700000 \\
\hline 0.00007331 & 0.40001352 & & \\
\hline-11.10978763 & 0.66691789 & -10.84755104 & 0.41700000 \\
\hline 0.00007331 & 0.40001352 & & \\
\hline-6.82864778 & 1.72701606 & -13.89898937 & -0.83400000 \\
\hline 0.00024239 & 3.15057423 & & \\
\hline
\end{tabular}




\begin{tabular}{|c|c|c|c|}
\hline-7.48352640 & 2.39244613 & -13.71178930 & 0.41700000 \\
\hline 0.00007331 & 0.40001352 & & \\
\hline-6.28011625 & 1.74514827 & -13.09398358 & 0.41700000 \\
\hline $0.00007331 \quad 0$ & 0.40001352 & & \\
\hline 0.55798095 & -1.10797775 & -10.47128677 & -0.83400000 \\
\hline 0.00024239 & 3.15057423 & & \\
\hline-0.24944159 & -0.74474925 & -9.97930431 & 0.41700000 \\
\hline $0.00007331 \quad 0$ & 0.40001352 & & \\
\hline 0.48203656 & -2.05238032 & -10.16823483 & 0.41700000 \\
\hline 0.00007331 & 0.40001352 & & \\
\hline-6.76074375 & -5.44309247 & -12.43423335 & -0.83400000 \\
\hline 0.00024239 & 3.15057423 & & \\
\hline-6.75596727 & -6.04231561 & -11.68501709 & 0.41700000 \\
\hline 0.00007331 & 0.40001352 & & \\
\hline-5.81740344 & -5.09057487 & -12.50117249 & 0.41700000 \\
\hline 0.00007331 & 0.40001352 & & \\
\hline-4.91705132 & -5.96508503 & 3.87170434 & -0.83400000 \\
\hline 0.00024239 & 3.15057423 & & \\
\hline-5.13534880 & -6.33181906 & 4.74279737 & 0.41700000 \\
\hline 0.00007331 & 0.40001352 & & \\
\hline-5.50008392 & -5.18575287 & 3.93029785 & 0.41700000 \\
\hline $0.00007331 \quad 0$ & 0.40001352 & & \\
\hline-10.94529121 & 6.49569544 & -7.19758909 & -0.83400000 \\
\hline 0.00024239 & 3.15057423 & & \\
\hline-10.64577310 & 7.17182794 & -6.54275530 & 0.41700000 \\
\hline $0.00007331 \quad 0$ & 0.40001352 & & \\
\hline-11.34200495 & 7.16338750 & -7.77787702 & 0.41700000 \\
\hline 0.00007331 & 0.40001352 & & \\
\hline 3.16204190 & 1.88890493 & -4.03848839 & -0.83400000 \\
\hline 0.00024239 & 3.15057423 & & \\
\hline 3.97982430 & 1.96310699 & -3.55065823 & 0.41700000 \\
\hline $0.00007331 \quad 0$ & 0.40001352 & & \\
\hline 3.45212984 & 2.54667330 & -4.69871569 & 0.41700000 \\
\hline 0.00007331 & 0.40001352 & & \\
\hline-9.39131472 & 3.34740822 & 3.65268476 & -0.83400000 \\
\hline 0.00024239 & 3.15057423 & & \\
\hline-10.35637018 & 3.26801233 & 3.31626825 & 0.41700000 \\
\hline 0.00007331 & 0.40001352 & & \\
\hline-8.87785456 & 2.97510366 & 2.96392123 & 0.41700000 \\
\hline 0.00007331 & 0.40001352 & & \\
\hline 5.33299685 & -0.72178423 & 1.01310968 & -0.83400000 \\
\hline 0.00024239 & 3.15057423 & & \\
\hline 4.98679018 & 0.00726878 & 1.51931453 & 0.41700000 \\
\hline 0.00007331 & 0.40001352 & & \\
\hline 5.07496309 & -0.48181382 & 0.13991784 & 0.41700000 \\
\hline 0.00007331 & 0.40001352 & & \\
\hline-6.10084486 & -3.68351793 & -6.15237331 & -0.83400000 \\
\hline 0.00024239 & 3.15057423 & & \\
\hline-6.13695145 & -4.61886549 & -5.89592266 & 0.41700000 \\
\hline $0.00007331 \quad 0$ & 0.40001352 & & \\
\hline-5.82811546 & -3.25457215 & -5.30410385 & 0.41700000 \\
\hline 0.00007331 & 0.40001352 & & \\
\hline 0.76032954 & -3.48379612 & -0.22682017 & -0.83400000 \\
\hline 0.00024239 & 3.15057423 & & \\
\hline 0.42061335 & -2.77902484 & -0.75983793 & 0.41700000 \\
\hline
\end{tabular}




\begin{tabular}{|c|c|c|c|}
\hline 0.00007331 & 0.40001352 & & \\
\hline-0.05648462 & $2 \quad-3.70602298$ & 0.31795514 & 0.41700000 \\
\hline 0.00007331 & 0.40001352 & & \\
\hline 4.12509823 & $3 \quad 5.38506174$ & -5.62322903 & -0.83400000 \\
\hline 0.00024239 & 3.15057423 & & \\
\hline 4.29394341 & $1 \quad 5.72260857$ & -4.74928808 & 0.41700000 \\
\hline 0.00007331 & 0.40001352 & & \\
\hline 4.66634512 & $2 \quad 5.95089626$ & -6.21641684 & 0.41700000 \\
\hline 0.00007331 & 0.40001352 & & \\
\hline-2.69925857 & $\begin{array}{ll}7 & -5.80725384\end{array}$ & -9.22448254 & -0.83400000 \\
\hline 0.00024239 & 3.15057423 & & \\
\hline-3.01268291 & $1 \quad-5.70234489$ & -8.25645638 & 0.41700000 \\
\hline 0.00007331 & 0.40001352 & & \\
\hline-3.55955219 & $9 \quad-5.70203733$ & -9.68144321 & 0.41700000 \\
\hline 0.00007331 & 0.40001352 & & \\
\hline 5.03205967 & $76 \quad 6.04832220$ & -3.16290331 & -0.83400000 \\
\hline 0.00024239 & 3.15057423 & & \\
\hline 5.07969189 & 96.09124041 & -2.19273758 & 0.41700000 \\
\hline 0.00007331 & 0.40001352 & & \\
\hline 5.24470472 & $2 \quad 6.95099831$ & -3.37862182 & 0.41700000 \\
\hline 0.00007331 & 0.40001352 & & \\
\hline-9.36898136 & $5 \quad 5.41011095$ & -4.26578951 & -0.83400000 \\
\hline 0.00024239 & 3.15057423 & & \\
\hline-8.72502232 & 26.13347483 & -4.48604059 & 0.41700000 \\
\hline 0.00007331 & 0.40001352 & & \\
\hline-9.69041061 & $\quad 5.74940777$ & -3.38067007 & 0.41700000 \\
\hline 0.00007331 & 0.40001352 & & \\
\hline-11.61799833 & $3 \quad 3.08080570$ & 2.14025084 & -0.83400000 \\
\hline 0.00024239 & 3.15057423 & & \\
\hline-11.03162596 & $5 \quad 3.63134016$ & 1.63971142 & 0.41700000 \\
\hline 0.00007331 & 0.40001352 & & \\
\hline-12.49525187 & $7 \quad 3.27264903$ & 1.73668007 & 0.41700000 \\
\hline 0.00007331 & 0.40001352 & & \\
\hline-2.81103130 & 8.71794228 & -12.89315531 & -0.83400000 \\
\hline 0.00024239 & 3.15057423 & & \\
\hline-1.88338275 & 5.02276854 & -12.98277447 & 0.41700000 \\
\hline 0.00007331 & 0.40001352 & & \\
\hline-2.73918958 & $3 \quad 8.32624678$ & -11.99997732 & 0.41700000 \\
\hline 0.00007331 & 0.40001352 & & \\
\hline-11.25334570 & $\quad-2.65388866$ & 3.42953793 & -0.83400000 \\
\hline 0.00024239 & 3.15057423 & & \\
\hline-12.22408507 & $\begin{array}{ll}7 & -2.47943588\end{array}$ & 3.28228919 & 0.41700000 \\
\hline 0.00007331 & 0.40001352 & & \\
\hline-11.15817663 & $3 \quad-2.37865348$ & 4.29516773 & 0.41700000 \\
\hline 0.00007331 & 0.40001352 & & \\
\hline-14.00799530 & 7.01025053 & -9.47427433 & -0.83400000 \\
\hline 0.00024239 & 3.15057423 & & \\
\hline-14.57171910 & 7.13068714 & -8.68792837 & 0.41700000 \\
\hline 0.00007331 & 0.40001352 & & \\
\hline-14.04438238 & $3 \quad 6.06720861$ & -9.69673174 & 0.41700000 \\
\hline 0.00007331 & 0.40001352 & & \\
\hline-12.33667443 & 34.92322360 & 5.91163210 & -0.83400000 \\
\hline 0.00024239 & 3.15057423 & & \\
\hline-12.77645323 & $35 \quad 5.80331640$ & 5.76974515 & 0.41700000 \\
\hline 0.00007331 & 0.40001352 & & \\
\hline
\end{tabular}




\begin{tabular}{|c|c|c|c|}
\hline-13.07686398 & 4.25352408 & 5.70845715 & 0.41700000 \\
\hline 0.00007331 & 0.40001352 & & \\
\hline-4.50106001 & 9.82244968 & 0.20328268 & -0.83400000 \\
\hline 0.00024239 & 3.15057423 & & \\
\hline-3.83117175 & 9.63473892 & 0.85173446 & 0.41700000 \\
\hline 0.00007331 & 0.40001352 & & \\
\hline-5.16375017 & 10.24154758 & 0.76081568 & 0.41700000 \\
\hline 0.00007331 & 0.40001352 & & \\
\hline-2.04785728 & 5.68396378 & -10.46375847 & -0.83400000 \\
\hline 0.00024239 & 3.15057423 & & \\
\hline-2.31726146 & 4.81767797 & -10.26839066 & 0.41700000 \\
\hline 0.00007331 & 0.40001352 & & \\
\hline-2.11540723 & 6.24663305 & -9.61080551 & 0.41700000 \\
\hline 0.00007331 & 0.40001352 & & \\
\hline-15.56483099 & 3.95638359 & -3.07604356 & -0.83400000 \\
\hline 0.00024239 & 3.15057423 & & \\
\hline-15.40316412 & 4.89086066 & -3.05194088 & 0.41700000 \\
\hline 0.00007331 & 0.40001352 & & \\
\hline-15.20639679 & 3.72684411 & -3.95270773 & 0.41700000 \\
\hline 0.00007331 & 0.40001352 & & \\
\hline 1.06297350 & -0.06558885 & -0.39115554 & -0.83400000 \\
\hline 0.00024239 & 3.15057423 & & \\
\hline 0.63488048 & 0.47885665 & -1.04606795 & 0.41700000 \\
\hline 0.00007331 & 0.40001352 & & \\
\hline 0.32820570 & -0.27375838 & 0.18539919 & 0.41700000 \\
\hline 0.00007331 & 0.40001352 & & \\
\hline-3.86939263 & -2.79157066 & -7.23537540 & -0.83400000 \\
\hline 0.00024239 & 3.15057423 & & \\
\hline-3.52083158 & -3.67018366 & -7.43383360 & 0.41700000 \\
\hline 0.00007331 & 0.40001352 & & \\
\hline-4.79131699 & -3.02509856 & -7.00481272 & 0.41700000 \\
\hline 0.00007331 & 0.40001352 & & \\
\hline 3.49416780 & 1.04973090 & -0.55044836 & -0.83400000 \\
\hline 0.00024239 & 3.15057423 & & \\
\hline 3.15805387 & 1.95264065 & -0.61162758 & 0.41700000 \\
\hline 0.00007331 & 0.40001352 & & \\
\hline 2.66715837 & 0.52932620 & -0.47689819 & 0.41700000 \\
\hline 0.00007331 & 0.40001352 & & \\
\hline-2.26489282 & 9.15015507 & 1.43185747 & -0.83400000 \\
\hline 0.00024239 & 3.15057423 & & \\
\hline-1.52316463 & 9.17018604 & 0.77595973 & 0.41700000 \\
\hline 0.00007331 & 0.40001352 & & \\
\hline-2.15654683 & 10.03418350 & 1.85911751 & 0.41700000 \\
\hline 0.00007331 & 0.40001352 & & \\
\hline-7.63225603 & -0.85161811 & 1.89244413 & -0.83400000 \\
\hline 0.00024239 & 3.15057423 & & \\
\hline-7.57100630 & -1.26256263 & 2.78277183 & 0.41700000 \\
\hline 0.00007331 & 0.40001352 & & \\
\hline-8.24349785 & $\quad-1.45958865$ & 1.43706441 & 0.41700000 \\
\hline 0.00007331 & 0.40001352 & & \\
\hline 4.56507969 & 8.72389984 & -4.05687284 & -0.83400000 \\
\hline 0.00024239 & 3.15057423 & & \\
\hline 5.49787426 & $\quad 8.77483273$ & -4.12891150 & 0.41700000 \\
\hline 0.00007331 & 0.40001352 & & \\
\hline 4.51406193 & 9.59997559 & -3.65144944 & 0.41700000 \\
\hline
\end{tabular}




\begin{tabular}{|c|c|c|c|}
\hline 0.00007331 & 0.40001352 & & \\
\hline-9.34895897 & 6.58639002 & 2.19171357 & -0.83400000 \\
\hline 0.00024239 & 3.15057423 & & \\
\hline-9.33700466 & 6.57118511 & 3.15167809 & 0.41700000 \\
\hline 0.00007331 & 0.40001352 & & \\
\hline-8.59110069 & 6.00167227 & 1.94855523 & 0.41700000 \\
\hline 0.00007331 & 0.40001352 & & \\
\hline-9.33462048 & 1.23601043 & -0.92071909 & -0.83400000 \\
\hline 0.00024239 & 3.15057423 & & \\
\hline-10.27926826 & 1.31667566 & -1.02176487 & 0.41700000 \\
\hline 0.00007331 & 0.40001352 & & \\
\hline-9.16207790 & 1.35826623 & -1.84440970 & 0.41700000 \\
\hline 0.00007331 & 0.40001352 & & \\
\hline-1.01151693 & -3.85261488 & -4.38280582 & -0.83400000 \\
\hline 0.00024239 & 3.15057423 & & \\
\hline-0.47555986 & -3.88193035 & -5.18132019 & 0.41700000 \\
\hline 0.00007331 & 0.40001352 & & \\
\hline-1.17485571 & -2.93691993 & -4.36469316 & 0.41700000 \\
\hline 0.00007331 & 0.40001352 & & \\
\hline 2.05352044 & 5.26270914 & -3.81057954 & -0.83400000 \\
\hline 0.00024239 & 3.15057423 & & \\
\hline 2.30209565 & 5.01257896 & -4.75035810 & 0.41700000 \\
\hline 0.00007331 & 0.40001352 & & \\
\hline 2.00577831 & 6.25401068 & -3.82367873 & 0.41700000 \\
\hline 0.00007331 & 0.40001352 & & \\
\hline-5.97430611 & -3.35284114 & 3.66271663 & -0.83400000 \\
\hline 0.00024239 & 3.15057423 & & \\
\hline-6.34943104 & $=-3.33122945$ & 2.73790097 & 0.41700000 \\
\hline 0.00007331 & 0.40001352 & & \\
\hline-6.79119682 & -3.41774416 & 4.22118616 & 0.41700000 \\
\hline 0.00007331 & 0.40001352 & & \\
\hline-4.07392979 & -5.21918535 & 1.08563566 & -0.83400000 \\
\hline 0.00024239 & 3.15057423 & & \\
\hline-3.44744134 & $=-5.98561859$ & 1.17205524 & 0.41700000 \\
\hline 0.00007331 & 0.40001352 & & \\
\hline-4.25162745 & -5.11320877 & 2.05839086 & 0.41700000 \\
\hline 0.00007331 & 0.40001352 & & \\
\hline-2.21267319 & -6.27845049 & -4.61125135 & -0.83400000 \\
\hline 0.00024239 & 3.15057423 & & \\
\hline-1.91711199 & -5.39501381 & -4.35645103 & 0.41700000 \\
\hline 0.00007331 & 0.40001352 & & \\
\hline-2.53220248 & -6.62919712 & -3.75691271 & 0.41700000 \\
\hline 0.00007331 & 0.40001352 & & \\
\hline-9.53280926 & -4.88076448 & 3.80372620 & -0.83400000 \\
\hline 0.00024239 & 3.15057423 & & \\
\hline-8.99044991 & -4.07709312 & 3.85303903 & 0.41700000 \\
\hline 0.00007331 & 0.40001352 & & \\
\hline-10.20314503 & -4.51203346 & 3.21993613 & 0.41700000 \\
\hline 0.00007331 & 0.40001352 & & \\
\hline-8.07159270 & -7.82660449 & -6.74893344 & -0.83400000 \\
\hline 0.00024239 & 3.15057423 & & \\
\hline-8.77082718 & -7.65391792 & -7.43743528 & 0.41700000 \\
\hline 0.00007331 & 0.40001352 & & \\
\hline-7.36448348 & -7.98657335 & -7.31359351 & 0.41700000 \\
\hline 0.00007331 & 0.40001352 & & \\
\hline
\end{tabular}




\begin{tabular}{|c|c|c|c|}
\hline-11.19498127 & 5.76199588 & -12.77836721 & -0.83400000 \\
\hline 0.00024239 & 3.15057423 & & \\
\hline-10.77039545 & 6.31348988 & -12.11654918 & 0.41700000 \\
\hline 0.00007331 & 0.40001352 & & \\
\hline-11.05010145 & $\quad 6.19977089$ & -13.65626829 & 0.41700000 \\
\hline 0.00007331 & 0.40001352 & & \\
\hline-3.46119308 & 5.24027777 & -1.12712348 & -0.83400000 \\
\hline 0.00024239 & 3.15057423 & & \\
\hline-3.91522670 & 6.10867834 & -1.21982348 & 0.41700000 \\
\hline 0.00007331 & 0.40001352 & & \\
\hline-3.42272019 & 4.99315119 & -2.04138613 & 0.41700000 \\
\hline 0.00007331 & 0.40001352 & & \\
\hline 3.24086380 & -3.51059532 & -3.72262669 & -0.83400000 \\
\hline 0.00024239 & 3.15057423 & & \\
\hline 2.88096380 & -2.71320820 & -3.18550754 & 0.41700000 \\
\hline 0.00007331 & 0.40001352 & & \\
\hline 3.92413592 & -3.01082754 & -4.34126472 & 0.41700000 \\
\hline 0.00007331 & 0.40001352 & & \\
\hline 1.75788546 & $\quad 0.10917673$ & 4.03318119 & -0.83400000 \\
\hline 0.00024239 & 3.15057423 & & \\
\hline 1.86394334 & $\quad-0.17879789$ & 4.93115330 & 0.41700000 \\
\hline 0.00007331 & 0.40001352 & & \\
\hline 1.20584977 & -0.65019161 & 3.76357555 & 0.41700000 \\
\hline 0.00007331 & 0.40001352 & & \\
\hline-4.31579109 & 1.46899585 & -12.09816191 & -0.83400000 \\
\hline 0.00024239 & 3.15057423 & & \\
\hline-5.03805347 & 1.03099184 & -11.60303042 & 0.41700000 \\
\hline 0.00007331 & 0.40001352 & & \\
\hline-4.27556987 & 2.34382228 & -11.59347937 & 0.41700000 \\
\hline 0.00007331 & 0.40001352 & & \\
\hline-8.67339897 & -5.38950539 & -3.40987897 & -0.83400000 \\
\hline 0.00024239 & 3.15057423 & & \\
\hline-8.08180046 & -5.60862160 & -4.11813736 & 0.41700000 \\
\hline 0.00007331 & 0.40001352 & & \\
\hline-8.06923866 & $\quad-4.93459272$ & -2.73378325 & 0.41700000 \\
\hline 0.00007331 & 0.40001352 & & \\
\hline-4.98947447 & 6.34384319 & -11.47277468 & -0.83400000 \\
\hline 0.00024239 & 3.15057423 & & \\
\hline-4.38051813 & 6.99816831 & -11.06174009 & 0.41700000 \\
\hline 0.00007331 & 0.40001352 & & \\
\hline-5.62951296 & 6.29497203 & -10.76720922 & 0.41700000 \\
\hline 0.00007331 & 0.40001352 & & \\
\hline-8.27862644 & $\quad-6.22921848$ & 0.11456603 & -0.83400000 \\
\hline 0.00024239 & 3.15057423 & & \\
\hline-9.20361233 & -5.98716402 & 0.10752918 & 0.41700000 \\
\hline 0.00007331 & 0.40001352 & & \\
\hline-8.23515320 & -6.65712786 & -0.77678341 & 0.41700000 \\
\hline 0.00007331 & 0.40001352 & & \\
\hline-12.20935461 & 7.45031266 & 4.53264741 & -0.83400000 \\
\hline 0.00024239 & 3.15057423 & & \\
\hline-11.38798830 & 6.89944844 & 4.40146951 & 0.41700000 \\
\hline 0.00007331 & 0.40001352 & & \\
\hline-12.55618403 & 7.59442238 & 3.63910709 & 0.41700000 \\
\hline 0.00007331 & 0.40001352 & & \\
\hline-6.23802818 & -2.05695356 & -12.25023545 & -0.83400000 \\
\hline
\end{tabular}




\begin{tabular}{|c|c|c|c|}
\hline 0.00024239 & 3.15057423 & & \\
\hline-6.13116277 & -2.92388261 & -11.77491231 & 0.41700000 \\
\hline 0.00007331 & 0.40001352 & & \\
\hline-5.61272014 & -2.27334703 & -12.91469634 & 0.41700000 \\
\hline 0.00007331 & 0.40001352 & & \\
\hline-6.09605598 & 3.33998847 & 4.70277500 & -0.83400000 \\
\hline 0.00024239 & 3.15057423 & & \\
\hline-6.38206244 & 4.09658241 & 5.27146816 & 0.41700000 \\
\hline 0.00007331 & 0.40001352 & & \\
\hline-5.20117903 & 3.37160611 & 4.91095448 & 0.41700000 \\
\hline 0.00007331 & 0.40001352 & & \\
\hline-5.52061605 & 7.17474270 & 2.26865196 & -0.83400000 \\
\hline 0.00024239 & 3.15057423 & & \\
\hline-6.20627928 & 6.43898106 & 2.35506892 & 0.41700000 \\
\hline 0.00007331 & 0.40001352 & & \\
\hline-4.70384932 & 6.67641544 & 2.00695515 & 0.41700000 \\
\hline 0.00007331 & 0.40001352 & & \\
\hline-13.65620129 & 7.45130690 & -5.26471823 & -0.83400000 \\
\hline 0.00024239 & 3.15057423 & & \\
\hline-14.23529415 & 7.70012941 & -5.97015684 & 0.41700000 \\
\hline 0.00007331 & 0.40001352 & & \\
\hline-12.97290819 & 8.15358284 & -5.38182562 & 0.41700000 \\
\hline 0.00007331 & 0.40001352 & & \\
\hline-0.15509634 & -2.72927952 & 6.89051485 & -0.83400000 \\
\hline 0.00024239 & 3.15057423 & & \\
\hline-0.82812691 & -3.00526023 & 7.53619385 & 0.41700000 \\
\hline 0.00007331 & 0.40001352 & & \\
\hline-0.73481238 & -2.54001141 & 6.12987900 & 0.41700000 \\
\hline 0.00007331 & 0.40001352 & & \\
\hline-12.08586591 & 9.46859130 & -6.14232176 & -0.83400000 \\
\hline 0.00024239 & 3.15057423 & & \\
\hline-12.37590473 & 10.00505051 & -6.89231603 & 0.41700000 \\
\hline 0.00007331 & 0.40001352 & & \\
\hline-11.43954294 & $=9.98039016$ & -5.61479585 & 0.41700000 \\
\hline 0.00007331 & 0.40001352 & & \\
\hline-13.77178452 & -1.77177949 & 3.01155768 & -0.83400000 \\
\hline 0.00024239 & 3.15057423 & & \\
\hline-13.99905369 & -1.46797081 & 3.87259972 & 0.41700000 \\
\hline 0.00007331 & 0.40001352 & & \\
\hline-13.96769497 & -0.96654649 & 2.47607974 & 0.41700000 \\
\hline 0.00007331 & 0.40001352 & & \\
\hline-9.07558553 & 3.02668914 & -12.63456457 & -0.83400000 \\
\hline 0.00024239 & 3.15057423 & & \\
\hline-9.34321707 & 3.15119704 & -13.57319062 & 0.41700000 \\
\hline 0.00007331 & 0.40001352 & & \\
\hline-8.43977182 & 3.74490556 & -12.58055227 & 0.41700000 \\
\hline 0.00007331 & 0.40001352 & & \\
\hline-12.64775536 & 9.15957074 & -2.72061105 & -0.83400000 \\
\hline 0.00024239 & 3.15057423 & & \\
\hline-12.22316954 & $\quad 10.00026898$ & -2.50208421 & 0.41700000 \\
\hline 0.00007331 & 0.40001352 & & \\
\hline-13.41526100 & 9.49654822 & -3.12602325 & 0.41700000 \\
\hline 0.00007331 & 0.40001352 & & \\
\hline-14.14414046 & 6.12802414 & 0.60974650 & -0.83400000 \\
\hline 0.00024239 & 3.15057423 & & \\
\hline
\end{tabular}




\begin{tabular}{|c|c|c|c|}
\hline-13.50532505 & 5.49088292 & 0.29932980 & 0.41700000 \\
\hline 0.00007331 & 0.40001352 & & \\
\hline-14.30748865 & 5.77580695 & 1.50289587 & 0.41700000 \\
\hline 0.00007331 & 0.40001352 & & \\
\hline-3.58556791 & 4.65147798 & -13.03767273 & -0.83400000 \\
\hline 0.00024239 & 3.15057423 & & \\
\hline-2.69634958 & 5.00402955 & -12.93018744 & 0.41700000 \\
\hline 0.00007331 & 0.40001352 & & \\
\hline-4.19563766 & 5.29573004 & -12.55840990 & 0.41700000 \\
\hline 0.00007331 & 0.40001352 & & \\
\hline-12.75514815 & 3.79680629 & -0.63400074 & -0.83400000 \\
\hline 0.00024239 & 3.15057423 & & \\
\hline-12.09488985 & 4.44156812 & -0.37973495 & 0.41700000 \\
\hline 0.00007331 & 0.40001352 & & \\
\hline-12.15647814 & 3.19973974 & -1.14255233 & 0.41700000 \\
\hline 0.00007331 & 0.40001352 & & \\
\hline-2.82846212 & 5.86562014 & 4.46032476 & -0.83400000 \\
\hline 0.00024239 & 3.15057423 & & \\
\hline-1.94455612 & 5.60928631 & 4.17307854 & 0.41700000 \\
\hline 0.00007331 & 0.40001352 & & \\
\hline-2.69034266 & 6.77059746 & 4.29284573 & 0.41700000 \\
\hline 0.00007331 & 0.40001352 & & \\
\hline-0.74459024 & -1.47454067 & -13.34422085 & -0.83400000 \\
\hline 0.00024239 & 3.15057423 & & \\
\hline-0.96498151 & -0.51782318 & -13.23167774 & 0.41700000 \\
\hline 0.00007331 & 0.40001352 & & \\
\hline-1.36986490 & -1.90669199 & -12.73271248 & 0.41700000 \\
\hline 0.00007331 & 0.40001352 & & \\
\hline-11.42049568 & 1.74410542 & -6.20227831 & -0.83400000 \\
\hline 0.00024239 & 3.15057423 & & \\
\hline-11.45701950 & 2.53933820 & -5.56972616 & 0.41700000 \\
\hline 0.00007331 & 0.40001352 & & \\
\hline-11.59960716 & 2.22951516 & -7.01957052 & 0.41700000 \\
\hline 0.00007331 & 0.40001352 & & \\
\hline 2.00765657 & -5.96039009 & -4.22972727 & -0.83400000 \\
\hline 0.00024239 & 3.15057423 & & \\
\hline 1.66777205 & -5.67990160 & -3.34176111 & 0.41700000 \\
\hline 0.00007331 & 0.40001352 & & \\
\hline 2.59278393 & -5.23373747 & -4.40355873 & 0.41700000 \\
\hline 0.00007331 & 0.40001352 & & \\
\hline-1.80957465 & 13.41002819 & -1.08127956 & -0.83400000 \\
\hline 0.00024239 & 3.15057423 & & \\
\hline-2.67182962 & 12.98799917 & -1.07766084 & 0.41700000 \\
\hline 0.00007331 & 0.40001352 & & \\
\hline-2.11009364 & 14.30828163 & -0.96596484 & 0.41700000 \\
\hline 0.00007331 & 0.40001352 & & \\
\hline 3.06266475 & -2.00935292 & -8.57498169 & -0.83400000 \\
\hline 0.00024239 & 3.15057423 & & \\
\hline 2.29461861 & -2.45595694 & -8.22233963 & 0.41700000 \\
\hline 0.00007331 & 0.40001352 & & \\
\hline 3.88176322 & -2.37163520 & -8.21736526 & 0.41700000 \\
\hline 0.00007331 & 0.40001352 & & \\
\hline 5.41639662 & 6.98136330 & -7.56164074 & -0.83400000 \\
\hline 0.00024239 & 3.15057423 & & \\
\hline 4.51864910 & 7.33842087 & -7.54427862 & 0.41700000 \\
\hline
\end{tabular}




\begin{tabular}{|c|c|c|c|}
\hline 0.00007331 & 0.40001352 & & \\
\hline 5.68595123 & 7.35090637 & -8.42472267 & 0.41700000 \\
\hline 0.00007331 & 0.40001352 & & \\
\hline-12.95901034 & 5.69611732 & -3.22328277 & -0.83400000 \\
\hline 0.00024239 & 3.15057423 & & \\
\hline-13.77139923 & 5.68432896 & -2.69825788 & 0.41700000 \\
\hline 0.00007331 & 0.40001352 & & \\
\hline-13.34648249 & 6.33859949 & -3.87178178 & 0.41700000 \\
\hline 0.00007331 & 0.40001352 & & \\
\hline 2.86162949 & 4.83362484 & 1.04894245 & -0.83400000 \\
\hline 0.00024239 & 3.15057423 & & \\
\hline 2.94785142 & 4.43282461 & 0.15239438 & 0.41700000 \\
\hline 0.00007331 & 0.40001352 & & \\
\hline 2.96798277 & 4.03178787 & 1.60738480 & 0.41700000 \\
\hline 0.00007331 & 0.40001352 & & \\
\hline-0.37394977 & 3.92270803 & 2.80023980 & -0.83400000 \\
\hline 0.00024239 & 3.15057423 & & \\
\hline-0.68419307 & 3.90205503 & 1.88045883 & 0.41700000 \\
\hline 0.00007331 & 0.40001352 & & \\
\hline-0.54673797 & 3.04112530 & 3.16742992 & 0.41700000 \\
\hline 0.00007331 & 0.40001352 & & \\
\hline-1.33274293 & -1.08798683 & -4.14268970 & -0.83400000 \\
\hline 0.00024239 & 3.15057423 & & \\
\hline-0.58509195 & -0.49760401 & -4.26913738 & 0.41700000 \\
\hline 0.00007331 & 0.40001352 & & \\
\hline-1.57219243 & -0.88222152 & -3.26186395 & 0.41700000 \\
\hline 0.00007331 & 0.40001352 & & \\
\hline 0.51386356 & -4.55409622 & 4.08526278 & -0.83400000 \\
\hline 0.00024239 & 3.15057423 & & \\
\hline 0.54449826 & -5.05407763 & 4.86631298 & 0.41700000 \\
\hline 0.00007331 & 0.40001352 & & \\
\hline 1.17675662 & -3.82966375 & 4.26508665 & 0.41700000 \\
\hline 0.00007331 & 0.40001352 & & \\
\hline-9.03044796 & 0.01655403 & -7.12107897 & -0.83400000 \\
\hline 0.00024239 & 3.15057423 & & \\
\hline-8.20575523 & -0.06905016 & -6.59003067 & 0.41700000 \\
\hline 0.00007331 & 0.40001352 & & \\
\hline-9.60659695 & 0.42270315 & -6.47083044 & 0.41700000 \\
\hline 0.00007331 & 0.40001352 & & \\
\hline-5.23969854 & $=-8.46711982$ & -8.28344024 & -0.83400000 \\
\hline 0.00024239 & 3.15057423 & & \\
\hline-5.52945674 & $=-9.31833971$ & -7.88173354 & 0.41700000 \\
\hline 0.00007331 & 0.40001352 & & \\
\hline-4.30740798 & -8.55647839 & -8.23987974 & 0.41700000 \\
\hline 0.00007331 & 0.40001352 & & \\
\hline 1.25795798 & 1.03646688 & -12.03802130 & -0.83400000 \\
\hline 0.00024239 & 3.15057423 & & \\
\hline 0.44199638 & 0.96703128 & -12.53276369 & 0.41700000 \\
\hline 0.00007331 & 0.40001352 & & \\
\hline 1.25303463 & 0.28819088 & -11.38923762 & 0.41700000 \\
\hline 0.00007331 & 0.40001352 & & \\
\hline-3.86322170 & 8.42595885 & -10.16992955 & -0.83400000 \\
\hline 0.00024239 & 3.15057423 & & \\
\hline-4.20128625 & 9.35292456 & -10.00118308 & 0.41700000 \\
\hline 0.00007331 & 0.40001352 & & \\
\hline
\end{tabular}




\begin{tabular}{|c|c|c|c|}
\hline-3.28508107 & 8.33410094 & -9.41658883 & 0.41700000 \\
\hline 0.00007331 & 0.40001352 & & \\
\hline-1.39618202 & 1.11947588 & -12.98610136 & -0.83400000 \\
\hline 0.00024239 & 3.15057423 & & \\
\hline-1.43460079 & 2.01820723 & -13.32191441 & 0.41700000 \\
\hline 0.00007331 & 0.40001352 & & \\
\hline-2.25057884 & $\quad 1.17017154$ & -12.53048346 & 0.41700000 \\
\hline 0.00007331 & 0.40001352 & & \\
\hline-11.98060472 & -4.45980895 & -5.87099279 & -0.83400000 \\
\hline 0.00024239 & 3.15057423 & & \\
\hline-12.82667083 & -4.14411701 & -6.23088182 & 0.41700000 \\
\hline 0.00007331 & 0.40001352 & & \\
\hline-11.49747241 & -4.45754302 & -6.66067851 & 0.41700000 \\
\hline 0.00007331 & 0.40001352 & & \\
\hline-15.00323031 & 2.41336863 & -0.69267030 & -0.83400000 \\
\hline 0.00024239 & 3.15057423 & & \\
\hline-14.16930363 & 2.92504148 & -0.74159809 & 0.41700000 \\
\hline 0.00007331 & 0.40001352 & & \\
\hline-15.41205666 & $\quad 2.73811428$ & -1.49574371 & 0.41700000 \\
\hline 0.00007331 & 0.40001352 & & \\
\hline-6.21218586 & 5.15519667 & -4.94121981 & -0.83400000 \\
\hline 0.00024239 & 3.15057423 & & \\
\hline-6.72395086 & 4.43384933 & -5.30777979 & 0.41700000 \\
\hline 0.00007331 & 0.40001352 & & \\
\hline-5.42491817 & 4.68438816 & -4.57948351 & 0.41700000 \\
\hline 0.00007331 & 0.40001352 & & \\
\hline-2.29626624 & $\quad 11.47527525$ & -9.14929299 & -0.83400000 \\
\hline 0.00024239 & 3.15057423 & & \\
\hline-2.29822628 & 12.39635966 & -8.84270148 & 0.41700000 \\
\hline 0.00007331 & 0.40001352 & & \\
\hline-3.22399269 & $\quad 11.25381300$ & -9.08112816 & 0.41700000 \\
\hline 0.00007331 & 0.40001352 & & \\
\hline-5.04480140 & 11.05734179 & -9.18205981 & -0.83400000 \\
\hline 0.00024239 & 3.15057423 & & \\
\hline-5.45388369 & 11.94699976 & -9.04231505 & 0.41700000 \\
\hline 0.00007331 & 0.40001352 & & \\
\hline-5.47470669 & 10.61978934 & -8.38914208 & 0.41700000 \\
\hline 0.00007331 & 0.40001352 & & \\
\hline-12.99631064 & $\quad 4.39289984$ & -11.04582184 & -0.83400000 \\
\hline 0.00024239 & 3.15057423 & & \\
\hline-12.52935117 & 5.03446301 & -11.59578340 & 0.41700000 \\
\hline 0.00007331 & 0.40001352 & & \\
\hline-12.39318173 & 3.59869632 & -11.06841629 & 0.41700000 \\
\hline 0.00007331 & 0.40001352 & & \\
\hline-14.18057526 & -3.40689767 & -7.67142881 & -0.83400000 \\
\hline 0.00024239 & 3.15057423 & & \\
\hline-14.31170643 & -3.11911500 & -8.59677041 & 0.41700000 \\
\hline 0.00007331 & 0.40001352 & & \\
\hline-14.29993022 & -2.60616792 & -7.08094276 & 0.41700000 \\
\hline 0.00007331 & 0.40001352 & & \\
\hline 1.48241453 & -3.17238231 & -13.02713272 & -0.83400000 \\
\hline 0.00024239 & 3.15057423 & & \\
\hline 0.96281199 & -2.33442588 & -12.99476597 & 0.41700000 \\
\hline 0.00007331 & 0.40001352 & & \\
\hline 0.82027153 & -3.64223047 & -13.57238743 & 0.41700000 \\
\hline
\end{tabular}




\begin{tabular}{|c|c|c|c|c|}
\hline \multicolumn{4}{|c|}{0.40001352} & \$end \\
\hline \multicolumn{5}{|c|}{$\$ 1 j$ parameters } \\
\hline 1 & 0.00003187 & & 36 & \\
\hline 2 & 0.00019123 & & & \\
\hline 3 & 0.00009562 & & & \\
\hline 4 & 0.00005578 & & 356 & \\
\hline 5 & 0.00005578 & & & \\
\hline 6 & 0.00009562 & & 82 & \\
\hline 7 & 0.00005578 & & 356 & \\
\hline 8 & 0.00005578 & & 356 & \\
\hline 9 & 0.00003187 & & & \\
\hline 10 & 0.00019123 & & 64 & \\
\hline 11 & 0.00009562 & & & \\
\hline 12 & 0.00005578 & & & \\
\hline 13 & 0.00005578 & & & \\
\hline 14 & 0.00007331 & & & \\
\hline 15 & 0.00030613 & & & \\
\hline 16 & 0.00054661 & & 10 & \\
\hline 17 & 0.00024239 & & 23 & \\
\hline 18 & 0.00007331 & & & \\
\hline 19 & 0.00007331 & & & \\
\hline \multicolumn{5}{|c|}{ \$end } \\
\hline \multicolumn{5}{|c|}{$\begin{array}{l}\text { \$comment } \\
\text { qchem control file needed for the } \\
\text { scan.inp } \\
\text { \$end }\end{array}$} \\
\hline \multicolumn{5}{|c|}{ \$rem } \\
\hline \multirow{2}{*}{\multicolumn{2}{|c|}{$\begin{array}{l}\text { igdesp } \\
\text { symmetry }\end{array}$}} & \\
\hline & & \multirow{2}{*}{\multicolumn{2}{|c|}{$\begin{array}{l}\text { off } \\
\text { true }\end{array}$}} & \\
\hline \multicolumn{2}{|c|}{ sym ignore } & \multirow{2}{*}{\multicolumn{2}{|c|}{ true }} & \\
\hline \multicolumn{2}{|c|}{ exchange } & & & \\
\hline \multicolumn{2}{|c|}{ basis } & \multicolumn{2}{|c|}{$6-31+9 * *$} & \\
\hline \multicolumn{2}{|c|}{ qm_mm } & \multicolumn{2}{|c|}{ true } & \\
\hline \multicolumn{2}{|c|}{ job̄type } & \multicolumn{2}{|c|}{ force } & \\
\hline \multicolumn{2}{|c|}{ symmetry } & \multicolumn{2}{|c|}{ off } & \\
\hline \multicolumn{2}{|c|}{ sym_ignore } & & \\
\hline \multicolumn{2}{|c|}{ print_input } & false & & \\
\hline qmmn & & true & & \\
\hline $\operatorname{scf}$ & rithm & diis_ & & \\
\hline $\operatorname{scf}^{-}$ & s_always & true & & \\
\hline thre & & 14 & & \\
\hline $\operatorname{scf}$ & ergence & 9 & & \\
\hline $\begin{array}{l}\max \\
\text { \$enc }\end{array}$ & cycles & 200 & & \\
\hline \$mol & & & & \\
\hline 01 & & & & \\
\hline $\mathrm{C}$ & -4.5302 & 29979 & 0.3474980463 & -3.4482620388 \\
\hline 0 & -4.2821 & 42065 & -0.6443653521 & -4.1159852232 \\
\hline $\mathrm{C}$ & -4.2589 & 33280 & 0.4728237024 & -1.9548600563 \\
\hline $\mathrm{H}$ & -3.1853 & 44153 & 0.4014444707 & -1.7546327052 \\
\hline
\end{tabular}




\begin{tabular}{|cccc|}
\hline $\mathrm{H}$ & -4.7318365026 & -0.3880481106 & -1.4758713928 \\
$\mathrm{C}$ & -4.8803182970 & 1.8151120642 & -1.5142897172 \\
$\mathrm{H}$ & -4.1078092431 & 2.5797624642 & -1.3630062200 \\
$\mathrm{H}$ & -5.4671954870 & 1.7724772590 & -0.5921306735 \\
$\mathrm{C}$ & -5.7213707362 & 2.2742501489 & -2.6949769955 \\
$\mathrm{O}$ & -6.6676775358 & 3.0452749043 & -2.6486124046 \\
$\mathrm{C}$ & -5.2295935503 & 1.5944700901 & -3.9544916891 \\
$\mathrm{H}$ & -4.4735430443 & 2.2400199363 & -4.4323231722 \\
$\mathrm{H}$ & -5.9951562143 & 1.4005382003 & -4.7094519761 \\
$\mathrm{H}$ & -7.6213871300 & 3.9841879802 & -1.5545514805 \\
$\mathrm{O}$ & -8.1913255525 & 4.6144490141 & -1.0423151450 \\
$\mathrm{CL}$ & -7.3445458593 & 6.1039254073 & -1.2278823717 \\
$\mathrm{O}$ & -5.9399657407 & -0.4077018344 & -6.4716743463 \\
$\mathrm{H}$ & -5.1471612627 & -0.5542510367 & -5.9306930634 \\
$\mathrm{H}$ & -6.6122922685 & -0.9632560473 & -6.0326838892 \\
\$end & & & \\
& & & \\
& & & \\
\hline
\end{tabular}

Table 49: Script generated by CHARMM to be run by QChem for each replica in Replica Path method and CI-NEB method 
Appendix B: Topology and parameter files

B1 Topology and parameter files for sugar moleclues.

\begin{tabular}{|c|c|c|c|c|c|c|c|c|c|c|c|c|}
\hline \multirow{2}{*}{$\begin{array}{l}\text { RESI } \\
\text { GROU }\end{array}$} & AXYS & \multicolumn{4}{|c|}{0.000} & \multicolumn{7}{|c|}{ ! alpha-D-xylopyranos monomer } \\
\hline & & & & & & & & & & & & \\
\hline ATOM & $\mathrm{C} 1$ & \multicolumn{2}{|c|}{$\mathrm{CC} 3162$} & \multicolumn{2}{|c|}{0.340} & & & & & & & \\
\hline ATOM & $\mathrm{H} 1$ & \multicolumn{2}{|c|}{ HCA1 } & \multicolumn{2}{|c|}{0.090} & & & & & & & \\
\hline ATOM & O1 & \multicolumn{2}{|c|}{ OC311 } & \multicolumn{2}{|c|}{-0.650} & & & & & & & \\
\hline ATOM & $\mathrm{HO}$ & \multicolumn{2}{|c|}{$\mathrm{HCP} 1$} & \multicolumn{2}{|c|}{0.420} & & & & & & & \\
\hline ATOM & $\mathrm{C} 5$ & \multicolumn{2}{|c|}{ CC3263 } & \multicolumn{2}{|c|}{0.020} & & & & & & & \\
\hline ATOM & H51 & \multicolumn{2}{|c|}{ HCA2 } & \multicolumn{2}{|c|}{0.090} & & & & & & & \\
\hline ATOM & H52 & \multicolumn{2}{|c|}{$\mathrm{HCA} 2$} & \multicolumn{2}{|c|}{0.090} & & & & & & & \\
\hline ATOM & 05 & \multicolumn{2}{|c|}{ OC3C61 } & \multirow{2}{*}{\multicolumn{2}{|c|}{-0.400}} & & & & & & & \\
\hline \multicolumn{11}{|c|}{ GROU } & & \\
\hline ATOM & $\mathrm{C} 2$ & $\mathrm{CC} 3$ & 161 & 0 . & 140 & & & & & & & \\
\hline ATOM & $\mathrm{H} 2$ & $\mathrm{HCA}$ & & 0 . & 090 & & & & & & & \\
\hline ATOM & $\mathrm{O} 2$ & $\mathrm{OC} 3$ & & -0 . & 650 & & & & & & & \\
\hline ATOM & $\mathrm{HO} 2$ & $\mathrm{HCP}$ & & 0 . & 420 & & & & & & & \\
\hline GROU & & & & & & & & & & & & \\
\hline ATOM & C3 & $\mathrm{CC} 3$ & 161 & 0 . & 140 & & & & & & & \\
\hline ATOM & H3 & $\mathrm{HCA}$ & & 0 . & 090 & & & & & & & \\
\hline ATOM & 03 & $\mathrm{OC} 3$ & & -0 . & 650 & & & & & & & \\
\hline ATOM & $\mathrm{HO} 3$ & $\mathrm{HCP}$ & & 0 & 420 & & & & & & & \\
\hline GROU & & & & & & & & & & & & \\
\hline ATOM & $\mathrm{C} 4$ & $\mathrm{CC} 3$ & 161 & 0 . & 140 & & & & & & & \\
\hline ATOM & $\mathrm{H} 4$ & $\mathrm{HCA}$ & & 0 . & 090 & & & & & & & \\
\hline ATOM & O4 & OC3. & & -0 . & 650 & & & & & & & \\
\hline ATOM & $\mathrm{HO} 4$ & $\mathrm{HCP}$ & & 0 & 420 & & & & & & & \\
\hline BOND & $\mathrm{Cl}$ & O1 & & $\mathrm{C} 1$ & $\mathrm{H} 1$ & $\mathrm{O} 1$ & $\mathrm{HO} 1$ & $\mathrm{C} 1$ & O & & $\mathrm{C} 1$ & $\mathrm{C} 2$ \\
\hline BOND & $\mathrm{C} 2$ & $\mathrm{H} 2$ & & $\mathrm{C} 2$ & $\mathrm{O} 2$ & $\mathrm{O} 2$ & $\mathrm{HO} 2$ & $\mathrm{C} 2$ & $\mathrm{C}$ & & $\mathrm{C} 3$ & H3 \\
\hline BOND & $\mathrm{C} 3$ & 03 & & 03 & $\mathrm{HO} 3$ & C3 & $\mathrm{C} 4$ & $\mathrm{C} 4$ & $\mathrm{H}$ & & $\mathrm{C} 4$ & O4 \\
\hline BOND & O4 & $\mathrm{HO} 4$ & & $\mathrm{C} 4$ & C5 & $\mathrm{C} 5$ & H51 & $\mathrm{C} 5$ & $\mathrm{H}$ & 52 & & \\
\hline BOND & C5 & 05 & & & & & & & & & & \\
\hline DONO & BLNK & HOI & & & & & & & & & & \\
\hline DONO & BLNK & $\mathrm{HO} 2$ & & & & & & & & & & \\
\hline DONO & BLNK & $\mathrm{HO} 3$ & & & & & & & & & & \\
\hline DONO & BLNK & $\mathrm{HO} 4$ & & & & & & & & & & \\
\hline $\mathrm{ACCE}$ & $\mathrm{O} 1$ & & & & & & & & & & & \\
\hline $\mathrm{ACCE}$ & $\mathrm{O} 2$ & & & & & & & & & & & \\
\hline $\mathrm{ACCE}$ & 03 & & & & & & & & & & & \\
\hline $\mathrm{ACCE}$ & O4 & & & & & & & & & & & \\
\hline $\mathrm{ACCE}$ & 05 & & & & & & & & & & & \\
\hline$!$ & $I$ & $\mathrm{~J}$ & $\mathrm{~K}$ & $\mathrm{~L}$ & & $\mathrm{R}(\mathrm{IK})$ & $\mathrm{T}(\mathrm{IKJ})$ & PHI & & $\mathrm{T}(\mathrm{JKL})$ & & $\mathrm{R}(\mathrm{KL})$ \\
\hline IC & O1 & $\mathrm{C} 2$ & $* \mathrm{C} 1$ & $\mathrm{H} 1$ & & 1.4115 & 105.82 & -120.26 & & 110.81 & & 1.0905 \\
\hline IC & O1 & 05 & $* \mathrm{C} 1$ & $\mathrm{C} 2$ & & 1.4115 & 113.00 & -118.92 & & 111.32 & & 1.5218 \\
\hline IC & $\mathrm{O} 2$ & $\mathrm{C} 3$ & * $\mathrm{C} 2$ & $\mathrm{H} 2$ & & 1.4190 & 110.99 & $-116.9^{\prime}$ & & 108.77 & & 1.0892 \\
\hline
\end{tabular}




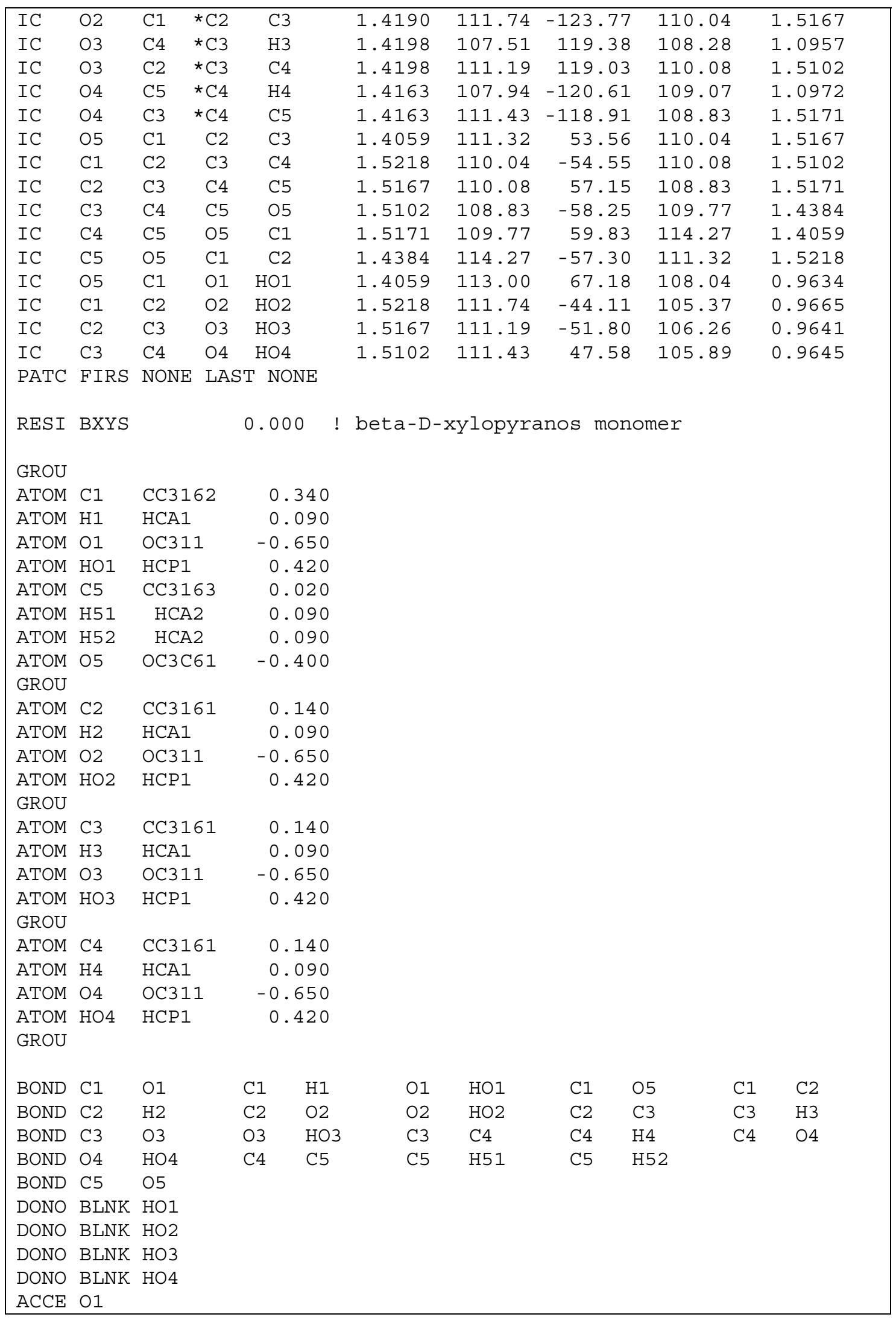




\begin{tabular}{|c|c|c|c|c|c|c|c|c|c|}
\hline ACCE & $\mathrm{O} 2$ & & & & & & & & \\
\hline $\mathrm{ACCE}$ & 03 & & & & & & & & \\
\hline $\mathrm{ACCE}$ & O4 & & & & & & & & \\
\hline $\mathrm{ACCE}$ & 05 & & & & & & & & \\
\hline$!$ & I & $\mathrm{J}$ & $\mathrm{K}$ & $\mathrm{L}$ & $R(I K)$ & $\mathrm{T}(\mathrm{IKJ})$ & PHI & $\mathrm{T}(\mathrm{JKL})$ & $\mathrm{R}(\mathrm{KL})$ \\
\hline IC & $\mathrm{O} 1$ & $\mathrm{C} 2$ & ${ }^{*} \mathrm{C} 1$ & H1 & 1.3890 & 105.75 & 114.54 & 108.17 & 1.0950 \\
\hline IC & $\mathrm{O} 1$ & 05 & ${ }^{*} \mathrm{C} 1$ & $\mathrm{C} 2$ & 1.3890 & 111.55 & 117.06 & 110.06 & 1.5340 \\
\hline IC & $\mathrm{O} 2$ & $\mathrm{C} 3$ & ${ }^{*} \mathrm{C} 2$ & $\mathrm{H} 2$ & 1.4154 & 112.27 & -118.21 & 108.23 & 1.0919 \\
\hline IC & $\mathrm{O} 2$ & $\mathrm{C} 1$ & ${ }^{*} \mathrm{C} 2$ & $\mathrm{C} 3$ & 1.4154 & 110.87 & -125.56 & 111.08 & 1.5253 \\
\hline IC & $\mathrm{O} 3$ & $\mathrm{C} 4$ & ${ }^{*} \mathrm{C} 3$ & H3 & 1.4157 & 110.61 & 120.65 & 108.81 & 1.1068 \\
\hline IC & 03 & $\mathrm{C} 2$ & ${ }^{*} \mathrm{C} 3$ & C4 & 1.4157 & 108.09 & 120.77 & 109.86 & 1.5177 \\
\hline IC & O4 & C5 & ${ }^{*} \mathrm{C} 4$ & $\mathrm{H} 4$ & 1.4252 & 110.90 & -120.61 & 108.35 & 1.1024 \\
\hline IC & O4 & $\mathrm{C} 3$ & ${ }^{*} \mathrm{C} 4$ & C5 & 1.4252 & 108.31 & -122.08 & 111.17 & 1.5287 \\
\hline IC & 05 & $\mathrm{C} 1$ & $\mathrm{C} 2$ & $\mathrm{C} 3$ & 1.4254 & 110.06 & 54.09 & 111.08 & 1.5253 \\
\hline IC & $\mathrm{C} 1$ & $\mathrm{C} 2$ & C3 & C4 & 1.5340 & 111.08 & -51.23 & 109.86 & 1.5177 \\
\hline IC & $\mathrm{C} 2$ & C3 & C4 & C5 & 1.5253 & 109.86 & 53.25 & 111.17 & 1.5288 \\
\hline IC & C3 & C4 & C5 & 05 & 1.5177 & 111.17 & -57.46 & 108.69 & 1.4274 \\
\hline IC & C4 & C5 & 05 & $\mathrm{C} 1$ & 1.5288 & 108.69 & 62.25 & 113.77 & 1.4254 \\
\hline IC & C5 & 05 & $\mathrm{C} 1$ & $\mathrm{C} 2$ & 1.4274 & 113.77 & -60.97 & 110.06 & 1.5340 \\
\hline IC & $\mathrm{C} 1$ & $\mathrm{C} 2$ & $\mathrm{O} 2$ & $\mathrm{HO} 2$ & 1.5340 & 110.87 & -100.51 & 112.13 & 0.9638 \\
\hline IC & $\mathrm{C} 2$ & $\mathrm{C} 3$ & $\mathrm{O} 3$ & $\mathrm{HO} 3$ & 1.5253 & 108.09 & -165.88 & 112.08 & 0.9730 \\
\hline IC & $\mathrm{C} 3$ & $\mathrm{C} 4$ & O4 & $\mathrm{HO} 4$ & 1.5177 & 108.31 & 134.18 & 106.97 & 0.9713 \\
\hline PATC & FIR & S NONE & $\mathrm{E}$ LA & ST NONE & & & & & \\
\hline RESI & BNAG & & & 0.000 & ! N-Acy & tyl-Gluc & cosamine & & \\
\hline \multicolumn{10}{|l|}{ GROU } \\
\hline ATOM & $\mathrm{C} 1$ & \multicolumn{2}{|c|}{ CC3162 } & 0.340 & & & & & \\
\hline ATOM & H1 & \multicolumn{2}{|c|}{ HCA1 } & 0.090 & & & & & \\
\hline ATOM & $\mathrm{O} 1$ & \multicolumn{2}{|l|}{ OC311 } & -0.650 & & & & & \\
\hline АTOM & $\mathrm{HOI}$ & \multicolumn{2}{|l|}{ HCP1 } & 0.420 & & & & & \\
\hline АTOM & C5 & \multicolumn{2}{|l|}{ CC 3163} & 0.110 & & & & & \\
\hline ATOM & H5 & \multicolumn{2}{|c|}{ HCA 1} & 0.090 & & & & & \\
\hline АTOM & 05 & & -0.400 & & & & & \\
\hline GROU & & & & & & & & & \\
\hline АTOM & $\mathrm{C} 2$ & \multicolumn{2}{|c|}{ CC3161 } & 0.070 & & & & & \\
\hline АTOM & $\mathrm{H} 2$ & \multicolumn{2}{|c|}{ HCA1 } & 0.090 & & & & & \\
\hline АTOM & NL & \multicolumn{2}{|l|}{ NH1 } & -0.47 & & & & & \\
\hline ATOM & $\mathrm{HL}$ & \multirow{2}{*}{\multicolumn{2}{|c|}{$\mathrm{H}$}} & 0.31 & & & & & \\
\hline GROUP & & & & & & & & & \\
\hline ATOM & CL & CT3 & \multicolumn{2}{|c|}{-0.27} & & & & & \\
\hline АTOM & HL1 & $\mathrm{HA}$ & \multicolumn{2}{|c|}{0.09} & & & & & \\
\hline ATOM & HL2 & $\mathrm{HA}$ & \multicolumn{2}{|c|}{0.09} & & & & & \\
\hline АTOM & HL3 & $\mathrm{HA}$ & \multirow{2}{*}{\multicolumn{2}{|c|}{0.09}} & & & & & \\
\hline \multicolumn{8}{|c|}{ GROUP } & & \\
\hline ATOM & CLP & C & \multicolumn{2}{|c|}{0.51} & & & & & \\
\hline ATOM & $\mathrm{OL}$ & 0 & & 51 & & & & & \\
\hline \multicolumn{10}{|c|}{ GROUP } \\
\hline GROU & & & & & & & & & \\
\hline АTOM & C3 & CC3161 & & 0.140 & & & & & \\
\hline ATOM & H3 & HCA1 & & 0.090 & & & & & \\
\hline ATOM & $\mathrm{O} 3$ & OC311 & & -0.650 & & & & & \\
\hline ATOM & $\mathrm{HO} 3$ & HCP1 & & 0.420 & & & & & \\
\hline
\end{tabular}




\begin{tabular}{|c|c|c|c|c|c|c|c|c|c|c|c|}
\hline ATOM & $\mathrm{C} 4$ & \multicolumn{2}{|c|}{ CC3161 } & 0.14 & & & & & & & \\
\hline ATOM & $\mathrm{H} 4$ & \multicolumn{2}{|c|}{ HCA1 } & 0.09 & & & & & & & \\
\hline ATOM & 04 & \multicolumn{2}{|c|}{ OC311 } & -0.65 & & & & & & & \\
\hline $\begin{array}{l}\text { ATOM } \\
\text { GROU }\end{array}$ & HO4 & \multicolumn{2}{|c|}{$\mathrm{HCP} 1$} & 0.42 & & & & & & & \\
\hline АTOM & $\mathrm{C} 6$ & \multicolumn{2}{|c|}{$\mathrm{CC} 321$} & 0.05 & & & & & & & \\
\hline АTOM & H61 & \multicolumn{2}{|l|}{ HCA2 } & 0.09 & & & & & & & \\
\hline ATOM & H62 & \multicolumn{2}{|l|}{ HCA2 } & 0.09 & & & & & & & \\
\hline ATOM & 06 & \multicolumn{2}{|c|}{ OC311 } & -0.65 & & & & & & & \\
\hline ATOM & HO6 & \multicolumn{2}{|c|}{$\mathrm{HCP} 1$} & 0.42 & & & & & & & \\
\hline BOND & $\mathrm{C} 1$ & $\mathrm{O} 1$ & $\mathrm{C} 1$ & $\mathrm{H} 1$ & & 01 & $\mathrm{HOI}$ & $\mathrm{C} 1$ & 05 & $\mathrm{C} 1$ & $\mathrm{C} 2$ \\
\hline BOND & $\mathrm{C} 2$ & $\mathrm{H} 2$ & $\mathrm{C} 2$ & $\mathrm{NL}$ & & NL & $\mathrm{HL}$ & $\mathrm{NL}$ & CLP & $\mathrm{C} 2$ & C3 \\
\hline BOND & C3 & 03 & $\mathrm{O} 3$ & Hо & 03 & $\mathrm{C} 3$ & $\mathrm{C} 4$ & $\mathrm{C} 4$ & $\mathrm{H} 4$ & $\mathrm{C} 4$ & O4 \\
\hline BOND & $\mathrm{O} 4$ & $\mathrm{HO} 4$ & $\mathrm{C} 4$ & $\mathrm{C} 5$ & & C5 & H5 & C5 & $\mathrm{C} 6$ & $\mathrm{C} 6$ & H61 \\
\hline BOND & $\mathrm{C} 6$ & H62 & $\mathrm{C} 6$ & 06 & & 06 & HO6 & C5 & \multirow{2}{*}{\multicolumn{2}{|c|}{05}} & H3 \\
\hline BOND & $\mathrm{CL}$ & HL1 & CL & $\mathrm{HL}$ & 52 & CL & HL3 & CL & $\mathrm{CL}$ & & \\
\hline \multicolumn{12}{|c|}{ DOUBLE CLP OL } \\
\hline IMPR & \multicolumn{11}{|c|}{ CLP CL NL OL } \\
\hline DONO & \multicolumn{11}{|c|}{ BLNK HOI } \\
\hline DONO & BLNK & $\mathrm{HO} 3$ & & & & & & & & & \\
\hline DONO & BLNK & $\mathrm{HO} 4$ & & & & & & & & & \\
\hline DONO & BLNK & HO6 & & & & & & & & & \\
\hline $\mathrm{ACCE}$ & 01 & & & & & & & & & & \\
\hline $\mathrm{ACCE}$ & 03 & & & & & & & & & & \\
\hline $\mathrm{ACCE}$ & 04 & & & & & & & & & & \\
\hline $\mathrm{ACCE}$ & 05 & & & & & & & & & & \\
\hline $\mathrm{ACCE}$ & 06 & & & & & & & & & & \\
\hline$!$ & I & $\mathrm{J}$ & $\mathrm{K}$ & $\mathrm{L}$ & & $R(I K)$ & $\mathrm{T}(\mathrm{IKJ})$ & PHI & & $\mathrm{T}(\mathrm{JKL})$ & $\mathrm{R}(\mathrm{KL})$ \\
\hline IC & 01 & $\mathrm{C} 2$ & ${ }^{*} \mathrm{C} 1$ & $\mathrm{H} 1$ & & 1.3890 & 105.75 & 114.5 & & 108.17 & 1.0950 \\
\hline IC & 01 & 05 & ${ }^{*} \mathrm{C} 1$ & $\mathrm{C} 2$ & & 1.3890 & 111.55 & 117.0 & & 110.06 & 1.5340 \\
\hline IC & 03 & $\mathrm{C} 4$ & *C3 & H3 & & 1.4157 & 110.61 & 120 & & 108.81 & 1.1068 \\
\hline IC & 03 & $\mathrm{C} 2$ & $* \mathrm{C} 3$ & $\mathrm{C} 4$ & & 1.4157 & 108.09 & 120. & & 109.86 & 1.5177 \\
\hline IC & 04 & $\mathrm{C} 5$ & * $\mathrm{C} 4$ & $\mathrm{H} 4$ & & 1.4252 & 110.90 & -120 & & 108.35 & 1.1024 \\
\hline IC & O4 & C3 & ${ }^{*} \mathrm{C} 4$ & C5 & & 1.4252 & 108.31 & -120 & & 111.17 & 1.5287 \\
\hline IC & C6 & 05 & *C5 & H5 & & 1.5099 & 108.10 & 118.6 & & 109.65 & 1.1042 \\
\hline IC & C6 & $\mathrm{C} 4$ & * $\mathrm{C} 5$ & 05 & & 1.5099 & 111.57 & 119.1 & & 108.69 & 1.4274 \\
\hline IC & 06 & H62 * & *C6 & H61 & & 1.4132 & 110.47 & -120.3 & & 107.85 & 1.0945 \\
\hline IC & 06 & C5 * & ${ }^{*} \mathrm{C} 6$ & H62 & & 1.4132 & 110.45 & -121.5 & & 108.99 & 1.0959 \\
\hline IC & 05 & $\mathrm{C} 1$ & $\mathrm{C} 2$ & $\mathrm{C} 3$ & & 1.4254 & 110.06 & 54.0 & & 111.08 & 1.5253 \\
\hline IC & $\mathrm{C} 1$ & $\mathrm{C} 2$ & C3 & C4 & & 1.5340 & 111.08 & -51.2 & & 109.86 & 1.5177 \\
\hline IC & $\mathrm{C} 2$ & $\mathrm{C} 3$ & C4 & C5 & & 1.5253 & 109.86 & 53.2 & & 111.17 & 1.5288 \\
\hline IC & C3 & C4 & C5 & 05 & & 1.5177 & 111.17 & -57.46 & & 108.69 & 1.4274 \\
\hline IC & C4 & C5 & 05 & $\mathrm{C} 1$ & & 1.5288 & 108.69 & 62.2 & & 113.77 & 1.4254 \\
\hline IC & C5 & 05 & $\mathrm{C} 1$ & $\mathrm{C} 2$ & & 1.4274 & 113.77 & $-60.9^{\prime}$ & & 110.06 & 1.5340 \\
\hline IC & $\mathrm{C} 4$ & C5 & C6 & 06 & & 1.5287 & 111.57 & 180. & & 110.45 & 1.4132 \\
\hline IC & 05 & $\mathrm{C} 1$ & $\mathrm{O} 1$ & $\mathrm{HO}$ & & 1.4254 & 111.55 & -60 . & & 107.83 & 0.9684 \\
\hline IC & $\mathrm{C} 1$ & $\mathrm{C} 2$ & $\mathrm{O} 2$ & $\mathrm{HO} 2$ & & 1.5340 & 110.87 & -60. & & 112.13 & 0.9638 \\
\hline IC & $\mathrm{C} 2$ & $\mathrm{C} 3$ & 03 & $\mathrm{HO} 3$ & & 1.5253 & 108.09 & 60 & & 112.08 & 0.9730 \\
\hline IC & $\mathrm{C} 3$ & $\mathrm{C} 4$ & O4 & $\mathrm{HO} 4$ & & 1.5177 & 108.31 & 60. & & 106.97 & 0.9713 \\
\hline IC & $\mathrm{C} 5$ & $\mathrm{C} 6$ & 06 & HOG & & 1.5099 & 110.44 & -60 & & 107.72 & 0.9641 \\
\hline ic $c 2$ & $2 \quad \mathrm{clp}$ & $0 * n 1$ & hl & 0.0 & 0.0 & 180.0 & 0.00 & .0 & & & \\
\hline ic $\mathrm{nl}$ & $\mathrm{Cl}$ & $*_{\mathrm{Clp}}$ & p ol & 0.0 & 0.0 & 180.0 & 0.00 & .0 & & & \\
\hline ic $\mathrm{Cl}$ & clp & $\mathrm{Onl}$ & $\mathrm{c} 2$ & 0.0 & 0.0 & 180.0 & 0.00 & .0 & & & \\
\hline ic ol & clp & $\mathrm{n} \mathrm{nl}$ & c2 & 0.0 & 0.0 & 0.0 & 0.00 & .0 & & & \\
\hline
\end{tabular}




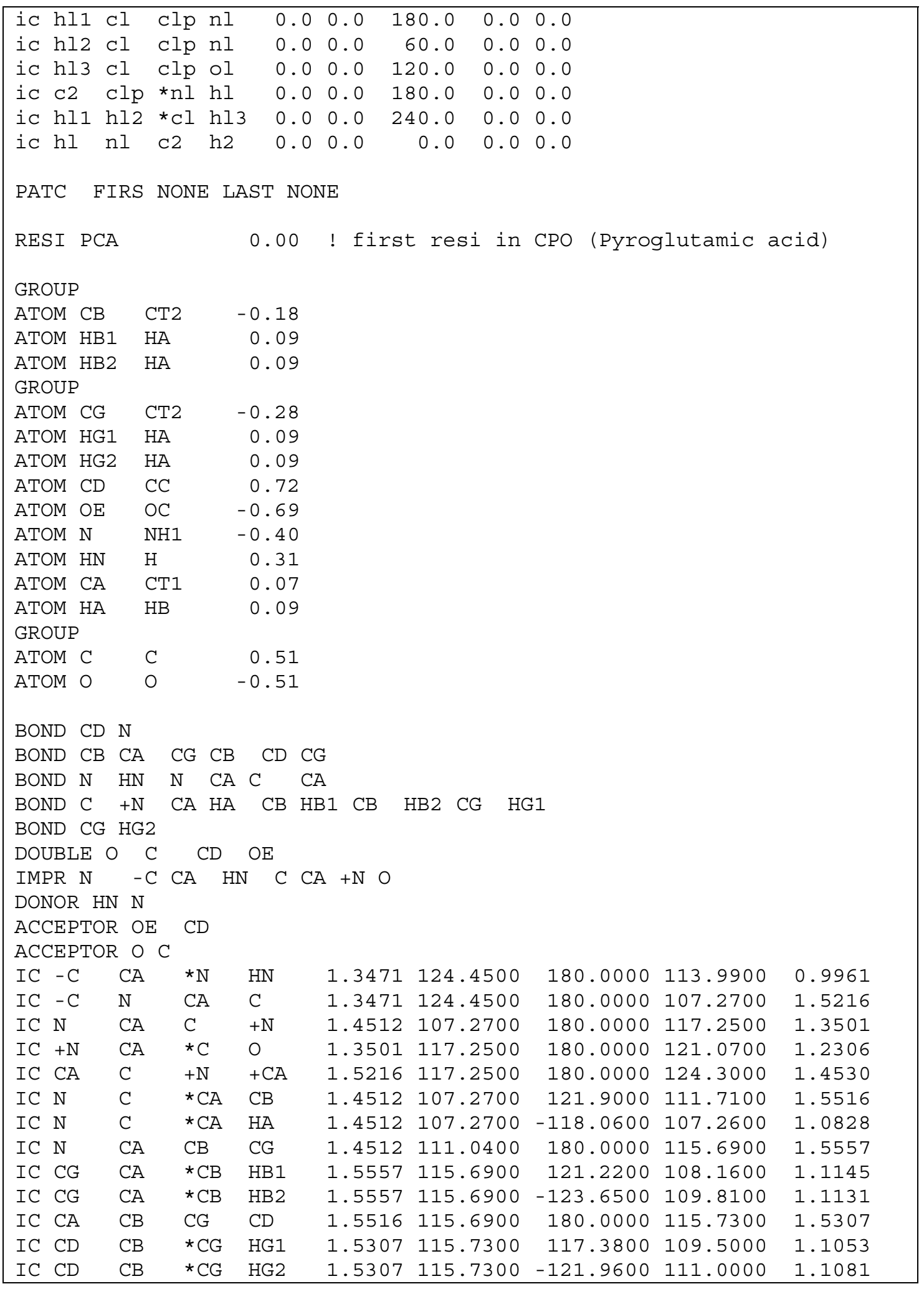

Table 50: Topology file for sugars, for n-acytyl-glucosamine and for pyroglutamic acid, which are found in CPO, and for which parameters were created by analogy 


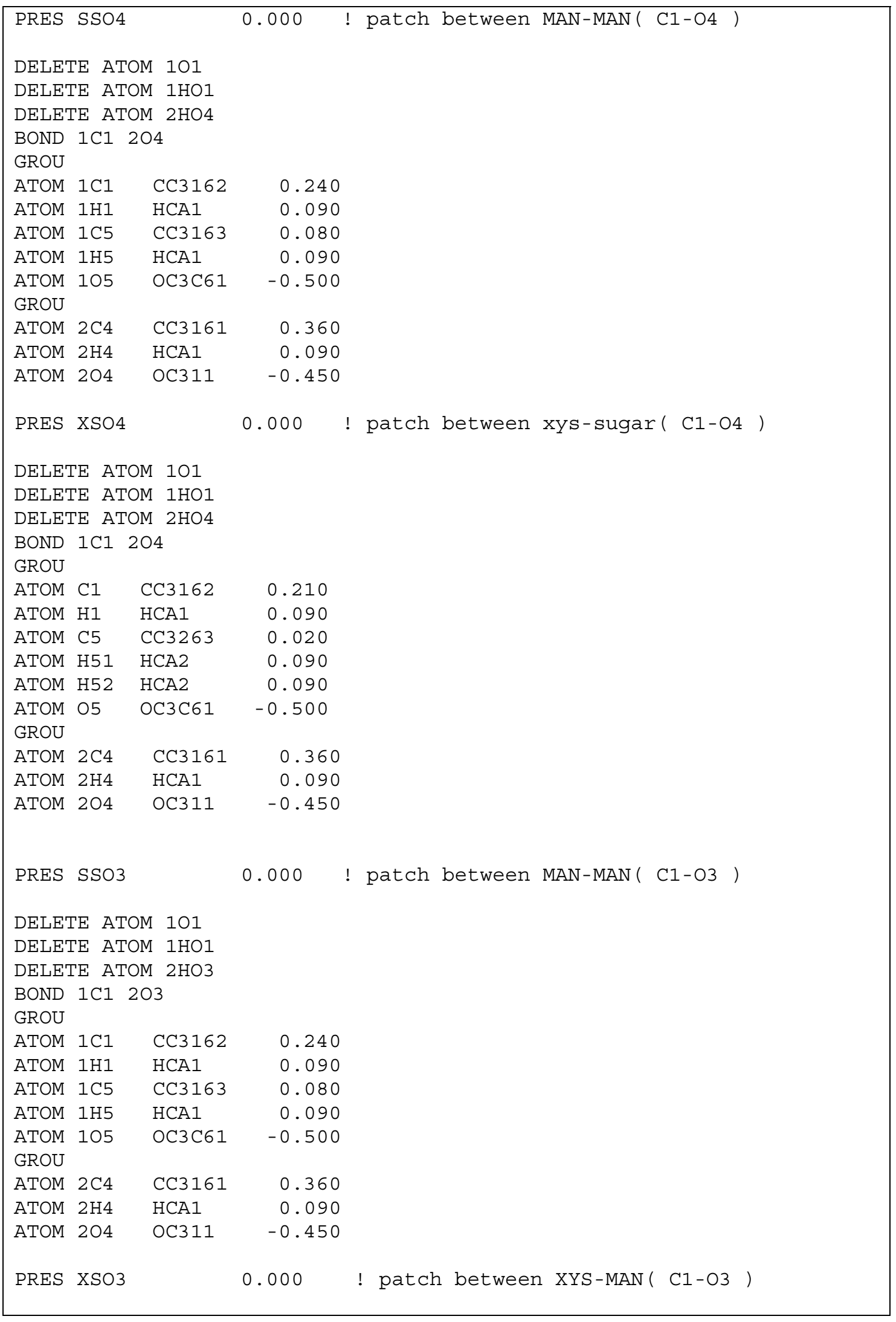




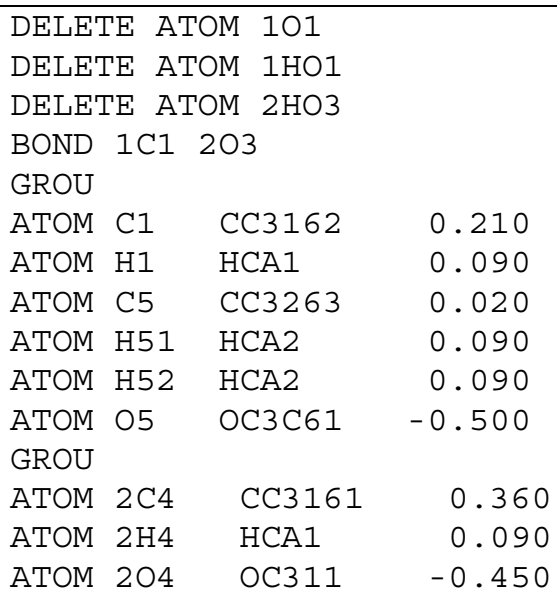




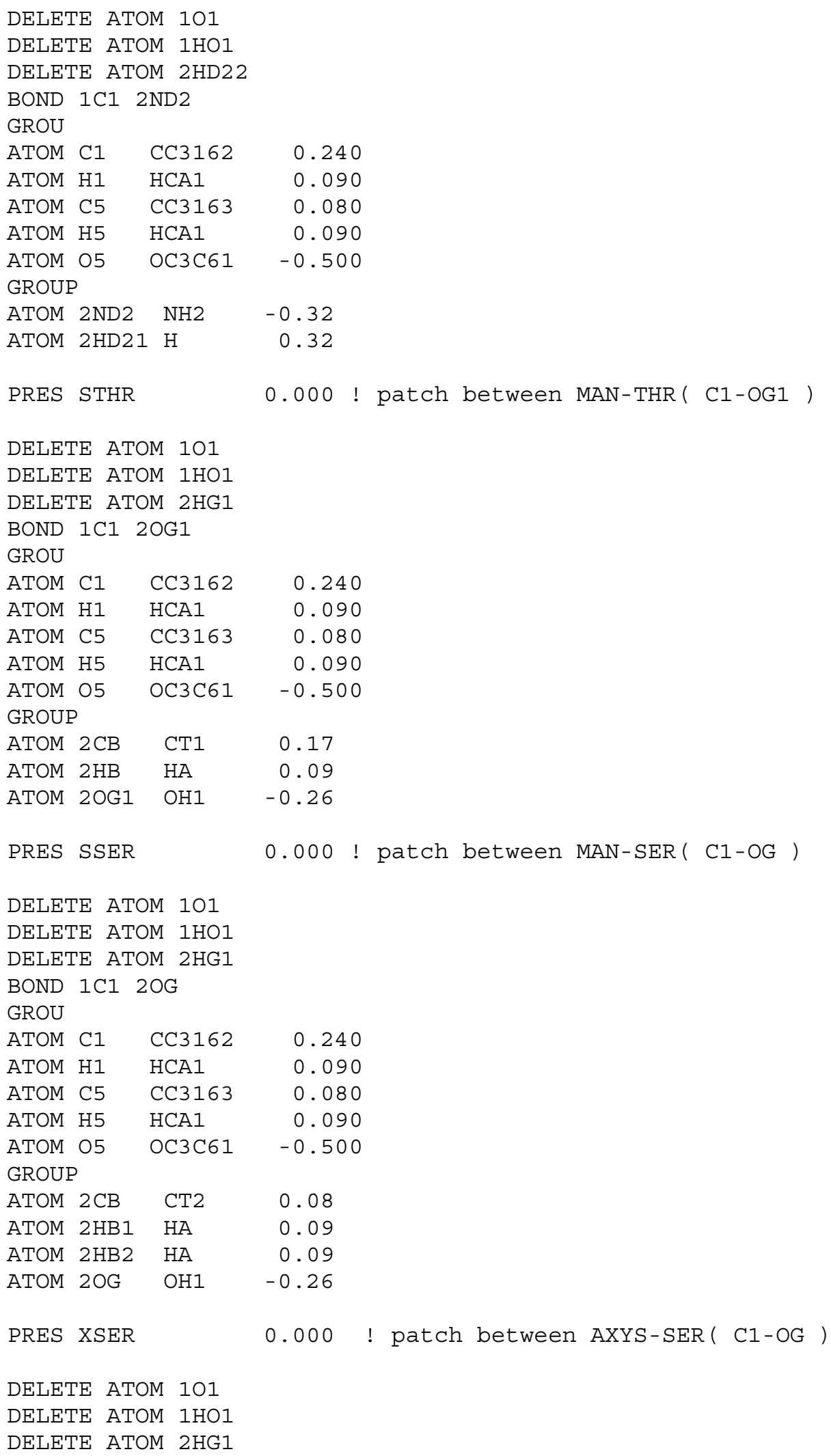




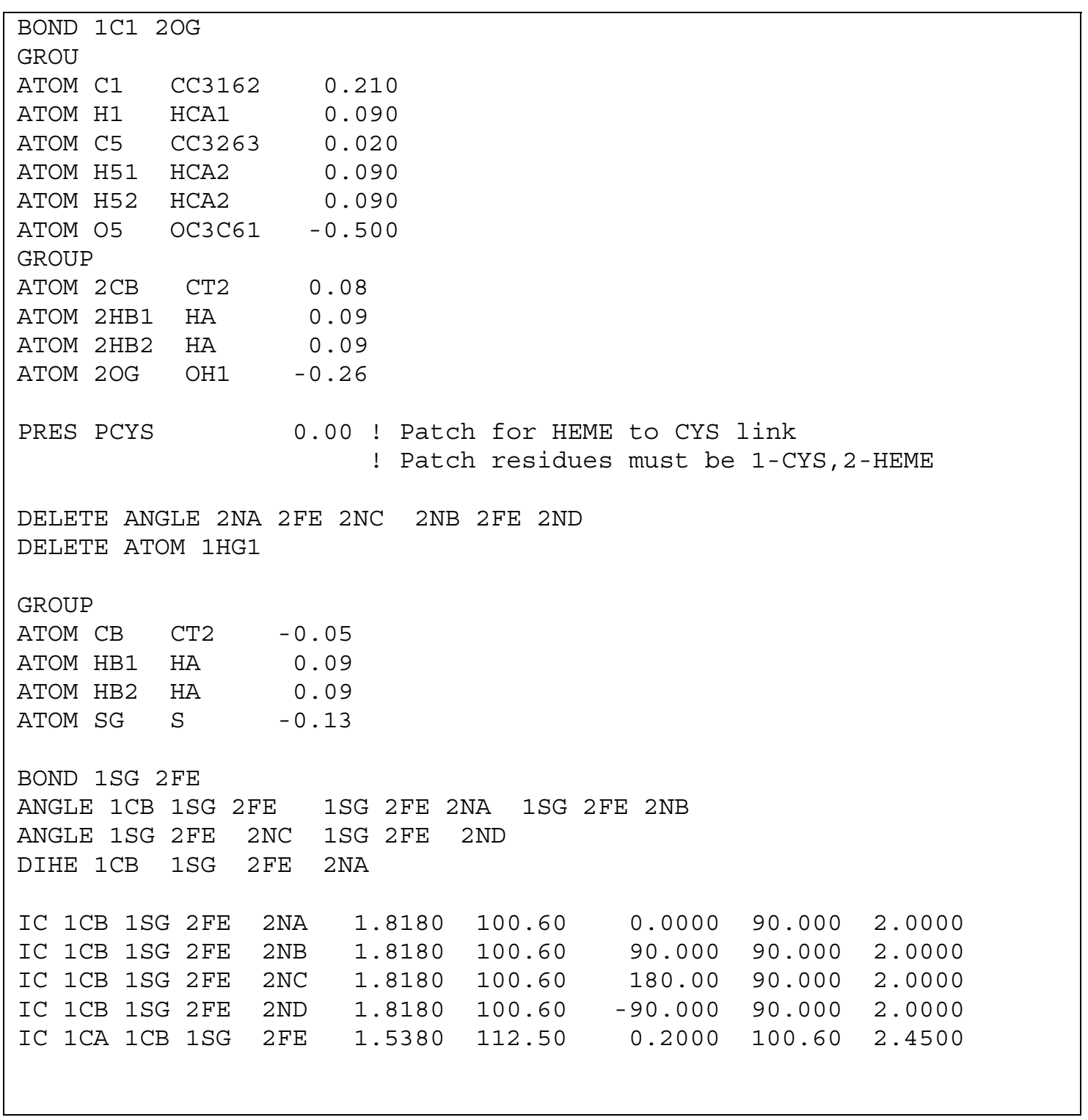

Table 51: Patches used to attach sugar-sugar molecules, sugar-amino acids and heme-cystine

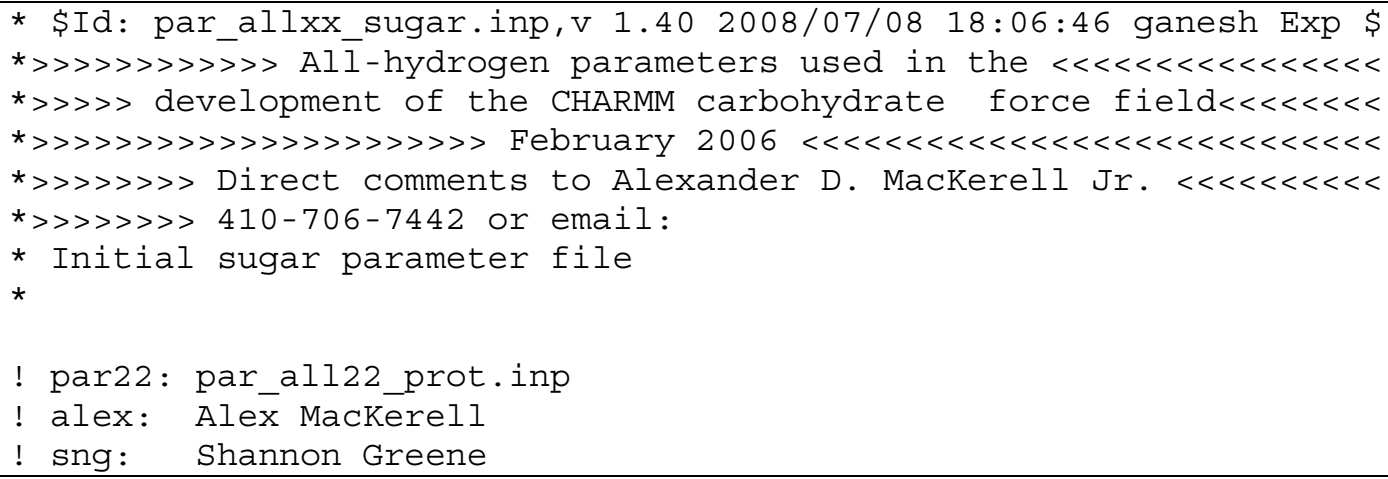




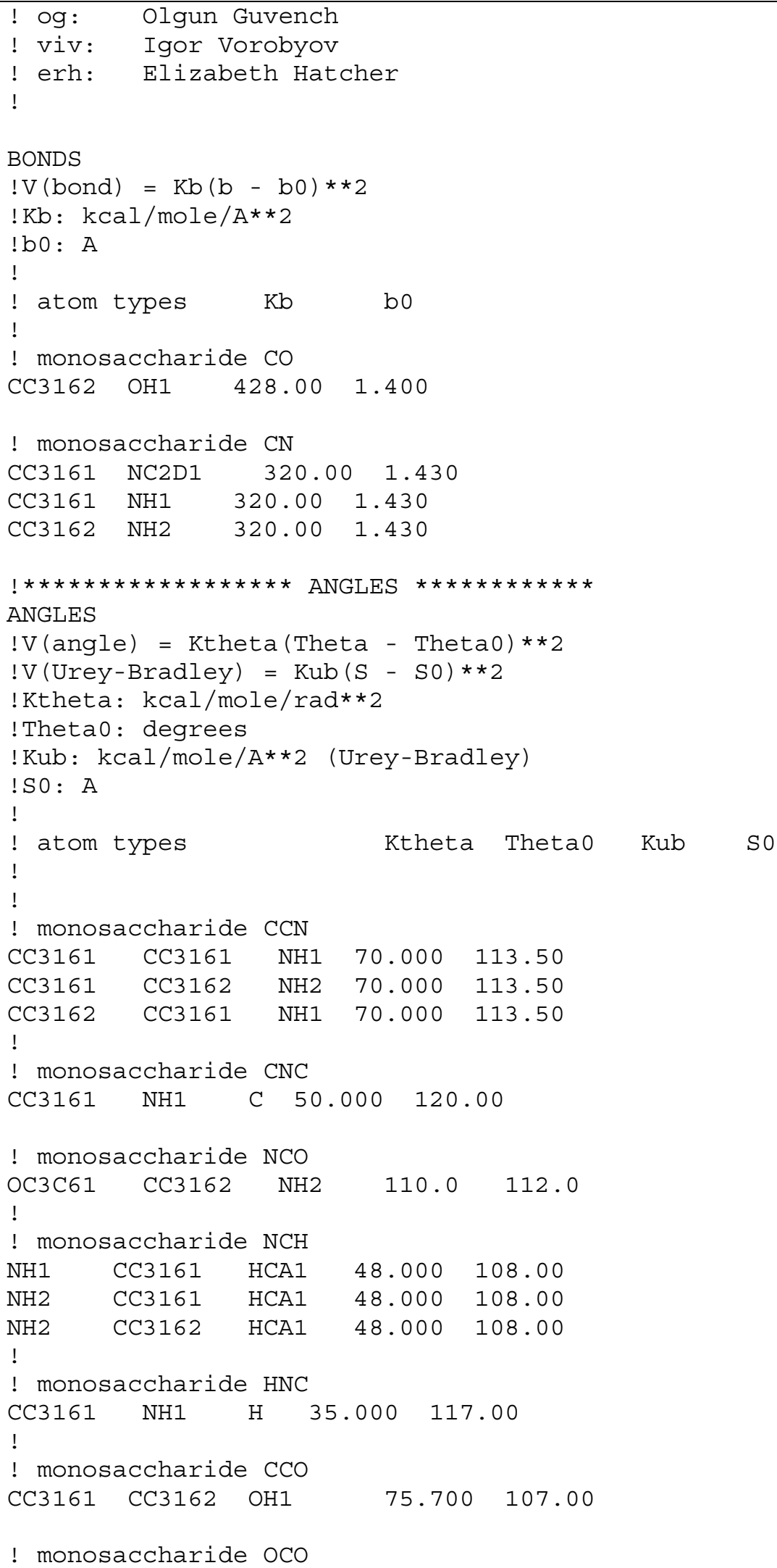




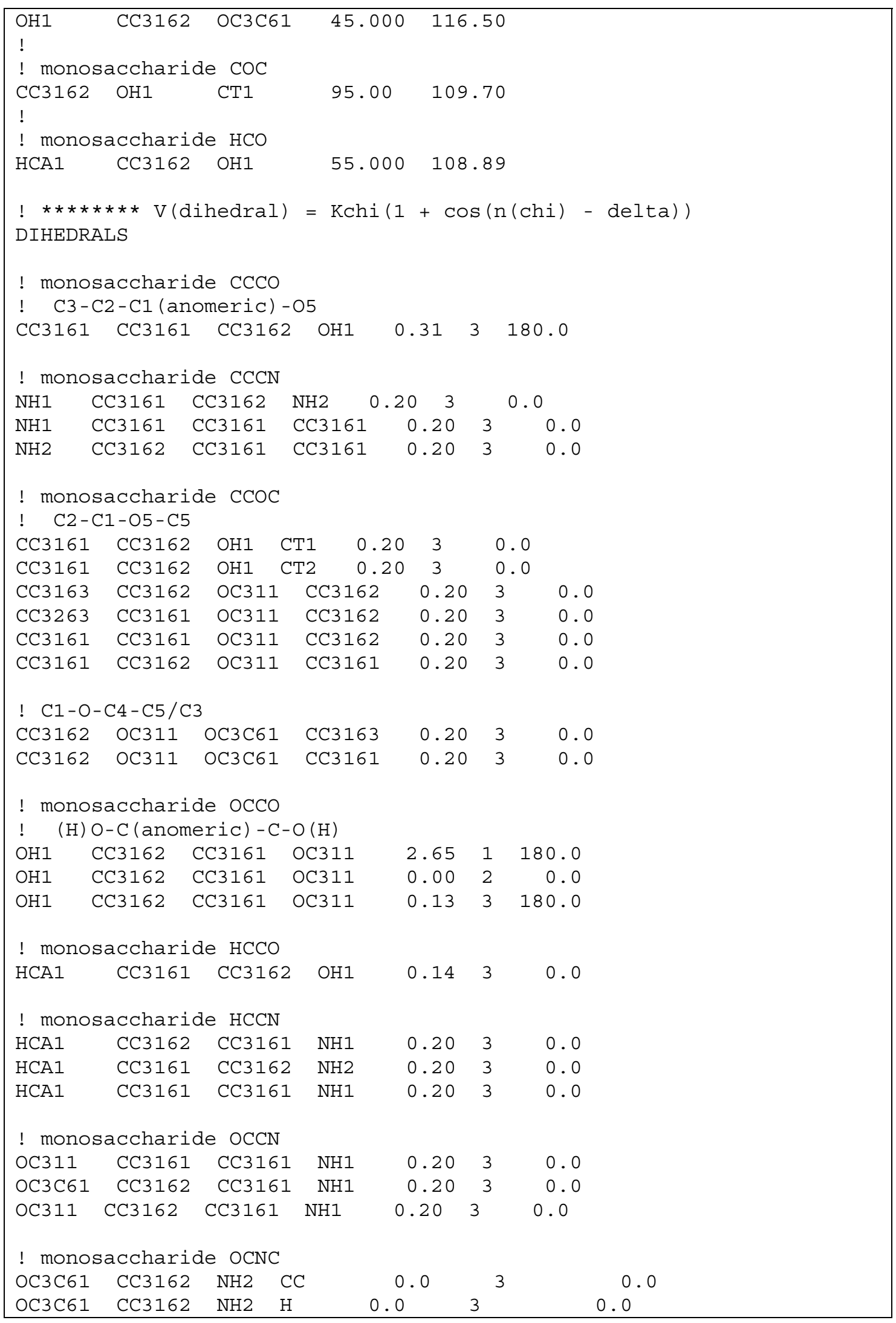




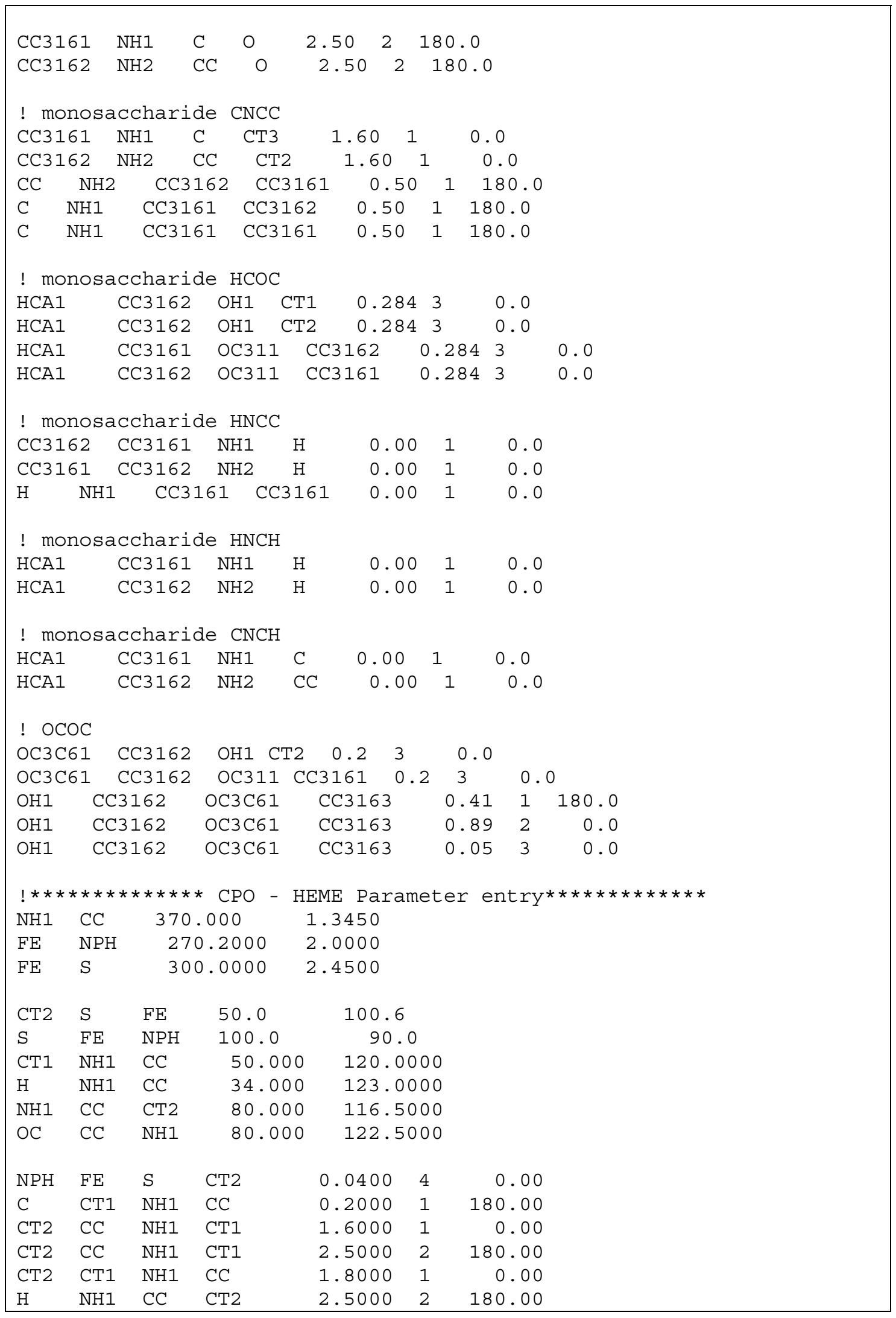




\begin{tabular}{|c|c|c|c|c|c|c|}
\hline $\mathrm{HB}$ & CT1 & $\mathrm{NH1}$ & $\mathrm{CC}$ & 0.0000 & 1 & 0.00 \\
\hline $\mathrm{OC}$ & $\mathrm{CC}$ & $\mathrm{NH} 1$ & $\mathrm{CT} 1$ & 2.5000 & 2 & 180.00 \\
\hline $\mathrm{OC}$ & $\mathrm{CC}$ & $\mathrm{NHI}$ & $\mathrm{H}$ & 2.5000 & 2 & 180.00 \\
\hline
\end{tabular}

Table 52: Lines appended by Cassian to CHARMM parameter file in order to include parameters developed for entries in topology file in Appendix B1 
VITA

\title{
CASSIAN D’CUNHA
}

\author{
$1995-1999$ \\ Bachelor of Engineering: Chemical Engineering \\ University of Mumbai \\ Mumbai, India \\ $2000-2003$ \\ Master of Science: Computer Science \\ Florida International University \\ Miami, Florida, USA \\ 2004 \\ Cyberbridges Scholarship \\ Florida International University \\ Miami, Florida, USA \\ $2005-2010$ \\ Teaching Assistant \\ Florida International University \\ Miami, Florida, USA \\ 2011 \\ Guest lecturer: Physical Chemistry II \\ Florida International University \\ Miami, Florida, USA \\ Dissertation Year Fellowship \\ Florida International University \\ Miami, Florida, USA
}

\section{PUBLICATIONS AND PRESENTATIONS}

D. C. Chatfield, A. Augsten, C. D'Cunha, and S. E. Wong, "Methyl dynamics in

crystalline amino acids: MD and NMR,” J. Comput. Chem. 24, 1052-1058, 2003.

C. D’Cunha, D. C. Chatfield, A. Augsten, "Correlation times and adiabatic barriers for methyl rotations in proteins," FAME, Orlando, Summer 2004.

D. C. Chatfield, A. Augsten, and C. D'Cunha, "Correlation times and adiabatic barriers for methyl rotations in proteins," J. Biomol. NMR. 1-9, 2004.

C. D'Cunha, A. Gairola, A. Augsten, and D. C. Chatfield, "MD/NMR Characterization of internal motions of peptide toxins," American Chemical Society meeting, Philadelphia, Fall 2004. 
D. C. Chatfield, A. Augsten, C. D'Cunha, E. Lewandowska, and S. F. Wnuk, "Theoretical and Experimental Study of Regioselectivity of Michael Additions," Eur. J. Org. Chem. 313-322, 2004.

C. D’Cunha, D. Chatfield, H. L. Alvarez, D. A. Cox, E. Crumpler, R. Gutierrez, J. Ibarra, E. Johnson, K. Kumar, T. Milledge, G. Narasimhan, S. M. Sadjadi, C. Zhang, "CyberBridges A Model Collaboration Infrastructure for e-Science," SuperComputing 2006, Tampa, Fall 2006 (oral presentation).

H. L. Alvarez, D. Chatfield, D. A. Cox, E. Crumpler, C. D’Cunha, R. Gutierrez, J. Ibarra, E. Johnson, K. Kumar, T. Milledge, G. Narasimhan, S. M. Sadjadi, C. Zhang, "CyberBridges A Model Collaboration Infrastructure for e-Science," Proceedings of the 7th IEEE International Symposium on Cluster Computing and the Grid (CCGrid'07), Rio de Janeiro, Brazil, May 2007.

C. D'Cunha and D. C. Chatfield, "Study of the halogenation mechanism catalyzed by Chloroperoxidase," American Chemical Society meeting, Philadelphia, Fall 2008.

C. D'Cunha and D. C. Chatfield, "Study of the halogenation mechanism catalyzed by Chloroperoxidase," 3rd CMM 'Users Meet Developers' Workshop on QM/MM Simulations, Philadelphia, Fall 2008.

A. N. Morozov, C. D'Cunha, C. A. Alvarez, and D. C. Chatfield "Enantiospecificity of Chloroperoxidase-Catalyzed Epoxidation: Biased Molecular Dynamics Study of a Cis- $\beta$ Methylstyrene/Chloroperoxidase-Compound I Complex," Biophys. J. 100(4), 1066-1075, 2011. 